\title{
THE ROLE OF ADIPONECTIN IN REGULATION OF METABOLISM IN DAIRY COWS
}

\author{
By \\ Konstantinos D. Kalamaras Bsc, Msc \\ Thesis submitted to the University of Nottingham for the \\ Degree of Doctor of Philosophy
}

2012

The University of

Nottingham

\author{
DIVISION OF ANIMAL SCIENCES \\ SCHOOL OF BIOSCIENCES \\ SUTTON BONINGTON CAMPUS \\ UNITED KINGDOM
}


The real constitution of things is accustomed to hide itself........

Everything flows.......

Heraclitus 540 BCE-480 BCE

Greek philosopher 
It is dedicated to my father, Demetrios, and my mother, Agori. 


\section{DECLARATION}

I hereby declare that this thesis is my own work and that it has not been submitted anywhere for any other degree or award. The work presented herein is my own work and where other sources of information have been used, they have been acknowledged. I also declare that this thesis is less than 100,000 words in length, inclusive of tables, figures, appendices, footnotes, and references.

Konstantinos D. Kalamaras 


\begin{abstract}
Body condition score has been considered an indirect measure of nutritional status and nutrition has been demonstrated to interact with reproduction. Adiponectin and leptin, because of its insulin sensitising actions and their association with body condition could be potential regulators of metabolism during the transition from pregnancy to lactation. The work described in this $\mathrm{P} h \mathrm{D}$ project was designed to investigate the role of metabolic, body condition, and dietary factors in regulation of productive and reproductive performance in dairy cows, with particular regard to glucose homeostasis and adipokines. A special focus was directed to circulating adiponectin and its association with metabolic and hormonal status, and reproduction. Interestingly, adiponectin was found to be present in bovine milk at concentrations similar those of plasma. Body condition score, GH, insulin, leptin, and adiponectin showed a potential to modulate glucose homeostasis and reproductive output. Results from this study demonstrated a negative effect of long-term moderately elevated insulin concentration on reproductive performance. Additionally, BCS at calving and $\triangle \mathrm{BCS}$ are determinant factors influencing postpartum reproductive recovery and they should be basic components of farming monitoring schemes. Although further investigation is needed to address the proposed negative relationship between adiponectin and $\mathrm{GH}$, and to identify other dietary factors that may modulate circulating adiponectin, data from this study support regulatory roles of adiponectin in metabolism and reproduction. Moreover, adiponectin holds great promise to serve as a putative target molecule that integrates metabolism, reproduction and feeding behaviour. More importantly, hypoadiponectinemia could be another alternative mechanism that contributes to poor fertility in dairy cows. The incorporation of this new knowledge into the development of better nutritional strategies is a key point that is being considered to improve the welfare, and reproductive and productive performance in dairy cows.
\end{abstract}




\section{ACKNOWLEDGMENTS}

I am grateful to IKY (Greek State Scholarships Foundation) for the financial support provided for this project. Without IKY financial support, this work would not have been accomplished.

The main animal experiment, which provided data and blood samples for use in this study, was supported by Project LK0646 in the LINK Sustainable Livestock Production programme, which was funded by SEERAD, ABNA Ltd, BOCM PAULS Ltd and Provimi Ltd. Further support for animal work was provided by Defra grants LS3306 and LS3660.

I owe immense gratitude to my adviser and supervisor Professor Phil Garnsworthy. Throughout my degree, I have received from him generous support, constructive criticism, and invaluable guidance. His insights regarding statistical and practical matters in animal science have encouraged me to take the next step to becoming scientist. I have been very privileged to have him as supervisor. He has been not only enthusiastic, patient, friendly and helpful with me, but also impartial. Without his supervision, this work would have been unfulfilling.

I would like to express my deepest gratitude to Professor Bob Webb, my second supervisor, and Professor Julian Wiseman, my assessor, for providing valuable comments.

I would like to thank the following people for technical assistance: Neil Saunders, Marcus Mitchell, and Morag Hunter. Your empathy, support and patience were invaluable to help me understand laboratory techniques.

I also thank all those who helped me complete my Doctor of Philosophy degree in animal sciences. Many people have inspired me throughout this project, not least of which are those who have kept me sane, balanced and healthy. I would like to extend my cordial 
gratitude to my fellow PhD students: Kunal M. Saini (Plant science), Piotr Jasionowicz (Plant science), Nataly Nareidoo (Plant science) and Oliveira Caetano de Freitas Neto (Veterinary science and medicine). Thanks for the laughs and the support, it helped me out in some very tough personal times. I will miss you! I feel privileged to known you. I would also like to express my appreciation to all the people from Greece (Rosa Kuvelioti, Vlasis Gavriilides, and Apostolos Panteloglou) I have met in Sutton Bonington during my PhD programme, and were willing to share their time and friendship.

I am short of words to express my deepest gratitude and feelings to my parents (Demetrios and Agori), my brother (Sotirios), my sister (Euaggelia), my nephew (Nikos), and my brother-in-law (Giannis) for their unceasing support, love, and motivation. The inseparable support and courage they provided allowed me to always keep my spirits high and to keep my goals in sight. This work is dedicated to my parents who have always guided me emotionally and intellectually.

Last of all I would like to thank my fiancée Eleni (Elli) Vloumidi (PhD in Veterinary science and medicine). I am not sure how I would have gotten through the last few years without you. You are my love and my inspiration and I thank God I met you in Sutton Bonington. Your unwavering support, unconditional love, and encouragement made this work possible. 


\section{TABLE OF CONTENTS}

ABSTRACT
ACKNOWLEDGMENTS
ABBREVIATIONS
LIST OF TABLES
LIST OF FIGURES

1. INTRODUCTION \& PURPOSE OF THE STUDY ………………………………........

2. REVIEW OF LITERATURE...........................................................................

2.1. Postpartum nutritional strategies........................................................................ 5

2.2. Body condition score and body condition score management in dairy cows............ $\quad 7$

2.3. Glucose homeostasis and homeorhesis................................................................. 12

2.4. Pancreatic hormones and adipokines................................................................ 17

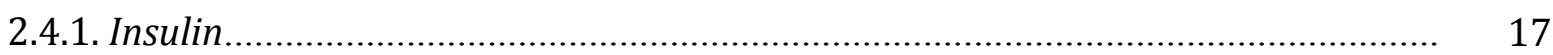

2.4.1.1. Insulin receptor and glucose transporters....................................................... 17

2.4.1.2. Effects of insulin on glucose metabolism and body reserves.............................. 19

2.4.1.3. Effects of insulin on milk yield and milk composition.......................................... 23

2.4.1.4. Insulin and GH/IGF-I system........................................................................ 25

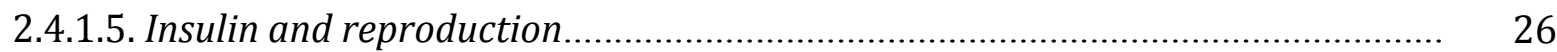

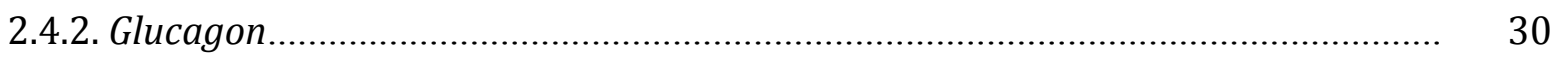

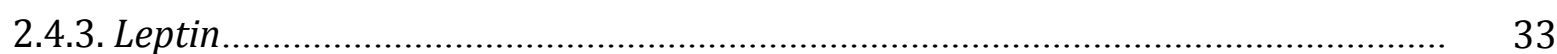

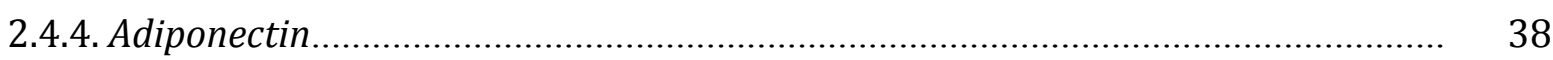

2.5. Working hypotheses and objectives..................................................................... 46

3. EFFECTS OF BODY CONDITION SCORE AT CALVING AND DIET ON CIRCULATING METABOLIC HORMONES, METABOLITES, AND REPRODUCTIVE TRAITS IN DAIRY COWS.

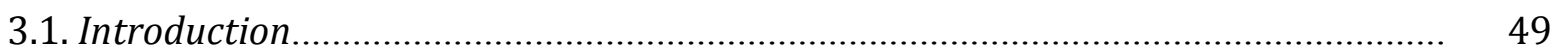

3.2. Materials \& methods...................................................................................

3.2.1. Data

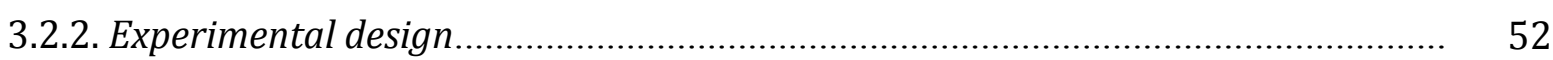

3.2.3. Feeding and milking....................................................................................... 53

3.2.4. Reproductive management............................................................................... 53

3.2.5. Recording, sampling and analysis.............................................................. 53

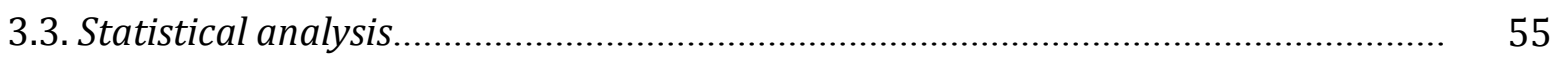

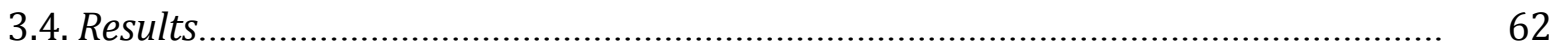

3.4.1. Consistency of BCS at calving tretment............................................................... 62

3.4.2. Effect of milk progesterone profile on pregnancy rate......................................... 62 
3.4.3. Effects of BCS at calving and diet on reproductive performance.

3.4.4. Effects of BCS at calving and diet on circulating metabolic hormones................... 63

3.4.5. Effects of BCS at calving and diet on circulating metabolites................................. 63

3.4.6. Effects of BCS at calving and diet on production traits.......................................... 63

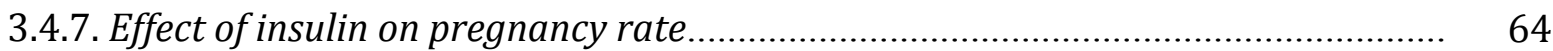

3.4.8. Effects of insulin, IGF-I, glucose, and parity on milk progesterone profile............. $\quad 65$

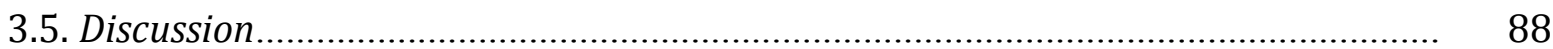

3.5.1. Effect of BCS at calving on circulating metabolic hormones, metabolites, and 88 reproductive traits in dairy cows.

3.5.2. Effect of diet on circulating metabolic hormones, metabolites, and reproductive traits in dairy cows.

3.5.3 Reproductive performance and optimum insulin concentration............................ 96

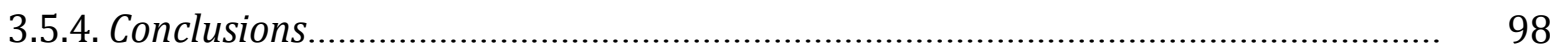

4. MEASUREMENT OF PLASMA AND MILK ADIPONECTIN, AND THE IMPACT OF LACTATION STAGE, DIET, AND BODY CONDITION AT CALVING ON PLASMA ADIPONECTIN CONCENTRATIONS IN DAIRY COWS.

4.1. Introduction.

4.2. Materials \& methods.

4.2.1. Measurement of adiponectin in bovine milk (Experiment 1).

4.2.2. Measurement of circulating adiponectin (Experiment 2).

4.2.2.1. Data.

4.2.2.2. Experimental design

4.2.2.3. Feeding and milking

4.2.2.4. Recording, sampling and analysis.

4.3. Statistical analysis.

103

4.3.1. Adiponectin in bovine milk (Experiment 1).

4.3.2. Circulating adiponectin (Experiment 2).

4.4. Results.

4.4.1. Adiponectin in bovine milk (Experiment 1)........................................................ 110

4.4.2. Circulating adiponectin (Experiment 2) .......................................................... 110

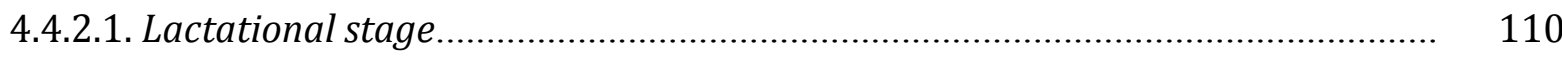

4.4.2.2. Diet and BCS at calving ................................................................................... 112

4.4.2.3. Associations of circulating adiponectin with circulating metabolites, 112

metabolic hormones, and production traits.

4.4.2.4. Relationships between DMI and circulating adiponectin, glucose, leptin,

MEBAL, and milk yield in FAT and THIN cows at calving..

4.5. Discussion

4.5.1. Measurement of plasma and milk adiponectin.

4.5.2. The impact of lactational stage on circulating adiponectin. 
4.5.3. The impact of diet and BCS at calving on circulating adiponectin.

\section{INTERRELATIONSHIPOF ADIPONECTIN WITH GLUCOSE HOMEOSTASIS AND THE EFFECT OF CIRCULATING ADIPONECTIN LEVELS ON REPRODUCTIVE PERFORMANCE IN DAIRY COWS}

5.1. Introduction

5.2. Materials \& methods

5.2.1. Data.

5.2.2. Experimental design

5.2.3. Feeding and milking.

5.2.5. Reproductive management.

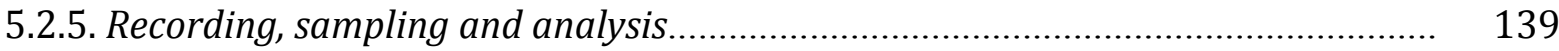

5.3. Statistical analysis

5.3.1. Effect of adiponectin levels on metabolic and hormonal profile, and reproductive performance.

5.3.2. Exploration of adiponectin interrelationships with glucose, BCS, metabolic hormones, and metabolites: a multivariate approach

5.4. Results.

5.4.1 The effect of circulating adiponectin levels on metabolic and hormonal profile, productive traits, and reproduction

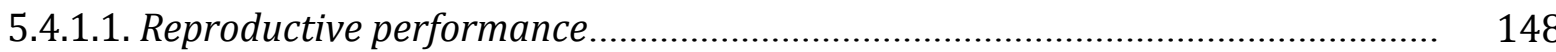

5.4.1.2. Metabolic profile, hormonal profile, and productive traits.

150

5.4.2 Adiponectin interrelationships with glucose, BCS, metabolic hormones, and metabolites.

5.5. Discussion

5.5.1. Effect of circulating adiponectin levels on metabolic hormones, metabolites, productive traits, and reproductive performance.

5.5.2. Interrelationships of adiponectin with glucose, BCS, metabolic hormones, and metabolites in high and low yielding cows.

\section{GENERAL DISCUSSION}

6.1. Introduction

6.2. Summary of main points.

6.2.1. Effect of diet and body condition score at calving on reproductive performance in dairy cows.

6.2.2. Reproductive performance and optimum insulin concentration.............................. 186

6.2.3. Adiponectin.

6.2.3.1. Measurement of circulating adiponectin in dairy cows.................................... 187

6.2.3.2. Measurement of adiponectin in bovine milk. 
6.2.3.3. The impact of lactational stage, diet and BCS at calving on circulating adiponectin in dairy cows.

6.2.3.4. Circulating adiponectin and milk yield.

6.2.3.5. Adiponectin and feed intake in dairy cows.

6.2.3.6. Effect of circulating adiponectin levels on reproductive performance.

6.2.4. Glucose homeostasis.

6.2.5. Reproductive performance and adipokines......................................................... 193

6.3. Integration of results and impact on reproductive management............................ 193

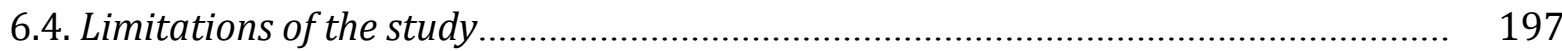

6.5. Recommendations for further research................................................................... 199

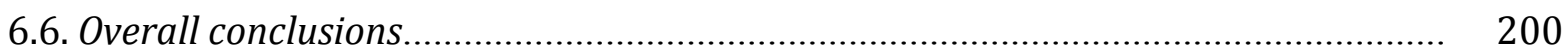

7. APPENDIX

A.1. Blood sampling and post-collection processing …................................................. 201

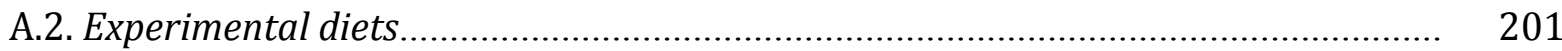

A.3. Measurement of adiponectin in blood plasma...................................................... 203

A.4. Measurement of adiponectin in milk ...................................................................... 208

A.5. Measurement of milk progesterone ..................................................................... 210

A.6. Measurement of metabolites in blood plasma......................................................... $\quad 212$

B.1. Parameter estimates of models were used to explore interrelationships of 213 adiponectin with glucose, BCS, metabolic hormones, and metabolites. 


\section{ABBREVIATIONS}

\begin{tabular}{ll}
\hline AA & Amino acids \\
AdipoR1 & Adiponectin receptor type I \\
AdipoR2 & Adiponectin receptor type II \\
ADP & Adiponectin \\
AIC & Akaike's information criterion \\
AMP & Adenosine monophosphate \\
AMPK & 5' AMP-activated protein kinase \\
AMY & Aggregated (cumulative) milk yield \\
ATP & Adenosine triphosphate \\
AUC Roc & Area under the ROC curve \\
BCS & Body condition score \\
BMI & Body mass index \\
BOHB & $\beta$-Hydroxybutyrate \\
bST & Bovine somatotropin \\
CAMP & Cyclic adenosine monophosphate \\
CFI & Comparative fitness index \\
CI & Confidence interval 95\% \\
CL & Corpus luteum \\
CLA & Commencement of luteal activity \\
CoA & Coenzyme A \\
CP & Crude protein \\
CV & Coefficient of variation \\
DIC & Deviance information criterion \\
DM & Dry matter \\
DMI & Dry matter intake \\
DOV1 & Delayed ovulation type I \\
DOV2 & Delayed ovulation type II \\
EB & Energy balance \\
ED & Effective dose of 80\% \\
EGF & Epidermal growth factors \\
ELISA & Enzyme-linked immunosorbant assay \\
ERK & Extracellular signal-regulated kinases \\
Exp $(\boldsymbol{b})$ & Exponentiated beta coefficient \\
FAO & Fatty acid oxidation \\
FFA & Free fatty acids \\
FSH & Follicle-stimulating hormone \\
GC & Glucocorticoids \\
&
\end{tabular}




\begin{tabular}{|c|c|}
\hline GDM & Gestational diabetes mellitus \\
\hline GEE & Generalized estimating equations \\
\hline GH & Growth hormone \\
\hline GHR & Growth hormone receptors \\
\hline GHRH & Growth hormone releasing hormone \\
\hline GLM & Generalized linear models \\
\hline GLUGON & Glucagon \\
\hline GLUT & Glucose transporter \\
\hline GnRH & Gonadotropin releasing hormone \\
\hline HF & High fat diet \\
\hline HPO & Hypothalamic pituitary ovary axis \\
\hline HS & High starch diet \\
\hline HSL & Hormone sensitive lipase \\
\hline ICV & Intra-cerebro-ventricular \\
\hline IGF-I & Insulin-Like Growth Factor I \\
\hline INS & Insulin \\
\hline IRS & Insulin receptor substrates \\
\hline LH & Luteinizing hormone \\
\hline LPL & Lipoprotein lipase \\
\hline LPN & Leptin \\
\hline LSD & Least square differences \\
\hline LWT & Live weight \\
\hline MANOVA & Multivariate analysis of variance \\
\hline MAPK & Mitogen-activated protein kinase \\
\hline MAR & Missing at random \\
\hline ME & Metabolizable energy \\
\hline MEBAL & Metabolizable energy balance \\
\hline MI & Multiple imputation \\
\hline ML & Maximum likelihood \\
\hline mRNA & Messenger ribonucleic acid \\
\hline MY & Milk yield \\
\hline$n-3$ PUFA & Omega- 3 polyunsaturated fatty acids \\
\hline NEB & Negative energy balance \\
\hline NEFA & Non-esterified fatty acids \\
\hline NFI & Normed fit index \\
\hline NPY & Neuropeptide Y \\
\hline
\end{tabular}




\begin{tabular}{ll} 
NS & Non-significant \\
Ob-R & Short form receptors of leptin \\
Ob-Rb & Long form receptor of leptin \\
OM & Organic matter \\
OR & Odds ratio \\
P4 & Progesterone \\
PCL1 & Persistent corpus luteum type I \\
PCL2 & Persistent corpus luteum type II \\
PCOS & Polycystic ovary syndrome \\
PDA & Path diagrams analysis \\
PMY & Previous milk yield \\
POMC & Pro-opiomelanocortin \\
PPA & Postpartum anoestrus \\
PPAR- $\alpha$ & Peroxisome proliferator-activated receptor alpha \\
QIC & Quasi-likelihood under the independence model criterion \\
REML & Restricted maximum likelihood \\
RIA & Radio immuno assay \\
RMSEA & Root-mean-square error of approximation \\
ROC & Receiver operating characteristic \\
SE & Standard error of means \\
SED & Standard error of the difference between treatment means \\
SEM & Structural equation modeling \\
TAG & Triacylglycerols \\
TG & Triglycerides \\
TGF & Transforming growth factors \\
TNF- $\alpha$ & Thiazor necrosis factor-alpha \\
TZD & Chi-square \\
VFA & \\
WAT & \\
$\chi^{2}$ & \\
\hline &
\end{tabular}


Table 3.1: Selected models for analysis of reproductive traits.

Table 3.2: Selected models for analysis of metabolic hormones and metabolites. 60

Table 3.3: Selected models for analysis of production traits

Table 3.4: Selected models for survival analysis of interval from calving to conception.....

Table 3.5: Crosstabulation of BCS at calving with diet

Table 3.6: Effect of milk progesterone profile on probability cows to be pregnant.

Table 3.7: Effect of BCS at calving on probability of cows to be pregnant and to express abnormal milk progesterone profiles.

Table 3.8: Effect of diet on probability of cows to be pregnant and to express abnormal milk progesterone profiles.

Table 3.9: Effect of diet and BCS at calving on days to first oestrus.

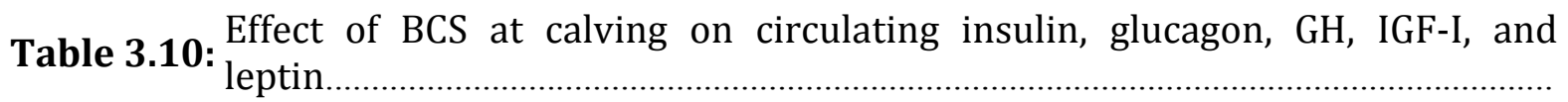

Table 3.11: Effect of diet on circulating insulin, glucagon, GH, IGF-I, and leptin

Table 3.12: Effect of BCS at calving on circulating glucose, urea, $\beta$-hydroxy butyrate, and non-esterified fatty acids

Table 3.13: Effect of diet on circulating glucose, urea, $\beta$-hydroxy butyrate, and nonesterified fatty acids

Table 3.14: Effect of BCS at calving on dry matter intake, live weight, milk yield, BCS, nadir BCS, nadir week, and postpartum body condition loss.

Table 3.15: Effect of diet on dry matter intake, live weight, milk yield, BCS, nadir BCS, nadir week, and postpartum body condition loss.

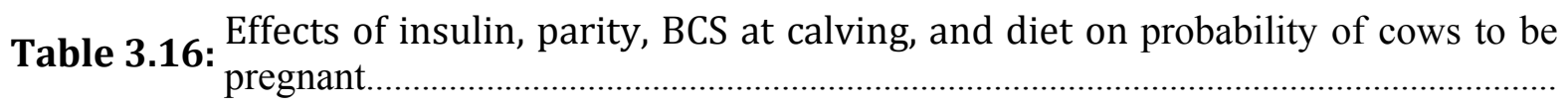

Table 3.17: Effects of insulin, IGF-I, glucose, parity, BCS at calving, and diet on milk progesterone profile...... 
Table 4.1: Selected models for analysis of the effect of lactational stage on circulating adiponectin, metabolites, metabolic hormones, and production traits.

Selected models for analysis of the effects of diet and BCS at calving on

Table 4.2: circulating adiponectin, metabolites, metabolic hormones, and production traits.

Table 4.3: Selected model for regression analysis of DMI

Table 4.4: Effect of lactational stage on circulating metabolic hormones.

Table 4.5: Effect of lactational stage on circulating metabolites

Table 4.6: Effect of lactational stage on productive traits

Table 4.7: Effect of diet and BCS at calving on circulating metabolic hormones.

Table 4.8: Effect of diet and BCS at calving on circulating metabolites.

Table 4.9: Effect of diet and BCS at calving on production traits

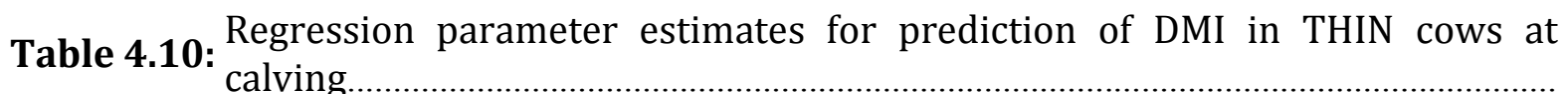

Table 4.11: Regression parameter estimates for prediction of DMI in FAT cows at calving.

Table 5.1: Selected models for analysis of the effect of circulating adiponectin levels on metabolites, metabolic hormones, and production traits.

Table 5.2: Selected models for analysis of reproductive traits.

Table 5.3: Selected models for analysis of milk progesterone profile and cystic body.

Table 5.4: Selected models for survival analysis of interval from calving to conception.

Table 5.5: Model fit criteria and acceptable fit interpretation...

Table 5.6: Effect of milk progesterone profile on probability for cows to be pregnant 
Table 5.7: Effect of circulating adiponectin levels on probability cows to be pregnant.

Table 5.8: Crosstabulation of circulating adiponectin levels with milk progesterone profile.

Table 5.9: Crosstabulation of circulating adiponectin levels with follicular cysts found at day 90 postpartum

Table 5.10: Effects of milk yield, DMI, GH, and parity on milk progesterone profile 165

Table 5.11: Effects of insulin, glucose, GH, NEFA, and parity on follicular cyst formation. 165

Table 5.12: Effects of insulin, milk yield, and IGF-I on days to conception 166

Table 5.13: Model fitted values for different insulin concentrations.

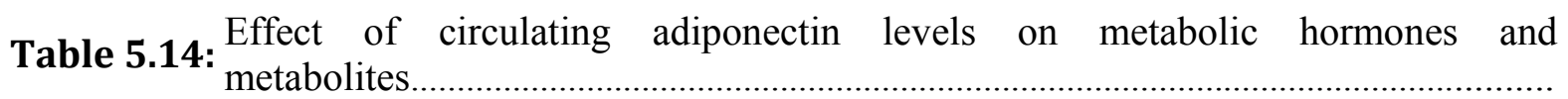

Table 5.15: Effect of circulating adiponectin levels on production traits

Table A.1: Formulation and composition of High Starch and High Fat diets.

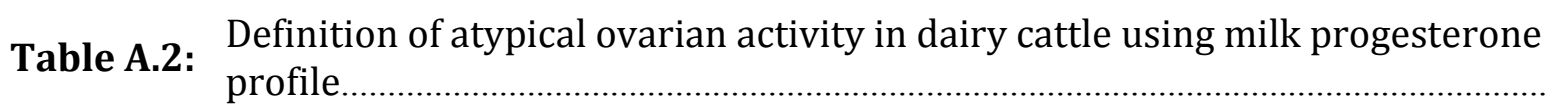

Table B.1: Model parameter estimates. 
Figure 2.1: BCS management in the modern dairy cow

Figure 2.2: Short term homeostatic regulation of circulating glucose by the coordinated action of pancreatic hormones

Figure 3.1: Calving-to-conception survival analysis curves for FAT and THIN cows at calving..

Figure 3.2: Calving-to-conception survival analysis curves for cows fed the high starch (HS) and the high fat (HF) diet.

Figure 3.3: Effect of circulating insulin on probability of cows to be pregnant.

Figure 3.4: Probability of cows to be pregnant adjusted for different condition status at calving (FAT versus THIN) and circulating insulin concentrations.

Figure 3.5: Probability of cows to be pregnant adjusted for different dietary treatments (HS versus HF) and circulating insulin concentrations.

Probability of cows to be pregnant adjusted for different condition status

Figure 3.6: at calving (FAT versus THIN), diets (HS versus HF), and circulating insulin concentrations.

Figure 3.7: Effect of circulating insulin on probability for cows to express abnormal milk progesterone profile.

Probability for cows to express abnormal milk progesterone profile

Figure 3.8: adjusted for different condition status at calving (FAT versus THIN) and circulating insulin concentrations

Probability for cows to express abnormal milk progesterone profile

Figure 3.9: adjusted for different dietary treatments (HS versus HF) and circulating insulin concentrations

Probability for cows to express abnormal milk progesterone profile

Figure 3.10: adjusted for different condition status at calving (FAT versus THIN), diets (HS versus $\mathrm{HF}$ ), and circulating insulin concentrations

Figure 4.1: Box -Whisker plot of circulating adiponectin values

Figure 4.2: Effect of lactational stage on circulating adiponectin. 
Figure 4.3: Relationships among DMI and circulating adiponectin, glucose, leptin, MEBAL, and milk yield in FAT and THIN cows at calving.

Figure 5.1: Scatter plot graphical representation of circulating adiponectin values by week of experiment.

Figure 5.2: Calving-to-conception survival analysis curves for cows with HIGH and 153

Figure 5.3: Effect of postpartum change in body condition score $(\triangle \mathrm{BCS})$ on probability of cows to be pregnant

Figure 5.4: Effect of circulating IGF-I on probability of cows to be pregnant.

Figure 5.5: Effect of days to first oestrus on probability of cows to express abnormal progesterone profiles

Figure 5.6: Effect of days to first oestrus on probability for cows to express cystic body.....

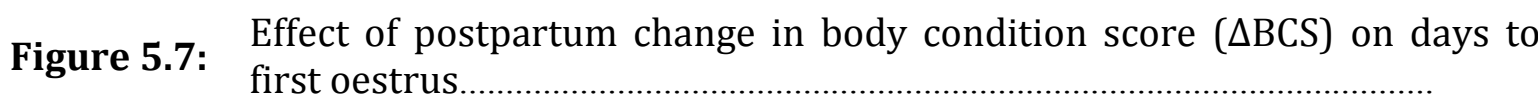

Figure 5.8: Effect of days to first oestrus on days to conception

Figure 5.9: Effect of circulating adiponectin on probability of cows to express abnormal progesterone profiles.

Figure 5.10: Effect of circulating leptin on days to first oestrus

Figure 5.11: Effect of milk yield on probability of cows to be pregnant.

Figure 5.12: The basic model: Glucose interrelationships with adiponectin, BCS, metabolites, and metabolic hormones.

Figure 5.13: Glucose interrelationships with adiponectin, BCS, metabolites, and metabolic hormones in cows with LOW previous milk production.

Figure 5.14: Glucose interrelationships with adiponectin, BCS, metabolites, and metabolic hormones in cows with HIGH previous milk production.

Figure 6.1: Overview of factors influenced reproductive indices and subsequent cow fertility in the present project... 
Figure A.1: $\quad$ Composite standard curve of plasma adiponectin assay.............................. $\quad 207$

Figure A.2: $\quad$ Standard curve of milk adiponectin assay....................................................... 209 


\section{Introduction and purpose of the study}

Milk yield is influenced by genetic, nutritional and environmental factors (Rook \& Thomas, 1983; Garnsworthy \& Webb, 1999). On a short term basis, the efficiency of nutrient use for milk production is primarily dependent on milk volume, but as milk yield increases a lower proportion of total feed intake is used for maintenance (Chilliard, 1992). Consequently, the modern high yielding cow loses weight and mobilizes adipose and muscle tissue depots to support milk production. Thus, high producing dairy cows experience a metabolic stress involving negative energy balance (NEB) in early lactation (Bauman \& Currie, 1980). The main characteristics of this condition are loss of body weight and mobilization of body fat stores because feed intake cannot support the energy required for milk yield and maintenance (Beam \& Butler, 1999; Butler, 2000; De Vries \& Veerkamp, 2000).

In general milk production and reproductive performance are highly negatively correlated in dairy cows (Macmillan et al., 1996; Royal et al., 2000; Stevenson, 2001; Lucy, 2002; Opsomer et al., 2006). In recent decades, genetic improvement in dairy cows has led to a dramatic increase in milk yield, which has been accompanied by an inherent decline in reproductive performance (Butler, 2000; Royal et al., 2000; Evans et al., 2006; Garnsworthy et al., 2008a). Probably, many other reasons (such as nutrition, management, and poor expression or detection of oestrus) are at least in the same degree accountable for the decreased reproduction performance in dairy cows (Garnsworthy \& Webb, 1999; Lucy, 2001; Dobson et al., 2007).

Nutrition interacts with reproductive performance and this interaction has prominent influences on livestock productivity and viability (Butler \& Smith, 1989; Garnsworthy \& Webb, 1999). This interaction is still being investigated, but it seems that the relationship between nutrition and reproduction is dynamic, complex, and not well understood (Gordon 1996; Boland \& Lonergan, 2003). The reproductive capacity of the postpartum cow is a critical element that determines overall biological and productive efficiency. Thus, failure 
of cows to resume cyclicity after calving is a critical point that influences the economical profitability of the cattle industry (Roberts et al., 1997; Stott et al., 1999; Evans et al., 2006).

Body condition score (BCS) is an indirect measure of nutritional status (Macmillan et al., 1996; Garnsworthy, 2006). It is well known that BCS of a cow, especially at certain points of its lactation cycle, directly influences fertility (Butler, 2003; Garnsworthy, 2006). This is true for a variety of important fertility indices, especially calving interval (Lucy, 2001; Oltenacu \& Algers, 2005; Dobson et al., 2007). BCS at calving is believed to influence reproductive performance due to its influence on tissue mobilization and on uterine involution. Moreover, BCS at calving is strongly related with condition loss and postpartum NEB, and influences circulating metabolic hormones and metabolites (Roche et al., 2009).

Adipose tissue releases a variety of adipokines, which are implicated in energy metabolism and reproduction. Leptin and adiponectin are two of the better-studied adipokines, and they are considered to have local and systemic effects (Antuna-Puente et al., 2008). Leptin is positively correlated with BCS, and NEB causes a notable reduction in plasma leptin concentrations (Chilliard et al. 2005). Moreover, leptin has been found to modulate nutrient transfer and partitioning by interaction with other hormones including insulin, glucagon, glucocorticoids, growth hormone $(\mathrm{GH})$, insulin-like growth factor I (IGF-I), cytokines and thyroid hormones (Hill, 2004; Garnsworthy et al., 2008a). Adiponectin expresses insulin-sensitizing actions and may be involved in insulin resistance and glucose homeostasis (Antuna-Puente et al., 2008). Fat mass may exert direct negative feedback on adiponectin secretion (Gordon et al., 2007) and a negative correlation between circulating adiponectin and fat mass has been reported in many animal and human models (Kadowaki \& Yamauchi, 2005). The link between leptin and reproduction is strong and well documented (Chilliard et al. 2005), whereas there is only limited evidence that adiponectin can directly regulate reproductive functions (Mitchell et al., 2005; Dupont et al., 2008). 
Insulin is a key hormone in endocrine control which facilitates movement of glucose across cell membranes, and thereby regulates the concentration of blood glucose (Guyton \& Hall, 2006). Glucose is the principal source of energy for life processes in the mammalian cell (Kaneko, 2008a). In postpartum cows, decreased insulin reduces glucose uptake by insulin-dependent organs such as adipose and muscle tissue, and increases glucose availability for the insulin-independent mammary gland (Bossaert et al., 2009). Decreased insulin (McGuire et al., 1995) and increased GH (Rhoads et al., 2004) concentrations also promote adipose tissue mobilization, resulting in elevated levels of circulating NEFA and BOHB, which favours milk yield. During early lactation, glucagon concentration increases relative to the dry period to stimulate lipolysis and gluconeogenesis, in order to provide the body with the required energy to support the high milk production (De Boer et al., 1985). Feeding dairy cows on diets to increase circulating insulin concentrations for the first 50 postpartum increased the proportion of cows ovulating during this period (Gong et al., 2002). Control of glucose homeostasis through dietary modulation of circulating insulin has been a key nutritional strategy to alleviate the postpartum dairy cow from the detrimental effects of NEB, and improve reproductive performance (Garnsworthy et al., 2009).

The poor fertility of high yielding dairy cows has been the main scientific focus for many research groups around the world. Because the genetic makeup of the modern high yielding cow is established and it is difficult to change in the short term, much attention has been paid to the interaction between nutrition, condition and adipose with reproductive performance. Work at the University of Nottingham, in collaboration with other researchers, addressed the problem of reduced fertility in dairy cows. The main output of this programme was the development of a nutritional strategy which possibly helps cows to improve fertility without depressing milk production (Gong et al., 2002; Adamiak et al., 2005, 2006; Fouladi-Nashta et al., 2005, 2007; Garnsworthy et al., 2008a, 2008b, 2008c, 2009). This $\mathrm{P} h \mathrm{D}$ project builds on that programme by utilising existing data and blood samples in further laboratory and data analyses. 
The overall hypothesis was that the reproductive performance of lactating dairy cows is affected by nutrition and body condition, and that the related hormonal and metabolic profile plays a role in cow fertility. Moreover, the present $\mathrm{P} h \mathrm{D}$ project was to study the effects of different nutritional and condition challenges on metabolic and hormonal profile, circulating adipokines, and reproductive performance in dairy cows. A special emphasis was directed to circulating adiponectin and its association with metabolic and hormonal status, and reproduction. Finally, the major scope of this project was to find evidence for the mechanism which possibly associates changes in body condition, diet, and physiological stage with changes in hormonal and metabolic profile. This mechanism is suggested to be implicated not only in nutrient partitioning but also in reproductive success or failure. 


\section{Review of literature}

\subsection{Postpartum nutritional strategies}

The interaction of nutrition with reproduction can be characterized as complicated, unstable, and variable (Boland et al., 2001). Such interaction involves both past and present nutritional status, but other factors, such as genetic makeup, animal condition, environmental influence, and physiological stage are determinant regulators of nutritional effects on reproductive performance (Short et al., 1990; Garnsworthy \& Webb, 1999; Lucy, 2001; Stevenson, 2001; Robinson et al., 2006). The detrimental impact of negative energy balance (NEB) on reproduction evidences how closely nutrition is related with reproduction (Nebel \& McGilliard, 1993; Butler \& Smith, 1997; Santos, 2007, 2009). Many nutritional approaches have been applied in periparturient cows in order to overcome the adverse consequences of NEB in reproduction, and to maximize cow profitability and welfare (Grummer, 1995; Friggens et al., 2004; Beever, 2006). Generally, such nutritional strategies are aimed at maintenance of body condition score (BCS) above the figure of 2.0 or better 2.5 throughout the year; BCS should not fall below 2.0 during the year, and BCS should be 2.5 to 3.0 at parturition (Garnsworthy, 2006). All approaches are based on the finding that fatter cows at calving eat less food and lose more body fat than thinner cows (Garnsworthy, 2006; Chagas et al., 2007). Given that a periparturient cow suffers from severe NEB due to decreased appetite and increased milk production (Grummer, 1995), most nutritional regimes aim at stimulating cow appetite or increasing the energy concentration of feeds, along with preserving high milk production. Consequently, these strategies often fail in terms of reproduction because they totally ignore the time taken for reproduction recovery after the calving (Beever, 2006).

More recently, researchers have attempted to find if high milk yield could be maintained along with high fertility rates in high yielding cows (Gong et al., 2002a, 2002b; FouladiNashta et al., 2005; Garnsworthy et al., 2008a, 2008b, 2008c, 2009). The most common strategy for reducing the degree of NEB in early lactation is to increase dietary energy 
concentration by increasing the starch or fat components of the ration at the expense of forage components (Garnsworthy et al., 2009). High fat diets with total fat concentration over $50 \mathrm{~g} / \mathrm{kg}$ of dry matter depressed plasma insulin concentration in cows at the onset of the breeding period (Garnsworthy et al., 2008b). Conversely, high starch diets induced high plasma insulin concentration and increased the proportion of cows ovulating within 50 days of calving, reduced the intervals from calving to first ovulation, and tended to reduce the intervals from calving to first service and to conception (Gong et al., 2002b). Moreover, Adamiak et al. $(2005,2006)$ and Fouladi-Nashta et al. (2005) demonstrated that diets designed to increase plasma insulin concentration had negative effects on blastocyst rate in heifers and in lactating cows respectively. In addition, Fouladi-Nashta et al. (2007) reported that diets with a high fat content had beneficial effects on blastocyst rate in lactating dairy cows, although they decreased circulating insulin. The main conclusion of all these studies is that dietary induced increases in insulin can be beneficial for resumption of oestrous cycles postpartum. However, high insulin concentration might have detrimental effects on oocyte developmental processes, basically at the stage of blastocyst. Garnsworthy et al. (2009) tested the hypothesis that pregnancy rate will be enhanced by feeding a diet that increased plasma insulin until cows resume ovarian cycles, and then switching to a diet that decreased plasma insulin during the mating period. This hypothesis was confirmed and it is a paradigm how nutritional strategies can impact on reproductive defects in high yielding cows without compromising animal welfare and productivity. 


\subsection{Body condition score and body condition score management in dairy cows}

Although live weight (LWT) change is a poor indicator of tissue mobilisation during early lactation, LWT is widely used as an index of tissue mobilisation. However, simultaneous increases in feed intake, gut contents, and water repletion of body tissues may disguise the real magnitude of tissue mobilisation measured by LWT (Sutter \& Beever, 2000; Garnsworthy, 2006; Bewley \& Schutz, 2008). Body condition score is a rapid, noninvasive, inexpensive, but subjective way of assessing body reserves of cow. It is easy applicable at the farm level and might give a more realistic view of the lipid and muscle reserves than LWT (Garnsworthy, 2006). Condition score assessment of cow is based on palpation and visual examination of the animal at certain anatomical and morphological body regions (i.e. assessing the level of fat cover over the transverse processes of the lumbar vertebrae and around the tailhead) (Roche et al., 2009). A variety of body condition scoring systems were introduced from 1970 to 1980 (Garnsworthy, 2006). All are based on the same basic principle of physical and visual examination of the animal, but different scales, anatomical regions, and emphasis weights on body reserves assessment are given. Garnsworthy (2006), Bewley \& Schutz (2008), and Roche et al. (2009) give a list of equations to convert BCS from other BCS systems to 1 to $5 \mathrm{UK} / \mathrm{US}$ system which is the most commonly used.

In recent decades, BCS has been proved a useful management tool for assessing nutritional status and energy balance (EB) during lactation in dairy cows. Estimates of BCS are significantly correlated with subcutaneous fat (Garnsworthy \& Topps, 1982; Domecq et al., 1995; Heuer et al., 1999; Garnsworthy, 2006). Ruegg \& Milton (1995) reported that excessive loss of BCS during early lactation was related to metabolic diseases and Gearhart et al. (1990) described BCS at calving as a risk factor for poor reproductive performance in dairy cows. However, the major drawback of BCS application and usefulness are; the scale of observations may be subjective; and BCS determined by different persons and in different studies are not directly comparable. Moreover, although BCS is positively correlated with LWT, in early lactation it is not unusual for cows to maintain or gain LWT whilst losing condition, due to the variability of gut contents 
(Garnsworthy, 1988; Bewley \& Schutz, 2008). It is known that subcutaneous fat correlates strongly with total body fat (Faulkner et al., 1990; Bewley \& Schutz, 2008). Thus, the disadvantages of BCS application can be overridden by using ultrasound technology to assess subcutaneous fat depth.

Absolute BCS at critical points of the lactation cycle (e.g. BCS at calving, nadir BCS, and BCS at drying-off) is as important as BCS change or loss, which is the difference between body condition scores assessed at two critical points (Bewley \& Schutz, 2008). It is well known that body condition loss and especially BCS at calving affect directly dry matter intake (DMI), NEB, fertility, milk yield and composition, and health of high yielding cows (Butler, 2003; Garnsworthy, 2006). Garnsworthy \& Topps (1982) studied the effect of BCS at calving on DMI and milk yield. Their study clearly indicated that fat cows at calving ate less and lost more condition than thin or moderate cows over the first 12 weeks of lactation. Many other studies (Broster \& Broster, 1998; Roche et al., 2009) have shown the negative correlation of BCS at calving and BCS loss with DMI in early lactation. It is now clear that over-conditioned cows at calving are prone to experience deeper and longer NEB and mobilize their body reserves due to reduced feed intake in early lactation. Consequently higher BCS at calving is not a recommended approach to alleviate cows from severe NEB postpartum because the unfavourable depression in DMI triggers longer and deeper NEB along with greater BCS loss (Garnsworthy, 2006).

Body condition score has been considered as an indirect measure of nutritional status (Short et al., 1990; Garnsworthy, 2006; Bewley \& Schutz, 2008). This implies that BCS is affected by diet composition and DMI (Short et al., 1990; Garnsworthy, 2006). According to Short et al. (1990) the effect of nutrition on reproduction depends on whether nutritional differences exist before or after calving. In addition, they stated that BCS at calving is more important than postpartum BCS loss, and consequently nutritional management of animals in the dry period is a crucial factor to ensure timely reproductive functionality after calving. This study also suggested that the relationship between postpartum anoestrus (PPA) and BCS at calving is nonlinear, and that BCS at calving influences postpartum DMI. The effect of different diet compositions on thin and fat cows was reviewed by 
Garnsworthy (2006). The main conclusion was that positive EB in early lactation is not unrealistic and it can be achieved under certain combination of BCS and dietary regimes (e.g. thin cows fed a high energy diet).

Milk production is the major objective of intensive dairy systems, but genetic improvement of cows for higher milk yield results in greater BCS loss in early lactation (Veerkamp et al., 2001, 2003; Dechow et al., 2002; Berry et al., 2003; Pryce \& Harris, 2006). Dechow et al. (2002) suggested that modern genetic selection should be directed to increase milk yield, but to minimize the BCS loss postpartum. Buckley et al. (2003) reported that for every $100 \mathrm{~kg}$ increase in genetic merit for milk yield, there is a loss of $0.02 \mathrm{BCS}$ units between calving and first service. Holstein-Friesian is the main breed used in dairy systems. Increasing the proportion of Holstein genes from 50 to $100 \%$ within this breed, results in a mean decrease of BCS by 1 unit (Veerkamp et al., 2001). Body condition score is related to milk composition. Pryce et al. (2001), Veerkamp et al. (2001) and Berry et al. (2003) proposed that there is a negative genetic correlation between BCS and fat and protein contents of milk. Roche et al. (2007a) found that butter fat was positively correlated with increasing calving and nadir BCS for a period from 60 to 270 days in milk, but milk protein was influenced positively by nadir BCS and negatively by BCS loss.

Poor or excessive BCS have been associated with health problems and decreased fertility in many studies. According to Mulligan et al. (2006a, 2006b), over-fat cows at calving had a greater probability of expressing fatty liver, ketosis, retained placenta, calving difficulties, and milk fever. Butler \& Smith (1989) and Villa-Godoy et al. (1990) demonstrated that when NEB coincides with excess BCS then the result is declined fertility. Over the last three decades numerous studies showed the negative impact of excess or inadequate BCS at calving, BCS loss, and nadir BCS on postpartum reproductive performance (Garnsworthy \& Topps, 1982; Heuer et al., 1999; Moreira et al., 2000; Yamada et al., 2003; Lopez-Gatius et al., 2003; Agenas et al., 2003; Roche et al., 2007b). The main conclusions from these studies were that reproductive indices determining reproductive efficiency of the herd, such as calving interval, days to first oestrus, days to first service, first service conception rate, and number of services, are related to BCS. 
Garnsworthy (2006) proposed that cows have a target BCS (about 2.5 units) that they try to attain in early lactation. Moreover, he stated that the biological drive for a cow to attain the target BCS appears to be as strong as the drive to attain a genetically-determined peak milk yield. Furthermore, the genetic correlation between BCS and milk yield is negative; the genetic correlations between health and fertility indices and milk yield are negative; and BCS loss is positively related to milk yield and negatively to health and fertility indices (Veerkamp et al., 2001; Berry et al., 2003; Pryce \& Harris, 2006). It is easily conceivable that there are marginal BCS targets to be achieved during the lactation cycle of the cow for satisfying health, milk yield, and reproductive efficiency.

According to Garnsworthy (2006) BCS management (Figure 2.1) must rely on the following basic principles:

1. Body condition score at calving should be allowed to vary no more than 0.5 BCS units above cow target BCS.

2. Cows of low genetic merit for milk yield (target BCS 2.5-3.0) should calve with BCS of 3.0 or less.

3. Cows of high genetic merit for milk yield (target BCS 2.0-2.25) should calve with BCS of 2.75 or less.

4. Body condition score should not fall below 2.0 during the whole lactation.

5. Body condition score at service should not fall below 2 to 2.5 .

According to Chagas et al. (2007) the perfect BCS fluctuation to minimize the effects of NEB on health and reproduction is BCS at calving 3.0-3.5 with a nadir BCS of 2.5-3.0. Mulligan et al. (2006b) suggested BCS targets of 2.75 at drying-off, 3.0 at calving, $\geq 2.75$ at service, and a nadir of 2.75. Coffey et al. (2004) suggested that if there is an ideal BCS or BCS loss, then they should be dependent on stage of lactation or/and the productive system under which cows are maintained. 


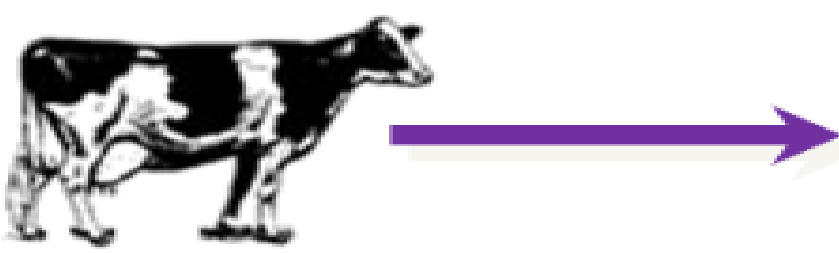

Genetically fatter dairy cow ancestor of $80 \mathrm{~s}$ with higher target BCS (3.0-3.5) in early lactation
Selection based on single milk yield trait and vast introduction of Holstein genes

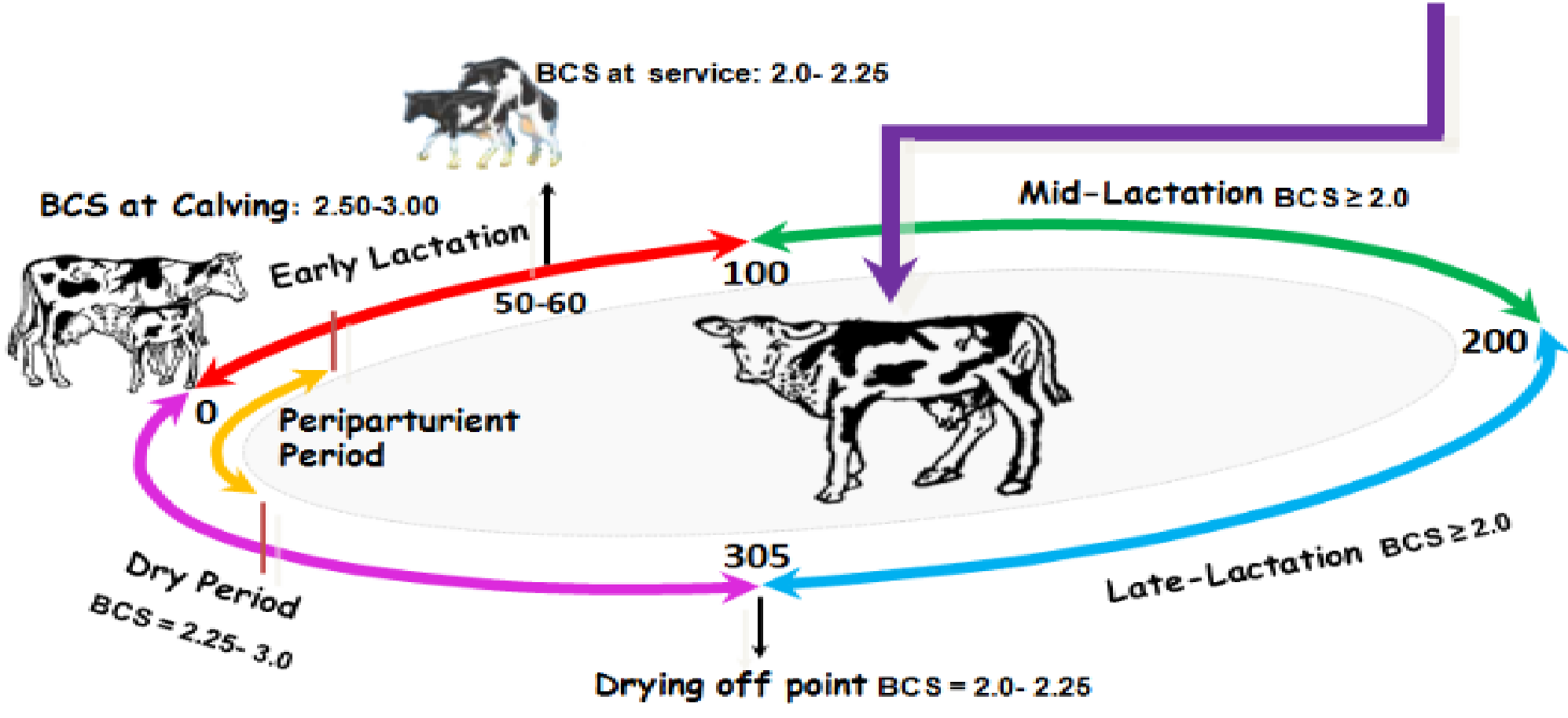

Figure 2.1: BCS management in the modern dairy cow (Based on the suggestions of Garnsworthy (2006), see text for details). 


\subsection{Glucose homeostasis and homeorhesis}

The energy contained in foods is made usable by the animal by intricate biochemical events that are generally defined as metabolism (Kaneko, 2008a). Traditionally, these biochemical events have been separated into the metabolism of three important ingredients of food; carbohydrates, proteins, and lipids (Guyton \& Hall, 2006). These also are the main constituents of animal body tissue and animal products (e.g. milk) (Bauman \& Currie 1980). The dominant circulating carbohydrate is glucose, which is the principal source of energy for life processes in the mammalian cell (Guyton \& Hall, 2006; Nafikov \& Beitz, 2007). Thus, the major function of glucose is to serve as an energy source for the animal cell, and its storage capacity (in the form of glycogen which is a large polymer of glucose) is nearly zero (lactating cows have the ability to store only $1 \%$ glucose in their livers in the form of glycogen) (Kaneko, 2008a). Cells require a constant supply of this essential nutrient, and only relatively small changes are tolerable without adverse effects on the health and productivity of the animal (Kaneko, 2008a; Nafikov \& Beitz, 2007). Fundamentally, glucose, protein and lipid metabolism are coupled to support the mammalian cell with a continuous constant flux of glucose which is tightly homeostatically and homeorhetically controlled (Bauman \& Currie, 1980). The liver plays a central role in buffering blood glucose concentration because it supplies and removes glucose from circulation. Hepatic glucose metabolism is mostly directed towards supplying rather than using glucose (Kaneko, 2008a; Ghanassia et al., 2007). Thus, the ruminant liver takes up only small amounts of glucose under normal physiological conditions and it is not an insulin dependent organ (Hayirli, 2006). On the other hand, nervous tissue, muscle tissue and the mammary gland during lactation are the primarily glucose-utilizing tissues of the animal (Brockman, 1978, 1979; Bauman \& Currie, 1980; Bauman, 2000; Reynolds, 2005). Muscle can deposit small quantities of glycogen, but is not able to liberate free glucose into the blood stream (without liver intervention) because of deficiency of the enzyme glucose-6-phosphatase (Kaneko, 2008a). Foetal tissue in pregnancy and the mammary gland during lactation are heavily dependent on maternal circulating glucose (Bauman \& Currie, 1980; Bell \& Bauman, 1997). The nutrient demands for pregnant animals at the end of pregnancy are $75 \%$ greater than those of non-pregnant animals of a 
similar LWT. The developing foetus, foetal membranes, gravid uterus, and mammary gland contribute to the increased glucose and nutrient demand (Bauman \& Currie, 1980). Homeothermy and antioxidant balance are also closely related to glucose homeostasis and homeorhesis (Ghanassia et al., 2007; Collier et al., 1982; Kadzere et al., 2002).

Centrally, there is a developed anatomical and functional network that monitors glucose availability that is involved in glucose homeostasis and food intake regulation (Bruning et al., 2000; Krasnow \& Steiner, 2006). Basic components of this centrally operating system are glucose-sensing neurons (Moran, 2010). These centrally placed glucose-sensing neurons are located at different spots of the brain (from the hindbrain to the hypothalamus) and together with peripheral glucose sensors, represent an integrated glucose monitoring system (Langhans \& Geary, 2010). Glucose phosphorylation by glucokinase is the ratelimiting step in ATP production and it is essential for the effects of glucose on membrane potential and ion channel function of glucose-sensing neurons (Moran, 2010). It is hypothesized that sensitive glucose-sensing neurons in the brain change their signalling in response to the presence (or the amount) of other metabolites and hormones such as insulin, leptin, non-esterified fatty acids (NEFA), and Amino Acids (AA). Glucose availability influences the expression and turnover of several catabolic and anabolic neuropeptides which presumably mediate the effects of glucose-sensing neurons on eating (Krasnow \& Steiner, 2006; Cone \& Elmquist, 2008; Langhans \& Geary, 2010).

Fatty acid oxidation (FAO) is closely related to glucose homeostasis and eating behaviour (Wade \& Schneider, 1992; Drackley, 1999; Ingvartsen \& Andersen, 2000; Allen \& Bradford, 2007). Inhibition of FAO is usually accompanied by a stimulation of eating in animals and man and FAO is tightly related to glucose availability by body tissues (Langhans \& Geary, 2010). Fatty acids and/or fatty acid metabolism can also be sensed centrally, in the mediobasal hypothalamus, and this also affects food consumption (Moran, 2010).

Cows are herbivorous and consume diets that are higher in structural carbohydrates and lower in fat (Lewis \& Hill, 1983; Allen et al., 2005). Nutrients absorbed by the cow differ 
markedly from those that are consumed because of the intervention of rumen microflora and the subsequent fermentation of organic matter (OM) of feed (Van Houtert et al., 1993). $\mathrm{OM}$ is partially fermented to volatile fatty acids (VFA: acetic, propionic, and butyric). Feed protein is partially degraded to AA and ammonia, which are incorporated into microbial protein of high biological value (Allen et al., 2005). Moreover, unsaturated fatty acids of feed are biohydrogenated and isomerized to varying degrees (Lewis \& Hill, 1983). The major fuels for cows are; VFA from ruminal and intestinal fermentation of OM; glucose from starch digestion in the small intestine (metabolized primarily to lactate by intestinal tissue); NEFA and AA absorbed from the gastrointestinal tract and mobilized from body reserves (Lewis \& Hill, 1983; Nafikov \& Beitz, 2007). Many ruminant tissues preferentially utilize acetate rather than glucose, but the mammary gland requires glucose, and large quantities are required for milk lactose synthesis (Reynolds, 2005). Insulin induced hypoglycaemia in lactating goats and cows resulted in a depression of milk yield (Smith, 1971).

The liver is the predominant site of glucose synthesis in the ruminant. Hexokinase activity is low in bovine liver and gluconeogenic capacity is high (Brockman, 1978). Consequently, for meeting the high demands of mammary epithelial cells for glucose precursors, net hepatic glucose production is over $4 \mathrm{~kg} / \mathrm{d}$ for cows producing about $60 \mathrm{~kg} / \mathrm{d}$ of milk (Reynolds, 2005; Nafikov \& Beitz, 2007). An interesting difference between ruminants and pigs is that when in positive $\mathrm{EB}$, gluconeogenesis and lipogenesis increase in ruminants but only lipogenesis increases in pigs (Nafikov \& Beitz, 2007). Ruminants normally absorb little glucose, and propionate is the primary gluconeogenic substrate. Almost all propionate is taken up by the liver and used for glucose synthesis (Allen et al., 2005). Propionate is responsible for as much as $80 \%$ of glucose produced in lactating cows. Underfeeding decreases utilization of propionate and other precursors derived from the diet, while the relative usage of lactate, glycerol and AA as gluconeogenic substrates increases. Most AAs are potential gluconeogenic substrates and they are considered to be the second most important glucose precursor (Reynolds, 2005; Nafikov \& Beitz, 2007). NEFA is the major source of energy in bovine liver, but the liver has limited capacity for fatty acid synthesis or triglyceride export (Allen et al., 2005; Nafikov \& Beitz, 2007). 
Milk yield exerts a pronounced drain on glucose and energy resources (Nafikov \& Beitz, 2007). In some studies, dairy cows selected for milk yield had lower circulating glucose (Harrison et al., 1990; Gutierrez et al., 1999), but this finding was not in line with other studies (Barnes et al., 1985; Westwood et al., 2000). Lukes et al. (1989) reported that glucose concentrations in high yielding cows are typically low at parturition. Moreover, the lower circulating glucose in higher genetic merit cows was observed later in lactation and lasted longer than in lower genetic merit cows (Westwood et al., 2000). In terms of circulating glucose, genetic merit for milk yield and underfeeding seem to interact (Veerkamp et al., 2003).

In dairy cows, two types of physiological regulation are instrumental to metabolic coordination during the transition period: homeostasis, which is the maintenance of physiological equilibrium, and homeorhesis, which is the orchestrated change in metabolism necessary to support a successful transition from one physiological state to another (Bauman \& Currie, 1980; Bauman, 2000). Given the species of the animal and the genetic make-up, environmental factors (such as management, nutrition, disease) can modify the interaction between homeostatic and homeorhetic circuits. In many cases of improper animal management, the homeostatic control for survival dominates the homeorhetic mechanism which is operated to support a physiological function (e.g. lactation or reproduction), resulting in a problematic or incomplete adaptation of the organism from one state to another (Bauman \& Currie, 1980; Friggens, 2003). Such maladaptations are common in transition cows when animals, in a short time, need to adapt to three completely different conditions (parturition, lactation, and reproductive recovery) (Grummer, 1993; Bell, 1995; Bell \& Bauman, 1997; Beever, 2006).

Regulation of glucose metabolism and nutrient partitioning involve interaction between homeorhetic and homeostatic circuits to ensure maintenance, production, and reproduction of the animal. The metabolic adaptations support the successful transition to lactation, leading to increased glucose synthesis by the liver and to decreased glucose oxidation by peripheral tissues at the beginning of lactation. However, sometimes the great demand of glucose is not achieved by the cow, resulting in reproductive failure, decreased 
productivity, and increased health problems (Bauman, 2000; Bauman et al., 2004). Nutrient partitioning and glucose economy are tightly endocrinologically regulated and this involves coordination of many organs, tissues, metabolites, and hormones (Bell \& Bauman, 1997; Leroy et al., 2008, 2010). Pancreatic hormones, adipokines, growth hormone $(\mathrm{GH})$, and their relationships appear to play a key role in regulating glucose homeostasis and homeorhesis in periparturient cows. These hormones will be discussed in the next section. 


\subsection{Pancreatic hormones and adipokines}

\subsubsection{Insulin}

Pancreatic hormones play a key role in endocrine regulation and facilitate the movement of glucose across cell membranes, thereby adjusting the concentration of blood glucose (Brockman 1978, 1979; Sasaki, 2002). When food is consumed by an animal, especially carbohydrates, insulin is secreted (Marieb \& Hoehn, 2007) and there is no circadian pattern of insulin secretion in ruminants (Trenkle, 1978). In general, circulating insulin is increased by feeding diets rich in starches but it is decreased by feeding diets rich in fat. However, the factors that influence insulin release are poorly documented in cows (Bossaert et al., 2008). Insulin plays an important role in storing excess energy and regulates glucose homeostasis along with its counterpart glucagon, (Figure 2.2) (Marieb \& Hoehn, 2007; Cryer, 2008, Guyton \& Hall, 2006). Nevertheless, the role of insulin is not constrained only to regulate glucose homeostasis and homeorhesis. Insulin interrelationships with $\mathrm{GH}$, insulin like growth factor- I (IGF-I), ovarian function and steroidogenesis are partially understood (Webb et al., 2004; Webb et al., 2007). Milk yield and milk composition are indirectly associated with the dynamics of nutrient availability in mammary gland (Veerkamp et al., 2003), whereas nutrient partitioning is mostly governed by insulin (Hayirli, 2006). Also, milk composition may or may not be controlled by insulin, and it is uncertain if selection for milk yield has any influence on insulin secretion, clearance and responsiveness in the modern dairy cow (Veerkamp et al., 2003).

\subsubsection{Insulin receptor and glucose transporters}

The insulin receptor is ubiquitous but liver, muscle tissue, and adipose tissue are the main areas exhibiting insulin bioactivity (Etherton, 1982). The insulin receptor is a transmembrane glycoprotein which belongs to the large class of tyrosine kinase receptors and consists of two $\alpha$ subunits and two $\beta$ subunits (Sasaki, 2002). Insulin receptors are embedded in cell plasma membranes of insulin dependent tissues, with the $\beta$ subunits 
passing through the cellular membrane, and $\alpha$ and $\beta$ subunits are linked by disulphide bonds (Kido et al., 2001; Sesti et al., 2001; Youngren, 2007). The binding of insulin to its receptor is the first step of a signalling pathway, triggering the consumption and the metabolism of glucose (Sasaki, 2002). Bound by insulin, the insulin receptor is phosphorylated from ATP to several proteins in the cytoplasm, including insulin receptor substrates (IRS-1 and IRS-2). This latter step activates phosphatidylinositol 3-kinase (PI-3K) and leads to an increase in glucose transporter 4 (GLUT4) molecules in the outer membrane of myocytes and adipocytes. By this mechanism, there is finally an increase in uptake of glucose from blood into muscle and adipose tissue (Kido et al., 2001; Sesti et al., 2001; Ben-Shlomo, 2002; Youngren, 2007). According to Sasaki (2002) possession of insulin receptor by tissue cells does not necessarily mean the tissue cells are responsive to insulin. For instance, bovine mammary epithelial cells have insulin receptors during lactation, but the tissue appears to be inert to insulin challenges (Laarveld et al., 1985). Furthermore, overexposure of adipose cells to high concentration of insulin, downregulates expression of insulin receptors, and leads to a loss of receptors from the membrane surfaces (Sasaki, 2002).

The uptake of glucose by tissue cells occurs by facilitating diffusion and it requires a carrier protein known as glucose transport (GLUT) (Klip et al., 1994; Thorens, 1996; Goodyear \& Kahn, 1998). GLUT in the plasma membrane exists in two conformational states. Extracellular glucose binds to the GLUT, which then alters its shape, discharging glucose in to the cell. Two types of GLUT are recognized (Thorens, 1996):

\section{(i) Insulin Independent Glucose Transporters}

GLUT1 is ubiquitously distributed in various tissues (erythrocyte, brain cells, mammary epithelial cells, adipose and muscle cells, and endothelial cells)

GLUT2 (liver, intestine, pancreatic $\beta$ cells and kidney)

GLUT3 (brain and/or foetal muscle cells)

GLUT5 (intestine)

(ii) Insulin dependent glucose transporters 
GLUT4 is primarily located in insulin-sensitive tissues, such as skeletal muscle, cardiac muscle, and adipose tissue.

Different tissues contain different types of GLUT (Herman \& Kahn, 2006). The tissue specific allocation of GLUT determines tissue responsiveness to insulin for uptake of glucose (Klip et al., 1994). Among these, only GLUT4 is found in insulin-sensitive tissues (muscle and adipose tissue) and requires insulin for uptake of glucose (Sasaki, 2002). In the bovine follicle and corpus luteum GLUT1 and GLUT3 act as the major transporters of glucose, although GLUT4 may play a supporting role (Nishimoto et al., 2006). GLUT1 is the major glucose transporter of lactating mammary epithelial cells (Bell \& Bauman, 1997). GLUT1 expression level was decreased from early to late lactation and become barely detectable in the non-lactating udder (Zhao \& Keating, 2007). According to Hayirli (2006) exogenous insulin may decrease availability of glucose for mammary tissue because insulin does not regulate uptake of glucose through GLUT1.

\subsubsection{Effects of Insulin on glucose metabolism and body reserves}

In general, insulin plays numerous roles in metabolism of carbohydrates, lipids, and proteins in adipose tissue, muscle, and liver in mammals (Aronoff et al., 2004). It is the main anabolic hormone in ruminants and acts to preserve nutrients in favour of the animal by directing feed nutrients to body stores rather than to milk production (Brockman, 1978, 1979; Brockman \& Laarveld, 1986). The hypoglycaemic effect of insulin action is antagonized by glucagon, adrenalin, glucocorticoids, and GH in cattle (Veerkamp et al., 2003). Plasma insulin concentration is negatively correlated with NEB in postpartum cows (Jorritsma et al., 2003) and insulin treatment increases feed intake, LWT, and body fat (Wade \& Schneider, 1992). The most important effect of insulin in mammals is its interplay with glycogen (Aronoff et al., 2004). This short term regulatory mechanism is critical for survival of the animal. After a meal, glucose is absorbed and insulin causes its storage in the liver in the form of glycogen. Between meals, when blood glucose concentration falls, insulin secretion decreases rapidly and liver glycogen splits back into 
glucose. Glucose is released back into the blood stream and prevents glucose concentration from falling too low (Cryer, 2008; Guyton \& Hall, 2006). This mechanism of controlling glucose homeostasis is stepwise and depends on the simultaneous activation and inactivation of several enzymatic circuits in the liver, including inactivation of liver phosphorilase, activation of glucokinase, and increased activity of the glycogen synthase (Allen \& Bradford, 2007; Vernon, 2005; Kokta et al., 2004; Guyton \& Hall, 2006). Another mechanism which is critical in glucose homeostasis is gluconeogenesis. Insulin is a potent inhibitor of gluconeogenesis by decreasing the quantities and activities of the liver enzymes which are involved in gluconeogenesis (Hayirli, 2006). Therefore, the main impact of insulin on glucose homeostasis is the control of two separate pathways, glyconeogenesis and gluconeogenesis (Aronoff et al., 2004; Nafikov \& Beitz, 2007).

Insulin was the first hormone discovered to have an impact on adipose tissue depots (Kokta et al., 2004; Cryer, 2008). Generally, insulin acts as fat sparer, and this effect is serious and just as important as the control of glucose homeostasis (Guyton \& Hall, 2006). In fact, glucose homeostasis and adipose tissue are linked under the spectrum of insulin activity (Ghanassia et al., 2007). That is because insulin increases uptake of glucose by most of the body tissues and automatically damps down lipid usage. In adipose tissue, insulin facilitates entry of glucose into adipose cells through GLUT4 (Hayirli, 2006). In turn, glucose is oxidized to form $\alpha$-glycerophosphate, which is used for esterification of free fatty acids during lipogenesis. Furthermore, insulin promotes fatty acid synthesis and storage in fat tissue depots, especially when dietary carbohydrate intake is high (Kokta et al., 2004; Guyton \& Hall, 2006). The mechanism by which insulin exerts its effect on adipose tissue is not clearly elucidated. However, this mechanism is believed to be based on the capacity of insulin to influence the activity of major enzymatic cascades such as hormone sensitive lipase (HSL) (insulin blocked), lipoprotein lipase (LPL) (insulin activated), and acetyl-CoA carboxylase (insulin activated) (Carmen \& Victor, 2006; Guyton \& Hall, 2006). Moreover, in adipose and muscle tissue, insulin stimulates triacylglyceride (TAG) synthesis by supplying fatty acids through stimulation of LPL (Ghanassia et al., 2007). Additionally, insulin suppresses lipolysis by lowering the level of cAMP and inhibits the activity of protein kinase $\mathrm{A}$ and hormone sensitive-lipase 
(Ghanassia et al., 2007; Brockman, 1978, 1979). Unlike non-ruminants, in which glucose is the main precursor for lipogenesis in adipose tissue, ruminants utilize acetate for the same purpose (Vernon \& Pond, 1997; Nafikov \& Beitz, 2007). Although precursors for lipogenesis are different, the stimulatory role of insulin on lipogenesis in adipose tissue of ruminants and non-ruminants is the same as in hepatic lipogenesis (Brockman \& Laarveld, 1986).

In the liver, insulin promotes lipogenesis and inhibits ketogenesis in both ruminants and non-ruminants (Brockman, 1978, 1979). Free fatty acids are taken up by the liver and are re-esterified with glycerophosphate, which is derived either from insulin-stimulated glycolysis or from glycerol formed by glycerophosphate kinase. Mitochondrial acetyl$\mathrm{CoA}$, which is generated by pyruvate dehydrogenase during glycolysis is transferred to the cytoplasm and converted to malonyl-CoA by acetyl-CoA carboxylase (Vernon \& Pond, 1997; Vernon, 2005). This is the rate limiting-step for hepatic lipogenesis and it is stimulated by insulin. Insulin exerts its antiketogenic effect on the liver by decreasing NEFA uptake (Grummer, 1993; Jorritsma et al., 2003). Insulin accomplishes this by altering lipogenesis to lipolysis ratio in adipose tissue in favour of lipogenesis, by increasing peripheral tissue ketone usage, by altering enzyme activities (carnitine palmitoyltransferase-I and acetyl-CoA carboxylase), and by changing the availability of substrates (malonyl-CoA) involved in ketogenesis in the liver (Brockman, 1979; Vernon \& Pond, 1997; Hayirli, 2006; Vazquez-Vela et al., 2008).

The ability of insulin to lower blood glucose concentrations is impaired during late pregnancy and early lactation in small ruminants (Prior \& Christenson, 1978; Debras et al., 1989). This phenomenon is defined as insulin resistance and it is commonly observed in cattle (Sano et al., 1991, 1993). Insulin resistance is the state in which physiological levels of insulin produce less potent biological responses (Pasquali, 2006; Zavalza-Gomez et al., 2008) due to decreased insulin responsiveness to glucose and/ or increased metabolic clearance (Sano et al., 1993). The molecular mechanism of insulin resistance may be placed at; (1) pre-receptor level, which includes decreased insulin production, increased insulin degradation, or both; (2) receptor level, which includes decreased number of 
receptors and decreased binding affinity; and (3) post-receptor level, which includes impaired intracellular signalling and translocation of GLUT (Hayirli, 2006). The current evidence suggests that the number and affinity of insulin receptors in adipose and muscle cells do not change during lactation and that the defect may be downstream of the insulin receptors (Ben-Shlomo, 2002; Barbour et al., 2007; Zavalza-Gomez et al., 2008). Glucose utilization by insulin dependent tissues is lower in pregnant animals than in non-pregnant and lactating animals (Nieuwenhuizen et al., 1998), and ketosis and ketoacidosis decrease tissue responsiveness to insulin in cows (Sakai et al., 1993; Steen et al., 1997). During late pregnancy and early lactation, the cow directs nutrients towards embryo and mammary tissue at a high-level metabolic priority (Bauman \& Currie, 1980; Vernon \& Pond, 1997). This priority coincides with lowered sensitivity of extra-hepatic tissues to insulin (Sano et al., 1991, 1993; Nieuwenhuizen et al., 1997, 1998), which presumably plays a key role in the development of hepatic lipidosis and ketosis (Steen et al., 1997). Gestational and lactational insulin resistance may be a homeorhetic adaptation that serves successful development of the foetus and survival of the neonate (Bauman \& Currie, 1980; Schoenberg \& Overton, 2010). Thus, the term insulin resistance may be misleading for characterizing this unique and physiological short-term condition in periparturient cows, because it may falsely refer to complications or diseases in other species that are largely irrelevant to the periparturient bovine model (Schoenberg \& Overton, 2010).

Circulating insulin and cerebrospinal levels of insulin are correlated with body adiposity and insulin receptors are present in the brain and hypothalamus of many species (Havrankova et al., 1979; Masters et al., 1987; Bruning et al., 2000), and cattle (Miller et al., 1994; Liu et al., 2009). Male and female mice with genetic deletions of central insulin receptors are obese (Moran, 2010), indicating that insulin receptor signalling in the brain is important for the control of LWT. Insulin is a potent regulator of feed intake and nutrient partitioning in ruminants (Ingvartsen \& Andersen, 2000). Moreover, insulin increases VFA absorption indirectly by stimulating growth of gut papillae. This latter effect may prevent accumulation of VFA, which stabilizes ruminal $\mathrm{pH}$ and allows greater feed intake (Hayirli et al., 2002). According to Hayirli (2006) insulin in ruminants interacts directly with other 
metabolites to regulate feed intake, but ruminants do not overcome hypoglycaemia simply by increasing feed intake.

\subsubsection{Effects of Insulin on milk yield and milk composition}

Insulin is postulated to play an indirect but very important role in milk yield volume and composition due to its regulatory control in nutrient partitioning (Herbein et al., 1985; Veerkamp et al., 2003). Low insulin decreases the uptake of glucose by insulin-sensitive tissues and enhances glucose availability for the mammary gland, which is insulininsensitive (Rose et al., 1997; Bossaert et al., 2008). Low circulating insulin is genotypically (Veerakamp et al., 2003; Gutierrez et al., 2006) and phenotypically associated (Veerakamp et al., 2003) with high milk yield, and insulin has been shown to be essential for ruminant mammary tissue development and cell maintenance in vitro (Collier et al., 1977; Mackle et al., 2002; Neville, 2006). Correlations between insulin levels and breeding values for milk production, butterfat and milk protein have been reported to be positive (Veerkamp et al., 2003). However, circulating insulin is unchanged between high yielding and low yielding cows (Lukes et al., 1989; Westwood et al., 2000) or even low in high genetic merit cows (Gutierrez et al., 1999; Gong et al., 2002b). Circulating insulin is lower in high yielding dairy cows than in beef cows, and this difference was probably due to decreased insulin secretion in the dairy cows (Veerkamp et al., 2003).

The glucogenic-insulin theory is based on the effects of insulin on nutrient partitioning. This theory explains, at least partially, milk fat depression and proposes regulatory influences of insulin on butterfat variation in milk (Griinari et al., 1996; Bauman \& Griinari, 2001). Insulin stimulates fat deposition and inhibits fat mobilization, but the ruminant mammary gland is insensitive to changes in circulating insulin. Milk fat depression occurs when cows consume diets high in digestible carbohydrates and low in fibre, which generate large quantities of propionate and glucose (Bauman \& Griinari, 2001; Rudolph et al., 2007). High circulating glucose induces release of insulin from the pancreas. Increased circulating insulin (two- to five-fold normal) inhibits fat mobilization 
in adipose stores (Vernon \& Pond, 1997; Bauman \& Griinari, 2001). Lipogenic precursors such as acetate, beta hydroxy butyrate, and diet-derived long chain fatty acids are preferably directed to adipose depots rather than to the mammary gland for butterfat synthesis. Finally, these changes result in a lack of lipogenic precursors for milk fat synthesis and depression of butterfat in milk (Bauman \& Griinari, 2001).

In general, insulin increases AA transport and protein synthesis (Veerkamp et al., 2003). Circulating AA has been shown to be the major precursors for milk protein synthesis (Petitclerc et al., 2000; Baldi et al., 2008). It has previously been demonstrated that lactating and non-lactating ruminants respond in the same way to exogenous insulin administration by decreasing circulating AA (Brockman, 1978; McGuire et al., 1995; Griinari et al., 1997; Mackle et al., 1999). Therefore, there is no evidence to suggest that insulin acts directly on the mammary gland to stimulate AA uptake. The decrease in circulating AA may be caused by the effect of insulin on other tissues, such as liver and muscle. In some studies using the hyper-insulinaemic euglycaemic clamp technique in lactating cows (McGuire et al., 1995, Griinari et al., 1997; Mackle et al., 2000), decreases in circulating AA was associated with increases in milk protein content. However, this needs to be further elucidated.

It has been shown that short term changes in circulating insulin have no direct effect on glucose uptake or milk synthesis by mammary epithelial cells in lactating ruminants (Laarveld et al., 1985; Nielsen et al., 2001). The mammary gland cannot synthesize lactose from any precursor except from glucose (Ohtani et al., 2011). Moreover, there was no effect of insulin administration on mammary gland glucose uptake or lactose synthesis and glucose turnover in the mammary gland (Brockman, 1979). According to Kronfeld (1963), variations in circulating insulin might occur without observable changes in mammary consumption of glucose, but low circulating glucose is a more direct cause of milk depression than insulin. 


\subsubsection{Insulin and the GH/IGF-I system}

Insulin may regulate the efficiency of GH signalling in liver and extrahepatic tissues of dairy cows (Rhoads et al., 2004). Secretion of GH is elevated in early lactation, and the correlation of insulin with GH is negative in dairy cows (Block et al., 2001; Veerkamp et al., 2003). Moreover, GH exerts two types of effects on adipose tissue and skeletal muscle; insulin-like and anti-insulin-like (Davidson, 1987). The insulin-like effects occur shortly after GH exposure and involve increased glucose usage and decreased lipolysis. When tissues undergo long-term exposure to $\mathrm{GH}$, cells responsive to insulin-like actions become unresponsive and this phenomenon is termed the refractory effect of GH (Mauras \& Haymond, 2005). The secretory pattern of GH throughout the day (biorhythm) is considered to cause a constant refractory state. The anti-insulin-like effects of GH are considered to better reflect the physiological action of the hormone. These effects include impaired glucose utilization, hyperglycaemia, stimulation of lipolysis, and induction and maintenance of the refractory state to insulin-like effects (Davidson, 1987; Mauras \& Haymond, 2005). Thus, insulin and GH have antagonistic roles in terms of glucose homeostasis, with minor insulin-like GH effects to serve possibly as parts of a homeorhetic and homeostatic mechanism (Bauman, 1999).

Growth hormone exerts its actions in almost every organ of the body, directly after binding to specific GH receptors (GHR) and/or indirectly after binding to GHR, stimulating the production of insulin-like growth factor-I (IGF-I) (Hull \& Harvey, 2000, 2001; Webb et al., 2004). The liver is the major source of circulating IGF-I (Rosen \& Pollak, 1999), but many tissues of the body, including ovary, uterus and mammary gland, can produce IGF-I (Hull \& Harvey, 2000, 2001, 2002; Webb et al., 2004). IGF-I serves as the main negative feedback signal on GH secretion under normal conditions in which GHR expression increases proportionally to circulating levels of GH (Hull \& Harvey, 2000, 2001, 2002). IGF-I controls cell growth, differentiation, and maintenance of differentiated cell function in many organs. IGF-I biological activity involves both insulin-like metabolic action and growth promoting action via endocrine, autocrine, and paracrine mechanisms (McGuire et al., 1992; Webb et al., 2004). IGF-I binds to specific cell surface receptors 
designated type I and type II IGF receptors with different affinity (Rajaram et al., 1997) and IGF-I can also interact with the insulin receptor (Rechler \& Nissley, 1985). Generally, pituitary derived $\mathrm{GH}$ or bovine somatotrophin (bST) administration stimulates the synthesis and secretion of hepatic IGF-I, but the combination of high GH and low IGF-I is common during the postpartum period and may be associated with NEB, underfeeding and low circulating insulin in dairy cows (Lucy, 2000; Veerkamp et al., 2003). Moreover, selection for milk production in dairy cows increases circulating GH whilst circulating IGF-I is lower for selected cows (Veerkamp et al., 2003).

In early lactating dairy cows, GH action to stimulate IGF-I synthesis is compromised, resulting in low circulating IGF-I (Vicini et al., 1991; Veerkamp et al., 2003; Rhoads et $a l ., 2004)$. Insulin may be involved in hepatic expression of GHR. It has been shown that chronically elevated circulating insulin increases plasma IGF-I in mid and late lactating dairy cows, and stimulates hepatic GHR gene expression (Mashek et al., 2001). Also, Rhoads et al. (2004) showed that there was a positive effect of insulin on GHR levels in liver and adipose tissue and that this positive effect was accompanied by an enhanced hepatic IGF-I synthesis in periparturient dairy cows. According to Veerkamp et al. (2003) the stimulatory effect of GH on IGF-I synthesis may be partly abolished during NEB when insulin levels are low. This might be because GH receptor numbers are decreased in the liver or because the function of GHR second messenger system has failed. According to Lucy (2008), as lactation progresses the somatotrophic axis recovers due to improved EB, increased circulating insulin, and enhanced expression of GHR in the liver.

\subsubsection{Insulin and reproduction}

Insulin also has the ability to relay metabolic information to the neuroendocrine pathways that influence reproduction and ovarian function (Krasnow \& Steiner, 2006; Garnsworthy et al., 2008a). Many observations from humans and animals with insulin-dependent diabetes mellitus or similar defects suggested that insulin plays a key role in reproduction (Veerkamp et al., 2003; Kalro, 2003; Krasnow \& Steiner, 2006). Rats bearing mutations to 
central receptors of insulin experienced reduced reproductive performance due to reduction in gonadotrophin secretion and impaired folliculogenesis (Bruning et al., 2000). Before the advent of insulin in 1921, pregnancies in women who had diabetes mellitus were rare (Kalro, 2003). Low circulating insulin, which may occur in diabetic patients, may also characterize high genetic merit dairy cows (Veerkamp et al., 2003). Low conception rate and low follicular phase progesterone levels have been associated with low circulating glucose and insulin in high genetic merit cows (Pryce et al., 1999; Snijders et al., 2001; Veerkamp et al., 2003; Webb et al., 2004).

Insulin plays roles in reproduction much more complicated than its glucose regulatory action, and it is implicated in follicular development and functionality (Veerkamp et al., 2003; Garnsworthy et al., 2008a). The ovary has been demonstrated to be the site of action for insulin and IGF-I in several species, including dairy cows (Spicer et al., 1990; Spicer \& Echternkamp, 1995; Webb et al., 2004; Webb et al., 2007; Velazquez et al., 2008). Growth factors such as insulin like growth factors (IGF), transforming growth factors (TGF), and epidermal growth factors (EGF) directly influence follicular growth by enhancing granulosa cell proliferation (Webb et al., 2004). The IGF family is likely to be important in the process of selection of dominant follicles by potentiating the action of folliclestimulating hormone (FSH) on granulosa cell differentiation, and by increasing the responsiveness of granulosa cells to FSH during final follicular growth (Garnsworthy \& Webb, 1999; Webb et al., 2004; Velazquez et al., 2008; Garnsworthy et al., 2008a). In the ovary, insulin is present in follicular fluid, insulin receptors are expressed by granulosa cells, and insulin stimulates ovarian steroidogenesis (Webb et al., 2004; Krasnow \& Steiner, 2006). In vitro insulin and/or IGF-I increase progesterone secretion in human and bovine granulosa cells (Spicer et al., 1993; Taketani et al., 2008), and IGF-I, along with gonadotrophins, stimulates ovarian steroidogenesis (Webb et al., 2004). This implies that the stimulatory effects of insulin on steroidogenesis, at least in part, may be exerted by the IGF-I receptor since IGF-I acts together with gonadotrophins to stimulate ovarian steroidogenesis (Webb et al., 2004). 
Several studies related changes in circulating insulin to alterations in pulsatile luteinizing hormone (LH) secretion (Downing \& Scarramuzi, 1997, Downing et al., 1999; Tanaka et al., 2000; Bucholtz et al., 2000). In vitro and in vivo, insulin stimulates GnRH release but this occurs only when glucose is available (Arias et al., 1992; Tanaka et al., 2000). On the one hand, short term food deprivation is associated with decreased circulating insulin and LH pulsatility. On the other hand, refeeding after food deprivation is accompanied by increases in both insulin and LH secretion (Krasnow \& Steiner, 2006). Thus, it appears that reduced insulin concentrations may signal the hypothalamus and pituitary inhibiting LH secretion during periods of limited feed intake (Wade \& Schneider, 1992). Furthermore, dietary induced increases in circulating insulin in early lactation cows may prevent the short luteal phase which is characteristic of the first postpartum oestrous cycle (Miyoshi et al., 2001). Moreover, insulin seems to influence embryo survival and ovulation in dairy cows. Adamiak et al. $(2005,2006)$ and Fouladi-Nashta et al. (2007) reported that diets designed to increase plasma insulin concentration had negative effects on blastocyst rate in heifers and in lactating cows, whereas Gong et al. (2002b) observed that the percentage of cows ovulating within 50 days postpartum increased from 55 to $90 \%$ in cows fed a high starch diet that induced higher insulin release in response to feeding. Garnsworthy et al. (2009), by adopting a feeding system that combined a glucogenic diet for the first 50 days postpartum and a lipogenic diet thereafter, demonstrated that insulin stimulus may play different roles in early lactation and in the breeding period in dairy cows.

Experiments in which animals were treated with insulin gave varying results with respect to reproduction (Krasnow \& Steiner, 2006). The interpretation of experiments in which insulin is injected peripherally is complicated due to the hypoglycaemia induced by administration of large doses of insulin (Hayirli, 2006). In many instances, adverse effects of systemic insulin treatment on reproductive function did not occur if normal circulating glucose was maintained (Downing et al., 1999). There are clear indications that postpartum glucose concentrations are lower in high genetic merit cows than in low genetic merit cows (Snijders et al., 2001; Veerkamp et al., 2003). McClure et al. (1967), Downie \& Gelman (1976) and Easdon et al. (1985) stated that low circulating glucose may be responsible for infertility in lactating cows and mice. In addition, pregnancy rate was higher in cows with 
high circulating glucose than in cows with low circulating glucose, and there was a trend for pregnancy rate to decline in cows with very high circulating glucose (Pehrson et al., 1992). Krasnow \& Steiner (2006) reviewed many experiments conducted with a variety of animal models in order to assess the implications of hypoglycaemia for reproduction. They reported that hypoglycaemia may reduce GnRH pulse generator activity, suppress pulsatile LH secretion, disrupt oestrous cyclicity and ovulation, decrease the magnitude of the steroid-induced LH surge, and impair mating behaviour. Selvaraju et al. (2002) reported that insulin and glucose concentrations were higher in cows which subsequently became pregnant than in non-pregnant animals. Downing et al. (1999) stated that there is a synergism between insulin and glucose at the ovarian level and it is likely the effects of short-term nutrition on ovulation rate may be mediated by direct ovarian actions of insulin and glucose. Mammals primarily rely on intracellular oxidation of glucose and fatty acids to get the energy necessary to support most physiological processes (Wade \& Schneider, 1992). Although peripheral tissues may utilize both glucose and fatty acids, the ovary uses glucose as its principal source of energy (Rabiee et al., 1999; Crooker et al., 2007; Scaramuzzi et al., 2010). Glucose is available for cellular oxidation and energy production not only when its quantity is sufficient in the body, but also when it is capable of entering cells, and that latter implies a role for insulin (Wade \& Schneider, 1992). It seems that the effect of glucose on fertility is primarily related to its properties as a metabolic fuel (Veerkamp et al., 2003). Insulin and functional insulin signalling are necessary for full reproductive competence. However, insulin by itself is usually insufficient to restore reproductive function in the face of extreme metabolic challenges, especially hypoglycaemia (Krasnow \& Steiner, 2006). This suggests that insulin might influence fertility mostly through metabolic fuel partitioning, mainly of glucose, rather than through direct effects (Veerkamp et al., 2003). Nowadays, it is believed that the follicle has a well developed system to sense glucose and nutritional status (Webb et al., 2004; Gransworthy et al., 2008a; Scaramuzzi et al., 2010). This system allows follicle to determine its growth and development, mainly by altering FSH induced effects on local oestradiol synthesis, in accordance with glucose availability (Webb et al., 2004; Scaramuzzi et al., 2010). 


\subsubsection{Glucagon}

Glucagon is released into the circulation when glucose is low (Brockman, 1978; Aronoff et al., 2004). The main physiological role of glucagon is to stimulate hepatic glucose output (glycogenolysis) leading to increased circulating glucose. In this way, Glucagon provides the major countregulatory mechanism for glucose homeostasis (Figure 2.2) (Aronoff et al., 2004). Another main effect of glucagon on glucose metabolism is stimulation of gluconeogenesis in the liver (Brockman, 1978; Jiang \& Zhang, 2003; Guyton \& Hall, 2006). Enhanced ability of the liver for gluconeogenesis in the presence of glucagon is related to increased activity of key gluconeogenic enzymes such as hepatic pyruvate carboxylase, and to high exportation rates of gluconeogenic substrates such as alanine, pyruvate, lactate, and glutamine (Roden \& Bernroider, 2003; Jiang \& Zhang, 2003; Aronoff et al., 2004). In adipose tissue there are two major effects of glucagon: (1) activation of adipose cell lipase that makes increased quantities of fatty acids available to other organs of the body; (2) inhibition of triglyceride storage in the liver that prevents the liver from removing fats from the blood stream. The latter makes additional amounts of fatty acids available for other energetic needs, for example milk production (Roden \& Bernroider, 2003; Jiang \& Zhang, 2003). In ruminants, the effect of glucagon on adipose tissue metabolism seems to be the same as with non-ruminants. Glucagon infusions resulted in increased mobilization of fatty acids and glycerol provided that insulin secretion was abolished (Brockman, 1978). However, a lipolytic role for glucagon in ruminants has not been confirmed in vitro. This suggests that if glucagon has an effect on adipose tissue, this is not as potent as the effect of insulin (Brockman, 1978, 1979). Glucagon has no direct effect on peripheral AA metabolism, but it promotes uptake of glucogenic aminoacids by the liver of sheep in vivo and enhances the conversion of alanine to glucose (Brockman, 1978). In vivo studies in sheep suggest that high concentrations of glucagon may be ketogenic. Glucagon might exert this action in the liver by activating carnitine acyltransferase reaction which is the first step of oxidation of fatty acids (Brockman, 1979). 


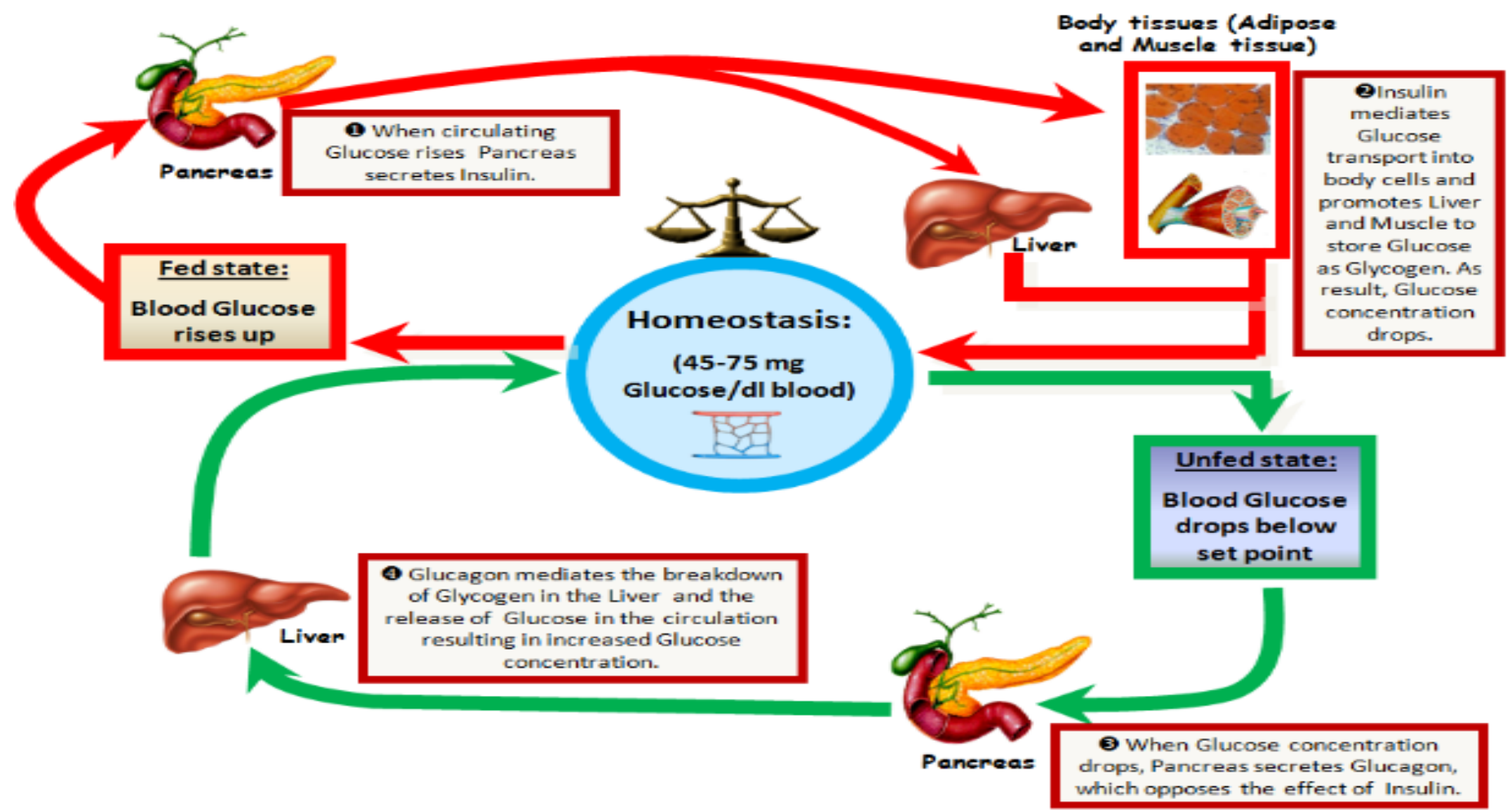

Figure 2.2: Short term homeostatic regulation of circulating glucose by the coordinated action of pancreatic hormones. Increased circulating glucose in the fed state stimulates secretion of the pancreatic hormone insulin by $\beta$ cells and subsequent release of insulin into the blood stream. Postprandially, a high concentration of insulin stimulates liver cells to increase glucose uptake and storage in the form of glycogen. Moreover, cells from insulin dependent tissues, mainly adipose tissue and muscle, increase glucose utilization and as a result circulating glucose declines to the set point and the insulin effect is diminished. Preprandially, when circulating glucose is low, $\alpha$ cells in the pancreas are stimulated to secrete the pancreatic hormone glucagon and gradually its circulating levels are increased. Glucagon exerts its effects mainly on the liver, where it increases glycogen breakdown resulting in increased circulating glucose. Shortly, after glucagon action circulating glucose increases to the set point and the glucagon effect is diminished (Adapted from Marieb \& Hoehn, 2007). 
Generally, insulin is the primary hormonal signal that controls lipid and ketone body metabolism in ruminants while glucagon has only secondary effects when circulating insulin is low (Brockman, 1979). Glucose availability has been implicated as a limiting factor for milk production and the ratio of insulin to glucagon during the lactation is believed to be strongly related to the metabolic processes facilitating milk yield (Herbein et al., 1985). According to De Boer et al. $(1985,1986)$, circulating glucagon increases from the dry period to early lactation in order to stimulate lipolysis and gluconeogenesis providing the body with the required energy to support high milk production. However, other studies (Herbein et al., 1985; Kokta et al., 2004) report that circulating glucagon does not change during lactation in dairy cows, suggesting almost minimal dependence of cows on gluconeogenesis. The role of glucagon stimulus has recently been revaluated in periparturient cows. Many studies were conducted to examine any positive effect of exogenous glucagon administration on postpartum NEB, glucose homeostasis, milk yield, and fatty liver syndrome (Steen et al., 1997; Bobe et al., 2003a, 2003b, 2004, 2007; Nafikov et al., 2006; Osman et al., 2008, 2010). The results of these studies indicated a positive influence of glucagon treatment on prevention of fatty liver without any effect on milk yield. 


\subsubsection{Leptin}

Adipose tissue is no longer considered to be just a store of dormant TAGs which are mobilized during NEB (Fruhbeck et al., 2001; Vernon, 2005; Ghanassia et al., 2007). TAGs are partly interchangeable with glucose, and in this way fat and glucose metabolism are coupled (Vernon, 2005; Marieb \& Hoehn, 2007). After the discovery of leptin, the scientific views about adipose tissue have changed considerably. Adipose tissue is now considered to be not only an active regulator of body weight but also an endocrine organ (Houseknecht et al., 1998; Williams et al., 2002; Macajova et al., 2004). It has recently become clear that lipid stores play a critical role in regulation of energy homeostasis, insulin action, and glucose metabolism (Yildiz \& Haznedaroglu, 2006; Kokta et al., 2004; Block et al., 2003). Moreover, the amount of body fat is the principal determinant of circulating levels of leptin. This means that there is a strong positive correlation between body fat and plasma leptin (Schneider, 2004; Zieba et al., 2005). Leptin is positively correlated to LWT and adiposity in ruminants. Also, leptin in growing ruminants is positively correlated with BCS when other confounding factors are absent. However, the correlation between leptin and BCS appears to weaken postpartum when many other physiological influences are in action (Hill, 2004).

Leptin effects can be divided to two main categories: (1) central effects in the brain, affecting the amount of food consumption, energy homeostasis, and reproduction; (2) peripheral effects in the majority of body tissues, affecting nutrient partitioning (Schneider, 2004; Macajova et al., 2004). Centrally, leptin exerts its function after binding to its long form receptor $\mathrm{Ob}-\mathrm{Rb}$. In the periphery, leptin action is inextricably associated with its binding to short form receptors (Ob-R) and there are at least six isoforms of $\mathrm{Ob}-\mathrm{R}$ (Houseknecht et al., 1998; Fruhbeck et al., 2001; Macajova et al., 2004; Liefers et al., 2005). Ob- $\mathrm{Rb}$ is the primary mediator of leptin actions because it is the only iso-form that is capable of relaying full downstream signalling pathways after binding of leptin. Ob-R mRNA is expressed in several organs (such as brain, liver, BAT and WAT, skeletal muscle, pituitary, ovary, testis, and uterus) where leptin may directly exert regulatory actions (Krasnow \& Steiner, 2006). Apart from this, short forms of leptin receptors 
circulate in the blood stream and bind leptin. This mechanism is believed to play a role in activation and inactivation of leptin molecule (Kokta et al., 2004; Houseknecht et al., 1998; Macajova et al., 2004).

Generally, the intricate influences of leptin on glucose homeostasis are exerted by the regulatory actions of leptin on feed intake, thermoregulation, lipid metabolism, and physical activity (Williams et al., 2002; Zieba et al., 2005; Liefers et al., 2005). Increases in adipose depots reflect elevated circulating leptin and enhanced transduction of leptin signalling in the brain. This results in decreased food intake and increased energy expenditure (Krasnow \& Steiner, 2006). Thus, the main central effect of leptin when it binds to $\mathrm{Ob}-\mathrm{R}$ in the brain is suppression of food intake and potential body weight loss due to increased metabolic rate (Hill, 2004; Krasnow \& Steiner, 2006). In cattle, expression of leptin mRNA and/or circulating leptin are rapidly decreased by restriction of energy intake, but are increased by refeeding (Chilliard et al., 2005; Krasnow \& Steiner, 2006). In the periphery, leptin: (1) stimulates free fatty acid (FFA) oxidation and increases glucose uptake and metabolism in skeletal muscles; (2) increases lipolysis in adipose tissue; (3) decreases glucose output and increases FFA oxidation in the liver (Yildiz \& Haznedaroglu, 2006; Kokta et al., 2004; Macajova et al., 2004). Interestingly, leptin has been found in milk (Weyermann et al., 2006; Martin et al., 2006). Although some studies have demonstrated leptin transfer from blood to milk by involving leptin receptors expressed by mammary epithelial cells, other studies have shown mammary synthesis of leptin (Bonnet et al., 2002). Whatever the mechanism, it is not known why leptin is secreted in milk or why its receptors exist in the mammary gland.Female $o b / o b$ mice usually fail to lactate when leptin treatment is ceased before parturition. Additionally, female $o b / o b$ mice treated with leptin for 14.5 days after successful mating were unable to lactate, even if leptin treatment was resumed after parturition. This implies a role of leptin in mammary gland development and lactation (Krasnow \& Steiner, 2006) but it needs to be further investigated.

Leptin may be modulated by insulin and GH, and conversely (Casanueva \& Dieguez, 1998; Williams et al., 2002; Zieba et al., 2005; Liefers et al., 2005). Generally, insulin 
stimulates circulating leptin in rodents and humans, but insulin effects on leptin in ruminants are unclear (Houseknecht et al., 2000; Block et al., 2001; Leury et al., 2003; Thorn et al., 2008). Insulin is a positive regulator of circulating leptin in lactating dairy cows in positive energy balance (Block et al., 2003) and this may imply that cellular energy availability is the primary factor regulating leptin synthesis (Block et al., 2001). However, the interaction between leptin and insulin could be characterized as quite complex and bidirectional (Yildiz \& Haznedaroglu, 2006). On the one hand, increased plasma leptin promotes peripheral insulin sensitivity. On the other hand, the same levels of circulating leptin reduce insulin secretion from pancreatic $\beta$ cells. This may suggest that there is a feedback loop between adipose tissue and pancreatic islets; the so-called "Adipoinsular axis". Any perturbation or deregulation of this axis is likely to contribute to development of certain pathologies such as insulin resistance, diabetes, and glucose intolerance (Yildiz \& Haznedaroglu, 2006; Block et al., 2003; Zieba et al., 2005). A recent study suggests that hypoglycaemia (induced by hyperinsulinaemia) inhibits rises in leptin in rats. This finding may support the hypothesis that falling glucose levels during a prolonged fast, directly or indirectly, signal the adipocyte to reduce leptin secretion (Yildiz \& Haznedaroglu, 2006).

Human obese patients showed decreased circulating GH and increased circulating leptin (Casanueva \& Dieguez, 1998). In contrast, acromegalic and anorexia nervosa patients expressed decreased circulating leptin and increased circulating GH (Popovic et al., 2001; Scacchi et al., 1999). Leifers et al. (2005) suggested that leptin expression in adipose tissue is possibly regulated in early lactating cows by the negative impact of GH. Moreover, restriction of energy intake stimulates GH secretion but suppresses leptin expression in human and ruminant adipose tissue (Block et al., 2001; Nagatani et al., 2000). According to Scacchi et al. (1999) and Dieguez et al. (2000) increased circulating free fatty acids and adiposity are mainly responsible for the inhibition of GH secretion. However, it is possible that leptin is another hormonal signal controlling GH secretion by acting on somatostatin and growth hormone releasing hormone (GHRH) producing neurons (Scacchi et al., 1999; Dieguez et al., 2000). Furthermore, GH prevents insulin-induced production of leptin by bovine white adipose tissue (WAT) explants (Houseknecht et al. 2000). In contrast, GH 
administration had no effect on circulating leptin during late pregnancy or early lactation in dairy cows (Leury et al., 2003; Block et al., 2003) and GH was potently stimulated by exogenous leptin administration in fasting sheep (Nagatani et al., 2000). Although leptin can act directly at the bovine anterior pituitary to modulate GH release, this effect is greatly dependent on nutritional status of the cow (Zieba et al., 2003). Moreover, GH treatment decreased circulating leptin only in pregnant dairy cows (Sauerwein et al., 2004). Therefore, the regulatory roles of GH on leptin and vice versa are debatable and largely dependent on the endocrine and the metabolic status of animal.

Much attention has been given to whether reproductive function and nutritional status are bridged by leptin (Krasnow \& Steiner, 2006). Leptin is a potent regulator of the hypothalamic pituitary ovary (HPO) axis and reproduction (Hill, 2004; Chilliard et al., 2005). In the leptin knockout mouse model, males and females are sterile, and treatment with leptin supplementation restores fertility (Hill, 2004). In vivo, leptin stimulates secretion of GnRH and gonadotrophins (Williams et al., 2002; Hill, 2004; Liefers et al., 2005; Krasnow \& Steiner, 2006), but in vitro inhibits steroidogenesis in the ovary and testis (Krasnow \& Steiner, 2006; Williams et al., 2002). In vivo, leptin is a potent stimulator of LH (Williams et al., 2002; Krasnow \& Steiner, 2006). In ruminants, central or peripheral supplementation of leptin to feed-deprived animals completely prevents the decreases in LH (Hill, 2004; Liefers et al., 2005; Chilliard et al., 2005). Leptin may serve as a trigger for sexual maturation or play a permissive role in initiation of the onset of puberty (Williams et al., 2002; Chilliard et al., 2005; Krasnow \& Steiner, 2006). Age at onset of puberty is affected by mostly feed intake, growth rate and adiposity (Moran et al. 1989). Elevated circulating leptin precedes sexual maturation in many animals, and it has been demonstrated that leptin plays at least a permissive role in onset of puberty (Ahima et al., 1997; Garcia et al., 2002). Leptin enhances bovine oocyte maturation (Paula-Lopes et $a l ., 2007)$ and improves the ability of the bovine oocyte to sustain embryonic development (Boelhauve et al., 2005).

Leptin roles are not restricted to regulation of reproductive function, but are extended to pregnancy and lactation (Hill, 2004; Chilliard et al., 2005). In many animals, including 
dairy cows, circulating leptin increases during pregnancy and reaches a peak just before parturition, at which point it starts to decrease rapidly (Chilliard et al., 2005; Krasnow \& Steiner, 2006). Normally, increased circulating leptin is accompanied by decreased food consumption, but because a sort of central leptin resistance is established, females of many species express hyperphagia (Hill, 2004; Krasnow \& Steiner, 2006). In the bovine, leptin resistance may be considered a successful homeorhetic adaptation because it allows pregnant females to consume greater quantities of feed than normal in order to support the great energetic demands of the ongoing foetal growth and the imminent lactation (Krasnow $\&$ Steiner, 2006). Feed intake is already low 2 weeks prepartum and further decreases postpartum in dairy cows (Allen \& Bradford, 2007). Therefore, modern periparturient dairy cows may be an exception displaying earlier hypophagia, although central leptin resistance has never been documented. 


\subsubsection{Adiponectin}

Adiponectin, also known as Acrp30, apM1, AdipoQ, and GBP28, is an adipokine secreted by adipose tissue (Fruhbeck et al., 2001; Kadowaki \& Yamauchi, 2005; Berg et al., 2002). Adiponectin protein form structurally belongs to the complement $1 q$ family, it is multimeric, and it is constituted by four main domains. Serum human or mouse adiponectin forms a variety of multimers from trimers to high molecular weight (HMW) multimers. Oligomerization, proteolytic cleavage, hydroxylation, and glycosylation of multimers are shown to play important roles in adiponectin activation and deactivation (Kadowaki \& Yamauchi, 2005). Adiponectin can exist as full-length or a smaller globular fragment, however, almost all circulating adiponectin appears to be as full-length adiponectin in plasma (Kadowaki \& Yamauchi, 2005; Garaulet et al., 2007; Giannessi et $a l$., 2007). It has been postulated that the different adiponectin multimers exert diverse effects in various tissues. Thus, adiponectin oligomerization is of high importance and it must be considered when adiponectin function is studied in a specific tissue (Garaulet et al., 2007; Shetty et al., 2009).

Binding of adiponectin to its receptors is the first step of its biological action. Two kinds of distinct but structurally related adiponectin receptors have been identified (AdipoR1 and AdipoR2) and they both bind globular and full-length adiponectin with different affinities (Ahima, 2006; Garaulet et al., 2007). AdipoR1 and AdipoR2 are abundantly expressed in many tissues and organs (Kadowaki \& Yamauchi, 2005; Dridi \& Taouis, 2009) with AdipoR1 predominantly expressed in skeletal muscle and AdipoR2 in liver (Yamauchi et al., 2003; Barb et al., 2007). Ohtani et al. (2011) demonstrated the existence of an autocrine-paracrine system of adiponectin in bovine mammary gland and the presence of adiponectin receptors was shown in human breast cancer cell line MCF-7 (Dieudonne et al., 2006). It is known that AdipoR1 knock-out mice are obese and glucose-intolerant and they have decreased energy expenditure. In contrast, AdipoR2 knock-out mice are lean, with decreased plasma cholesterol levels, and resistant to high-fat-diet induced obesity and show increased energy expenditure (Bjursell et al., 2007). This clearly demonstrates that both receptors are involved in energy metabolism, although they have opposing effects 
(Bjursell et al., 2007). According to Gonzalez et al. (2010) it is plausible to hypothesize that there is a different correlation between circulating adiponectin and AdipoR1 and AdipoR2 expressions. Moreover, AdipoR1 appears to mediate adiponectin effects via AMPK, whereas AdipoR2 via PPAR- $\alpha$ (Kadowaki et al., 2008). Furthermore, nutritional status, pregnancy and circulating leptin seem to control circulating adiponectin and AdipoR2 expression in tissue specific manner in rats, whereas AdipoR2 expression is not correlated with circulating adiponectin (Gonzalez et al., 2010).

The effects of adiponectin on liver and muscle tissue are well documented in human and mice (Yamauchi et al., 2001,2002, 2003; Berg et al., 2002; Kadowaki \& Yamauchi, 2005), but they have not been explored in other domestic animal models and dairy cows. It has been reported that adiponectin-deficient mice are insulin-resistant and present lower plasma insulin levels after glucose loading than wild-type mice (Kharroubi et al., 2003). Moreover, high circulating adiponectin levels were associated with a lower risk of development of type 2 diabetes and adiponectin was negatively correlated with circulating glucose (Hotta et al., 2000; Weyer et al., 2001; Spranger et al., 2003). The main impacts of adiponectin on glucose homeostasis and nutrient partitioning can be summarized as: (1) adiponectin decreases glucose output, increases FFA oxidation, and increases influx of NEFA in the liver; (2) adiponectin increases FFA utilization and stimulates glucose usage in muscle tissue. For these reasons, it is believed that adiponectin generally improves the ratio of glucose to FFA and consequently has insulin sensitizing activity (Kershaw \& Flier, 2004; Kadowaki \& Yamauchi, 2005; Garaulet et al., 2007; Shetty et al., 2009).

Generally, the mechanism of insulin sensitizing of adiponectin involves activation of 5' AMP-activated protein kinase (AMPK) and peroxisome proliferator-activated receptor alpha $(\operatorname{PPAR} \alpha)$, increased glucose uptake by GLUT4, increased glycolysis by phosphorylation of phosphofructokinase, and increased fatty acid oxidation by inactivation of acetyl CoA carboxylase and activation of carnitine palmitoyltransferase I (Yamauchi et al., 2001, 2002; Ahima, 2006; Kadowaki et al., 2006; Kamada et al., 2008). According to Combs et al. (2001) even a moderate surge in circulating adiponectin can suspend both the expression of hepatic gluconeogenic enzymes and the rate of endogenous glucose 
production. AMPK is a cellular energy sensor that is activated by a rise in the intracellular AMP/ ATP ratio. It is believed that adiponectin is a potent activator of AMPK and exerts its metabolic effects by activation of AMPK and subsequent deactivation of acetylcoenzyme A carboxylase (Yamauchi et al., 2002). This leads to decreased activation of malonyl CoA and increased activation of carnitine palmitoyltransferase I. Finally, activation of AMPK has been demonstrated to stimulate the recruitment of GLUT4 to the plasma membrane from cytoplasm, and to increase glucose uptake (Mullen et al., 2007).

The impact of adiponectin on metabolism in dairy cows is almost unknown and few studies have measured adiponectin in cows. Komatsu et al. (2007) assessed adiponectin mRNA change in adipose tissue, in peak-, late-, and non-lactating cows. This study demonstrated that adiponectin concentrations reached a peak in non-lactating cows, and this value was greater than both peak- and late-lactation adiponectin values. Raddatz et al. (2008) measured adiponectin concentrations in lactating Holstein cows for the first 11 weeks of lactation. Adiponectin concentrations increased from week 1 to week 4 postpartum and then declined to remain at $12-13 \mathrm{ng} / \mathrm{ml}$ for the remainder of the study. Circulating adiponectin did not correlate with BCS or energy corrected milk yield, and circulating adiponectin did not affect resumption of oestrous cycles. Puntenney (2006) examined the effect of prepartum dietary treatment, breed (Jersey versus Holstein), and BCS in dairy cows. None of these factors had an effect on circulating adiponectin. Circulating Adiponectin was not correlated with NEFA, insulin, glucose, tumour necrosis factor-alpha (TNF- $\alpha$ ), and BCS. Ohtani et al. (2011) examined mRNA expression levels of adiponectin and its receptors in various bovine tissues and mammary glands at different stages of lactation, and the effects of lactogenic hormones (insulin, dexamethasone and prolactin) and GH on mRNA expression of adiponectin receptors in cultured bovine mammary epithelial cells. AdipoR1 and AdipoR2 mRNAs were widely expressed in various bovine tissues, but adiponectin mRNA expression was significantly higher in adipose tissue than in other tissues. Although adiponectin mRNA expression was significantly decreased in the lactating mammary gland, AdipoR1 mRNA expression was significantly higher at peak lactation than at drying off. Moreover, in mammary epithelial cells lactogenic hormones and GH up-regulated AdipoR2 mRNA expression but did not change AdipoR1 mRNA. 
Lemor et al. (2009) reported that AdipoR1 and AdipoR2 mRNA abundance in adipose tissue decreased as high yielding cows moved from pregnancy to lactation.

Some evidence suggests that adiponectin could directly regulate reproductive functions. Adiponectin and its receptors are present in theca and granulosa cells, oocytes and the corpus luteum (Lord et al., 2005; Ledoux et al., 2006; Ramachandran et al., 2007; Chabrolle et al., 2007a, 2007b). Adiponectin is also present in porcine and human follicular fluid (Ledoux et al., 2006; Chabrolle et al., 2009). Adiponectin may influence steroidogenesis, but contradictory results have been reported (Ledoux et al., 2006; Lagaly et al., 2008; Chabrolle et al., 2009; Gutman et al., 2009; Pierre et al., 2009; Maillard et al., 2010). The response of theca cells to adiponectin may be regulated by LH and IGF-I. Supplementation with LH during the late follicular phase may enhance follicular insulin sensitivity, resulting in decreased androgen levels through a pathway mediated by increased production of adiponectin by the human follicle (Gutman et al., 2009). In the ovary, most of the adiponectin-induced modulations in gene and protein expression are mediated by AMPK (Chabrolle et al., 2007b) or ERK 1/2- mitogen-activated protein kinase (MAPK) dependent pathway (Maillard et al., 2010). In the ovary, the association between adiponectin and AMPK suggests that adiponectin may act as a key signal regulating the amount of energy required for growth of follicles and oocytes (Dupont et al., 2008). AdipoR1 or AdipoR2 knock-out mice are fertile, and thus adiponectin is not essential for normal ovarian function (Brochu-Gaudreau et al., 2010). It has been hypothesized that adiponectin effects on the ovary are mediated through its insulinsensitizing traits (Mitchell et al., 2005) and through its effect on IGF-I (Campos et al., 2007; Dupont et al., 2008; Michalakis \& Segars, 2010). Chappaz et al. (2008) concluded that when adiponectin was added to media culture, meiotic maturation and embryo development of porcine oocytes were influenced positively. This indicates an effect of adiponectin on early embryonic development, but it needs to be elucidated further.

Weyermann et al. (2006) and Martin et al. (2006) examined adiponectin and leptin in human milk. Both of these studies confirmed the presence of adiponectin in human milk. On the one hand, Weyermann et al. (2006) reported that concentrations of adiponectin and 
leptin varied strongly in maternal serum, cord blood, and breast milk, with only moderate correlations between both adipokines in maternal serum and breast milk. On the other hand, Martin et al. (2006) found that adiponectin concentrations in human milk are associated with stage of lactation, maternal adiposity (BMI), and ethnicity. In addition, Bronsky et al. (2006) measured adiponectin in human breast milk and found that milk adiponectin did not differ significantly in mothers who delivered boys versus girls. The authors also reported that there was a positive correlation between milk adiponectin and body weight of mothers before pregnancy, but no correlation between milk adiponectin and body weight at time of delivery.

Adiposity and circulating adiponectin are negatively correlated. According to Ishioka et al. (2006), canine adiponectin mRNA was detectable only in adipose tissue and obese dogs showed heavier body weights but lower circulating adiponectin. In the same study, circulating leptin was negatively correlated with circulating adiponectin. Kearns et al. (2006) reported that adiposity in horses was positively correlated with leptin and negatively correlated with adiponectin. Circulating adiponectin was negatively correlated with LWT, BMI, and insulin in humans (Berg et al., 2002; Matsuzawa et al., 2004; Ahima, 2006). No study has investigated the association of circulating adiponectin with LWT and BCS at calving in dairy cows. However, Raddatz et al. (2008) proposed that circulating adiponectin is not correlated with BCS in early lactating dairy cows.

Basu et al. (2009) investigated in utero the effect of gender dimorphism of adiponectin in humans. In this study it was reported that total adiponectin concentrations were higher in female compared with male foetuses. Furthermore, adiponectin was positively correlated with neonatal fat mass and percent body fat in female foetuses, and with lean mass in males. Many studies suggested higher circulating adiponectin in women compared to men (Kern et al., 2003; Silha et al., 2003; Tschritter et al., 2003), but no sexual dimorphism was noted between mares and geldings (Gordon et al., 2007). These findings implicate a complicacy of other factors, such as reproductive hormones, which possibly are involved in the regulation of adiponectin. Oestrogen was found to be negatively correlated with adiponectin in women, although pharmacologic doses given to women did not decrease 
adiponectin levels (Mazaki-Tovi et al., 2005). According to Barb et al. (2007) testosterone down-regulates adiponectin expression in adipose tissue, resulting in higher circulating adiponectin in females. The effect of testosterone on adiponectin is also unclear, with some studies reporting significant a suppressive effect on adiponectin and others reporting no effect (Mazaki-Tovi et al., 2005; Mitchell et al., 2005).

Dietary factors may modulate circulating adiponectin (Reis et al., 2010). High consumption of magnesium (Qi et al., 2005; Cassidy et al., 2009), caffeine (Williams et al., 2008), omega-3 polyunsaturated fatty acids (n-3 PUFA) (Duda et al., 2007), and dietary salt (Lely et al., 2007) were associated in humans with higher circulating adiponectin. According to Barnea et al. (2006) mice fed a High Fat (HF) diet exhibited significantly greater weight gain, abnormal oral glucose tolerance test curves, and elevated insulin resistance, although circulating adiponectin remained unchanged compared with controls. Jones et al. (2009) studied the effect of HF diet supplementation on a mouse model from a period around mating and throughout gestation. The HF diet increased maternal adiposity and circulating maternal leptin, and decreased serum adiponectin. Cassidy et al. (2009) observed that dietary intakes of carbohydrate, protein, and trans-fat were negatively associated with circulating adiponectin in human female twins. Pischon et al. (2005) showed that circulating adiponectin was negatively related to glycaemic load, but tended to be positively associated with total fat intake in men. Shimabukuro et al. (2007) studied the effects of consuming a high-carbohydrate meal, a HF meal, or a standard test meal on postprandial circulating adiponectin in healthy humans and found no association of circulating adiponectin with type of meal. Reis et al. (2010) reviewed the effects of diet components on adiponectin levels in rodent and human models. In this review it was concluded that diets rich in saturated fat reduce levels of adiponectin, whereas diets rich in PUFA and supplementation with n-3 PUFA increase both gene expression and circulating adiponectin. Gordon \& McKeever (2005) showed that circulating adiponectin in horses did not alter throughout a 24-hour blood sampling period and neither dextrose challenge nor oral grain challenge changed circulating adiponectin. The effects of dietary components on circulating adiponectin have not yet been demonstrated in ruminants. 
Many studies suggest an important role of adiponectin in regulation of insulin sensitivity, however, little is known about the regulatory role of this hormone during pregnancy (Mazaki-Tovi et al., 2005). Catalano et al. (2006) investigated the roles of adiponectin in regulating glucose and lipid metabolism in human pregnancy. The authors reported that circulating adiponectin was lower in the third trimester than in the pregravid condition and this hypoadiponectinaemia was reflected by a 2.5 -fold decrease in WAT adiponectin mRNA, and $25 \%$ increase in fat mass. In the same study, insulin infusion decreased high molecular weight adiponectin complexes in pregravid women and the suppressive effect of insulin on adiponectin was lost during pregnancy. Asai-Sato et al. (2006) studied the long term changes in circulating adiponectin during pregnancy and lactation along with its relations with prolactin in lean healthy women. They reported that circulating adiponectin declined slightly as pregnancy advanced and reached its lowest level during lactation. The authors also concluded that the lowest levels of adiponectin during lactation were possibly because prolactin influenced regulation of maternal metabolism by suppressing adiponectin. Prolactin suppresses circulating adiponectin in mice (Combs et al., 2003), but in a transgenic mouse model increased circulating adiponectin and prolactin levels were observed (Combs et al., 2004). Another study (Nien et al., 2007), conducted to investigate the effect of different levels of adiposity on circulating adiponectin in pregnant women, concluded that there was no difference in circulating adiponectin between pregnant and non-pregnant women and circulating adiponectin was negatively correlated with gestational age only among pregnant women of normal weight. Sir-Petermann et al. (2007) reported that circulating adiponectin was lower in women with gestational diabetes mellitus (GDM) than in women with normal glucose tolerance. Gao et al. (2008) looked for possible associations of TNF- $\alpha$, leptin, and adiponectin in mid-trimester women with gestational diabetes mellitus. Women with GDM have the highest circulating TNF- $\alpha$ and leptin and the lowest circulating adiponectin compared to those with gestational impaired glucose tolerance and to healthy controls at 14-20 weeks of gestation.

Adiponectin involvement in the control of glucose homeostasis in peripheral tissues via AdipoR1 and AdipoR2 receptors is beyond any doubt (Shetty et al., 2009; Garaulet et al., 2007; Giannessi et al., 2007). However, recent studies have implied a central regulatory 
role of adiponectin. Adiponectin intra-cerebro-ventricular (ICV) infusion in mice led to weight loss through increased energy expenditure (Qi et al., 2004). AdipoR1 and AdipoR2 were discovered to be expressed by neurons (including pro-opiomelanocortin (POMC) and neuropeptide Y (NPY) neurons) and astrocytes in the rat hypothalamic nuclei, and adiponectin presence was ascertained in cerebrospinal fluid (Steinberg \& Kemp, 2007). In addition, increased phosphorylation of AMPK in the rat hypothalamus was induced by adiponectin (Guillod-Maximin et al., 2009). It has been demonstrated that AMPK activation and deactivation in hypothalamic neural cells play a key role in monitoring energy status and regulating food intake (Andersson et al., 2004; Minokoshi et al., 2008). Leptin and adiponectin control AMPK, with leptin to deactivate (Minokoshi et al., 2008) and adiponectin to activate it (Kubota et al., 2007) in hypothalamic neurons. Kubota et al., 2007 showed that adiponectin binding to AdipoR1 in the murine arcuate hypothalamus enhanced AMPK activity and stimulated food intake. The discovery a role for adiponectin in stimulating appetite led Kubota et al. (2007) to propose the "fat-centric" hypothesis. According to this hypothesis, energy stores are maintained by the opposing central actions of leptin and adiponectin (Steinberg \& Kemp, 2007). However, this hypothesis needs to be further investigated.

Some studies have demonstrated multiple regulatory interactions between adiponectin and $\mathrm{GH}$ at central and peripheral level. Engström et al. (2003) reported that GH-deficient women who followed a nine-month treatment with exogenous GH supplementation showed elevated circulating adiponectin compared to controls. Lam et al. (2004) reported that circulating adiponectin was low in patients suffering from acromegaly, and circulating adiponectin increased after GH-lowering therapies. Fasshauer et al. (2004) demonstrated that GH was a putative regulator of AdipoR2 in 3T3-L1 adipocytes and that GH stimulated AdipoR2 synthesis was increased up to 4.8-fold during differentiation of 3T3-L1 preadipocytes. Moreover, this positive effect of GH on AdipoR2 expression could be reversed by GH withdrawal for 24 hours. Nilsson et al. (2005) demonstrated that gene expression of AdipoR1 and AdipoR2 in human adipose tissue is differentially regulated by prolactin and GH. Also, prolactin and GH reduced adiponectin secretion by human adipose tissue in vitro and in vivo in mice. Rodriguez-Pacheco et al. (2007) investigated the central 
role of adiponectin in regulating somatotroph and gonadotroph function. The authors showed that short-term adiponectin exposure abolished both basal and stimulated (by Ghrelin and GnRH) secretion of GH and LH by rat pituitary cells in vitro. Also, this study demonstrated the existence of a complete autocrine/ paracrine system of adiponectin in rat pituitary in which both adiponectin and its receptors are expressed. Thus, adiponectin seems to regulate energy expenditure, body reserves, and reproduction by playing a complicated central and peripheral role similar to leptin. Steyn et al. (2009) confirmed the regulatory effect of adiponectin on GH secretion in rat pituitary somatotrophs. However, this study demonstrated that adiponectin stimulated GH secretion in a dose-dependent manner through binding to either AdipoR1 or AdipoR2. Although these results are conflicting, they demonstrate interaction between adiponectin, GH, and their receptors at the central and peripheral level. The terms of this interaction needs to be further elucidated by both in vitro and in vivo experiments.

To conclude, adiponectin is a very interesting molecule but research has been mostly conducted in humans and rodents. There is only limited evidence for a role of adiponectin in nutrient partitioning, reproductive performance, and milk yield in dairy cattle. Thus, the interplay of adiponectin with other bovine hormonal and metabolic stimuli must be investigated further.

\subsection{Working hypotheses and objectives}

The literature review strongly suggested that reproductive performance of lactating dairy cows interacts with nutrition and BCS at calving and that the related hormonal and metabolic profile plays a role in cow fertility. This was the main hypothesis of the present thesis. This hypothesis has been developed in Chapter 3 in this $\mathrm{P} h \mathrm{D}$ project, and has been tested in a series of specific objectives. More specifically the objectives were to: 
- Examine the effect of two dietary treatments (high starch versus high fat diet) on metabolic profile and reproduction (Chapter 3).

- Explore the effect of BCS at calving on metabolic profile and reproductive performance (Chapter 3).

- Define optimum plasma insulin concentration in terms of reproduction, and address its impact on pregnancy rate and milk progesterone profile (Chapter 3).

The second hypothesis was that different BCS at calving, diets, milk yield potential and physiological stages will affect circulating and milk adiponectin values, and production traits, metabolic hormones and metabolites, and their relationships. This hypothesis has been developed in Chapter 4 in this $\mathrm{P} h \mathrm{D}$ project, and has been broken down into more specific objectives. More specifically the objectives were to:

- Measure circulating adiponectin and milk adiponectin in dairy cows (Chapter 4).

- Investigate the effects of diet and BCS at calving on circulating adiponectin (Chapter 4).

- Demonstrate the effect of stage of lactation on circulating adiponectin (Chapter 4).

- Investigate associations between leptin, adiponectin, production traits, and metabolic and hormonal signals (Chapter 4).

- Assess the association of circulating adiponectin with DMI in lactating dairy cows (Chapter 4).

The third hypothesis was that high yielding cows have lower circulating adiponectin than low yielding cows due to increased circulating GH and its antagonistic relationship with adiponectin. This hypothesis has been developed in Chapter 4 in this $\mathrm{P} h \mathrm{D}$ project.

The fourth hypothesis was that animals with elevated plasma adiponectin concentrations (up to three times) will express different hormonal, metabolic, productive, and/or reproductive pattern. This hypothesis has been developed in Chapter 5 in this $\mathrm{P} h \mathrm{D}$ project. 
The fifth hypothesis was that high yielding cows will regulate glucose homeostasis differently to low yielding cows, and glucose homeostasis in dairy cows will be regulated by adiponectin. This hypothesis has been developed in Chapter 5 in this $\mathrm{P} h \mathrm{D}$ project.

A general discussion summarizing and integrating the results of these experiments has been presented in Chapter 6 . 


\section{Effects of body condition score at calving and diet on circulating metabolic hormones, metabolites, and reproductive traits in dairy cows}

\subsection{INTRODUCTION}

Body condition score (BCS) is a rapid, non-invasive, inexpensive, but subjective way of assessing body reserves of the cow. It is easy applicable at the farm level and might give a more realistic view of the lipid and muscle reserves than live weight (Garnsworthy, 2006). In recent decades, BCS has been proved a useful management tool for assessing nutritional status and EB during lactation in dairy cows. Estimates of BCS are significantly correlated to subcutaneous fat (Garnsworthy \& Topps, 1982; Domecq et al., 1995; Heuer et al., 1999; Garnsworthy et al., 2006). Ruegg \& Milton (1995) reported that excessive loss of BCS during early lactation is related with metabolic diseases and Gearhart et al. (1990) described high BCS at parturition as a risk factor for reproductive performance in dairy cows. It is well known that body condition loss and especially BCS at certain points of the cow cycle impacts directly on DMI, NEB, fertility, milk yield and milk composition, and health of high yielding cow (Butler, 2003; Garnsworthy, 2006). According to Chagas et al. (2007) the perfect BCS fluctuation to minimize the effects of NEB on health and reproduction is BCS at calving 3.0-3.5 units with a nadir BCS of 2.5-3.0 units. Mulligan et al. (2006b) suggested BCS targets for drying-off point; 2.75 units, BCS at calving; 3.0 units, $\mathrm{BCS}$ at service $\geq 2.75$ units, and nadir BCS; 2.75 units.

According to Mulligan et al. (2006a, 2006b), over-fat cows at calving have a greater possibility of expressing fatty liver, ketosis, retained placenta, calving difficulties and milk fever. Butler \& Smith (1989) and Villa-Godoy et al. (1990) demonstrated that when NEB is accompanied by excess BCS loss then the result is declined fertility. Numerous studies showed the negative impact of excess or inadequate BCS at calving, BCS loss and nadir 
BCS on postpartum reproductive performance (Garnsworthy \& Topps, 1982; Heuer et al., 1999; Moreira et al., 2000; Yamada et al., 2003; Lopez-Gatius et al., 2003; Agenas et al., 2003; Roche et al., 2007b). The main outcome from these studies was that reproductive indices determining the reproductive efficiency of the herd, such as calving interval, days to first oestrus, days to first service, first service conception rate and number of services were related to $\mathrm{BCS}$, which is considered a key point of reproductive management.

BCS has been considered an indirect measure of nutritional status (Short et al., 1990; Garnsworthy, 2006; Bewley \& Schutz, 2008). That implies that BCS is affected by diet composition and DMI (Short et al., 1990; Garnsworthy, 2006). According to Short et al. (1990) the effect of nutrition on reproduction depends on whether nutritional differences exist before or after calving. In addition, BCS at calving is more important than postpartum BCS loss and consequently dietary and nutritional management of animals in the dry period is a crucial factor to ensure timely reproductive functionality after calving (Short et al., 1990).

The interaction between nutrition and reproduction is complicated and variable (Boland et al., 2001). This interaction involves both past and present nutritional status, but other factors such as genetic makeup, BCS, environmental influence, and physiological state are determinant modifiers of nutritional effects on reproductive performance (Short et al., 1990; Garnsworthy \& Webb, 1999; Lucy, 2001; Stevenson, 2001; Robinson et al., 2006). High fat diets with dietary total fat concentration over $50 \mathrm{~g} / \mathrm{kg}$ of DM depressed plasma insulin concentration in cows at the onset of the breeding period (Garnsworthy et al., 2008b). Conversely, high starch diets induced high plasma insulin concentration and increased the proportion of cows ovulating within 50 days of calving and reduced the interval from calving to first ovulation, and tended to reduce the intervals from calving to first service and to conception (Gong et al., 2002b). Moreover, Adamiak et al. (2005, 2006) in heifers (beef $\mathrm{X}$ dairy) and Fouladi-Nashta et al. (2005) in lactating dairy cows demonstrated that diets designed to increase plasma insulin concentration had negative effects on blastocyst rate in heifers and lactating cows. 
For the purposes of the present study, data generated by Garnsworthy et al. (2009) were reanalysed. Garnsworthy et al. (2009) provided evidence to support the hypothesis that pregnancy rate will be enhanced by feeding a diet that increased plasma insulin (HS diet) until cows resume ovarian cycles, and then switching to a diet (HF diet) that decreased plasma insulin during the mating period. In the study of Garnsworthy et al. (2009), the impact of BCS on reproductive performance of lactating cows was not assessed. Thus, the first objective of this study was to determine the effects of BCS at calving and diet on reproductive performance in early lactating dairy cows. It was hypothesized that different BCS at calving and diet would result in different metabolic profiles and reproductive performance. Hormonal and metabolic profiles of the cows were used to explain differences in reproductive traits such as days to first oestrus, pregnancy rate, and milk progesterone profile. Gong et al. (2002b) demonstrated that feeding a high starch diet to dairy cows for the first 50 days postpartum increased circulating insulin concentrations, and reproductive performance was enhanced. In addition, Fouladi-Nashta et al. (2007) reported that diets with a high fat content had beneficial effects on blastocyst rate in lactating dairy cows although they decreased circulating insulin. These two results taken together may imply that there is an optimum insulin concentration necessary for normal reproductive performance in lactating dairy cows. Moreover, it is known that fat cows at calving are more insulin resistant than thin cows at calving (Holtenius \& Holtenius, 2007; Sinclair, 2010). In the study of Garnsworthy et al. (2009) cows fed the high starch and high fat diets had normal to high concentrations of circulating insulin and expressed very low pregnancy rate $(26.7 \%)$. The second objective of the study was to test the hypothesis that there is an optimum insulin concentration necessary for normal reproductive performance in lactating dairy cows, and that is dependent on BCS at calving. 


\subsection{MATERIALS AND METHODS}

\subsubsection{Data}

This study utilized data generated by the study of Garnsworthy et al. (2009). Key points of materials and methods, further statistical analysis, and handle of the data are presented hereinafter.

\subsubsection{Experimental Design}

Thirty high-yielding multiparous Holstein dairy cows were blocked according to calving date and parity, and were allocated at random to two equal dietary treatment groups (HS and HF, 15 cows in each). Within dietary treatment groups, cows were selected on the basis of BCS, and divided into FAT and THIN cows. Cows with BCS at calving greater than 3.25 were the FAT group (19 cows in total; HS, 9 cows; HF, 10 cows ) and cows with BCS at calving equal or less than 3.25 were the THIN group (11 cows in total; HS, 6 cows; HF, 5 cows). Prepartum, all cows were fed on the same diet. Blood samples were taken from each cow from $2^{\text {nd }}$ week until $17^{\text {th }}$ week postcalving. The data were obtained from this experiment were panel, longitudinal or cross-sectional data. Cows were the panels, and dietary (DIET) and condition (CONDITION) treatments were nested within week of experiment (WEEK). Two diets were formulated to have equal concentrations of DM, ME and CP, but to differ in starch, fat and NDF concentrations (Table A.1 in appendix). Diet HS was expected to induce relatively high plasma insulin concentrations because of its higher starch and lower fat contents; Diet HF was expected to induce relatively low insulin concentrations because of its lower starch and higher fat contents. These diets were equivalent to the high and low insulin diets used by Gong et al. (2002), Fouladi-Nashta et al. (2005), and Garnsworthy et al. (2009). Milk progesterone was measured twice each week (either Monday and Thursday or Tuesday and Friday mornings) and a rise in progesterone was defined as above $3 \mathrm{ng} / \mathrm{ml}$ for two consecutive samples (Lamming \& Darwash, 1998; Garnsworthy et al., 2009). 


\subsubsection{Feeding and milking}

Cows were housed as one group in a freestall barn throughout the experiment, and were fed individually via electronic feeders (Roughage Intake Control feeders, Fullwood Ltd, Ellesmere, UK) that recorded feed intake automatically. Cows were milked by an automatic (robotic) milking system (AMS; Merlin, Fullwood Ltd.), which they entered voluntarily and were milked on average $2.65 \pm 0.09$ times per day. In order to encourage cows to use the AMS, $4 \mathrm{~kg}$ fresh weight of each cow's daily concentrate allocation was dispensed automatically in the AMS during milking (Garnsworthy et al., 2009).

\subsubsection{Reproductive management}

Cows were artificially inseminated at the first or second oestrus. Insemination was repeated at any subsequent oestrus until the end of the experiment at 120 days post partum. Oestrus was detected using a combination of behavioural observations, pedometer activity monitoring and milk progesterone profiles. Milk progesterone was monitored daily from 4 days before expected oestrus until signs of oestrus were detected; monitoring then returned to twice weekly until 4 days before the next expected oestrus (21 days later). Progesterone profiles were used subsequently to classify oestrous cycles as normal or abnormal (DOV1, DOV2, PCL1 or PCL2), following the definitions of Lamming \& Darwash (1998) (Table A.2 in appendix).

\subsubsection{Recording, sampling and analysis}

Milk yield and feed intake were recorded daily throughout the experiment. Live weight and BCS (units; 1 to 5) were recorded weekly. Milk samples were taken twice each week and analyzed for progesterone by ELISA (Ridgeway Scientific, Alvington, UK). Feed samples were taken weekly and pooled on a monthly basis for analysis of DM, CP, NDF, starch, sugars, fat and ME, as detailed in Garnsworthy et al. (2008b). Blood samples were taken every Wednesday at 09:30 $\mathrm{h}$ throughout of the sampling period for measurement of hormones and metabolites. Blood samples were analyzed for the following hormones: 
insulin, GH, IGF1, glucagon, and leptin, as detailed in Garnsworthy et al. (2009). Blood samples were analyzed for the following metabolites on a Bayer opera autoanalyzer (Bayer UK Ltd): urea-N, glucose, BOHB, and NEFA as detailed in Garnsworthy et al. (2009). All instances of ill health and veterinary treatments were recorded. The ultimate fate of each cow (subsequent calving or culling) was recorded to allow calculation of calving interval. For cows that were pregnant when culled, subsequent calving date was estimated as date of successful insemination plus 282 days; cows that were not pregnant when culled were omitted from statistical analysis of calving interval (Garnsworthy et al., 2009). 


\subsection{STATISTICAL ANALYSIS}

All data were analyzed using PASW ${ }^{\odot} 18$ Edition (SPSS Inc., Chicago, USA). Generalized Linear models (GLM) and Generalized Estimating Equations (GEE) were used to test the specific hypotheses (Lindsey, 1997; McCullagh \& Nelder, 1989; Dobson, 2002; Horton \& Lipsitz, 1999). Family distribution and link function were selected by running a null model including only the dependent variable and various combinations of family distribution and link function (Lindsey, 1997; McCullagh \& Nelder, 1989; Dobson, 2002; Horton \& Lipsitz, 1999). Models were compared in terms of values of Akaike's information criterion (AIC) criterion and the one with the lowest AIC value was chosen (Lindsey, 1997; McCullagh \& Nelder, 1989; Dobson, 2002; Horton \& Lipsitz, 1999). AIC is a statistical model fit index, defined as AIC $=-2 L_{m}+2 m$ where $L_{m}$ is the maximized log-likelihood and $m$ is the number of parameters in the model. Lower values of AIC indicate better fit of the model.

The biological validity of each model was tested at the post-prediction level and the appropriate model was selected by using AIC and the quasi-likelihood under the independence model criterion (QIC) for choosing the best correlation structure. QIC is adaptation of AIC for repeated measures, where quasi log likelihood is used instead of log likelihood of AIC for better model fit. As with AIC, smaller values of QIC indicate better model fit (Dobson, 2002). Moreover, the GEE models were selected based on this simple principle; working correlation matrix $(R$ matrix $)$ must at least in part reflect the real correlation structure of the data (Horton \& Lipsitz, 1999).

Missing or incomplete data are inherent in studies where repeated measurements are obtained from animals (because of ill health, culling, and funding limitation (planned missing data) some animals are excluded from the study) (Little \& Rubin, 1987; Allison, 2001; Frees, 2004) and this was the greatest limitation of the present study. On the one hand, exclusion of missing data from analysis may result in inconsistent parameter estimates and possible loss of statistical power (considerable reduction of the sample and estimation of unacceptably large standard errors) (Frees, 2004). Alternatively, inclusion of 
missing data in the analysis may lead to biased results (Allison, 2001). However, missing data in panel studies can be handled by the methods of maximum likelihood $(M L)$, restricted maximum likelihood (REML), and multiple imputation estimation (MI) (Allison, 2001; Schafer, 1997; Frees 2004). According to Allison (2001) ML estimation is proven to be an excellent method for handling panel missing data if the data are missing at random (MAR). According to Enders (2010) patterns of data missingness are not that important and $M L$ is well suited for any type of missing data. $M L$ was the method of choice to deal with the missing values in the present study. Common repeated measurements ANOVA (Albert, 1999; Davis, 2002) and Mixed Linear Models (Cnaan et al., 1997; Littell et al., 1998; Littell, 2002; Wang \& Goonewardene, 2004; Gueorguieva \& Krystal, 2004), which are alternatives for panel data analysis, were not the preferred method for analyzing the data, mainly because the data were nonparametric and unbalanced. Generalized linear models (GLM), generalized estimating equations (GEE), and log-linear Poisson models utilize $M L$ to calculate parameter estimates and were the choice for analyzing the data in the present study (McCullagh \& Nelder, 1989; Lindsey, 1997; Dobson, 2002). GEEs use quasi likelihood estimation and developed to extend the GLM to repeated measurements (Davis, 2002). GEE models were selected based on the principle that the working correlation ( $R$ matrix) must at least in part reflect the real correlation structure of the data (Dobson, 2002). Also, inclusion of robust estimator of covariance matrix in GEE models may provide more accurate parameter estimates (Zorn, 2000; Harden, 2011). Finally, GEE and GLM do not assume normality, but allow the choice of error distribution and link function that better fit the data, leading possibly to more efficient parameter estimates (McCullagh \& Nelder, 1989; Lindsey, 1997).

Models selected to test the effects of BCS at calving (CONDITION) and diet on reproductive and production traits and hormonal and metabolic profile are described in Tables 3.1 to 3.3 .

Milk progesterone profile variable (Normal; 16 cows, DOV1; 3 cows, DOV2; 5 cows, PCL2; 5 cows, and PCL1; 1 cow) was transformed to a binominal variable with two levels (NORMAL; 16 cows and ABNORMAL; 14 cows); pregnancy rate is a binomial variable 
with two categories (PREGNANT; 8 cows and NON PREGNANT; 22 cows). Milk progesterone profile and pregnancy rate were used as binomial response variables in Generalized linear models to ascertain if BCS at calving and diet affected them (Table 3.1).

The output of generalized logistic regression and common logistic regression models are presented as probabilities, unstandardized beta $(b)$ coefficients, and odds ratio (exp (b)) with 95\% confidence intervals (Peng et al., 2002). In common logistic regression models, Hosmer \& Lemeshow goodness of fit test and Likelihood Ratio test (LR) were carried out. The $L R$ test compared the selected model with the null model (a model which included only the intercept) and significant $P$ values $(P<0.05)$ indicated the superiority of the selected model. The Hosmer \& Lemeshow test was used to examine the fitness of selected models to the actual data and non-significant $P$ values $(P>0.05)$ indicated better fit of the model (Agresti, 1996; Hosmer \& Lemeshow, 2000).

Receiver Operating Characteristic (ROC) analysis was conducted to evaluate the performance and the accuracy of logistic regression models (Hand, 2010; Zou et al., 2007). ROC curve is the plot of model sensitivity (true positive) versus model 1-specificity (=false positive) ( $\mathrm{Liu} \& \mathrm{Li}, 2005)$. Area under the ROC curve $\left(\mathrm{AUC}_{R O C}\right)$, which is an overall statistic summary of model accuracy and ranges from zero to one, was calculated. $\mathrm{AUC}_{R O C}$ equals 0.5 when the ROC curve corresponds to random chance and 1 for perfect accuracy (Zou et al., 2007). According to Hosmer \& Lemeshow (2000) $\mathrm{AUC}_{R O C}=0.5$ corresponds to a model with bad performance and accuracy, whereas $0.7<\mathrm{AUC}_{R O C}<0.8$, $0.8<\mathrm{AUC}_{R O C}<0.9$, and $\mathrm{AUC}_{R O C} \geq 0.9$ are considered as acceptable, excellent, and outstanding performance and accuracy of the model, respectively. $\mathrm{AUC}_{R O C}$ is presented as mean $\pm \mathrm{SE}$.

Non-random associations between two categorical variables in $2 \mathrm{X} 2$ contingency tables (e.g. BCS at calving X Diet) were tested by Fisher's exact test. $P$ value equal or less than $0.05(P \leq 0.05)$ indicated statistically significant association between the two categorical variables. 
The effect of BCS at calving and diet on time from calving to pregnancy was examined by survival analysis (Klein \& Moeschberger, 2005; Selvin, 2008). Cows not pregnant at the end of the experiment were censored at 120 days postpartum and Kaplan-Meier estimates of the survivor function were compared for FAT and THIN cows, and cows fed high starch (HS) and high fat (HF) diets by using Log-rank test (Landau \& Everitt, 2004; Jenkins, 2005; Guo, 2010). The models selected for survival analysis are described in Table 3.4.

Parameter estimates of GEE models are presented as marginal means plus/minus standard error of the difference (SED) in tables. Estimated marginal means, SEs and SEDs were obtained by using Least Significant Difference ( $L S D)$ adjustment for multiple comparisons in $\mathrm{PASW}^{\odot} 18$ statistical program. $P$ values were calculated by Newton-Raphson Maximum Likelihood $(M L)$ method and effects were considered statistically significant when $P$ value was less than $0.05(P<0.05)$. 
Table 3.1: Selected models for analysis of reproductive traits

\begin{tabular}{|c|c|c|c|c|c|c|c|}
\hline $\begin{array}{l}\text { REPRODUCTIVE TRAIT } \\
\text { (RESPONSE VARIABLE: Y) }\end{array}$ & $\begin{array}{l}\text { TYPE OF } \\
\text { MODEL }\end{array}$ & $\begin{array}{l}\text { PREDICTORS } \\
(X)\end{array}$ & $\begin{array}{l}\text { CONTINUOUS } \\
\text { COVARIATES }\end{array}$ & $\begin{array}{c}\text { ERROR } \\
\text { DISTRIBUTION } \\
\text { FUNCTION }\end{array}$ & $\begin{array}{l}\text { LINK } \\
\text { FUNCTION }\end{array}$ & $\begin{array}{l}\text { COVARIANCE } \\
\text { MATRIX }\end{array}$ & STATISTICS \\
\hline $\begin{array}{l}\text { - Milk P4 Profile (binomial } \\
\text { variable with two levels; } \\
0=\text { Normal } \\
1=\text { Abnormal) }\end{array}$ & $\begin{array}{l}\text { Multiple } \\
\text { Logistic } \\
\text { regression }\end{array}$ & $\begin{array}{l}- \text { CONDITION } \\
\text {-DIET }\end{array}$ & $\begin{array}{l}\text { Insulin } \\
\text { IGF-I } \\
\text { Glucose } \\
\text { Parity }\end{array}$ & $\begin{array}{l}\text { Bernoulli } \\
\text { (Binomial) }\end{array}$ & Logit & Robust & $\begin{array}{l}\mathrm{n}=29 ; \mathrm{AIC}=35 ; \quad L R\left(\chi^{2}=13.79,\right. \\
d f=6, P=0.032)\end{array}$ \\
\hline $\begin{array}{l}\text {-Milk P4 Profile (binomial } \\
\text { variable with two levels; } \\
0=\text { Normal } \\
1=\text { Abnormal) }\end{array}$ & GLM & $\begin{array}{l}- \text { CONDITION } \\
- \text { DIET }\end{array}$ & PARITY & $\begin{array}{l}\text { Bernoulli } \\
\text { (Binomial) }\end{array}$ & Logit & Robust & $\begin{array}{l}\mathrm{n}=30 ; \mathrm{AIC}=36 ; L R\left(\chi^{2}=10\right. \\
d f=3, P=0.018)\end{array}$ \\
\hline $\begin{array}{l}\text {-Pregnancy (binomial } \\
\text { variable with two levels; } 0= \\
\text { non pregnant } \\
1=\text { pregnant) }\end{array}$ & GLM & $\begin{array}{l}- \text { CONDITION } \\
\text {-DIET }\end{array}$ & PARITY & $\begin{array}{l}\text { Bernoulli } \\
\text { (Binomial) }\end{array}$ & Logit & Robust & $\begin{array}{l}\mathrm{n}=30 ; \mathrm{AIC}=35 ; L R\left(\chi^{2}=6.2,\right. \\
d f=2, P=0.045)\end{array}$ \\
\hline $\begin{array}{l}\text { - Pregnancy (binomial } \\
\text { variable with two levels; } 0= \\
\text { non pregnant } \\
1=\text { pregnant) }\end{array}$ & GLM & $\begin{array}{l}\bullet \text { MILK P4 } \\
\text { PROFILE }\end{array}$ & - & $\begin{array}{l}\text { Bernoulli } \\
\text { (Binomial) }\end{array}$ & Logit & Robust & $\begin{array}{l}\mathrm{n}=30 ; \mathrm{AIC}=33 ; \quad L R\left(\chi^{2}=3.89, d f=1,\right. \\
P=0.048)\end{array}$ \\
\hline $\begin{array}{l}\text {-Pregnancy (binomial } \\
\text { variable with two levels; } 0= \\
\text { non pregnant } \\
1=\text { pregnant) }\end{array}$ & $\begin{array}{l}\text { Multiple } \\
\text { Logistic } \\
\text { regression }\end{array}$ & $\begin{array}{l}\bullet \text { CONDITION } \\
\bullet \text { DIET }\end{array}$ & $\begin{array}{l}\text { Insulin } \\
\text { PARITY }\end{array}$ & $\begin{array}{l}\text { Bernoulli } \\
\text { (Binomial) }\end{array}$ & Logit & Robust & $\begin{array}{l}\mathrm{n}=30 ; \mathrm{AIC}=33 ; \quad L R\left(\chi^{2}=16.56\right. \\
d f=4, P=0.002)\end{array}$ \\
\hline -Days to Oestrus & GLM & $\begin{array}{l}-C O N D I T I O N \\
\bullet \text { DIET }\end{array}$ & PARITY & Poisson & $\log$ & Model Based & $\begin{array}{l}\mathrm{n}=28 ; \quad \mathrm{AIC}=267 ; L R\left(\chi^{2}=4, d f=3\right. \\
P=0.26)\end{array}$ \\
\hline
\end{tabular}


Table 3.2: Selected models for analysis of plasma metabolic hormones and metabolites

\begin{tabular}{|c|c|c|c|c|c|c|c|c|}
\hline $\begin{array}{c}\text { Hormone } \\
\text { (RESPONSE } \\
\text { VARIABLE: } Y \text { ) }\end{array}$ & $\begin{array}{l}\text { TYPE OF } \\
\text { MODEL }\end{array}$ & $\begin{array}{l}\text { PREDICTORS } \\
(X)\end{array}$ & $\begin{array}{l}\text { CONTINUOUS } \\
\text { COVARIATES }\end{array}$ & $\begin{array}{c}\text { ERROR } \\
\text { DISTRIBUTION } \\
\text { FUNCTION }\end{array}$ & $\begin{array}{c}\text { LINK } \\
\text { FUNCTION }\end{array}$ & $\begin{array}{l}\text { COVARIANCE } \\
\text { MATRIX }\end{array}$ & $\begin{array}{c}\text { WORKING } \\
\text { CORRELATION } \\
\text { MATRIX }\end{array}$ & STATISTICS \\
\hline $\begin{array}{l}\text { - Insulin } \\
\text { - Leptin } \\
\text {-IGF-I } \\
\text { - Glucagon }\end{array}$ & GEE & $\begin{array}{l}\text {-CONDITION } \\
\text {-DIET } \\
\text {-WEEK }\end{array}$ & PARITY & $\begin{array}{l}\text { Gaussian } \\
\text { (Normal) }\end{array}$ & Identity (ID) & Robust & Exchangeable & $\begin{array}{l}n=427 ; Q \mid C=66 \\
n=424 ; Q \mid C=395 \\
n=224 ; Q \mid C=424,623 \\
n=234 ; Q \mid C=116,230\end{array}$ \\
\hline$\bullet$ GH & $G E E$ & $\begin{array}{l}\text {-CONDITION } \\
\text {-DIET } \\
\text {-WEEK }\end{array}$ & PARITY & Inverse Gaussian & $\log$ & Robust & Exchangeable & $\mathrm{n}=222 ; \mathrm{QIC}=67$ \\
\hline $\begin{array}{l}\text { - Glucose } \\
\text {-Urea }\end{array}$ & GEE & $\begin{array}{l}\text {-CONDITION } \\
\text {-DIET } \\
\text {-WEEK }\end{array}$ & PARITY & $\begin{array}{l}\text { Gaussian } \\
\text { (Normal) }\end{array}$ & Identity (ID) & Robust & Exchangeable & $\begin{array}{l}n=225 ; Q I C=100 \\
n=231 ; Q \mid C=131\end{array}$ \\
\hline $\begin{array}{l}\bullet B O H B \\
\text {-NEFA }\end{array}$ & GEE & $\begin{array}{l}\text {-CONDITION } \\
\text {-DIET } \\
\text {-WEEK }\end{array}$ & PARITY & Inverse Gaussian & $\log$ & Robust & Exchangeable & $\begin{array}{l}n=233 ; Q I C=134 \\
n=233 ; Q I C=204\end{array}$ \\
\hline
\end{tabular}


Table 3.3: Selected models for analysis of production traits

\begin{tabular}{|c|c|c|c|c|c|c|c|c|}
\hline $\begin{array}{l}\text { PRODUCTIVE } \\
\text { TRAIT } \\
\text { (RESPONSE } \\
\text { VARIABLE: } Y \text { ) }\end{array}$ & $\begin{array}{l}\text { TYPE OF } \\
\text { MODEL }\end{array}$ & $\begin{array}{l}\text { PREDICTORS } \\
(X)\end{array}$ & $\begin{array}{l}\text { CONTINUOUS } \\
\text { COVARIATES }\end{array}$ & $\begin{array}{l}\text { ERROR } \\
\text { DISTRIBUTION } \\
\text { FUNCTION }\end{array}$ & $\begin{array}{c}\text { LINK } \\
\text { FUNCTION }\end{array}$ & $\begin{array}{l}\text { COVARIANCE } \\
\text { MATRIX }\end{array}$ & $\begin{array}{l}\text { WORKING } \\
\text { CORRELATION } \\
\text { MATRIX }\end{array}$ & STATISTICS \\
\hline $\begin{array}{l}\bullet \text { DMI } \\
\bullet \text { Milk Yield } \\
\bullet \text { LWT } \\
\bullet \text { BCS }\end{array}$ & GEE & $\begin{array}{l}- \text { CONDITION } \\
\text {-DIET } \\
\text {-WEEK }\end{array}$ & PARITY & $\begin{array}{l}\text { Gaussian } \\
\text { (Normal) }\end{array}$ & Identity (ID) & Robust & Exchangeable & $\begin{array}{l}n=460 ; Q I C=4,306 \\
n=460 ; Q \mid C=29,954 \\
n=460 ; Q I C=271,156 \\
n=473 ; Q I C=135\end{array}$ \\
\hline $\begin{array}{l}\text {-Nadir BCS } \\
\text {-Nadir Week } \\
\text { - } \triangle \mathrm{BCS}\end{array}$ & GLM & $\begin{array}{l}- \text { CONDITION } \\
\bullet \text { DIET }\end{array}$ & PARITY & $\begin{array}{l}\text { Gaussian } \\
\text { (Normal) }\end{array}$ & Identity (ID) & Robust & -- & $\begin{array}{l}\mathrm{n}=30 ; \mathrm{AIC}=24 ; \quad L R\left(\chi^{2}=6.4, d f=3, P=0.09\right) \\
\mathrm{n}=30 ; \mathrm{AIC}=24 ; \quad L R\left(\chi^{2}=10.2, d f=3, P=0.017\right) \\
\mathrm{n}=30 ; \mathrm{AIC}=42 ; \quad L R\left(\chi^{2}=12, d f=3, P=0.01\right)\end{array}$ \\
\hline
\end{tabular}

Table 3.4: Selected models for survival analysis (Kaplan- Meier models) of interval from calving to conception

\begin{tabular}{|c|c|c|c|c|c|c|c|}
\hline $\begin{array}{l}\text { TIME } \\
\text { VARIABLE } \\
\text { (Survival time) }\end{array}$ & $\begin{array}{l}\text { TYPE OF } \\
\text { MODEL }\end{array}$ & $\begin{array}{l}\text { STATUS } \\
\text { VARIABLE (Event } \\
\text { variable) }\end{array}$ & $\begin{array}{l}\text { FACTORS } \\
\text { (Group variables) }\end{array}$ & $\begin{array}{l}\text { SURVIVAL } \\
\text { FUNCTIONS } \\
\text { COMPARISON TEST }\end{array}$ & OBSERVATIONS & $\begin{array}{l}\text { RIGHT CENSORED } \\
\text { OBSERVATIONS }\end{array}$ & $\begin{array}{l}\text { UNCENSORED } \\
\text { OBSERVATIONS }\end{array}$ \\
\hline $\begin{array}{l}\text { Days post } \\
\text { partum } \\
(0-120)\end{array}$ & $\begin{array}{l}\text { Kaplan- } \\
\text { Meier }\end{array}$ & $\begin{array}{c}\text { Pregnancy } \\
\text { (binomial variable } \\
\text { with two levels; } \\
0=\text { non pregnant } \\
1=\text { event= pregnant) }\end{array}$ & $\begin{array}{l}\text {-CONDITION } \\
\text {-DIET }\end{array}$ & $\begin{array}{c}\text { Log-Rank } \\
\text { (Mantel-Cox) }\end{array}$ & 30 & 22 & 8 \\
\hline
\end{tabular}




\subsection{RESULTS}

\subsubsection{Consistency of BCS at calving tretments}

There was no association between BCS at calving and diet, and thus the retrospective classification of cows as FAT and THIN was not biased by the original dietary treatments (Fisher's exact test, $\mathrm{n}=30, \mathrm{P}=1.0$, two-sided) (Table 3.5).

\subsubsection{Effect of milk progesterone profile on pregnancy rate}

Cows with NORMAL milk progesterone profile had higher probability to be pregnant ( $44 \%$ versus $\left.7 \%, \mathrm{AUC}_{R O C}=0.74 \pm 0.08\right)$ than cows with $\mathrm{ABNORMAL}$ milk progesterone profile $(P<0.05)$ (Table 3.6).

\subsubsection{Effects of BCS at calving and diet on reproductive performance}

THIN cows at calving had higher probability to be pregnant (52\% versus $14 \%$, $\left.\mathrm{AUC}_{R O C}=0.79 \pm 0.09\right)$ than FAT cows at calving $(P<0.05)$ (Table 3.7). THIN cows at calving had lower probability to express ABNORMAL milk progesterone profile $(18 \%$ versus $\left.62 \%, \mathrm{AUC}_{R O C}=0.85 \pm 0.07\right)$ than $\mathrm{FAT}$ cows at calving $(P<0.05)$ (Table 3.7). There was no effect of diet on probability of cows to be pregnant within 120 days postpartum (Table 3.9). Cows fed the HS diet had higher probability to express ABNORMAL milk progesterone profile ( $65 \%$ versus $29 \%, \mathrm{AUC}_{R O C}=0.85 \pm 0.07$ ) than cows fed the HF diet $(P<0.05)$ (Table 3.8). There was no effect of BCS at calving and diet on days to first oestrus (Table 3.9). Survival analysis showed that FAT cows had a shorter interval from calving to conception than THIN cows, but FAT cows had a lower probability to be pregnant within 120 days postpartum than THIN cows $(P<0.05)$ (Figure 3.1). However, survival analysis demonstrated that there was no effect of diet on probability of cows to be 
pregnant within 120 days postpartum and cows fed the HS diet did not have significantly different interval from calving to conception compared to cows fed the HS diet (Figure 3.2).

\subsubsection{Effects of BCS at calving and diet on circulating metabolic hormones}

THIN cows at calving had higher circulating IGF-I $(147.5 \pm 10.12 \mathrm{ng} / \mathrm{ml}$ versus $104.9 \pm 9.30$ $n g / m l)$ than FAT cows at calving $(P<0.05)$ (Table 3.10). There was no effect of BCS at calving on circulating insulin, leptin, glucagon, and GH (Table 3.10). Cows fed the HS diet had higher circulating insulin $(0.49 \pm 0.03 \mathrm{ng} / \mathrm{ml}$ versus $0.41 \pm 0.03 \mathrm{ng} / \mathrm{ml})$ than cows fed the HF diet $(P<0.05)$ (Table 3.11). There was no effect of diet on circulating IGF-I, leptin, glucagon, and GH (Table 3.11).

\subsubsection{Effects of BCS at calving and diet on circulating metabolites}

THIN cows at calving had lower circulating NEFA $(0.32 \pm 0.03 \mathrm{mmol} / \mathrm{l}$ versus $0.41 \pm 0.03$ $\mathrm{mmol} / \mathrm{l})$ than FAT cows at calving $(P<0.05)$ (Table 3.12). There was no effect of BCS at calving on circulating glucose, BOHB, and urea (Table 3.12). Cows fed the HS diet had higher circulating urea $(2.83 \pm 0.14 \mathrm{mmol} / \mathrm{l}$ versus $2.32 \pm 0.14 \mathrm{mmol} / \mathrm{l})$ than cows fed the HF diet $(P<0.05)$ (Table 3.13). Circulating BOHB was lower $(P<0.05)$ for cows fed the HS diet $(0.485 \pm 0.040 \mathrm{mmol} / \mathrm{l})$ than for cows fed the HF diet $(0.650 \pm 0.040 \mathrm{mmol} / \mathrm{l})$ (Table 3.13). There was no effect of diet on circulating glucose and NEFA (Table 3.13).

\subsubsection{Effects of BCS at calving and diet on production traits}

THIN cows at calving had lower milk yield $(40.5 \pm 2.5 \mathrm{~kg} / \mathrm{d}$ versus $47.7 \pm 1.6 \mathrm{~kg} / \mathrm{d})$ than FAT cows at calving $(P<0.05)$ (Table 3.15). There was no effect of BCS at calving on LWT and DMI (Table 3.14). THIN cows at calving had lower BCS (2.36 \pm 0.06 units versus 
$2.66 \pm 0.08$ units), $\triangle \mathrm{BCS}(0.47 \pm 0.11$ units versus $0.82 \pm 0.09$ units $)$ and nadir BCS ( $2.02 \pm 0.06$ units versus $2.30 \pm 0.09$ units), and shorter nadir week ( $4.5 \pm 0.8$ weeks versus $6.5 \pm 0.8$ weeks) than FAT cows at calving $(P<0.05)$ (Table 3.14).

There was no effect of diet on LWT, DMI, milk yield, BCS, $\triangle \mathrm{BCS}$, nadir week, and nadir BCS (Table 3.15).

\subsubsection{Effect of insulin on pregnancy rate}

Multiple logistic regression analysis examined the effects of insulin, BCS at calving, diet, and parity on pregnancy rate. Insulin had a strong negative effect $\left(O R=1.11 * 10^{-07} \sim 0,95 \%\right.$ CI for $O R(0,0.03), b=-16.01)$ on the odds of cows to be pregnant $(P=0.012)$, holding the other predictors of the model at a fixed value. Dietary treatments $(O R=0.73,95 \% \mathrm{CI}$ for $O R(0.10,5.33), \mathrm{b}=-0.31)$ had no effect on the odds of cows to be pregnant $(P=0.76)$. BCS at calving (categorical variable with two levels; $0=\mathrm{FAT}, 1=\mathrm{THIN})(O R=52.32,95 \% \mathrm{CI}$ for $O R(5.79,472.38), b=3.96, P=0.001)$ and parity $(O R=0.21,95 \% \mathrm{CI}$ for $O R(0.05,0.85)$, $b=-1.54, P=0.03$ ) had a negative effect on the odds of cows to be pregnant, holding the other predictors of the model at a fixed value (Table 3.16).

The output of this model was expressed as probability of cows to be pregnant. The negative association of circulating insulin concentrations with the probability of cows to be pregnant is illustrated in Figure 3.3. This graph presents that the optimum insulin concentration that maximized the probability of cows to be pregnant ranged from 0.2 to 0.3 $\mathrm{ng} / \mathrm{ml}$, whereas insulin greater than $0.6 \mathrm{ng} / \mathrm{ml}$ tended to zero the probability of cows to be pregnant. Probability of cows to be pregnant was adjusted for different condition status at calving (FAT versus THIN), dietary treatments (HS versus HF), and circulating insulin concentrations (Figure 3.4, Figure 3.5, and Figure 3.6). According to Figure 3.4, THIN cows at calving had higher probability to be pregnant than FAT cows at calving $(P<0.05)$, when insulin ranged from 0.3 to $0.6 \mathrm{ng} / \mathrm{ml}$. Moreover, there was no effect of diet on probability of cows to be pregnant $(P>0.05)$, when the animals had approximately the same 
insulin concentration and insulin concentration ranged from 0.1 to $1.2 \mathrm{ng} / \mathrm{ml}$ (Figure 3.5). Also, there was no effect of (BCS at calving*diet) on probability of cows to be pregnant $(P>0.05)$ (Figure 3.6).

\subsubsection{Effects of insulin, IGF-I, glucose, and parity on milk progesterone profile}

Multiple logistic regression analysis examined the effects of insulin, IGF-I, glucose, and parity on milk progesterone profile. Each exponentiated coefficient in this model is the change in odds for a unit increase in the corresponding predictor variable holding other variables at certain value. Odds ratio $(O R)$ for insulin was very high and that means strong positive effect of insulin on the odds for cows to express abnormal milk progesterone profile $(P<0.05)$ (Table 3.17). OR for circulating glucose was very low and that means strong negative effect of circulating glucose on the odds for cows to express abnormal milk progesterone profile $(P<0.05)$ (Table 3.5). OR for parity was $8.59(P<0.05)$ (Table 3.17). So holding insulin, IGF-I, BCS at calving, diet, and glucose at a fixed value, an increase of parity by 1 will increase the odds for cows to express atypical milk progesterone profile by $759 \%$. IGF-I $(O R=0.97)$ also tended to have negative influence on the odds for cows to express abnormal milk progesterone profile $(P \leq 0.1)$, holding the other predictors of the model at a fixed value (Table 3.17).

The output of this model was expressed as probability for cows to express abnormal milk progesterone profile. The positive association of circulating insulin concentrations with the probability for cows to express abnormal milk progesterone profile is illustrated in Figure 3.7. This graph presents that the optimum insulin concentration that minimized the probability for cows to express abnormal milk progesterone profile ranged from 0.1 to 0.3 $\mathrm{ng} / \mathrm{ml}$, whereas insulin greater than 0.5 to $0.6 \mathrm{ng} / \mathrm{ml}$ tended to maximize the probability for cows to express abnormal milk progesterone profile. Probability for cows to express atypical milk progesterone profile was adjusted for different condition status at calving (FAT versus THIN), dietary treatments (HS versus HF), and circulating insulin concentrations (Figure 3.8, Figure 3.9, and Figure 3.10). According to Figure 3.8, THIN 
cows at calving had lower probability to to express abnormal milk progesterone profile than FAT cows at calving $(P<0.05)$, when the animals had approximately the same insulin concentration and insulin concentration ranged from 0.4 to $0.9 \mathrm{ng} / \mathrm{ml}$. Moreover, there was no effect of diet on probability for cows to express abnormal milk progesterone profile $(P>0.05)$, when the animals had approximately the same insulin concentration and insulin concentration ranged from 0.1 to $1.2 \mathrm{ng} / \mathrm{ml}$ (Figure 3.9). However, THIN cows at calving that fed the HF diet had lower probability to express abnormal milk progesterone profile than THIN cows at calving that fed the HS diet $(P<0.05)$, when the animals had approximately the same insulin concentration and insulin concentration ranged from 0.1 to $0.6 \mathrm{ng} / \mathrm{ml}$. (Figure 3.10). In addition, THIN cows at calving that fed the HF diet had lower probability to express abnormal milk progesterone profile than THIN cows at calving that fed the HS diet $(P<0.05)$, when insulin concentration ranged from 0.1 to $0.3 \mathrm{ng} / \mathrm{ml}$. 
Table 3.5: Crosstabulation of BCS at calving with diet. Association between BCS at calving and diet was tested by performing Fisher's exact test $(\mathrm{n}=30, P=1.0$, two-sided $)$. There was no association between BCS at calving and diet.

\begin{tabular}{cccc}
\hline \hline & BCS at calving & & Total \\
\hline Diet & FAT & THIN & 6 \\
HS & 9 & 5 & 15 \\
HF & 10 & 11 & 30 \\
Total & 19 & & 15 \\
\hline \hline
\end{tabular}


Table 3.6: Effect of milk progesterone profile on probability cows to be pregnant

\begin{tabular}{|c|c|c|c|c|c|}
\hline \multirow{2}{*}{$\begin{array}{c}\text { Treatment: } \\
\text { Parameters: }\end{array}$} & \multicolumn{4}{|c|}{ MILK PROGESTERONE PROFILE } & \multirow[b]{2}{*}{$\boldsymbol{P}$} \\
\hline & NORMAL ${ }^{\dagger}$ & $n_{1}^{\partial}$ & ABNORMAL $^{\dagger}$ & $n_{2}{ }^{\partial}$ & \\
\hline Probability for cows to be pregnant & $0.44(0.22,0.68)$ & 15 & $0.07(0,0.20)$ & 15 & 0.049 \\
\hline \multicolumn{6}{|c|}{$\begin{array}{l}{ }_{0}^{+} \text {Columns are means with } 95 \% \text { Confidence Interval (CI). } \\
n_{1} \text { and } n_{2} \text { are the numbers of animals expressed NORNAL and ABNORMAL milk progesterone profile, respectively. }\end{array}$} \\
\hline Treatment: & \multicolumn{4}{|c|}{ CONDITION } & \\
\hline Parameters: & FAT $^{\dagger}$ & $n_{1}{ }^{2}$ & THIN $^{\dagger}$ & $n_{2}{ }^{\partial}$ & $\boldsymbol{P}$ \\
\hline Probability for cows to be pregnant & $0.14(0,0.20)$ & 15 & $0.52(0.24,0.81)$ & 15 & 0.017 \\
\hline $\begin{array}{l}\text { Probability for cows to express } \\
\text { abnormal milk progesterone profile }\end{array}$ & $0.62(0.43,0.80)$ & 15 & $0.18(0,0.38)$ & 15 & 0.02 \\
\hline
\end{tabular}

Columns are means with $95 \%$ Confidence Interval (CI).

${ }^{\partial} n_{1}$ and $n_{2}$ are the numbers of animals assessed FAT and THIN at calving, respectively. 


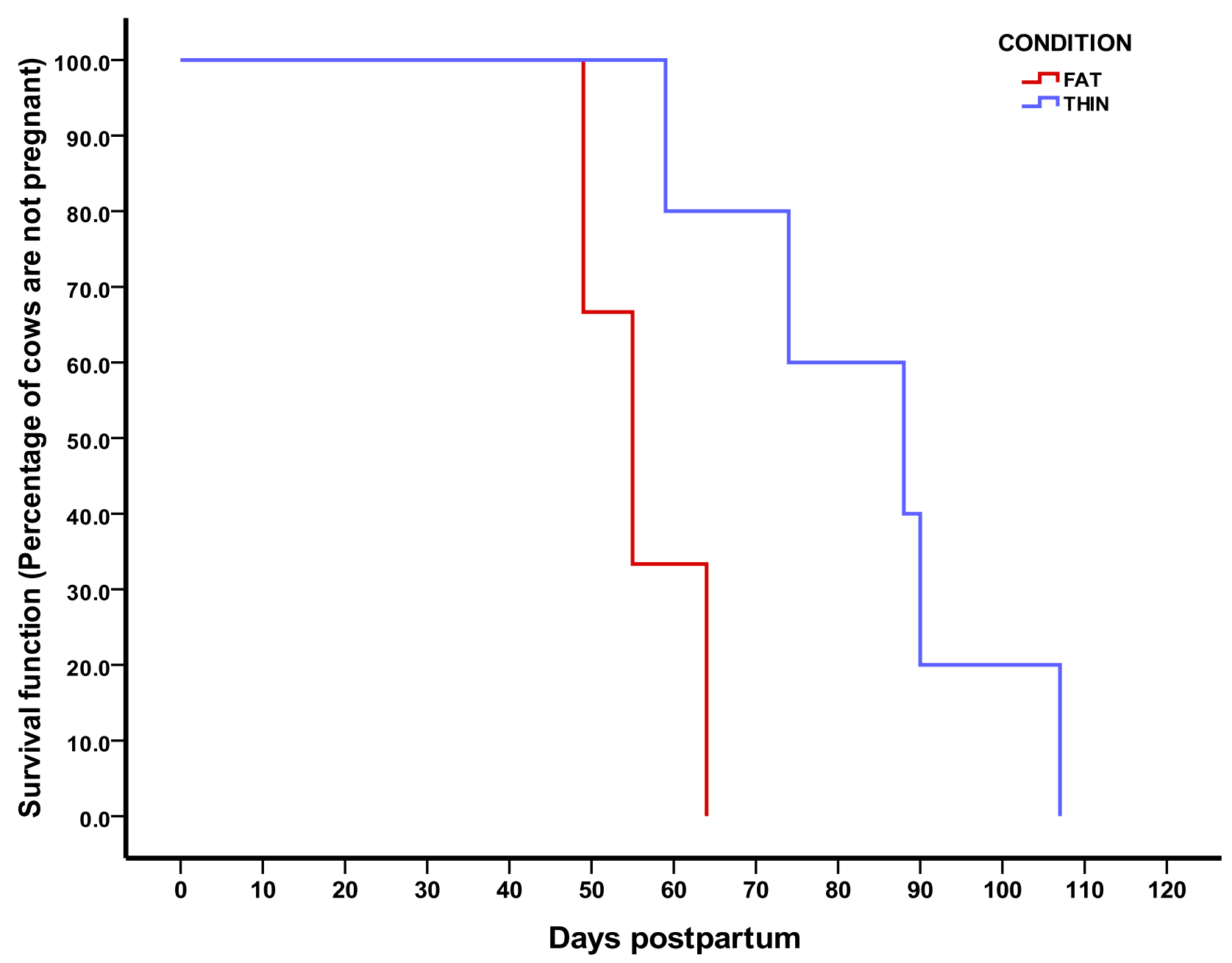

Figure 3.1: Calving-to-conception survival analysis curves for FAT and THIN cows at calving. The survival curve for THIN cows is higher than the curve for FAT cows $(n=30$, Log-rank test $P=0.022)$. Vertical drop in the survival curves indicates an event (a cow became pregnant). FAT cows at calving had a shorter interval from calving to conception ( $55 \pm 4.9$ days postpartum versus $88 \pm 15.3$ days postpartum) than THIN cows at calving. 


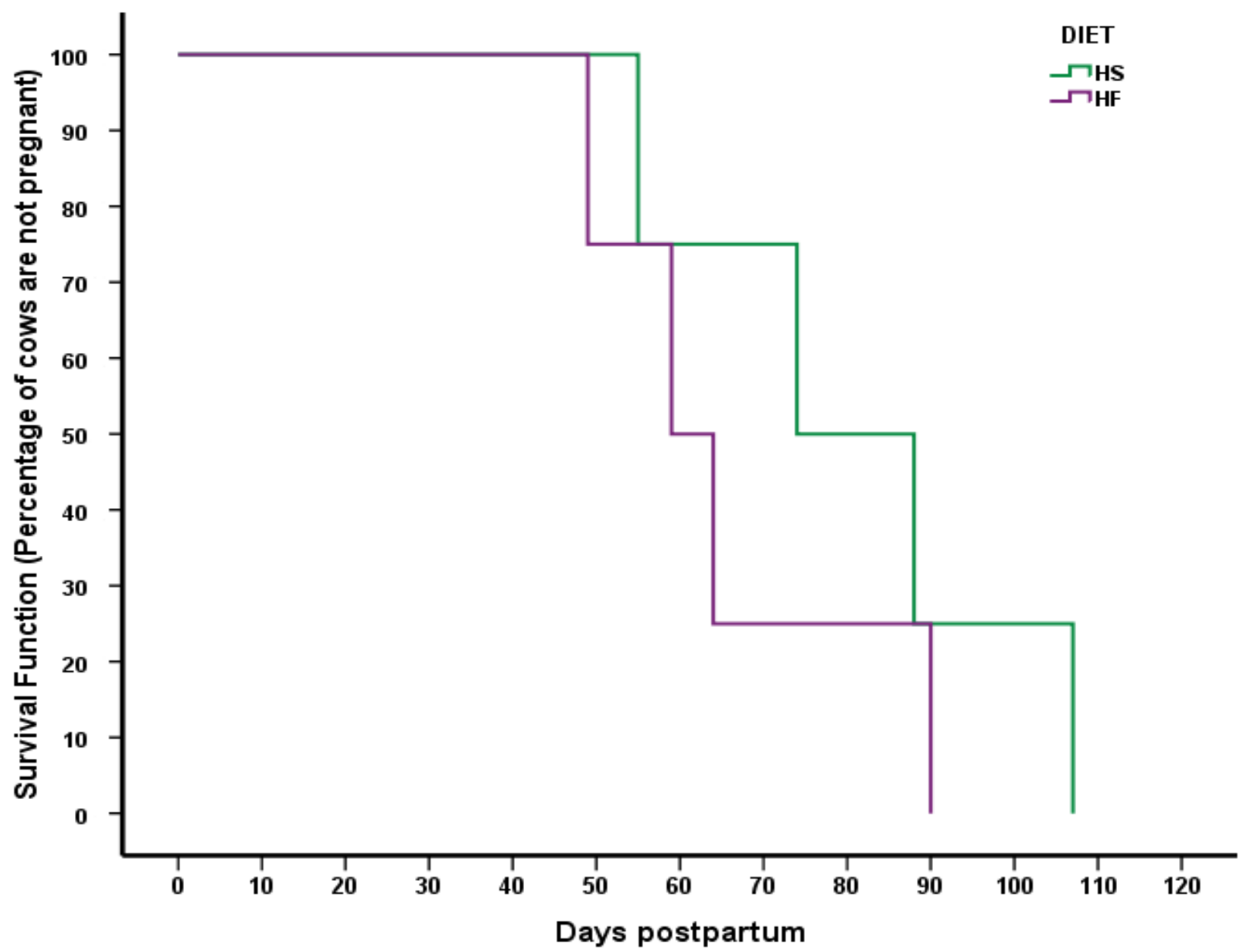

Figure 3.2: Calving-to-conception survival analysis curves for cows fed the high starch (HS) and the high fat (HF) diet. There are no differences in the survival curves for cows fed with HS and HF diet $(n=30$, Log-rank test $P=0.40)$. Vertical drop in the survival curves indicates an event (a cow became pregnant). Cows fed the HS diet did not have significantly different interval from calving to conception ( $74 \pm 16.5$ days postpartum versus $59 \pm 7.5$ days postpartum) compared to cows fed the HS diet. 
Table 3.8: Effect of diet on probability of cows to be pregnant and to express abnormal milk progesterone profiles

\begin{tabular}{|c|c|c|c|c|c|}
\hline \multirow{2}{*}{$\begin{array}{c}\text { Treatment: } \\
\text { Parameters: }\end{array}$} & \multicolumn{4}{|c|}{ DIET } & \multirow[b]{2}{*}{$\boldsymbol{P}$} \\
\hline & $\mathbf{H S}^{\dagger}$ & $n_{1}^{\partial}$ & $\mathbf{H F}^{\dagger}$ & $n_{2}{ }^{\partial}$ & \\
\hline Probability for cows to be pregnant & $0.23(0.07,0.40)$ & 15 & $0.31(0.08,0.53)$ & 15 & 0.59 \\
\hline $\begin{array}{l}\text { Probability for cows to express } \\
\text { abnormal milk progesterone profile }\end{array}$ & $0.65(0.47,0.86)$ & 15 & $0.27(0.09,0.45)$ & 15 & 0.03 \\
\hline
\end{tabular}

${ }^{\dagger}$ Columns are means with $95 \%$ Confidence Interval (CI).

${ }^{\partial} n_{1}$ and $n_{2}$ are the numbers of animals fed with HS and HF diets, respectively.

Table 3.9: Effect of diet and BCS at calving (CONDITION) on days to first oestrus

\begin{tabular}{|c|c|c|c|c|c|c|c|c|c|c|}
\hline \multirow{2}{*}{$\begin{array}{l}\text { Treatments: } \\
\text { Parameters: }\end{array}$} & \multicolumn{5}{|c|}{ DIET } & \multicolumn{5}{|c|}{ CONDITION } \\
\hline & $\mathbf{H S}^{\dagger}$ & $n_{1}^{\partial}$ & $\mathbf{H F}^{\dagger}$ & $\boldsymbol{n}_{2}^{\partial}$ & $\boldsymbol{P}$ & $\mathbf{F A T}^{\dagger}$ & $n_{3}{ }^{\ddagger}$ & THIN $^{\dagger}$ & $n_{4} \neq$ & $\boldsymbol{P}$ \\
\hline $\begin{array}{l}\text { Days to first } \\
\text { oestrus } \\
(\text { days } \\
\text { postpartum })\end{array}$ & $58.2(50.2,66.2)$ & 13 & $51.2(44.5,57.9)$ & 15 & 0.19 & $53.3(46.7,59.9)$ & 13 & $56.1(47.6,64.7)$ & 15 & 0.26 \\
\hline
\end{tabular}


Table 3.10: Effect of BCS at calving (CONDITION) on circulating insulin, glucagon, GH, IGF-I, and leptin

\begin{tabular}{|c|c|c|c|c|c|c|}
\hline \multirow{2}{*}{$\begin{array}{c}\text { Treatment: } \\
\text { Parameters: }\end{array}$} & \multicolumn{4}{|c|}{ CONDITION } & \multirow[b]{2}{*}{ SED $^{\ddagger}$} & \multirow[b]{2}{*}{$P$} \\
\hline & $\mathbf{F A T}^{\dagger}$ & $n_{1}^{\partial}$ & THIN $^{\dagger}$ & $n_{2}^{\partial}$ & & \\
\hline $\begin{array}{l}\text { Insulin } \\
(n g / m l)\end{array}$ & 0.43 & 274 & 0.47 & 153 & 0.040 & 0.19 \\
\hline $\begin{array}{c}\text { Glucagon } \\
(\mathrm{pg} / \mathrm{ml})\end{array}$ & 93.4 & 151 & 95.3 & 83 & 5.97 & 0.75 \\
\hline$\underset{(n g / m l)}{\mathbf{G H}}$ & 4.40 & 141 & 4.27 & 81 & 0.670 & 0.84 \\
\hline $\begin{array}{c}\text { IGF-I } \\
(n g / m l)\end{array}$ & 104.9 & 142 & 147.5 & 82 & 9.75 & 0.03 \\
\hline $\begin{array}{l}\text { Leptin } \\
(n g / m l)\end{array}$ & 1.72 & 272 & 1.41 & 15 & 0.298 & 0.30 \\
\hline
\end{tabular}


Table 3.11: Effect of diet on circulating insulin, glucagon, GH, IGF-I, and leptin

\begin{tabular}{|c|c|c|c|c|c|c|}
\hline \multirow{2}{*}{$\begin{array}{c}\text { Treatment: } \\
\text { Parameters: }\end{array}$} & \multicolumn{4}{|c|}{ DIET } & \multirow[b]{2}{*}{ SED $^{\ddagger}$} & \multirow[b]{2}{*}{$P$} \\
\hline & $\mathbf{H S}^{\dagger}$ & $n_{1}^{\partial}$ & $\mathbf{H F}^{\dagger}$ & $n_{2}^{\partial}$ & & \\
\hline $\begin{array}{l}\text { Insulin } \\
(n g / m l)\end{array}$ & 0.49 & 211 & 0.41 & 216 & 0.034 & 0.02 \\
\hline $\begin{array}{c}\text { Glucagon } \\
(p g / m l)\end{array}$ & 99.5 & 116 & 89.6 & 118 & 5.28 & 0.06 \\
\hline$\underset{(n g / m l)}{\mathbf{G H}}$ & 4.54 & 106 & 4.15 & 116 & 0.650 & 0.54 \\
\hline $\begin{array}{c}\text { IGF-I } \\
(n g / m l)\end{array}$ & 132.0 & 105 & 122.7 & 119 & 13.16 & 0.48 \\
\hline $\begin{array}{l}\text { Leptin } \\
(n g / m l)\end{array}$ & 1.73 & 208 & 1.43 & 216 & 0.312 & 0.35 \\
\hline
\end{tabular}


Table 3.12: Effect of BCS at calving (CONDITION) on circulating glucose, urea, $\beta$-hydroxy butyrate (BOHB), and nonesterified fatty acids (NEFA).

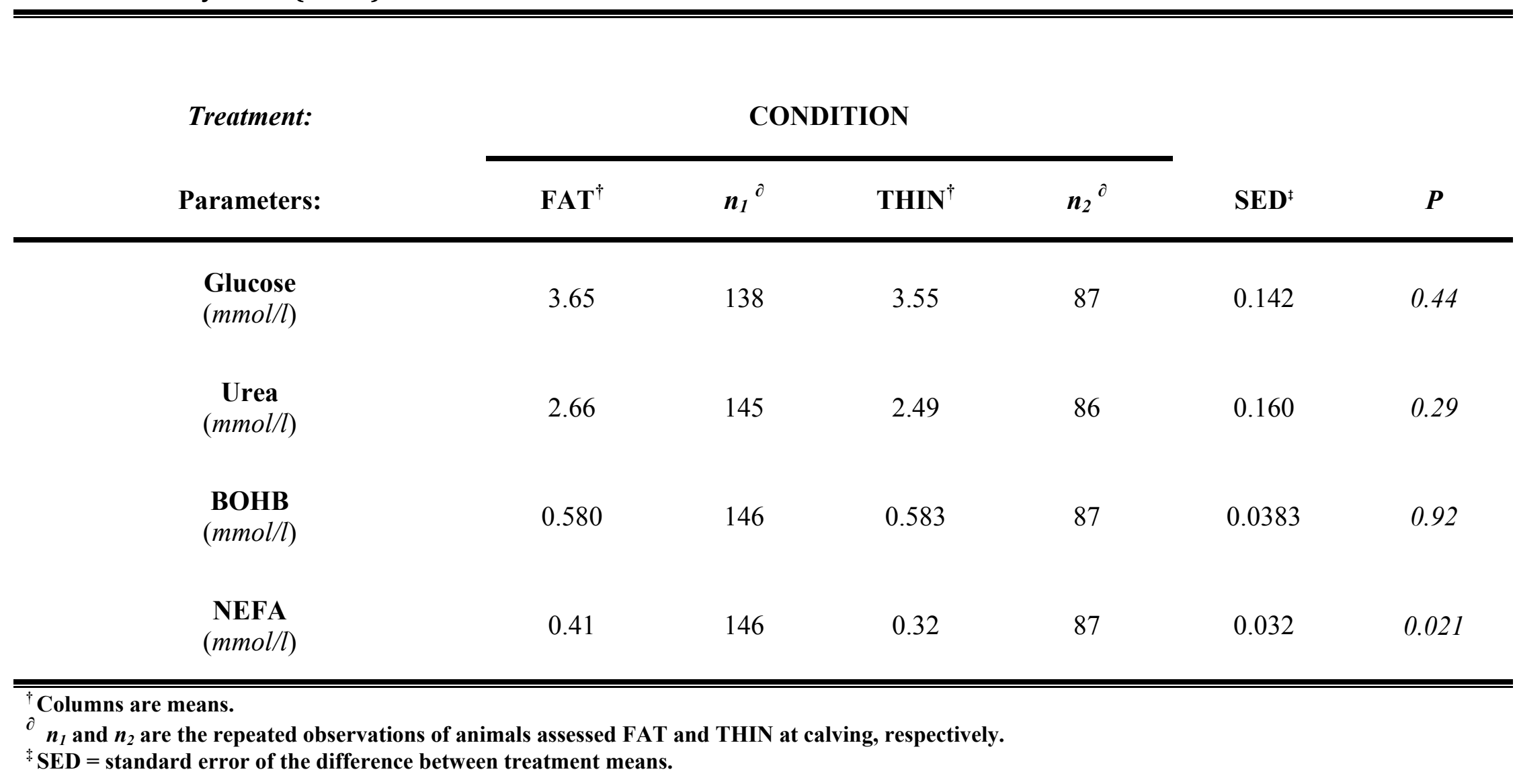


Table 3.13: Effect of diet on circulating glucose, urea, $\beta$-hydroxy butyrate (BOHB), and non-esterified fatty acids (NEFA).

\begin{tabular}{|c|c|c|c|c|c|c|}
\hline \multirow{2}{*}{$\begin{array}{c}\text { Treatment: } \\
\text { Parameters: }\end{array}$} & \multicolumn{4}{|c|}{ DIET } & \multirow[b]{2}{*}{ SED } & \multirow[b]{2}{*}{$\boldsymbol{P}$} \\
\hline & $\mathbf{H S}^{\dagger}$ & $n_{1}^{\partial}$ & $\mathbf{H F}^{\dagger}$ & $n_{2}^{\partial}$ & & \\
\hline $\begin{array}{l}\text { Glucose } \\
(\mathrm{mmol} / \mathrm{l})\end{array}$ & 3.60 & 115 & 3.61 & 110 & 0.114 & 0.92 \\
\hline $\begin{array}{c}\text { Urea } \\
(\mathrm{mmol} / \mathrm{l})\end{array}$ & 2.83 & 114 & 2.32 & 117 & 0.146 & 0.01 \\
\hline $\begin{array}{c}\text { ВОНB } \\
(\mathrm{mmol} / \mathrm{l})\end{array}$ & 0.485 & 115 & 0.650 & 118 & 0.0404 & 0.01 \\
\hline $\begin{array}{c}\text { NEFA } \\
(\mathrm{mmol} / \mathrm{l})\end{array}$ & 0.306 & 115 & 0.309 & 118 & 0.0262 & 0.90 \\
\hline
\end{tabular}

\footnotetext{
Columns are means.

$n_{1}$ and $n_{2}$ are the repeated observations of animals fed with HS and HF diets, respectively.

${ }^{\star}$ SED $=$ standard error of the difference between treatment means.
} 
Table 3.14: Effect of BCS at calving (CONDITION) on dry matter intake (DMI), live weight (LWT), milk yield, BCS, nadir BCS, nadir week, and postpartum body condition loss $(\triangle \mathrm{BCS})$

\begin{tabular}{|c|c|c|c|c|c|c|}
\hline \multirow{2}{*}{$\begin{array}{c}\text { Treatment: } \\
\text { Parameters: }\end{array}$} & \multicolumn{4}{|c|}{ CONDITION } & \multirow[b]{2}{*}{ SED } & \multirow[b]{2}{*}{$P$} \\
\hline & $\mathbf{F A T}^{\dagger}$ & $n_{1}^{\partial}$ & THIN $^{\dagger}$ & $n_{2}^{\partial}$ & & \\
\hline $\begin{array}{l}\text { DMI } \\
(\mathrm{kg} / \mathrm{d})\end{array}$ & 22.52 & 294 & 21.12 & 166 & 1.401 & 0.18 \\
\hline $\begin{array}{l}\text { Milk Yield } \\
\qquad(\mathrm{kg} / \mathrm{d})\end{array}$ & 47.7 & 294 & 40.5 & 166 & 2.06 & 0.02 \\
\hline $\begin{array}{c}\mathbf{L W T} \\
(\mathrm{kg})\end{array}$ & 658 & 294 & 664 & 166 & 7.1 & 0.41 \\
\hline $\begin{array}{c}\text { BCS } \\
\text { (units 1-5) }\end{array}$ & 2.66 & 304 & 2.36 & 169 & 0.069 & 0.003 \\
\hline $\begin{array}{c}\text { Nadir BCS } \\
\text { (units 1-5) }\end{array}$ & 2.30 & 15 & 2.02 & 15 & 0.076 & 0.017 \\
\hline $\begin{array}{l}\text { Nadir Week } \\
\quad(\text { weeks })\end{array}$ & 6.5 & 15 & 4.5 & 15 & 0.59 & 0.024 \\
\hline $\begin{array}{c}\Delta \mathbf{B C S} \\
\text { (units 1-5) }\end{array}$ & 0.82 & 15 & 0.47 & 15 & 0.103 & 0.025 \\
\hline
\end{tabular}

†Columns are means.

$n_{1}$ and $n_{2}$ are the observations of animals assessed FAT and THIN at calving, respectively.

${ }^{\ddagger}$ SED $=$ standard error of the difference between treatment means. 
Table 3.15: Effect of diet on dry matter intake (DMI), live weight (LWT), milk yield, BCS, nadir BCS, nadir week, and postpartum body condition loss $(\triangle B C S)$

\begin{tabular}{|c|c|c|c|c|c|c|}
\hline \multirow{2}{*}{$\begin{array}{l}\text { Treatment: } \\
\text { Parameters: }\end{array}$} & \multicolumn{4}{|c|}{ DIET } & \multirow[b]{2}{*}{ SED } & \multirow[b]{2}{*}{$P$} \\
\hline & $\mathbf{H S}^{\dagger}$ & $n_{1}^{\partial}$ & $\mathbf{H F}^{\dagger}$ & $n_{2}^{\partial}$ & & \\
\hline $\begin{array}{c}\text { DMI } \\
(\mathrm{kg} / \mathrm{d})\end{array}$ & 21.50 & 228 & 22.15 & 232 & 0.957 & 0.50 \\
\hline $\begin{array}{l}\text { Milk Yield } \\
\quad(\mathrm{kg} / \mathrm{d})\end{array}$ & 45.4 & 228 & 42.8 & 232 & 2.56 & 0.31 \\
\hline $\begin{array}{c}\mathbf{L W T} \\
(\mathrm{kg})\end{array}$ & 658 & 228 & 664 & 232 & 7.2 & 0.37 \\
\hline $\begin{array}{c}\text { BCS } \\
\text { (units 1-5) }\end{array}$ & 2.44 & 240 & 2.58 & 233 & 0.099 & 0.17 \\
\hline $\begin{array}{c}\text { Nadir BCS } \\
\text { (units 1-5) }\end{array}$ & 2.11 & 15 & 2.27 & 15 & 0.084 & 0.19 \\
\hline $\begin{array}{c}\text { Nadir Week } \\
\quad(\text { weeks })\end{array}$ & 4.9 & 15 & 6.6 & 15 & 0.74 & 0.12 \\
\hline $\begin{array}{c}\Delta \mathbf{B C S} \\
\text { (units 1-5) }\end{array}$ & 0.76 & 15 & 0.63 & 15 & 0.112 & 0.43 \\
\hline
\end{tabular}


Table 3.16: Effects of insulin, parity, BCS at calving, and diet on probability of cows to be pregnant

\begin{tabular}{|c|c|c|c|c|c|}
\hline \multirow[b]{2}{*}{ Parameters in the model: } & \multirow[b]{2}{*}{$\exp (b)^{\dagger}$} & \multicolumn{2}{|c|}{$95 \%$ CI for $\exp (b)$} & \multirow[b]{2}{*}{$\begin{array}{c}\text { Beta }(b) \\
\text { coefficient }\end{array}$} & \multirow[b]{2}{*}{$\boldsymbol{P}$} \\
\hline & & Lower & Upper & & \\
\hline Constant & & & & 8.13 & 0.01 \\
\hline Insulin & 0 & 0 & 0.03 & -16.01 & 0.01 \\
\hline Parity & 0.21 & 0.05 & 0.85 & -1.54 & 0.03 \\
\hline BCS at calving $^{\partial}$ & 52.32 & 5.79 & 472.38 & 3.96 & 0.001 \\
\hline Diet $^{\diamond}$ & 0.73 & 0.10 & 5.34 & -0.31 & 0.76 \\
\hline
\end{tabular}

Likelihood ratio test $\left(\chi^{2}=16.56, d f=4, P=0.002\right) ;$ Hosmer \& Lemeshow test for fitness $\left(\chi^{2}=5.97, d f=8, P=0.65\right) ; \quad$ AUC $C_{R o C}=0.85 \pm 0.08$

${ }^{\dagger} \exp (b)$ is the odds ratio

${ }^{\partial}$ BCS at calving is a variable with two levels; $0=$ FAT and $1=$ THIN

${ }^{\diamond}$ Diet is a variable with two levels; $0=\mathrm{HS}$ and $1=\mathrm{HF}$ 


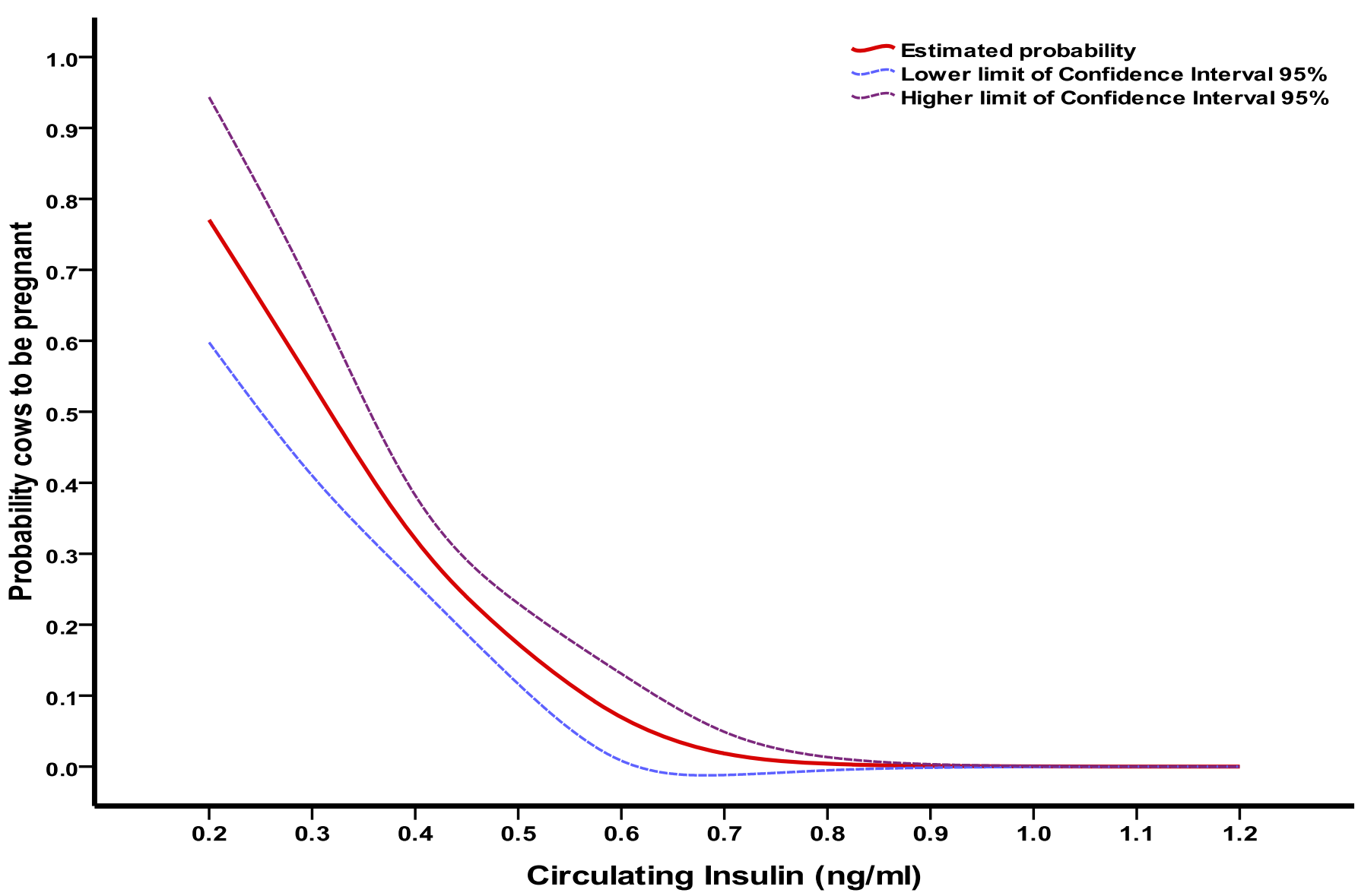

Figure 3.3: Effect of circulating insulin on probability of cows to be pregnant $\left(\mathrm{n}=30, P=0.01, \mathrm{AUC}_{R O C}=0.85 \pm 0.08, L R\left(\chi^{2}=16.56, d f=4\right.\right.$, $P=0.002)$, Hosmer \& Lemeshow test for fitness $\left(\chi^{2}=5.97, d f=8, P=0.65\right)$, multiple logistic regression model with Bernoulli error distribution, logit link function, and robust standard error estimation). In this model, dependent variable was pregnancy $(0=$ non pregnant, $1=$ pregnant $)$ whereas insulin and parity were added as continuous covariates, and BCS at calving and diet as factors. Red solid line represents the estimated probability whereas blue and purple dashed lines show the upper and lower $95 \%$ Confidence Interval (CI). Optimum insulin concentration that maximized the probability of cows to be pregnant ranged from 0.2 to $0.3 \mathrm{ng} / \mathrm{ml}$, whereas insulin greater than $0.6 \mathrm{ng} / \mathrm{ml}$ tended to zero the probability of cows to be pregnant. Model parameter estimates are presented in Table 3.16 . 


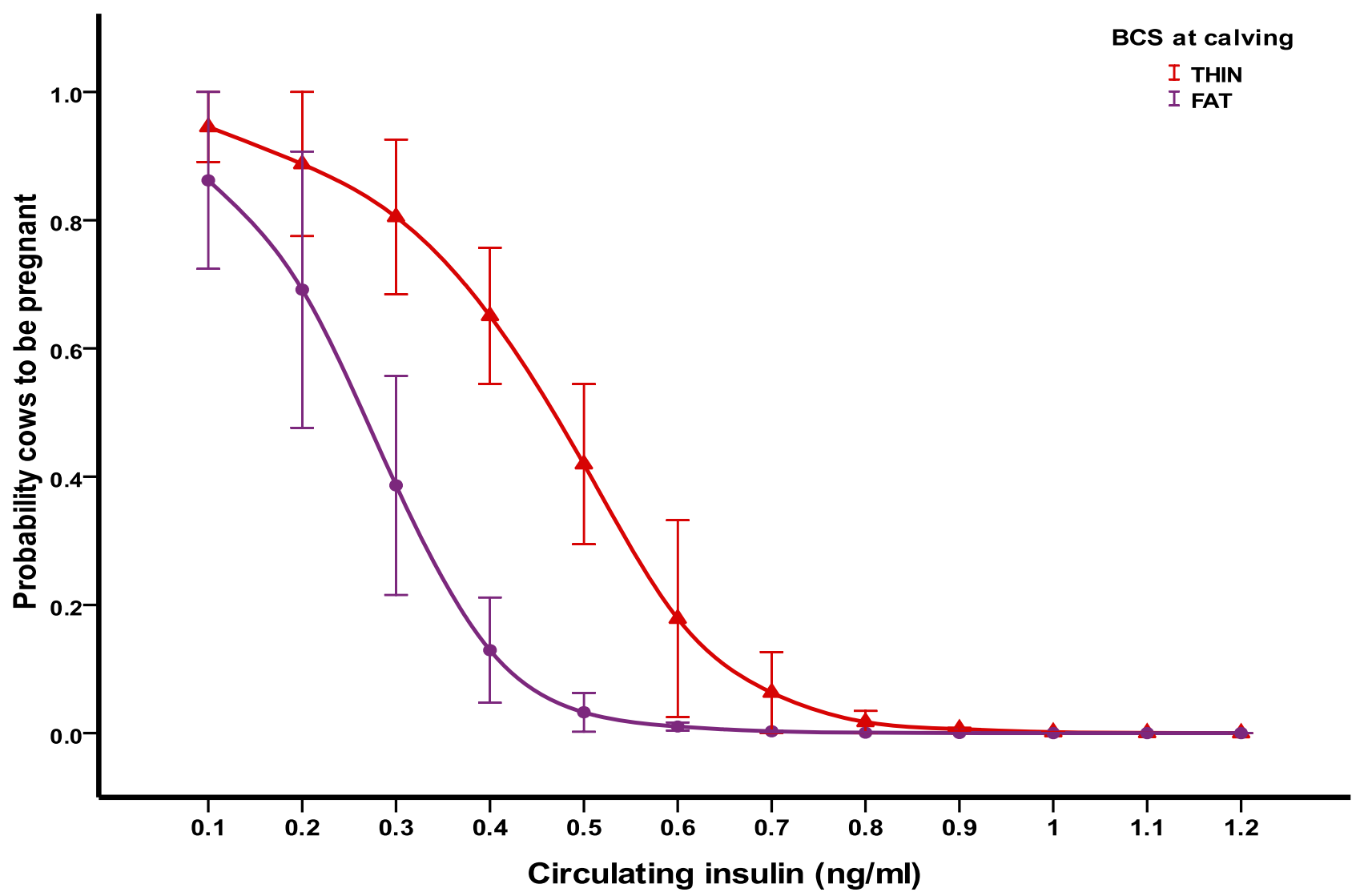

Figure 3.4: Probability of cows to be pregnant adjusted for different condition status at calving (FAT versus THIN) and circulating insulin concentrations $\left(\mathrm{n}=30, P=0.01, \mathrm{AUC}_{R O C}=0.85 \pm 0.08, L R\left(\chi^{2}=16.56, d f=4, P=0.002\right)\right.$, Hosmer \& Lemeshow test for fitness $\left(\chi^{2}=5.97\right.$, $d f=8, P=0.65)$, multiple logistic regression model with Bernoulli error distribution, logit link function, and robust standard error estimation). In this model, dependent variable was pregnancy $(0=$ non pregnant, $1=$ pregnant $)$ whereas insulin and parity were added as continuous covariates, and BCS at calving and diet as factors. Red triangles $(\boldsymbol{\Delta})$ represent marginal means \pm SE for THIN cows at calving, whereas purple dots $(\bullet)$ are marginal means \pm SE for FAT cows at calving. THIN cows at calving had higher probability to be pregnant than FAT cows at calving $(P<0.05)$, when the animals had approximately the same insulin concentration and insulin concentration ranged from 0.3 to $0.6 \mathrm{ng} / \mathrm{ml}$. Model parameter estimates are presented in Table 3.16. 


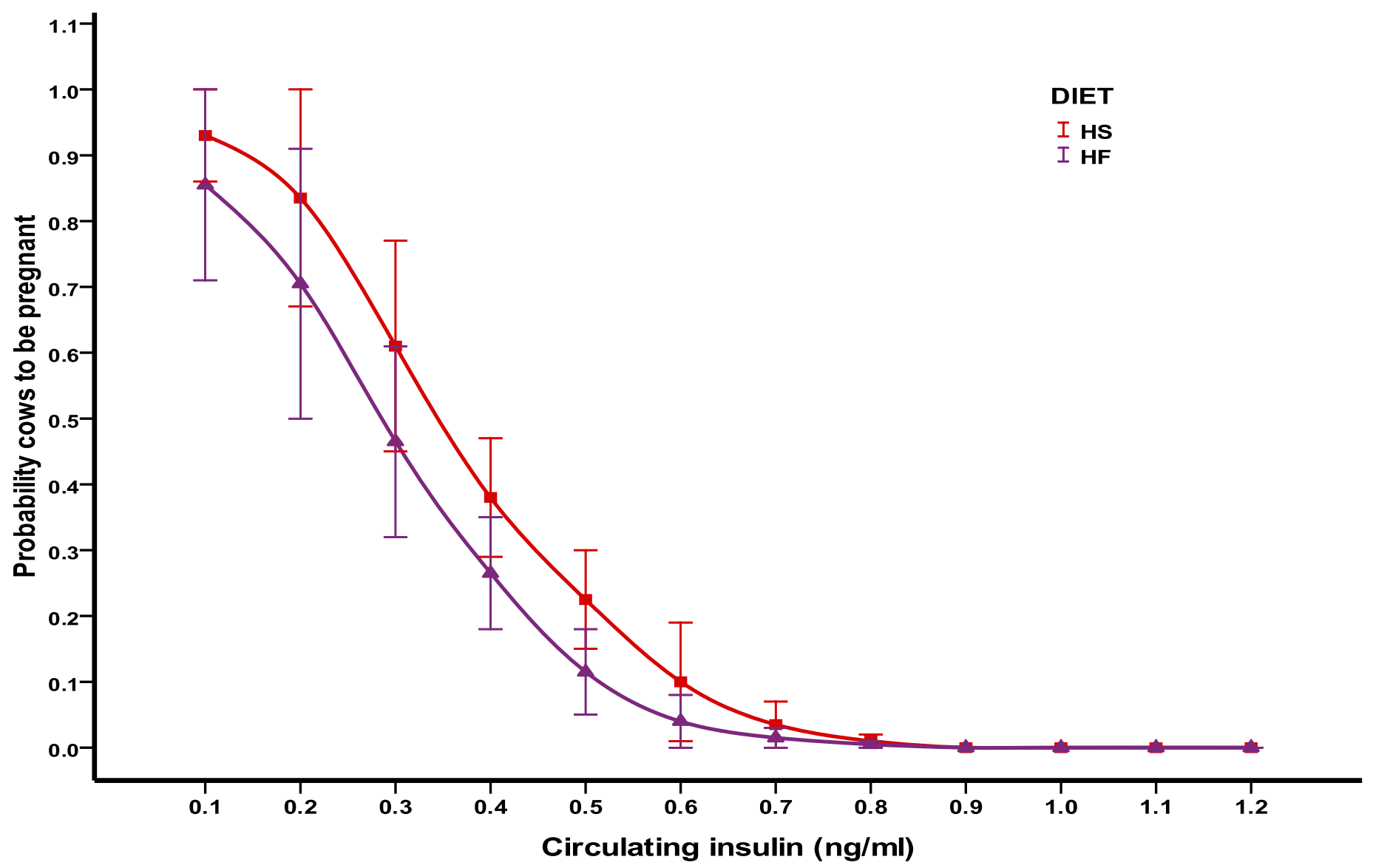

Figure 3.5: Probability of cows to be pregnant adjusted for different dietary treatments (HS versus HF) and circulating insulin concentrations $\left(\mathrm{n}=30, P=0.76, \mathrm{AUC}_{R O C}=0.85 \pm 0.08, L R\left(\chi^{2}=16.56, d f=4, P=0.002\right)\right.$, Hosmer \& Lemeshow test for fitness $\left(\chi^{2}=5.97, d f=8\right.$, $P=0.65)$, multiple logistic regression model with Bernoulli error distribution, logit link function, and robust standard error estimation). In this model, dependent variable was pregnancy $(0=$ non pregnant, $1=$ pregnant $)$ whereas insulin and parity were added as continuous covariates, and BCS at calving and diet as factors. Purple triangles $(\boldsymbol{\Delta})$ represent marginal means \pm SE for cows fed the HF diet, whereas red rectangles $(\square)$ are marginal means \pm SE for cows fed the HS diet. There is no effect of diet on probability of cows to be pregnant $(P>0.05)$, when the animals had approximately the same insulin concentration and insulin concentration ranged from 0.1 to $1.2 \mathrm{ng} / \mathrm{ml}$. Model parameter estimates are presented in Table 3.16. 


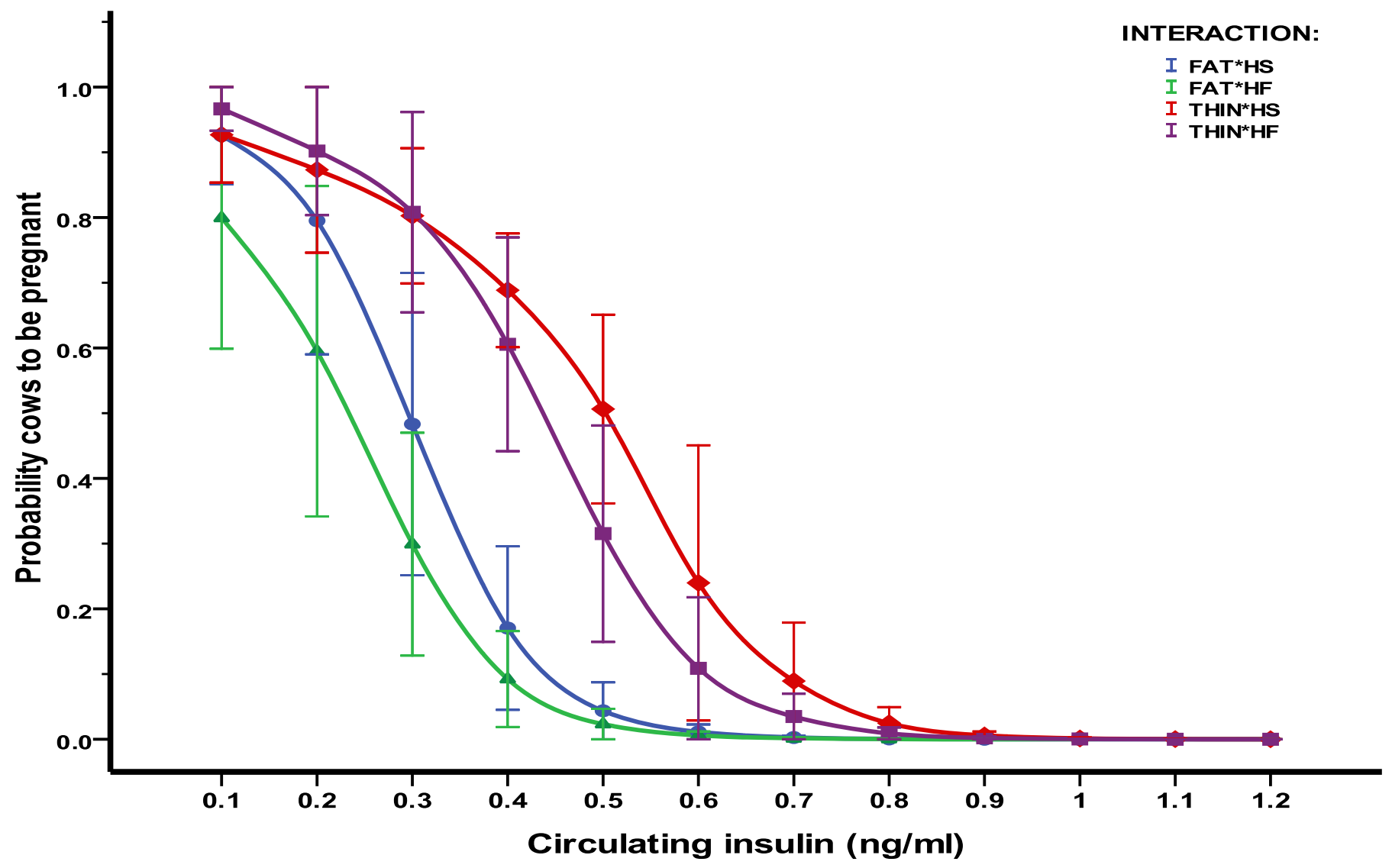

Figure 3.6: Probability of cows to be pregnant adjusted for different condition status at calving (FAT versus THIN), diets (HS versus HF), and circulating insulin concentrations $\left(\mathrm{n}=30, P=0.76, \mathrm{AUC}_{R O C}=0.85 \pm 0.08\right.$, LR $\left(\chi^{2}=16.56, d f=4, P=0.002\right)$, Hosmer \& Lemeshow test for fitness $\left(\chi^{2}=5.97, d f=8, P=0.65\right)$, multiple logistic regression model with Bernoulli error distribution, logit link function, and robust standard error estimation). In this model, dependent variable was pregnancy $(0=$ non pregnant, $1=$ pregnant $)$ whereas insulin and parity were added as continuous covariates, and BCS at calving, diet, and interaction (BCS at calving*diet) as factors. Red diamonds $(\diamond)$ are marginal means \pm SE for THIN cows fed the HS diet. Blue dots $(\bullet)$ are marginal means \pm SE for FAT cows fed the HS diet. Green triangles $(\Delta)$ represent marginal means \pm SE for FAT cows fed the HF diet. Purple rectangles $(\square)$ are marginal means \pm SE for THIN cows fed the HS diet. There was no effect of (BCS at calving*diet) on probability of cows to be pregnant $(P>0.05)$. 
Table 3.17: Effects of insulin, IGF-I, glucose, parity, BCS at calving, and diet on milk progesterone profile

\begin{tabular}{|c|c|c|c|c|c|}
\hline \multirow[b]{2}{*}{ Parameters in the model: } & \multirow[b]{2}{*}{$\exp (b)^{\dagger}$} & \multicolumn{2}{|c|}{$95 \%$ CI for $\exp (b)$} & \multirow[b]{2}{*}{$\begin{array}{c}\text { Beta }(b) \\
\text { coefficient }\end{array}$} & \multirow[b]{2}{*}{$\boldsymbol{P}$} \\
\hline & & Lower & Upper & & \\
\hline Constant & & & & 13.67 & 0.057 \\
\hline Insulin & 30,517 & 1.30 & $\sim+\infty\left(1.04 * 10^{9}\right)$ & 10.32 & 0.044 \\
\hline IGF-I & 0.97 & 0.937 & 1.004 & -0.03 & 0.087 \\
\hline Glucose & 0.007 & 0.0001 & 0.311 & -5.02 & 0.011 \\
\hline Parity & 8.59 & 1.49 & 49.44 & 2.15 & 0.016 \\
\hline BCS at calving ${ }^{\partial}$ & 0.014 & 0.0007 & 0.284 & -4.23 & 0.005 \\
\hline Diet $^{\diamond}$ & 0.079 & 0.004 & 1.778 & -2.53 & 0.110 \\
\hline
\end{tabular}

Likelihood ratio test $\left(\chi^{2}=13.79, d f=6, P=0.032\right) ;$ Hosmer \& Lemeshow test for fitness $\left(\chi^{2}=3, d f=8, P=0.934\right) ; A U C_{R o c}=0.91 \pm 0.05$

${ }^{\dagger} \exp (b)$ is the odds ratio

${ }^{\partial}$ BCS at calving is a variable with two levels; $0=$ FAT, $1=$ THIN

$\diamond$ Diet is a variable with two levels; $0=\mathrm{HS}, 1=\mathrm{HF}$ 


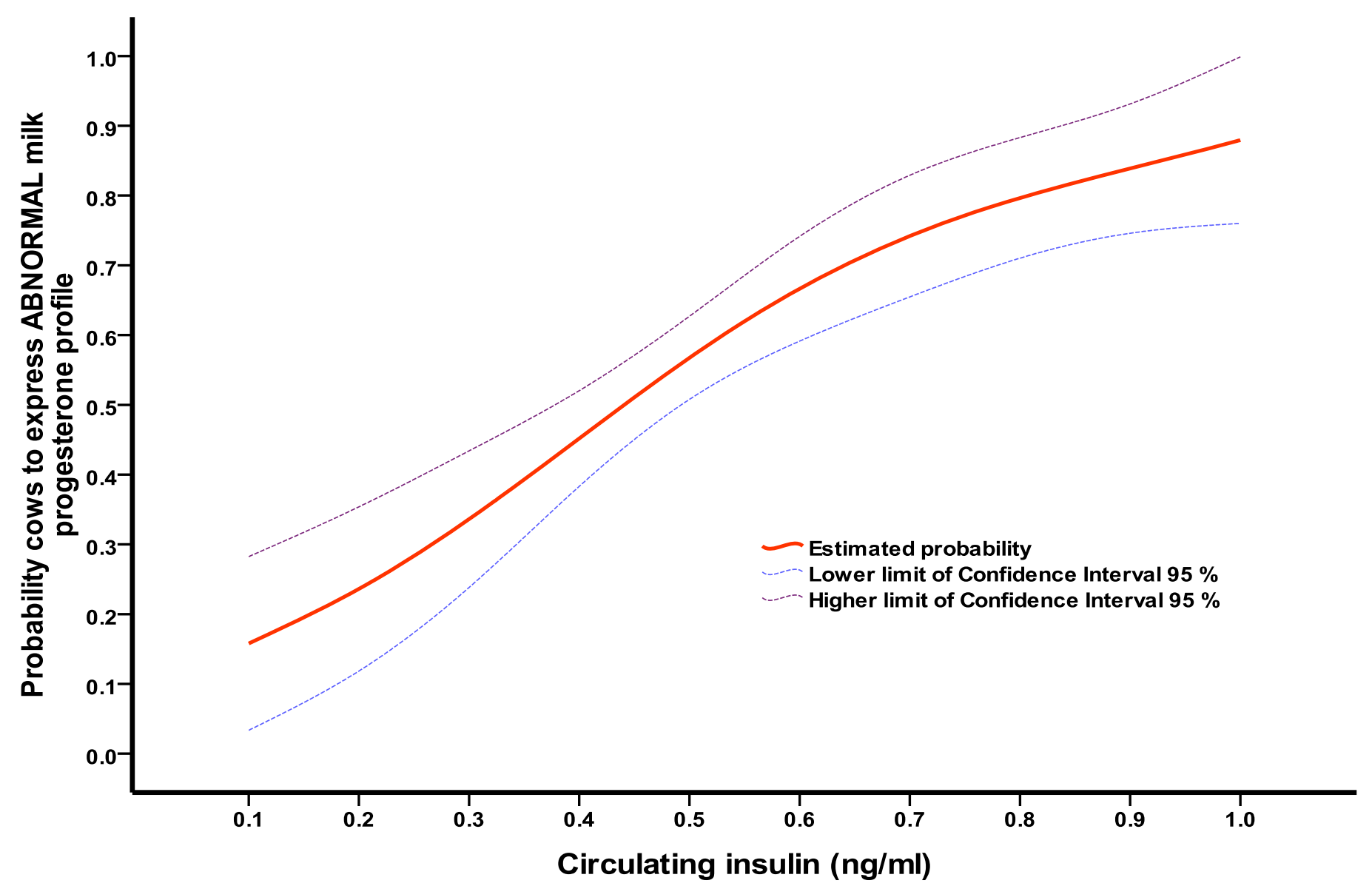

Figure 3.7: Effect of circulating insulin on probability for cows to express abnormal milk progesterone profile (n=30, $P=0.044$, $\mathrm{AUC}_{R O C}=0.91 \pm 0.05, L R\left(\chi^{2}=13.79, d f=6, P=0.032\right)$, Hosmer \& Lemeshow test for fitness $\left(\chi^{2}=3, d f=8, P=0.934\right)$, multiple logistic regression model with Bernoulli error distribution, logit link function, and robust standard error estimation). In this model, dependent variable was milk progesterone profile $(0=$ normal, $1=$ abnormal $)$ whereas insulin, IGF-I, glucose, and parity were added as covariates, and BCS at calving and diet as factors. Red solid line represents the estimated probability whereas blue and purple dashed lines show the upper and lower $95 \%$ Confidence Interval (CI). Optimum insulin concentration that minimized the probability for cows to express abnormal milk progesterone profile ranged from 0.1 to $0.3 \mathrm{ng} / \mathrm{ml}$, whereas insulin greater than 0.5 to $0.6 \mathrm{ng} / \mathrm{ml}$ tended to maximize the probability for cows to express abnormal milk progesterone profile. Model parameter estimates are presented in Table 3.17. 


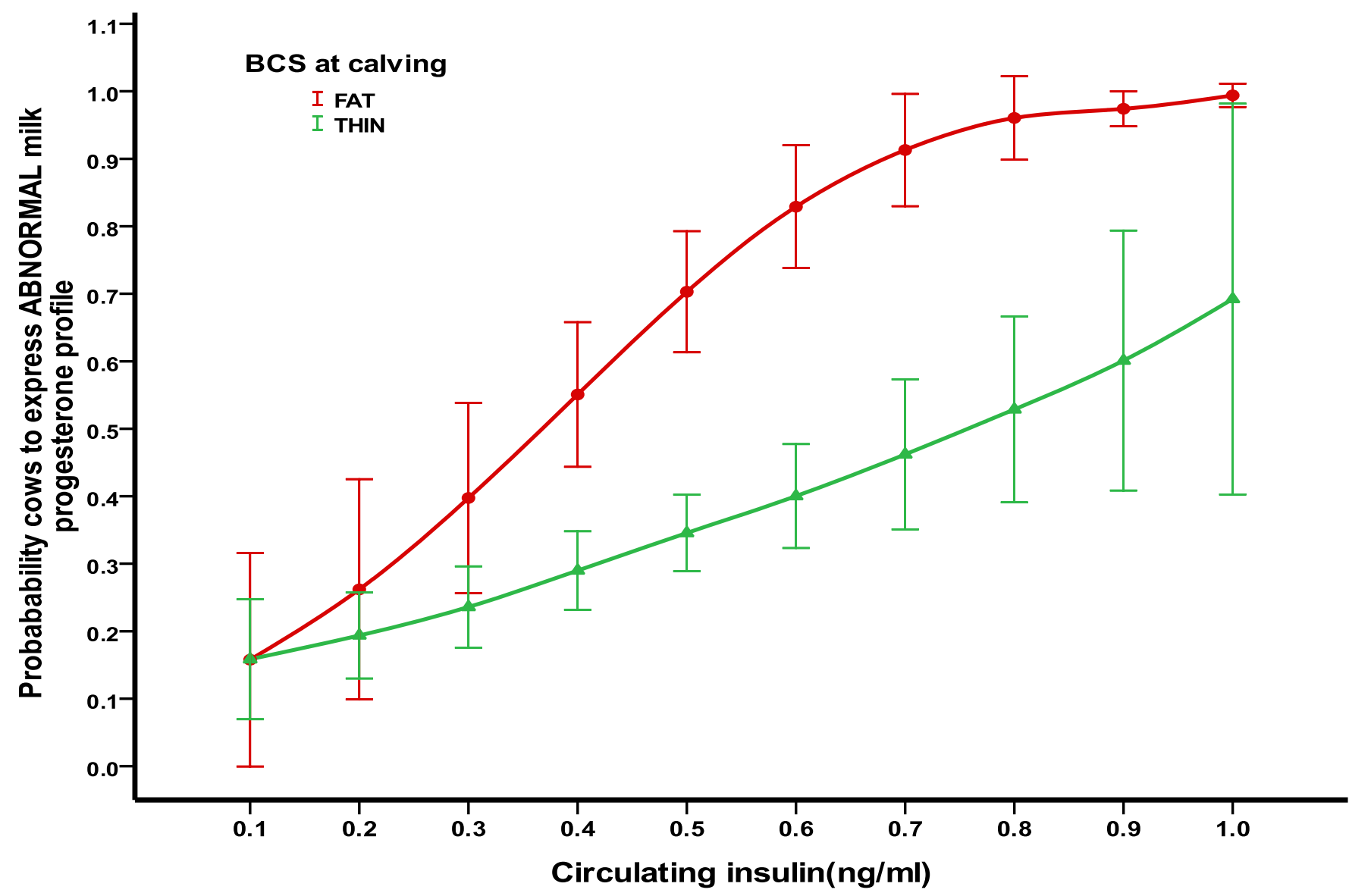

Figure 3.8: Probability for cows to express abnormal milk progesterone profile adjusted for different condition status at calving (FAT versus THIN) and circulating insulin concentrations $\left(\mathrm{n}=30, P=0.014, \mathrm{AUC}_{R O C}=0.91 \pm 0.05, L R \quad\left(\chi^{2}=13.79, d f=6, P=0.032\right)\right.$, Hosmer \& Lemeshow test for fitness $\left(\chi^{2}=3, d f=8, P=0.934\right)$, multiple logistic regression model with Bernoulli error distribution, logit link function, and robust standard error estimation). In this model, dependent variable was milk progesterone profile $(0=$ normal, $1=$ abnormal $)$ whereas insulin, IGF-I, glucose, and parity were added as covariates, and BCS at calving and diet as factors. Green triangles $(\boldsymbol{\Delta})$ represent marginal means \pm SE for THIN cows at calving, whereas red dots $(\bullet)$ are marginal means \pm SE for FAT cows at calving. THIN cows at calving had lower probability to express abnormal milk progesterone profile than FAT cows at calving $(P<0.05)$, when the animals had approximately the same insulin concentration and insulin concentration ranged from 0.4 to $0.9 \mathrm{ng} / \mathrm{ml}$. Model parameter estimates are presented in Table 3.17 . 


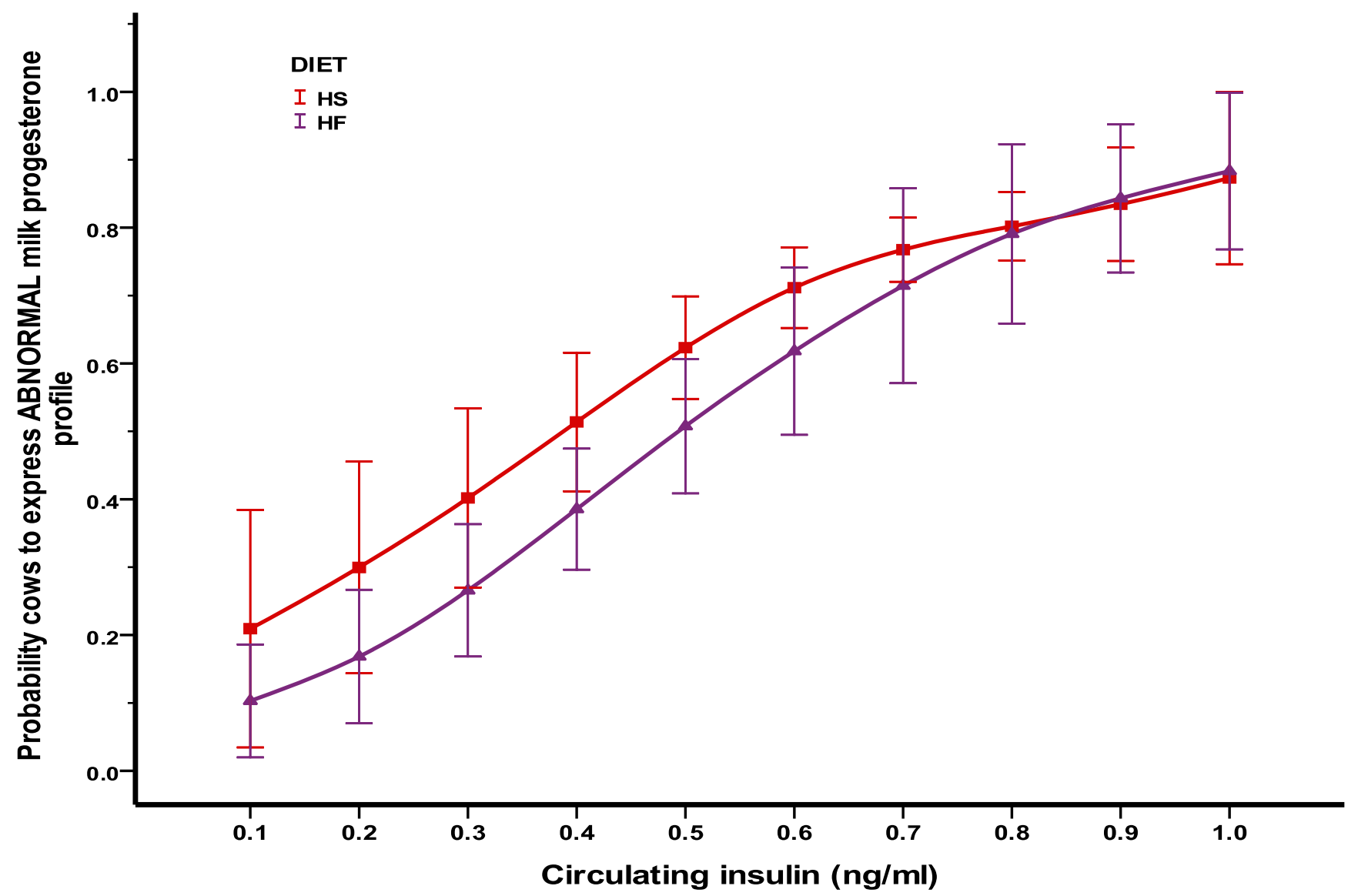

Figure 3.9: Probability for cows to express abnormal milk progesterone profile adjusted for different dietary treatments (HS versus $\mathrm{HF})$ and circulating insulin concentrations $\left(\mathrm{n}=30, \mathrm{AUC}_{R O C}=0.91 \pm 0.05, L R\left(\chi^{2}=13.79, d f=6, P=0.032\right)\right.$, Hosmer \& Lemeshow test for fitness $\left(\chi^{2}=3, d f=8, P=0.934\right)$, multiple logistic regression model with Bernoulli error distribution, logit link function, and robust standard error estimation). In this model, dependent variable was milk progesterone profile $(0=$ normal, $1=$ abnormal $)$ whereas insulin, IGF-I, glucose, and parity were added as covariates, and BCS at calving and diet as factors. Purple triangles ( $\mathbf{\Delta})$ represent marginal means \pm SE for cows fed the HF diet, whereas red rectangles ( $\square)$ are marginal means \pm SE for cows fed the HS diet. There is no effect of diet on probability for cows to express abnormal milk progesterone profile $(P>0.05)$, when the animals had approximately the same insulin concentration and insulin concentration ranged from 0.1 to $1.2 \mathrm{ng} / \mathrm{ml}$. Model parameter estimates are presented in Table 3.17. 


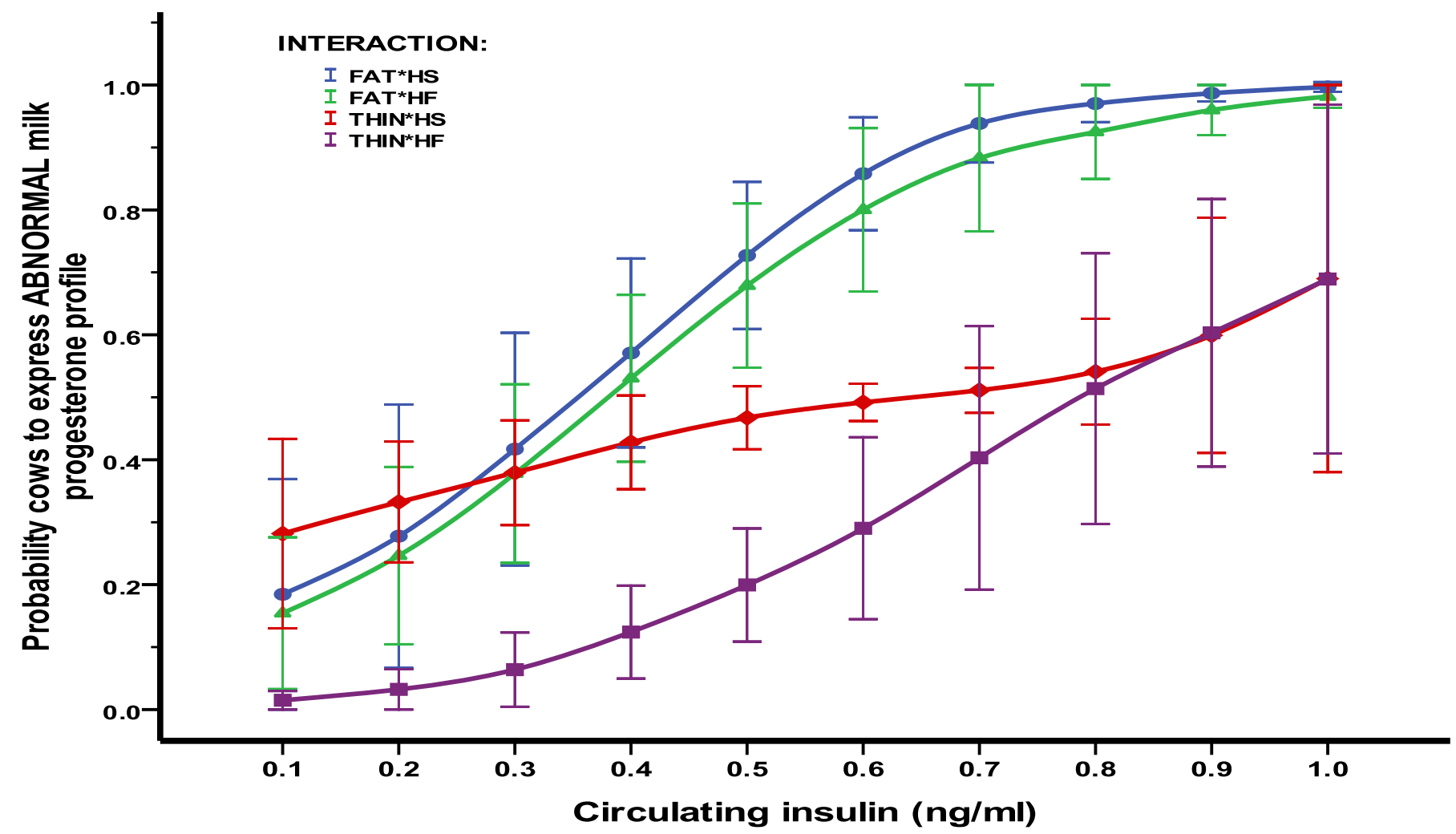

Figure 3.10: Probability for cows to express abnormal milk progesterone profile adjusted for different condition status at calving (FAT versus THIN), diets (HS versus HF), and circulating insulin concentrations $\left(\mathrm{n}=30, \mathrm{AUC}_{R O C}=0.91 \pm 0.05, L R\left(\chi^{2}=13.79, d f=6, P=0.032\right)\right.$, Hosmer \& Lemeshow test for fitness $\left(\chi^{2}=3, d f=8, P=0.934\right)$, multiple logistic regression model with Bernoulli error distribution, logit link function, and robust standard error estimation). In this model, dependent variable was milk progesterone profile $(0=$ normal, $1=\mathrm{abnormal})$ whereas insulin, IGF-I, glucose, and parity were added as covariates, and BCS at calving, diet, and interaction (BCS at calving*diet) as factors. Red diamonds $(\diamond)$ are marginal means \pm SE for THIN cows fed the HS diet. Blue dots $(\bullet)$ are marginal means \pm SE for FAT cows fed the HS diet. Green triangles $(\Delta)$ represent marginal means \pm SE for FAT cows fed the HF diet. Purple rectangles $(\square)$ are marginal means \pm SE for THIN cows fed the HS diet. THIN cows at calving that fed the HF diet had lower probability to express abnormal milk progesterone profile than THIN cows at calving that fed the HS diet $(P<0.05)$, when the animals had approximately the same insulin concentration and insulin concentration ranged from 0.1 to $0.6 \mathrm{ng} / \mathrm{ml}$. Moreover, THIN cows at calving that fed the HF diet had lower probability to express abnormal milk progesterone profile than THIN cows at calving that fed the HS diet $(P<0.05)$, when insulin concentration ranged from 0.1 to $0.3 \mathrm{ng} / \mathrm{ml}$. 


\subsection{DISCUSSION}

\subsubsection{Effect of BCS at calving on circulating metabolic hormones, metabolites, and reproductive traits in dairy cows}

The present study utilized data generated by the study of Garnsworthy et al. (2009). Because the animals retrospectively allocated to BCS at calving groups, analysis performed to assess the consistency of BCS at calving treatments. This analysis clearly demonstrated that the retrospective classification of cows as FAT and THIN was not biased by the original dietary treatments (Table 3.5).

The present study showed that there was no effect of BCS at calving on days to first oestrus. In agreement with this result, Pedron et al. (1993), Ruegg et al. (1992b), and Ruegg \& Milton (1995) did not find any influence of BCS at calving on days to first oestrus in dairy cows. Grainger et al. (1982) found that increasing BCS at calving resulted in fewer days to first oestrus in dairy cows. Also, Garnsworthy \& Topps (1982) demonstrated that dairy cows calving at a medium BCS $(2.5-3.0 ; 1-5$ units) had significantly fewer days to first oestrus than cows with high or low BCS at calving. These latter results are not in line with the present study.

The present study showed that FAT cows at calving had shorter interval from calving to conception than THIN cows at calving. Primiparous fat dairy cows (BCS at calving $\geq 3.0 ; 1$ 5 units) had a shorter interval from calving to conception than primiparous lean dairy cows (BCS at calving $<3.0 ; 1-5$ units) in the study of Meikle et al. (2004), which is in line with the current study. In contrast, other studies have failed to find any relationship between BCS at calving and calving interval (Garnsworthy \& Jones, 1988; Ruegg \& Milton, 1995; Pedron et al., 1993).

The present study found that FAT cows at calving had lower probability to be pregnant within 120 days postpartum than THIN cows at calving. According to Buckley et al. 
(2003) cows with precalving BCS $>3.0$ (1-5 units) had lower probability of pregnancy after 42 day of breeding, which is in agreement with the present study.

According to Lamming \& Darwash (1998), cows with abnormal patterns in milk progesterone profile showed a significant deterioration in postpartum reproductive performance. Also, dairy cows with abnormal oestrous cycles had lower pregnancy rate compared to cows with normal oestrous cycles in the study of Garnsworthy et al. (2009) and postpartum BCS loss of Holstein cows was risk factor for a prolonged luteal phase in the study of Ledoux et al. (2011). The present study found that cows with normal milk progesterone profile had higher probability to be pregnant than cows with abnormal milk progesterone profile, and FAT cows had higher probability to express abnormal milk progesterone profile than THIN cows. Moreover, FAT cows had lower IGF-I than THIN cows and IGF-I had a negative impact (tendency) on the odds for cows to express abnormal milk progesterone profile. This finding may explain why FAT cows had higher probability to express abnormal milk progesterone profile than THIN cows. Finally, these results taken together may explain why in the present study FAT cows were found to have lower probability to be pregnant within 120 days postpartum than THIN cows.

In the present study, components of postpartum reproductive performance (probability for cows to be pregnant or express abnormal milk progesterone profile, and interval from calving to conception) were influenced by BCS at calving. Only days to first oestrus was unaffected by BCS at calving. This suggests that BCS at calving is a determinant factor for postpartum reproductive performance of dairy cows. Looking for the underlying explanation for the influence of BCS at calving on reproduction, the present study examined the effect of BCS at calving on metabolic hormones and metabolites, and productive traits. Of the metabolic hormones measured, only IGF-I was affected by BCS at calving. In agreement with this result, Meikle et al. (2004) and Lake et al. (2006) found that circulating insulin was unaffected by BCS at calving. Meikle et al. (2004) reported that circulating leptin was statistically higher in fat cows (BCS at calving $\geq 3 ; 1-5$ units) than in thin cows (BCS at calving $<3 ; 1-5$ units), which is not in agreement with the present study. A likely reason for these discrepancies between studies is that cows in the 
present study were over-conditioned at calving compared with the cows in the studies of Lake et al. (2006) and Meikle et al. (2004). Another explanation could be variation in breeds, diets, and production systems. It is known that circulating insulin and glucagon are modulated mostly by dietary starch intake and its interaction with blood sugar (Brockman 1978, 1979; Marieb \& Hoehn, 2007; Aronoff et al. 2004), and that is possibly the reason for no effect of animal condition in this experiment. Circulating GH and leptin may be associated with BCS at parturition (Garnsworthy et al., 2008a), but this is only when differences of BCS at calving are great (Ryan et al., 1994). In the present study condition differences at calving were minimal, which could account for the lack of influence of condition at parturition on circulating GH and leptin. According to Hill (2004) the correlation between leptin and body condition appears to weaken postpartum when many other physiological influences are in action, and this is possibly an extra reason for no effect of BCS at parturition on circulating leptin.

FAT cows at calving had lower circulating IGF-I than THIN cows at calving throughout the experimental period, which could also explain why THIN cows showed superior reproductive performance compared with FAT cows. Lake et al. (2006) found that serum IGF-I was greater in fat cows (BCS at calving: 6.0; 1-10 units) than in thin cows (BCS at calving: 4.0; 1-10 units) and Meikle et al. (2004) noted that lean dairy cows at calving (BCS $<3 ; 1-5$ units) had lower circulating IGF-I than fat cows (BCS $\geq 3 ; 1-5$ units). These two results appear to contradict the finding of the present study. However, the majority of cows in the present study were over-conditioned at calving (BCS=3.38 \pm 0.2 ); and THIN cows at calving $(\mathrm{BCS}=3.00 \pm 0.08)$ were actually fat to moderate fat whereas FAT cows at calving $(\mathrm{BCS}=3.59 \pm 0.03)$ were over-fat. Therefore, the results of Lake et al. (2006) and Meikle et al. (2004) are, at least in part, in agreement with the result of the present study.

Of the metabolites examined in this experiment, only circulating NEFA was affected by BCS at calving. Busato et al. (2002) found that circulating NEFA was higher in fat cows (BCS one week ante partum $\geq 3.25 ; 1-5$ units) that lost greater than 0.75 units of BCS throughout the experimental period. Furthermore, the same study showed that circulating glucose and insulin were not influenced by BCS one week ante partum combined with 
different levels of BCS loss. In the study of Lake et al. (2006), circulating glucose and BOHB was not affected by BCS at calving, which is in agreement with the current study; however, they observed a trend for circulating NEFA to be higher in fat cows compared to thin cows, in contrast to the current results. Also, Meikle et al. (2004) in agreement with the current results did not find any effect of BCS at calving on circulating BOHB but there was a trend for circulating NEFA to be higher in fat cows. Pedron et al. (1993) noted that circulating glucose was unchanged among classes of cows with different BCS at calving, but circulating FFA tended to be higher only in over-conditioned cows at parturition (BCS=4; $1-5$ units).

The present study showed that there was no effect of BCS at calving on DMI and LWT, but milk yield, BCS, $\triangle \mathrm{BCS}$, nadir BCS, and nadir week were higher in FAT than THIN cows at calving. In line with these results, Meikle et al. (2004) found that lean cows at calving $(\mathrm{BCS}<3.0)$ had lower mean BCS during the experimental period, and fat cows at calving (BCS $\geq 3.0$ ) tended to lose more BCS. Also, Lake et al. (2005) demonstrated that thin beef cows at parturition maintained condition over the course of the study, whereas fat beef cows lost condition, but without any effect of BCS at parturition on milk yield. In agreement with the results of the present study Ruegg \& Milton (1995) showed that duration and magnitude of condition loss depended primarily on BCS at calving and was greater for cows that calved with higher BCS. Pedron et al. (1993) found that class of BCS at parturition (BCS at calving; 3.0, 3.5, and 4.0; 1-5 units) did not influence milk yield, but Waltner et al. (1993) showed exactly the opposite. According to Garnsworthy \& Jones (1987) quality of diet postcalving may influence the association between BCS at calving and milk production, and it is likely to be a reason for the variation in the results reported from different studies. Many studies demonstrated that higher postpartum BCS loss ( $\triangle \mathrm{BCS}$ ) accompanied higher milk production (Ruegg \& Milton, 1995; Domecq et al., 1997), in agreement with results of the current study.

In the present study, FAT cows at calving showed higher nadir BCS, longer nadir week, and smaller BCS change. Roche et al. $(2007 \mathrm{c}, 2009)$ proposed that the greater the calving and nadir BCS, and the smaller the BCS loss between calving and nadir, the shorter the 
postpartum anoestrous interval. According to Houghton et al. (1990) fatter beef cows at calving exhibited a shorter postpartum anoestrous interval. In line with these results FAT cows at calving in the present study had shorter interval from calving to conception than THIN cows at calving.

It is known that postpartum DMI decreases with increasing BCS at calving (Broster \& Broster, 1998; Garnsworthy, 2006). In the current study, however, DMI was unaffected by BCS at calving. It could be that the differences in BCS at calving between THIN and FAT cows were not large enough and the majority of the cows were over-conditioned at calving (mean BCS at calving $=3.38 \pm 0.20$ ). Thus, the difference in DMI between FAT and THIN cows at calving was smaller.

The results of the present study clearly indicate that THIN cows at calving ( $\mathrm{BCS} \leq 3.25)$ had better reproductive performance than FAT cows at calving (BCS $>3.25)$. However, BCS at calving had no effect on days to first oestrus and FAT cows at calving showed shorter interval from calving to conception than THIN cows at calving. Although, reproductive hormones were not measured by the present study, metabolic hormones, metabolites, and productive traits convincingly explain why THIN cows at calving expressed better reproductive performance than FAT cows at calving. Circulating NEFA was higher in FAT cows at calving. NEFA are used as an alternative energy source by the liver to preserve glucose (Hayirli, 2006), and the majority of circulating glucose is used preferentially by the mammary gland to form lactose (Bauman \& Currie, 1980). In the present study, FAT cows at calving lost more condition to support milk production and without increasing DMI. The depressed DMI in FAT cows at calving could be the direct effect of elevated circulating NEFA on hypothalamic neural centres controlling feeding behaviour (Ingvartsen \& Andersen, 2000; Krasnow \& Steiner, 2006). Also, elevated circulating NEFA may detrimentally affect follicular growth and development by acting directly on follicle cells (Leroy et al., 2008, 2010), which may explain the inferior reproductive performance of FAT cows at calving. Circulating IGF-I was higher in THIN cows at calving. The IGF-system plays an important role in follicle growth and development by acting directly on ovarian cells (Webb et al., 2004). Moreover, low circulating IGF-I 
negatively influences postpartum reproductive performance (Butler et al., 2000; Huszenicza et al., 2001; Meikle et al., 2004). To conclude, the present study demonstrated that THIN cows at calving (BCS $\leq 3.25$ ), which lost less than 0.5 units of BCS ( $\triangle \mathrm{BCS})$ during the first 4 months of lactation, had superior reproductive performance, and that was because of increased circulating IGF-I and decreased circulating NEFA. However, further work is required to determine the effects of BCS at calving on fertility.

\subsubsection{Effect of diet on circulating metabolic hormones, metabolites, and reproductive traits in dairy cows}

A secondary objective of the present study was to determine whether feeding a HS diet (designed to induce increasing circulating insulin) or a HF (designed to reduce circulating insulin) for the first 4 months postpartum could result in changing reproductive performance of dairy cows. The present study showed that cows fed HS diet had higher probability to express abnormal milk progesterone profile than cows fed HF diet, but there was no effect of diet on the probability of cows to be pregnant, days to first oestrus, and interval from calving to conception. Additionally, high circulating insulin was associated with higher odds for cows to express abnormal milk progesterone profile, and that may explain why cows fed the HS diet had higher probability to express abnormal milk progesterone profile than cows fed the HF diet. Moreover, cows fed the HS diet had insulin concentrations tended to be $0.5 \mathrm{ng} / \mathrm{ml}$, whereas cows fed the HF diet had insulin concentrations tended to be $0.4 \mathrm{ng} / \mathrm{ml}$ in the present study (Table 3.11). This difference in insulin concentrations between animals fed the HS and HF diet may explain why cows fed the HS diet had higher probability to express abnormal milk progesterone profile than cows fed the HF diet (Table 3.8 and Figure 3.7).

Cows fed the HS diet had higher circulating insulin than cows fed the HF diet, but there was no effect of diet on circulating GH, leptin, and IGF-I. This finding is in agreement with the results of Gong et al. (2002b) and Garnsworthy et al. (2008b). According to Garnsworthy et al. (2008a) and Lucy (2000), circulating GH and IGF-I are associated 
more to productive traits (such as milk yield, live weight and energy balance) than to diet composition, whereas leptin is mainly linked with BCS (Hill, 2004), and it is conflicting if it can be affected by diet (Chilliard et al., 2005). Also, glucose and insulin seem to play a key role in regulating leptin expression in ruminants (Chilliard et al., 2005). Milk yield, BCS, $\triangle \mathrm{BCS}$, LWT, and circulating glucose were not affected by diet in this experiment and maybe that is why circulating GH, leptin, and IGF-I were not influenced by diet. Failure of diet to influence circulating metabolic hormones (except insulin), may explain why reproductive performance was the same for the two dietary treatments, as it is known that circulating $\mathrm{GH}$, leptin, and IGF-I directly affect reproductive performance (Garnsworthy et al., 2008a).

The study of Gong et al. (2002b) used similar diets to the current study, but for a period of 50 days postpartum. Their study clearly demonstrated a beneficial effect of cows fed HS diet on early reproductive indices (i.e. increased proportion of cows ovulating within 50 days postpartum, decreased intervals from calving to first ovulation, and decreased intervals from calving to first service and to conception). However, in the study of Gong et al. (2002b) the subsequent fertility parameters (i.e. conception rate to first service and number of services required per conception) were not affected by diet and that finding is at least in part in agreement with the current study. Moreover, this finding may suggest that the beneficial effect of HS diet on cow fertility is constrained to the first 50 days postpartum. In general, the results of the present study concerning early reproductive indices are not in line with those of the study of Gong et al. (2002b). Cows fed the HS diet did not show shorter days to first oestrus or intervals from calving to conception and they had higher probability to express abnormal milk progesterone profile than cows fed HF diet. This latter result combined with the finding that cows with normal milk progesterone profile had higher probability to be pregnant than cows with abnormal milk progesterone profile may indicate that cows fed the HS diet are likely to have lower probability to be pregnant than cows fed the HF diet. However, this latter conclusion was not confirmed by the present study as diet had no effect on the probability cows to be pregnant. An explanation for the discrepancies between the two studies could be that in the present study 
the animals were dietary manipulated for longer (up to 2 times) than the animals of the study of Gong et al. (2002b).

In the study of Gong et al. (2002b) circulating insulin was $0.32 \pm 0.015 \mathrm{ng} / \mathrm{ml}$ and $0.21 \pm$ $0.011 \mathrm{ng} / \mathrm{ml}$ for the high yielding cows fed HS and HF diets, respectively. In the present study, mean insulin was $0.44 \pm 0.02 \mathrm{ng} / \mathrm{ml}$ and animals fed the HS diet had higher mean circulating insulin $(0.49 \pm 0.03 \mathrm{ng} / \mathrm{ml})$ than cows fed the HF diet $0.41 \pm 0.03 \mathrm{ng} / \mathrm{ml}$. According to Kaneko et al. (2008b), reference range of circulating insulin for cows varies from zero (undetectable) to $0.23 \mathrm{ng} / \mathrm{ml}$. Moreover, basal circulating insulin varied from $0.14 \pm 0.02 \mathrm{ng} / \mathrm{ml}$ to $0.15 \pm 0.03 \mathrm{ng} / \mathrm{ml}$, independently from diet and genetic merit for milk yield in the study of Chagas et al. (2009). That means circulating insulin was approximately two times higher than the reference range in the current study (or about three times higher than the mean insulin in the study of Chagas et al. (2009)), independently from the dietary treatments. Thus, the animals in the present study exceeded an optimum insulin concentration which was not surpassed by the animals in the study of Gong et al. (2002b), and this is another explanation for the discrepancies between the two studies.

Butler (2000) reported that increased circulating urea may lead to impaired fertility of cows as high plasma urea concentrations interfere with the normal inductive actions of progesterone on the microenvironment of the uterus, and therefore cause suboptimal conditions for supporting embryo development. Sinclair et al. (2000) suggested that exposure of oocytes in antral follicles to high levels of ammonia, prevents cleavage and blastocyst formation. In the current study, cows fed the HS diet had higher circulating urea than cows fed the HF diet and this may explain the higher probability to express abnormal milk progesterone profile observed in cows fed the HS diet.

BOHB was influenced by diet in the present study, and cows fed the HS diet had lower circulating BOHB than cows fed the HF diet. Increased BOHB is associated with NEB (Bell, 1995; Grummer, 1995), but there was no effect of diet on other metabolites or productive traits (milk yield, BCS, $\triangle \mathrm{BCS}$, and nadir BCS) to indicate higher body fat 
mobilization of cows fed the HF diet in this experiment. The reason why circulating BOHB was elevated in this study is not known. BOHB has no direct effect in reproductive performance (Leroy et al., 2008), and its higher concentration in cows fed HF diet cannot explain why these cows had lower probability to express ABNORMAL milk progesterone profile.

To conclude, the present study demonstrated that feeding dairy cows with a HS diet or a HF diet for the first 4 months of lactation would not result in changing reproductive performance (probability of cows to be pregnant, interval from calving to conception, and days to first oestrus were not affected by dietary treatment). This was mainly because important metabolic hormones (i.e. leptin, IGF-I, and GH), productive traits (milk yield, BCS, $\triangle \mathrm{BCS}$, nadir week, and nadir BCS), and metabolites (i.e. NEFA and glucose) were not influenced by diet. In addition, long-term moderately elevated insulin concentration induced by feeding dairy cows the HS diet might account for the higher probability to express abnormal milk progesterone profile. However, further work is required to determine the effects of HS and HF diets on fertility, and especially the association of chronic moderately elevated insulin concentration with reproductive performance.

\subsubsection{Reproductive performance and optimum insulin concentration}

The present study showed that pregnancy rate was negatively affected by insulin (Figure 3.3) but this effect was dependent on BCS at calving (Figure 3.4). Also, cows with high insulin had higher probabilities to express abnormal milk progesterone profile (Figure 3.7) and this effect was dependent on BCS at calving (Figure 3.8). These two results taken together may imply that there was an optimum insulin concentration necessary for normal reproductive performance in lactating dairy cows which was dependent on BCS at calving. According to Garnsworthy et al. (2009) there is a minimum insulin concentration (from 0.21 to $0.32 \mathrm{ng} / \mathrm{ml}$ ) that is necessary for commencement of normal ovarian activity in early lactating cows. In accordance with this observation, the present study presented that; (1) optimum insulin concentration that maximized the probability of cows to be pregnant 
varied from 0.2 to $0.3 \mathrm{ng} / \mathrm{ml}$, whereas insulin greater than $0.6 \mathrm{ng} / \mathrm{ml}$ tended to zero the probability of cows to be pregnant (Figure 3.3); and (2) optimum insulin concentration that minimized the probability for cows to express abnormal milk progesterone profile varied from 0.1 to $0.3 \mathrm{ng} / \mathrm{ml}$, whereas insulin greater than 0.4 to $0.6 \mathrm{ng} / \mathrm{ml}$ tended to maximize the probability for cows to express abnormal milk progesterone profile (Figure 3.7). Thus, insulin concentration that optimized fertility in lactating cows varied from 0.2 to $0.3 \mathrm{ng} / \mathrm{ml}$ while insulin concentration greater than $0.6 \mathrm{ng} / \mathrm{ml}$ impaired reproductive performance in lactating dairy cows. Moreover, the current study found that BCS at calving was a critical modulator of fertility in lactating dairy cows (Figure 3.4 and Figure 3.8), whereas reproductive performance (pregnancy rate and milk progesterone profile) of dairy cows was not affected by dietary treatments (Figure 3.5 and Figure 3.9) when insulin concentrations were about the same. Therefore, achieving a BCS of less than 3.25 units at calving is essential to ensure optimum cow fertility.

In the present study, mean insulin was $0.44 \pm 0.02 \mathrm{ng} / \mathrm{ml}$ whereas the actual percentage of pregnant cows was $26.7 \%$ and the actual percentage of cows expressed irregular oestrous cycles was $46.7 \%$. According to Figure 3.3 and Figure 3.7, the model estimated percentage of pregnant cows and the model estimated percentage of cows expressed irregular oestrous cycles was $(25.5 \pm 5) \%$ and $(49.9 \pm 6) \%$, respectively. Thus, the low percentage of pregnant cows and the high percentage of irregular oestrous cycles in the animals of the present study were due to insulin concentration exceeded the optimum $(0.2-0.3 \mathrm{ng} / \mathrm{ml})$ in terms of reproduction.

Another finding of the present study was that THIN cows at calving that fed the HF diet had lower probability to express abnormal milk progesterone profile than THIN cows at calving that fed the HS diet, when insulin concentration ranged from 0.1 to $0.6 \mathrm{ng} / \mathrm{ml}$ (Figure 3.10). Therefore, HF diet must be the preferable feeding strategy to maximaze reproductive performance of dairy cows when insulin concentration ranges from 0.1 to 0.6 $n g / m l$ and BCS at calving is equal or lower than 3.25 units. Moreover, because THIN cows at calving had lower probability to express abnormal milk progesterone profile than FAT cows at calving when circulating insulin was relatively high (0.4 to $0.9 \mathrm{ng} / \mathrm{ml}$ ) (Figure 3.8), 
body condition management must be aimed at a BCS of less than 3.25 units at calving. Alternatively, if the animals are FAT at calving, the best feeding strategy to ensure optimum fertility is the animals to be fed a diet that induces an optimum circulating insulin concentration $(0.2-0.3 \mathrm{ng} / \mathrm{ml})$. However, this approach is not easy to be achieved in the long term, and consequently obtaining a BCS of less than 3.25 units at calving must be considered the most realistic strategy. Thus, BCS at calving management has greater impact on cow fertility than postpartum dietary treatments such as HS and HF diets. This latter may imply that prepartum feeding strategy and BCS management are essential for optimal reproduction performance in lactating dairy cows.

\subsubsection{Conclusions}

In conclusion, results of this study support the concept that BCS at calving is a critical modulator of fertility in lactating dairy cows. THIN cows at calving $(\mathrm{BCS} \leq 3.25)$, which lost less than 0.5 units of BCS ( $\triangle \mathrm{BCS}$ ) during the first 4 months of lactation, had superior reproductive performance, and that was because of increased circulating IGF-I and decreased circulating NEFA. Moreover, this study clearly shows that there is an optimum insulin concentration $(0.2-0.3 \mathrm{ng} / \mathrm{ml})$ necessary for normal reproductive performance, while insulin concentration greater than $0.6 \mathrm{ng} / \mathrm{ml}$ impairs reproductive performance in lactating dairy cows. The results strongly suggest that optimal reproductive performance of dairy cow is dependent on insulin concentration and BCS at calving. Cow fertility was not affected by dietary treatments, mainly because optimum insulin concentration was exceeded by the animals of the present study. However, HF diet must be the preferable feeding strategy to maximize reproductive performance of dairy cows when insulin concentration ranges from 0.1 to $0.3 \mathrm{ng} / \mathrm{ml}$ and BCS at calving is equal or lower than 3.25 units. Finally, moderately elevated insulin concentration that exceeded optimum insulin concentration may explain the low percentage of pregnant cows and the high percentage of cows expressed abnormal oestrous cycles in this experiment. However, these results need to be investigated further. 


\section{Measurement of plasma and milk adiponectin, and the impact of lactation stage, diet, and body condition at calving on plasma adiponectin concentrations in dairy cows}

\subsection{INTRODUCTION}

Adiponectin, also known as Acrp30, apM1, and AdipoQ, is an adipokine mainly secreted by adipocytes (Berg et al., 2002; Kadowaki \& Yamauchi, 2005; Shetty et al., 2009). Adipokines are highly bioactive molecules and may be implicated in regulation of energy expenditure and homeostasis, immunity, and reproduction (Yokota et al., 2000; Mitchell et al., 2005; Antuna-Puente et al., 2008; Guzic et al., 2006; Kamada et al., 2008; Dridi \& Taouis, 2009). Although leptin and adiponectin are the main adipokines expressed in adipose tissue (Guzic et al., 2006; Ahima, 2006), adiponectin concentration in plasma is two to three times higher than other hormones (Ahima, 2006). Circulating adiponectin varies in different species (human; 3-30 $\mu \mathrm{g} / \mathrm{ml}$, rat; 10-30 $\mu \mathrm{g} / \mathrm{ml}$, horses; 1.3-4.8 $\mu \mathrm{g} / \mathrm{ml}$, dog; 20-40 $\mu \mathrm{g} / \mathrm{ml}$, cats; $1-4 \mathrm{ng} / \mathrm{ml}$ ) (Kamada et al., 2008; Mousavinasab et al., 2005; Combs et al., 2004; Goldstein \& Scalia, 2007; Ishioka et al., 2006; Kearns et al., 2006; Radin et al., 2009; Hoenig et al., 2007 ).

It is known that periparturient cows enter a period of negative energy balance accompanied by decreased insulin sensitivity (Bell, 1995; Veerkamp et al., 2003; Hayirli, 2006), and adiponectin is implicated in murine and human insulin resistance (Kadowaki \& Yamauchi, 2005). Lemor et al. (2009) reported that AdipoR1 and AdipoR2 mRNA abundance in adipose tissue decreased as high yielding cows moved from pregnancy to lactation. Raddatz et al., (2008) reported that plasma adiponectin concentration varied from $8.3 \pm 1.4$ $\mathrm{ng} / \mathrm{ml}$ to $16.0 \pm 2.7 \mathrm{ng} / \mathrm{ml}$ in early lactating cows and circulating adiponectin was 
significantly increased from week 1 to week 4 post partum, and declined to remain at 12$13 \mathrm{ng} / \mathrm{ml}$ until week 11 postpartum. Komatsu et al. (2007) showed that adiponectin mRNA expression in adipose tissue was higher in non-lactating cows than peak-lactation cows, but it was similar in early-lactation and late-lactation cows. The pattern of change of circulating adiponectin throughout the lactation cycle of dairy cows has not yet been determined.

Contrary to what happens for most adipokines, circulating values of adiponectin are higher in lean than in obese individuals (Berg et al., 2002; Matsuzawa et al., 2004; Kadowaki \& Yamauchi, 2005; Kadowaki et al., 2006; Shetty et al., 2009; Goldstain \& Scalia, 2007). Kearns et al. (2006) reported that adiponectin is inversely proportional to adiposity in horses. According to Ishioka et al. (2006) obese dogs showed low circulating adiponectin. Raddatz et al. (2008) proposed that circulating adiponectin is not correlated with BCS in early lactating cows. Circulating adiponectin was negatively correlated with body weight, body mass index, and insulin in humans (Berg et al , 2002; Matsuzawa et al., 2004; Guzic et al., 2006; Ahima, 2006), but the association of circulating adiponectin with body weight, BCS at calving, and metabolic profile in cattle or other ruminants have not yet been investigated.

Dietary factors may modulate circulating adiponectin (Reis et al., 2010). High consumption of magnesium (Qi et al., 2005; Cassidy et al., 2009), caffeine (Williams et al., 2008), n-3 PUFA (Duda et al., 2007), and dietary salt (Lely et al., 2007) are associated in humans with higher circulating adiponectin. According to Barnea et al. (2006) mice fed a High Fat diet exhibited elevated insulin resistance, although circulating adiponectin remained unchanged compared to controls. Jones et al. (2009) demonstrated that a high-fat diet increased maternal adiposity and circulating maternal leptin, but murine serum adiponectin was decreased from a period around the mating and throughout gestation. Pischon et al. (2005) showed that circulating adiponectin was negatively related to glycaemic load in men. It is known that ruminants regulate glucose homeostasis and lipid metabolism in different ways than other monogastric animals (Allen et al., 2005; Nafikov $\&$ Beitz, 2007). For example, many ruminant tissues preferentially utilize acetate rather 
than glucose, and VFA are the main products of organic matter fermentation (Lewis \& Hill 1983; Nafikov \& Beitz, 2007). The effect of dietary components on circulating adiponectin has not yet been demonstrated for any ruminant.

Adiponectin is assessed in human milk (Weyermann et al., 2006; Martin et al., 2006; Bronsky et al., 2006), and bovine mammary epithelial cells are expressed AdipoR1 (Ohtani et al., 2011). However, the presence of adiponectin in bovine milk has not yet been reported.

In this project, circulating and milk adiponectin were measured by using a commercially available human adiponectin kit (HADP-61 HK, Linco Research, Millipore, St. Charles, MO, USA). The presence of adiponectin in bovine milk was confirmed for first time. The main objective of the study was to test the hypothesis that as physiological state of cow dramatically changes by moving from pregnancy to calving and lactation, major physiological changes occur, which may be partially reflected in circulating adiponectin. Additionally, the influences of BCS at calving and diet on circulating adiponectin, and the relationships of circulating adiponectin with other metabolic hormones, metabolites, and productive traits were investigated. Kubota et al., (2007) showed that adiponectin stimulate food intake in mouse. A second hypothesis was that circulating adiponectin would be associated with DMI in lactating dairy cows. Evidence may indicate that the relationship between GH and adiponectin is negative in human and rat (Lam et al., 2004; Nilsson et al., 2005; Rodriguez-Pacheco et al., 2007). It is known that genetic selection for milk yield led to higher circulating GH in dairy cows (Veerkamp et al., 2003). A third hypothesis was that high yielding cows would have lower circulating adiponectin than low yielding cows due to increased circulating $\mathrm{GH}$ and its antagonistic relationship with adiponectin. 


\subsection{MATERIALS AND METHODS}

\subsubsection{Measurement of adiponectin in bovine milk (Experiment 1)}

Milk samples were collected from 6 cows fed on the same diet, but at different stages of lactation (from 5 to 50 days post partum). Cows were milked through a robotic milking system (AMS; Merlin, Fullwood Ltd.). All milk samples were collected between 15:00 and 17:00 in the afternoon and left in the fridge $\left(0\right.$ to $\left.4{ }^{\circ} \mathrm{C}\right)$ overnight. Because lipids interfere with radioimmunoassays (RIA), skim milk was used (Martin et al., 2006). Milk samples $(4 \mathrm{ml})$ were mixed by vortex and skim milk (aqueous phase) was prepared by centrifugation $\left(1500 \times g, 20 \mathrm{~min}, 4^{\circ} \mathrm{C}\right)$ and removal of the fat layer. Adiponectin was determined in duplicate by using a commercial RIA kit (Linco Research, St Charles, MO, USA), as described in the protocol (A.4 in appendix).

\subsubsection{Measurement of circulating adiponectin (Experiment 2)}

\subsubsection{Data}

This study utilized the dataset generated by the study of Garnsworthy et al. (2009). In the current study, circulating adiponectin values were measured and added. Key points of materials and methods, further statistical analysis, and handling of the data are presented hereinafter.

\subsubsection{Experimental design}

Sixty high-yielding multiparous Holstein dairy cows were blocked according to calving date and parity, and were allocated at random to two dietary treatment groups (HS and HF, 30 cows in each). Two diets were formulated to have equal concentrations of DM, ME and CP, but to differ in starch, fat and NDF concentrations (Table A.1 in appendix). Diet HS 
had higher starch content and was expected to induce relatively high plasma insulin concentrations; Diet HF had a higher fat content and was expected to induce relatively low plasma insulin concentrations. These diets were equivalent to the high and low insulin diets used by Gong et al. (2002b), Fouladi-Nashta et al. (2005), and Garnsworthy et al. (2009).

Within dietary treatment groups, cows were allocated on the basis of BCS at calving into FAT (BCS $>3.25$ units) and THIN (BCS $\leq 3.25$ units) cows. The FAT group comprised 40 cows (19 HS; $21 \mathrm{HF}$ ); the THIN group comprised 20 cows (11 HS; 9 HF). Prepartum, all cows were fed on the same diet.

\subsubsection{Feeding and milking}

These are described in $\S 3.2 .3$.

\subsubsection{Recording, sampling and analysis}

Milk yield and feed intake were recorded daily throughout the experiment. Previous milk yield (PMY) was obtained by dairy records while aggregated milk yield (AMY) was calculated by summarizing the milk yield for each cow at the end of the experiment. Live weight and BCS (units; 1 to 5) were recorded weekly. Milk samples were taken twice in a week (for the weeks; $+2,+4,+6,+8,+12,+15$ postpartum) and analyzed for fat, protein and lactose contents by infrared analysis at the National Milk Records Laboratory, Harrogate, UK, using AOAC reference method No. 972.16 (AOAC, 1990). Metabolizable energy balance was calculated for each cow from LWT, milk energy output, and metabolizable energy intake, using UPWin software (AGM Systems, Exeter, UK) and Feed Into Milk equations (Thomas et al., 2004).

Blood samples were taken every Wednesday at 09:30 h from weeks -2 prepartum and +2 , $+4,+6,+8,+12,+15$ postpartum for measurement of hormones and metabolites. Blood 
samples were analyzed for the following hormones: insulin, GH, glucagon, adiponectin, and leptin, as detailed in Garnsworthy et al. (2009). Circulating and milk adiponectin were measured by using a commercially available human adiponectin kit (HADP-61 HK, Linco Research, Millipore, St. Charles, MO, USA) as described in the protocol (A.3 in appendix). The sensitivity of the method (expressed as $\mathrm{ED}_{80}$ ) was $2.33 \mathrm{ng} / \mathrm{ml}$. Blood samples were analyzed for the following metabolites on a Bayer opera autoanalyzer (Bayer UK Ltd): urea-N, glucose, BOHB and NEFA, as detailed in Garnsworthy et al. (2009). All instances of ill health and veterinary treatments were recorded. All analytical methods used to measure metabolites, and metabolic hormones are described in the appendices. 


\subsection{STATISTICAL ANALYSIS}

\subsubsection{Adiponectin in bovine milk (Experiment 1)}

Cows were divided into two groups according to daily milk yield; cows yielding $>30 \mathrm{~kg} / \mathrm{d}$ were the HIGH group ( 3 cows); cows yielding $<30 \mathrm{~kg} / \mathrm{d}$ were the LOW group (3 cows). The difference between groups in milk adiponectin concentration was tested by using Student's t-test within the PASW ${ }^{\mathcal{O}} 18$ Edition (SPSS Inc., Chicago, USA) statistical software package.

\subsubsection{Circulating adiponectin (Experiment 2)}

All data were analyzed using PASW ${ }^{\odot} 18$ Edition (SPSS Inc., Chicago, USA). Generalized Estimating Equations (GEE) were used to test specific hypotheses (Lindsey, 1997; McCullagh \& Nelder, 1989; Dobson, 2002; Horton \& Lipsitz, 1999) because adiponectin values were not normally distributed (Shapiro-Wilk W value $=0.847$, Shapiro-Wilk $P$ value $=0.001$ ), and all known transformations failed to normalize the data. Family distribution and link function were selected as detailed in $\S 3.3$. Selection of GEE models was performed based on QIC criterion, as detailed in $\S 3.3$.

Models selected to test effects of lactational stage (WEEK), BCS at calving (CONDITION) and diet on production traits and hormonal and metabolic profile are illustrated in Table 4.1 and 4.2.

Parameter estimates of GEE models are presented as marginal means plus/minus standard error of the difference (SED) in tables. Estimated marginal means, SEs and SEDs were obtained by using Least Significant Difference ( $L S D)$ adjustment for multiple comparisons in $\mathrm{PASW}^{\odot} 18$ statistical program. $P$ values were calculated by Newton-Raphson Maximum Likelihood $(M L)$ method and effects were considered statistically significant when $P$ value was less than $0.05(P<0.05)$. 
Shapiro-Wilk test was used to assess whether the distribution of variables followed a normal distribution. Linear relationships between quantitative variables were assessed with the Spearman correlation coefficients for nonparametric data. Spearman correlation coefficients ( $r h o$ ) were calculated and considered statistically significant when $P$ value was less than $0.05(P<0.05)$ whereas $P$ value equal or less than $0.1(P \leq 0.1)$ were considered as a trend.

Regression analysis was performed for DMI, adiponectin, leptin, glucose, milk yield, and MEBAL in FAT and THIN cows at calving (Table 4.3). In this way, two regression models for DMI were constructed. The model fitness was inspected by graphing observed values of DMI against predicted values of DMI and R-squared coefficient. Raw residuals and Pearson residuals were calculated, and the validity and the stability of the models were examined by exploring their normality. Beta (unstandardized) coefficients were estimated and they were used to build up regression equations to predict DMI. Beta coefficients were considered statistically significant and entered in the regression equations when $P$ value was less than $0.1(P<0.1)$. 
Table 4.1: Selected models for analysis of the effect of lactational stage on circulating adiponectin, metabolites, metabolic hormones, and production traits

\begin{tabular}{|c|c|c|c|c|c|c|c|c|c|c|}
\hline $\begin{array}{c}\text { RESPONSE } \\
\text { VARIABLES }(Y)\end{array}$ & $\begin{array}{l}\text { TYPE OF } \\
\text { MODEL }\end{array}$ & $\begin{array}{l}\text { PREDICTORS } \\
(X)\end{array}$ & $\begin{array}{l}\text { SUBJECT (ID) } \\
\text { VARIABLE }\end{array}$ & $\begin{array}{l}\text { WITHIN- } \\
\text { SUBJECT } \\
\text { VARIABLE }\end{array}$ & $\begin{array}{l}\text { CONTINUOUS } \\
\text { COVARIATES }\end{array}$ & $\begin{array}{c}\text { ERROR } \\
\text { DISTRIBUTION } \\
\text { FUNCTION }\end{array}$ & $\begin{array}{c}\text { LINK } \\
\text { FUNCTION }\end{array}$ & $\begin{array}{l}\text { COVARIANCE } \\
\text { MATRIX }\end{array}$ & $\begin{array}{l}\text { WORKING } \\
\text { CORRELATION } \\
\text { MATRIX }\end{array}$ & STATISTICS \\
\hline -Adiponectin & GEE & -WEEK & $\begin{array}{l}\text { COW } \\
(60)\end{array}$ & WEEK & PARITY & Gamma & $\log$ & Robust & Exchangeable & $n=378 ; Q \mid C=230$ \\
\hline $\begin{array}{l}\text {-Insulin } \\
\text { - Leptin } \\
\text { - Glucagon } \\
\text { - Glucose } \\
\text {-Urea } \\
\text {-DMI } \\
\text { - Milk yield } \\
\text {-LWT } \\
\text {-BCS } \\
\text {-MEBAL }\end{array}$ & GEE & -WEEK & $\begin{array}{l}\text { COW } \\
(60)\end{array}$ & WEEK & PARITY & $\begin{array}{l}\text { Gaussian } \\
\text { (Normal) }\end{array}$ & $\begin{array}{l}\text { Identity } \\
\text { (ID) }\end{array}$ & Robust & Exchangeable & $\begin{array}{l}n=377 ; Q I C=28 \\
n=378 ; Q I C=474 \\
n=216 ; Q I C=123,651 \\
n=246 ; Q I C=75 \\
n=249 ; Q I C=130 \\
n=352 ; Q \mid C=3,242 \\
n=352 ; Q I C=23,775 \\
n=352 ; Q \mid C=228,742 \\
n=418 ; Q \mid C=79 \\
n=352 ; Q \mid C=50,678\end{array}$ \\
\hline $\begin{array}{l}\bullet \mathrm{GH} \\
\text { •BOHB } \\
\text {-NEFA }\end{array}$ & GEE & $\bullet$ WEEK & $\begin{array}{l}\text { COW } \\
(60)\end{array}$ & WEEK & PARITY & $\begin{array}{l}\text { Inverse } \\
\text { Gaussian }\end{array}$ & $\log$ & Robust & Exchangeable & $\begin{array}{l}n=206 ; Q I C=37 \\
n=248 ; Q I C=122 \\
n=249 ; Q \mid C=224\end{array}$ \\
\hline
\end{tabular}


Table 4.2: Selected models for analysis of the effects of diet and BCS at calving (CONDITION) on circulating adiponectin, metabolites, metabolic hormones, and production traits

\begin{tabular}{|c|c|c|c|c|c|c|c|c|c|c|}
\hline $\begin{array}{c}\text { RESPONSE } \\
\text { VARIABLES }(Y)\end{array}$ & $\begin{array}{l}\text { TYPE OF } \\
\text { MODEL }\end{array}$ & $\begin{array}{l}\text { PREDICTORS } \\
(X)\end{array}$ & $\begin{array}{l}\text { SUBJ. } \\
\text { VAR. }\end{array}$ & $\begin{array}{l}\text { WITHIN- } \\
\text { SUBJECT } \\
\text { VARIABLE }\end{array}$ & $\begin{array}{l}\text { CONTINUOUS } \\
\text { COVARIATES }\end{array}$ & $\begin{array}{c}\text { ERROR } \\
\text { DISTRIBUTION } \\
\text { FUNCTION }\end{array}$ & $\begin{array}{c}\text { LINK } \\
\text { FUNCTION }\end{array}$ & $\begin{array}{l}\text { COVARIANCE } \\
\text { MATRIX }\end{array}$ & $\begin{array}{l}\text { WORKING } \\
\text { CORRELATION } \\
\text { MATRIX }\end{array}$ & STATISTICS \\
\hline -Adiponectin & GEE & $\begin{array}{l}\text {-CONDITION } \\
\text {-DIET* }\end{array}$ & $\begin{array}{l}\text { Cow } \\
(60)\end{array}$ & WEEK & PARITY & Gamma & $\log$ & Robust & Exchangeable & $\mathrm{n}=342 ; \mathrm{QIC}=214$ \\
\hline $\begin{array}{l}\text {-Insulin } \\
\text {-Leptin } \\
\text {-Glucagon } \\
\text {-Glucose } \\
\text {-Urea } \\
\text {-DMI } \\
\text {-Milk yield } \\
\text {-LWT } \\
\text {-BCS } \\
\text {-MEBAL }\end{array}$ & GEE & $\begin{array}{l}\text {-CONDITION } \\
\text {-DIET* }\end{array}$ & $\begin{array}{l}\text { Cow } \\
(60)\end{array}$ & WEEK & PARITY & $\begin{array}{l}\text { Gaussian } \\
\text { (Normal) }\end{array}$ & $\begin{array}{l}\text { Identity } \\
\text { (ID) }\end{array}$ & Robust & Exchangeable & $\begin{array}{l}n=330 ; Q I C=21 \\
n=332 ; Q I C=356 \\
n=216 ; Q I C=121,477 \\
n=202 ; Q I C=69 \\
n=205 ; Q I C=114 \\
n=352 ; Q I C=3,969 \\
n=352 ; Q I C=26,606 \\
n=352 ; Q I C=256,097 \\
n=358 ; Q I C=72 \\
n=352 ; Q \mid C=86,330\end{array}$ \\
\hline$\bullet \triangle B C S$ & GLM & $\begin{array}{l}- \text { CONDITION } \\
\text {-DIET }\end{array}$ & - & - & PARITY & $\begin{array}{l}\text { Gaussian } \\
\text { (Normal) }\end{array}$ & $\begin{array}{l}\text { Identity } \\
\text { (ID) }\end{array}$ & Robust & Independent & $\begin{array}{l}\mathrm{n}=59 ; \mathrm{AIC}=77 ; \quad L R( \\
\left.\chi^{2}=8.81, \mathrm{df}=2, P=0.032\right)\end{array}$ \\
\hline $\begin{array}{l}\text {-GH } \\
\text {-BOHB } \\
\text {-NEFA }\end{array}$ & GEE & $\begin{array}{l}\text {-CONDITION } \\
\text {-DIET* }\end{array}$ & $\begin{array}{l}\text { Cow } \\
(60)\end{array}$ & WEEK & PARITY & $\begin{array}{l}\text { Inverse } \\
\text { Gaussian }\end{array}$ & $\log$ & Robust & Exchangeable & $\begin{array}{l}n=206 ; Q I C=41 \\
n=205 ; Q I C=93 \\
n=205 ; Q I C=273\end{array}$ \\
\hline
\end{tabular}

* Precalving data were excluded from the analysis 
Table 4.3: Selected model for regression analysis of DMI

\begin{tabular}{|c|c|c|c|c|c|c|c|c|c|c|}
\hline $\begin{array}{c}\text { RESPONSE } \\
\text { VARIABLES }(Y)\end{array}$ & $\begin{array}{c}\text { TYPE } \\
\text { OF } \\
\text { MODEL }\end{array}$ & $\begin{array}{l}\text { PREDICTORS } \\
(X)\end{array}$ & $\begin{array}{l}\text { SUBJECT } \\
\text { (ID) } \\
\text { VARIABLE }\end{array}$ & $\begin{array}{l}\text { WITHIN- } \\
\text { SUBJECT } \\
\text { VARIABLE }\end{array}$ & $\begin{array}{l}\text { CONTINUOUS } \\
\text { COVARIATES }\end{array}$ & $\begin{array}{c}\text { ERROR } \\
\text { DISTRIBUTION } \\
\text { FUNCTION }\end{array}$ & $\begin{array}{c}\text { LINK } \\
\text { FUNCTION }\end{array}$ & $\begin{array}{l}\text { COVARIANCE } \\
\text { MATRIX }\end{array}$ & $\begin{array}{c}\text { WORKING } \\
\text { CORRELATION } \\
\text { MATRIX }\end{array}$ & OBSERVATIONS \\
\hline ODMI* & GEE & - & $\begin{array}{c}\text { COW } \\
(60 \text { cows })\end{array}$ & WEEK & $\begin{array}{l}\text { Adiponectin } \\
\text { Milk yield } \\
\text { Glucose } \\
\text { Leptin } \\
\text { MEBAL }\end{array}$ & $\begin{array}{l}\text { Gaussian } \\
\text { (Normal) }\end{array}$ & $\begin{array}{l}\text { Identity } \\
\text { (ID) }\end{array}$ & $\begin{array}{c}\text { Robust } \\
\text { Estimator }\end{array}$ & Exchangeable & $\begin{array}{c}111 \\
\text { (each time)* }^{*}\end{array}$ \\
\hline
\end{tabular}

*The same model was run twice, for FAT and THIN cows at calving. 


\subsection{RESULTS}

\subsubsection{Adiponectin in bovine milk (Experiment 1)}

Mean milk adiponectin concentration was $11.5 \pm 3.49 \mathrm{ng} / \mathrm{ml}$. Milk adiponectin varied with day in milk, from low ( $<5 \mathrm{ng} / \mathrm{ml} ; 10$ and 46 day in milk) to moderate $(10 \mathrm{ng} / \mathrm{ml} ; 6$ day in milk) and high (20-25 $\mathrm{ng} / \mathrm{ml} ; 15-36$ day in milk). Cows with HIGH daily milk yield did not have higher $(P=0.31)$ milk adiponectin $(8.8 \pm 4.01 \mathrm{ng} / \mathrm{ml}$ versus $17.0 \pm 6.40 \mathrm{ng} / \mathrm{ml})$ than cows with LOW daily milk yield.

\subsubsection{Circulating adiponectin (Experiment 2)}

Mean circulating adiponectin was $8.6 \pm 0.33 \mathrm{ng} / \mathrm{ml}$ and this was greater than the mean of other metabolic hormone (insulin; $0.4 \pm 0.01 \mathrm{ng} / \mathrm{ml}$, leptin; $1.7 \pm 0.06 \mathrm{ng} / \mathrm{ml}, \mathrm{GH} ; 4.6 \pm 0.22$ $n g / m l$, glucagon; $95.9 \pm 0.63 \mathrm{pg} / \mathrm{ml})$. Circulating adiponectin values showed high correlation (average correlation; $\rho=0.844$ ) by week of experiment. They were not normally distributed (Shapiro-Wilk $W$ value $=0.847$, Shapiro-Wilk $P$ value=0.001), left skewed (median=7.21; skewness $=1.80$ ), and highly peaked (leptokurtotic probability distribution; kurtosis=7.49). Individual cows showed consistently high or normal circulating adiponectin values throughout the experimental period (Figure 4.1).

\subsubsection{Lactational stage}

There was an effect of lactational stage on circulating adiponectin, insulin, leptin, and GH $(P<0.01)$ (Table 4.4). Circulating adiponectin was elevated 2 weeks prepartum $(11.1 \pm 1.06$ $n g / m l)$, decreased to week 2 postpartum $(7.1 \pm 0.90 \mathrm{ng} / \mathrm{ml})$, increased to week 4 postpartum $(8.9 \pm 0.76 \mathrm{ng} / \mathrm{ml})$, remained stable until week 12 postpartum $(9.1 \pm 0.86 \mathrm{ng} / \mathrm{ml})$, and then declined to $6.5 \pm 0.75 \mathrm{ng} / \mathrm{ml}$ at the end of the study (Figure 4.2). Circulating insulin 
decreased from week 2 prepartum $(0.4 \pm 0.03 n g / m l)$ to week 2 postpartum $(0.3 \pm 0.02$ $n g / m l$ ), and then increased constantly between 0.33 and $0.54 \mathrm{ng} / \mathrm{ml}$ for the remainder of the study. Circulating leptin was elevated 2 weeks prepartum $(2.2 \pm 0.24 \mathrm{ng} / \mathrm{ml})$, decreased until week 2 postpartum $(1.5 \pm 0.10 \mathrm{ng} / \mathrm{ml})$, remained at $1.4-1.64 \mathrm{ng} / \mathrm{ml}$ until the end of week 8 postpartum, and then increased constantly for the remainder of the study (Table 4.4). Circulating GH was elevated 2 weeks postpartum $(6.9 \pm 0.53 \mathrm{ng} / \mathrm{ml})$, decreased steadily until week $12(3.2 \pm 0.53 \mathrm{ng} / \mathrm{ml})$, and remained unchanged after week 12 postpartum (Table 4.4). There was no effect of lactational stage on circulating glucagon (Table 4.4).

There was an effect of lactational stage on circulating glucose, NEFA, and urea $(P<0.01)$ (Table 4.5). Circulating glucose was $(3.0 \pm 0.07 \mathrm{mmol} / \mathrm{l})$ in week 2 prepartum and week 2 postpartum, increased rapidly to week 8 postpartum $(3.6 \pm 0.08)$, and then remained at the same level until the end of the experiment (Table 4.5). NEFA increased from 2 weeks prepartum $(0.4 \pm 0.05 \mathrm{mmol} / \mathrm{l})$ to week 2 postpartum $(0.9 \pm 0.16 \mathrm{mmol} / \mathrm{l})$, then declined substantially and remained between 0.54 and $0.24 \mathrm{mmol} / \mathrm{l}$ for the remainder of the study (Table 4.5). Circulating urea was lowest 2 weeks prepartum $(1.6 \pm 0.10 \mathrm{mmol} / \mathrm{l})$ and increased steadily throughout the study. There was no effect of lactational stage on circulating BOHB (Table 4.5).

There was an effect of lactational stage on DMI, LWT, milk yield, BCS, and MEBAL $(P<0.01)$ (Table 4.6). DMI $(17.9 \pm 0.23 \mathrm{~kg} / \mathrm{d})$ and milk yield $(35.1 \pm 0.52 \mathrm{~kg} / \mathrm{d})$ were low 2 weeks postpartum, increased until week 8 postpartum (DMI $22.8 \pm 0.23 \mathrm{~kg} / \mathrm{d}$; milk yield $46.8 \pm 0.52 \mathrm{~kg} / \mathrm{d}$ ) and decreased after this point to be $22.3 \pm 0.23 \mathrm{~kg} / \mathrm{d}$ and $41.1 \pm 0.51 \mathrm{~kg} / \mathrm{d}$ respectively, at the end of the study (Table 4.6). BCS was high 2 weeks postpartum (2.9 \pm 0.04$)$ and decreased rapidly until week 6 postpartum (2.4 \pm 0.04$)$. After week 6 postpartum, cows gained condition for the remainder of the study (Table 4.6). Metabolizable energy balance (MEBAL) was negative from week 2 to week 6 postpartum and remained positive after week 8. LWT remained between 650 and $654 \mathrm{~kg}$ from week 2 to week 6 postpartum and then increased steadily to reach $669 \mathrm{~kg}$ at the end of the study (Table 4.6). 


\subsubsection{Diet and BCS at calving}

Circulating adiponectin was not different $(P>0.05)$ for cows fed the HS diet $(8.3 \pm 0.68$ $n g / m l)$ than for cows fed the HF diet $(8.8 \pm 0.81 n g / m l)$. Cows fed the HS diet had higher circulating insulin, NEFA, and BOHB $(P<0.05)$ than cows fed the HF diet (Tables $4.7 \&$ 4.8). There was no effect of DIET on DMI, LWT, milk yield, BCS, $\triangle B C S$, and MEBAL (Table 4.9).

Circulating adiponectin was not different $(P>0.05)$ for FAT cows at calving $(8.3 \pm 0.87$ $n g / m l)$ than for THIN cows at calving $(8.91 .39 n g / m l)$ (Table 4.7). There was no effect of BCS at calving on circulating insulin, leptin, GH, glucagon, glucose, NEFA, BOHB, and urea (Tables $4.7 \& 4.8$ ). FAT cows at calving had higher DMI, milk yield, BCS, and $\triangle \mathrm{BCS}$ than THIN cows at calving $(P<0.05)$. There was no effect of BCS at calving on LWT and MEBAL (Table 4.9).

\subsubsection{Associations of circulating adiponectin with circulating metabolites, metabolic hormones, and production traits}

Plasma adiponectin was negatively correlated with $\mathrm{GH}$ concentration ( $r h o=-0.144$, $P=0.042)$ and tended to be correlated negatively with plasma glucose ( $r h o=-0.110$, $P=0.10$ ). Circulating $\mathrm{GH}$ was correlated negatively with circulating insulin ( $r h o=-0.287$, $P=0.001)$ and leptin ( $r h o=-0.319, P=0.001)$. Circulating insulin was correlated negatively with $\triangle \mathrm{BCS}(r h o=-0.158, P=0.002)$

Circulating adiponectin was correlated negatively with previous milk yield (PMY; $r h o=-$ 0.256, $P=0.001$ ) and aggregated milk yield (AMY; $r h o=-0.119, P=0.02$ ). AMY was correlated positively with PMY ( $r h o=0.430, P=0.001)$. GH was correlated positively with AMY $(r h o=0.216, P=0.002)$ and PMY $(r h o=0.127, P=0.05)$. Leptin was correlated negatively with PMY ( $r h o=-0.122, P=0.018)$ and positively with circulating insulin $(r h o=$ $0.356, P=0.001$ ). 
Adiponectin concentration was correlated negatively with $\triangle \mathrm{BCS}(r h o=-0.173, P=0.001)$. $\triangle \mathrm{BCS}$ was correlated positively with $\mathrm{GH}(r h o=0.253, P=0.001)$, and negatively with circulating leptin $(r h o=-0.261, P=0.001) . \triangle \mathrm{BCS}$ was correlated positively with PMY $(r h o=0.281, P=0.001)$, AMY $(r h o=0.434, P=0.001)$, milk yield $(r h o=0.388, P=0.001)$, DMI $(r h o=0.382, P=0.001)$ and BOHB $(r h o=-0.193, P=0.002)$.

Milk yield was correlated positively with PMY $(r h o=0.473, P=0.001)$ and AMY $(r h o=$ 0.753, $P=0.001)$. AMY was correlated positively with PMY $(r h o=0.513, P=0.001)$ and DMI $(r h o=0.595, P=0.001)$. PMY was correlated positively with DMI $(r h o=0.365$, $P=0.001)$.

\subsubsection{Relationships between DMI and circulating adiponectin, glucose, leptin, MEBAL, and milk yield in FAT and THIN cows at calving}

DMI for THIN cows at calving can be predicted, with high precision $\left(\mathrm{R}^{2}=0.88\right)$, by the regression equation; $\mathrm{DMI}=0.318 * \mathrm{MY}(\mathrm{kg} / \mathrm{d})+0.057 * \mathrm{MEBAL}(\mathrm{MJ} / \mathrm{d})-0.043 * \mathrm{ADIPO}$ $(n g / m l)+8.74$, whereas DMI for FAT cows at calving can be predicted by the regression equation; DMI $=0.293 * \mathrm{MY}(\mathrm{kg} / \mathrm{d})+0.055 * \operatorname{MEBAL}(\mathrm{MJ} / \mathrm{d})+0.047 * \mathrm{ADIPO}(\mathrm{ng} / \mathrm{ml})-$ 0.54* GLU $(m m o l / l)+10.71\left(\mathrm{R}^{2}=0.78\right)$ (Figure 4.3). Adiponectin beta coefficient in the regression equation for THIN cows was negative $(-0.043)$, whereas it was positive $(0.047)$ for FAT cows at calving (Table $4.10 \& 4.11$ ). Glucose featured only in the model for FAT cows at calving and its beta coefficient was negative (-0.54) (Table 4.11). 


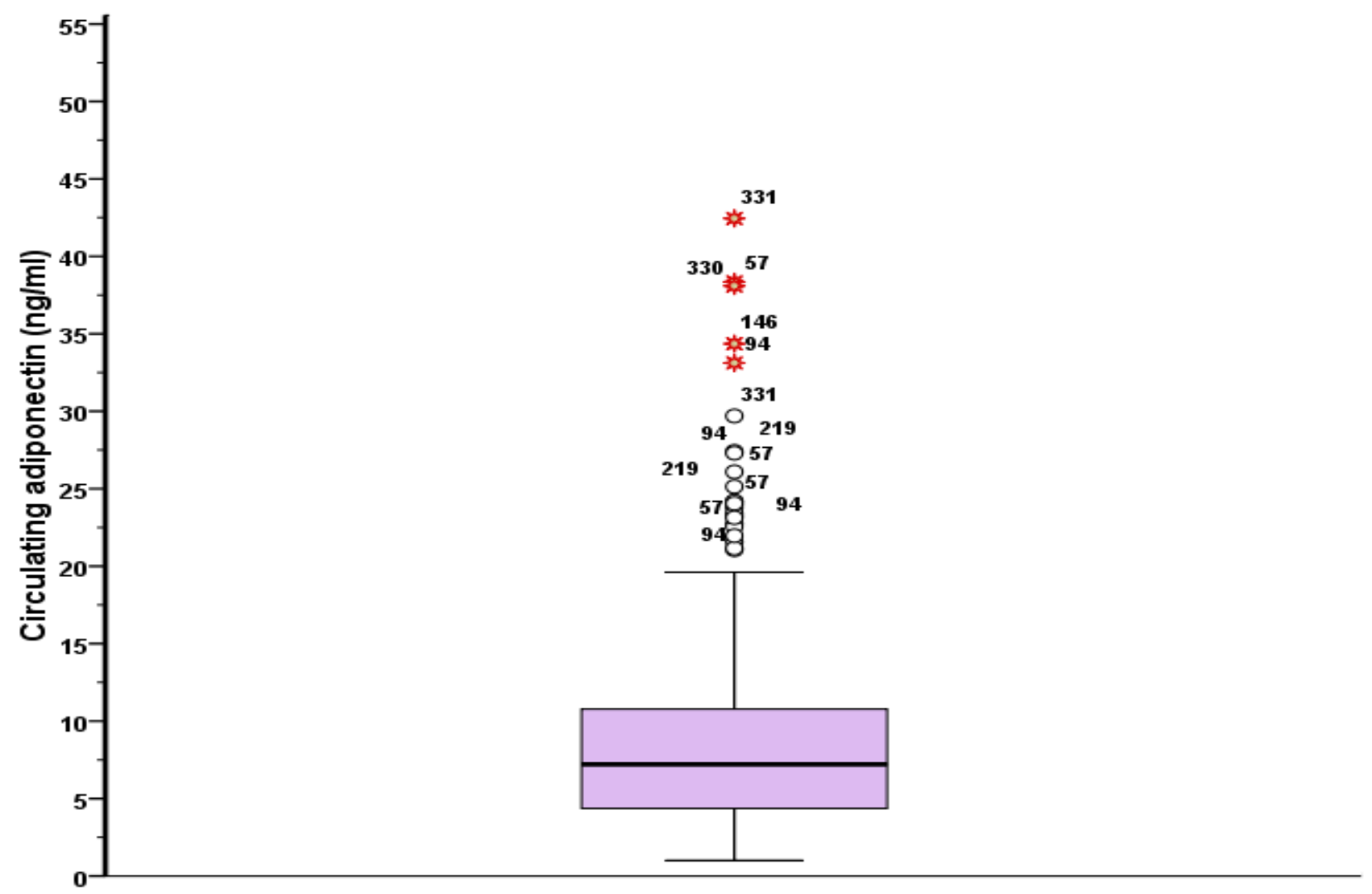

Figure 4.1: Box - Whisker plot of circulating adiponectin values $(\boldsymbol{n}=\mathbf{3 8 7})$. Box shows median value of circulating adiponectin, and the size of the first and third quartile. Outliers appear as individual points $O$ or $*$ outside the box (marked by cow id number). The $O$ outlier values are known as outside values, and the $*$ outlier values as far outside values. Outliers are at the upper range of the data (above the box); the mean value is above the median (the centre line in the box); the median line does not evenly divide the box; and the upper tail of the box plot is longer than the lower tail; thus, the population distribution from which the data were sampled is skewed to the right. Interestingly, outlier values come consistently from 6 cows (id; 57, 94, 146, 219, 330, and 331) which showed the same trend for higher adiponectin values throughout the experiment. 


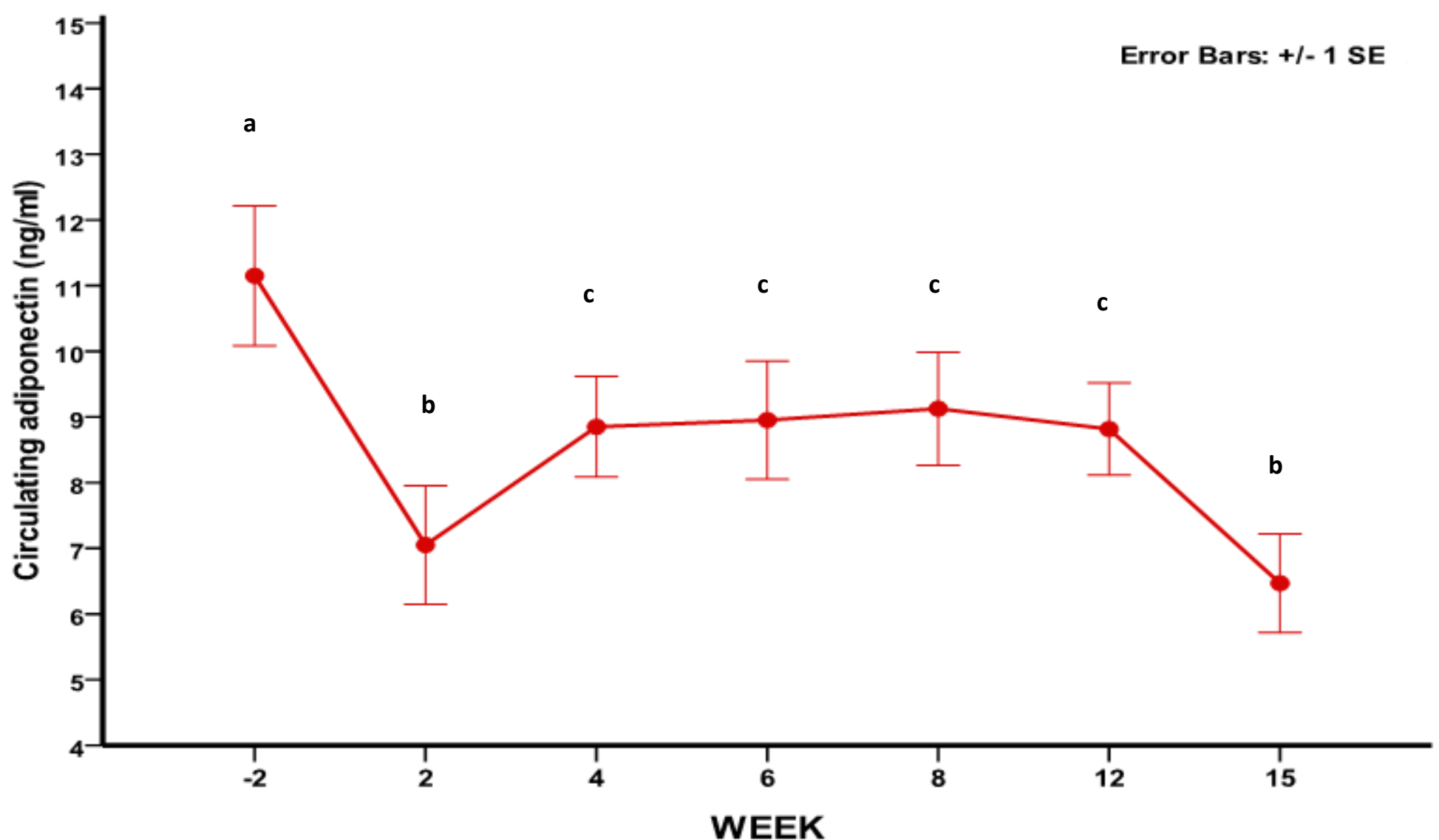

Figure 4.2: Effect of lactational stage (WEEK) on circulating adiponectin $(n=387, \mathrm{P}$ value $=0.001)$. GEE model with Gamma error distribution, $\log$ link function, and exchangeable $R$ matrix structure with robust estimator of covariance matrix. Dependent variable was adiponectin, subject variable was cows $(60$ cows), and within subject variable was WEEK ( 7 weeks). WEEK was added as factorial independent, and parity as continuous covariate in the model). Red dots $(\bullet)$ represent marginal means \pm SE. -2 point in $x$-axis represents two weeks prepartum whereas 15 is fifteen week postpartum. Means marked with different letters are significant different $(P<0.05)$. 
Table 4.4: Effect of lactational stage (WEEK) on circulating metabolic hormones

\begin{tabular}{|c|c|c|c|c|c|c|c|c|c|c|c|c|c|c|c|}
\hline \multirow{2}{*}{$\begin{array}{c}\text { Factors: } \\
\text { Parameters: }\end{array}$} & \multicolumn{7}{|c|}{ WEEK } & \multirow[b]{2}{*}{ SED } & \multirow[b]{2}{*}{$n^{\partial}$} & \multicolumn{6}{|c|}{ Contrasts $^{\diamond} P$ val. } \\
\hline & $-2^{\dagger}$ & $2^{\dagger}$ & $4^{\dagger}$ & $6^{\dagger}$ & $\mathbf{8}^{\dagger}$ & $12^{\dagger}$ & $15^{\dagger}$ & & & $c 1$ & $c 2$ & $c 3$ & $c 4$ & $c 5$ & $c 6$ \\
\hline $\begin{array}{l}\text { Adiponectin } \\
\quad(n g / m l)\end{array}$ & 11.19 & 7.01 & 8.83 & 8.90 & 9.09 & 8.82 & 6.48 & 0.842 & 387 & $<0.01$ & $<0.01$ & $\mathrm{NS}^{*}$ & NS & NS & $<0.01$ \\
\hline $\begin{array}{c}\text { Insulin } \\
(n g / m l)\end{array}$ & 0.36 & 0.26 & 0.33 & 0.40 & 0.45 & 0.48 & 0.54 & 0.026 & 377 & $<0.01$ & NS & $<0.01$ & $<0.01$ & $<0.01$ & $<0.01$ \\
\hline $\begin{array}{c}\mathbf{G H} \\
(n g / m l)\end{array}$ & - & 6.9 & 5.1 & 4.1 & 3.9 & 3.2 & 3.7 & 0.53 & 206 & - & $<0.01$ & $<0.01$ & $<0.01$ & $<0.01$ & NS \\
\hline $\begin{array}{l}\text { Leptin } \\
(n g / m l)\end{array}$ & 2.21 & 1.46 & 1.43 & 1.50 & 1.65 & 1.84 & 2.15 & 0.110 & 378 & $<0.01$ & NS & NS & $<0.05$ & NS & $<0.05$ \\
\hline $\begin{array}{c}\text { Glucagon } \\
(p g / m l)\end{array}$ & - & 96.3 & 93.5 & 91.7 & 92.1 & 96.5 & 100.4 & 4.30 & 216 & $\mathrm{NS}$ & NS & $\mathrm{NS}$ & $\mathrm{NS}$ & NS & NS \\
\hline
\end{tabular}

Columns are means.

* SED = standard error of the difference between treatment means.

${ }^{\diamond}$ c1 (-2 week versus 2 week); c2 (2 week versus 4 week); c3 (4 week versus 6 week); c4 (6 week versus 8 week); c5 (8 week versus 12 week); c6 (12 week versus 15 week).

${ }^{\partial} n$ is the total repeated observations of animals

*NS = non-significant 
Table 4.5: Effect of lactational stage (WEEK) on circulating metabolites

\begin{tabular}{|c|c|c|c|c|c|c|c|c|c|c|c|c|c|c|c|}
\hline \multirow{2}{*}{$\begin{array}{c}\text { Factors: } \\
\text { Parameters: }\end{array}$} & \multicolumn{7}{|c|}{ WEEK } & \multirow[b]{2}{*}{ SED } & \multirow[b]{2}{*}{$n^{\partial}$} & \multicolumn{6}{|c|}{ Contrasts $^{\diamond} P$ val. } \\
\hline & $-2^{\dagger}$ & $2^{\dagger}$ & $4^{\dagger}$ & $6^{\dagger}$ & $\mathbf{8}^{\dagger}$ & $12^{\dagger}$ & $15^{\dagger}$ & & & $c 1$ & $c 2$ & $c 3$ & $c 4$ & $c 5$ & $c 6$ \\
\hline $\begin{array}{l}\text { Glucose } \\
(\mathrm{mmol} / \mathrm{l})\end{array}$ & 3.03 & 3.07 & 3.27 & 3.44 & 3.60 & 3.74 & 3.75 & 0.090 & 246 & $\mathrm{NS}^{*}$ & $<0.05$ & $<0.05$ & $<0.05$ & NS & NS \\
\hline $\begin{array}{c}\text { ВОНB } \\
(\mathrm{mmol} / \mathrm{l})\end{array}$ & 0.70 & 0.73 & 0.68 & 0.59 & 0.60 & 0.61 & 0.62 & 0.074 & 248 & NS & NS & $\mathrm{NS}$ & $\mathrm{NS}$ & NS & NS \\
\hline $\begin{array}{c}\text { NEFA } \\
(\mathrm{mmol} / \mathrm{l})\end{array}$ & 0.45 & 0.88 & 0.54 & 0.47 & 0.28 & 0.24 & 0.23 & 0.085 & 249 & $<0.01$ & $<0.05$ & NS & $<0.05$ & NS & NS \\
\hline $\begin{array}{c}\text { Urea } \\
(\mathrm{mmol} / \mathrm{l})\end{array}$ & 1.63 & 2.11 & 2.26 & 2.51 & 2.67 & 2.71 & 2.90 & 0.124 & 249 & $<0.01$ & $\mathrm{NS}$ & $<0.05$ & $\mathrm{NS}$ & NS & NS \\
\hline $\begin{array}{l}\text { Columns are mear } \\
\text { SED = standard e } \\
\text { c1 (-2 week versu } \\
\text { veek). } \\
n \text { is the total repe } \\
\text { NS }=\text { non-significa }\end{array}$ & $\begin{array}{l}\text { of the c } \\
\text { eek); } c^{2} \\
\text { observ }\end{array}$ & $\begin{array}{l}\text { ifferenc } \\
(2 \text { week } \\
\text { tions of }\end{array}$ & $\begin{array}{l}\text { netwe } \\
\text { ersus } \\
\text { nimal }\end{array}$ & veek) & $(4 \mathrm{w}$ & versus & veek); & $4(6 \mathrm{we}$ & ersu & week); & (8 week & rsus 12 & k); c6 ( & eek & s 15 \\
\hline
\end{tabular}


Table 4.6: Effect of lactational stage (WEEK) on productive traits

\begin{tabular}{|c|c|c|c|c|c|c|c|c|c|c|c|c|c|}
\hline \multirow{2}{*}{$\begin{array}{c}\text { Factors: } \\
\text { Parameters: }\end{array}$} & \multicolumn{6}{|c|}{ WEEK } & \multirow[b]{2}{*}{ SED } & \multirow[b]{2}{*}{$n^{\partial}$} & \multicolumn{5}{|c|}{ Contrasts $^{\diamond} P$ val. } \\
\hline & $2^{\dagger}$ & $\mathbf{4}^{\dagger}$ & $6^{\dagger}$ & $\mathbf{8}^{\dagger}$ & $12^{\dagger}$ & 15 & & & $c 2$ & $c 3$ & $c 4$ & $c 5$ & $c 6$ \\
\hline $\begin{array}{l}\text { DMI } \\
(k g / d)\end{array}$ & 17.9 & 20.8 & 22.2 & 22.8 & 22.7 & 22.3 & 0.23 & 352 & $<0.01$ & $<0.01$ & $<0.01$ & NS & NS \\
\hline $\begin{array}{c}\text { LWT } \\
(k g)\end{array}$ & 654.7 & 645.2 & 650.0 & 657.4 & 667.6 & 669.3 & 3.00 & 352 & $<0.01$ & $\mathrm{NS}^{*}$ & $<0.01$ & $<0.01$ & $\mathrm{NS}$ \\
\hline $\begin{array}{l}\text { Milk yield } \\
\qquad(\mathrm{kg} / \mathrm{d})\end{array}$ & 35.1 & 43.2 & 45.9 & 46.9 & 45.6 & 44.1 & 0.51 & 352 & $<0.01$ & $<0.01$ & $<0.05$ & $<0.05$ & $<0.01$ \\
\hline $\begin{array}{c}\text { BCS } \\
\text { (units 1-5) }\end{array}$ & 2.95 & 2.60 & 2.45 & 2.51 & 2.64 & 2.77 & 0.037 & 418 & $<0.01$ & $<0.01$ & $<0.05$ & $<0.01$ & $<0.01$ \\
\hline $\begin{array}{c}\text { MEBAL } \\
(M J / d)\end{array}$ & -24.3 & -12.6 & -5.4 & 1.1 & 5.7 & 2.7 & 1.70 & 352 & $<0.01$ & $<0.01$ & $<0.01$ & $<0.05$ & NS \\
\hline
\end{tabular}

${ }^{\dagger}$ Columns are means.

${ }^{*}$ SED $=$ standard error of the difference between treatment means.

$\checkmark$ Contrasts: c2 (2 week versus 4 week); c3 (4 week versus 6 week); c4 (6 week versus 8 week); c5 (8 week versus 12 week); c6 (12 week versus 15 week).

${ }^{\partial} n$ is the total repeated observations of animals

* NS = non-significant 
Table 4.7: Effect of diet and BCS at calving (CONDITION) on circulating metabolic hormones

\begin{tabular}{|c|c|c|c|c|c|c|c|c|c|c|c|c|}
\hline \multirow{2}{*}{$\begin{array}{c}\text { Factors: } \\
\text { Parameters: }\end{array}$} & \multicolumn{5}{|c|}{ DIET } & \multirow[b]{2}{*}{$P$} & \multicolumn{5}{|c|}{ CONDITION } & \multirow[b]{2}{*}{$P$} \\
\hline & $\mathbf{H S}^{\dagger}$ & $n_{1}^{\diamond}$ & $\mathbf{H F}^{\dagger}$ & $\boldsymbol{n}_{2} \diamond$ & SED & & $\mathbf{F A T}^{\dagger}$ & $n_{3}{ }^{\partial}$ & THIN $^{\dagger}$ & $n_{4}{ }^{\partial}$ & SED & \\
\hline $\begin{array}{l}\text { Adiponectin } \\
(\mathrm{ng} / \mathrm{ml})\end{array}$ & 8.29 & 170 & 8.77 & 172 & 0.745 & 0.23 & 8.34 & 229 & 8.92 & 113 & 1.130 & 0.73 \\
\hline $\begin{array}{l}\text { Insulin } \\
(\mathrm{ng} / \mathrm{ml})\end{array}$ & 0.46 & 168 & 0.38 & 162 & 0.027 & 0.01 & 0.40 & 223 & 0.43 & 107 & 0.029 & 0.37 \\
\hline $\begin{array}{c}\mathbf{G H} \\
(n g / m l)\end{array}$ & 4.7 & 94 & 4.2 & 112 & 0.32 & 0.31 & 4.7 & 126 & 4.2 & 80 & 0.54 & 0.30 \\
\hline $\begin{array}{l}\text { Leptin } \\
(n g / m l)\end{array}$ & 1.75 & 165 & 1.67 & 167 & 0.183 & 0.66 & 1.59 & 224 & 1.82 & 108 & 0.265 & 0.41 \\
\hline $\begin{array}{c}\text { Glucagon } \\
(\mathrm{pg} / \mathrm{ml})\end{array}$ & 96.6 & 105 & 93.0 & 111 & 2.61 & 0.16 & 96.8 & 140 & 92.8 & 76 & 5.41 & 0.46 \\
\hline
\end{tabular}

${ }^{\dagger}$ Means for high-starch (HS) and high-fat $($ HF) diet groups, and FAT $(>3.25)$ and THIN $(\leq 3.25)$ BCS at calving groups.

$\diamond n_{1}$ and $n_{2}$ are the repeated observations of animals fed with HS and HF diets, respectively.

$n_{3}$ and $n_{4}$ are the repeated observations of animals assessed FAT and THIN at calving, respectively.

${ }^{\star}$ SED $=$ standard error of the difference between treatment means. 
Table 4.8: Effect of diet and BCS at calving (CONDITION) on circulating metabolites

\begin{tabular}{|c|c|c|c|c|c|c|c|c|c|c|c|c|}
\hline \multirow{2}{*}{$\begin{array}{c}\text { Factors: } \\
\text { Parameters: }\end{array}$} & \multicolumn{5}{|c|}{ DIET } & \multirow[b]{2}{*}{$\boldsymbol{P}$} & \multicolumn{5}{|c|}{ CONDITION } & \multirow[b]{2}{*}{$\boldsymbol{P}$} \\
\hline & $\mathbf{H S}^{\dagger}$ & $n_{1}^{\diamond}$ & $\mathbf{H F}^{\dagger}$ & $\boldsymbol{n}_{2} \diamond$ & SED & & $\mathbf{F A T}^{\dagger}$ & $n_{3}^{\partial}$ & THIN $^{\dagger}$ & $n_{4}^{\partial}$ & SED & \\
\hline $\begin{array}{l}\text { Glucose } \\
(\mathrm{mmol} / \mathrm{l})\end{array}$ & 3.56 & 98 & 3.44 & 104 & 0.096 & 0.20 & 3.44 & 117 & 3.56 & 85 & 0.113 & 0.28 \\
\hline $\begin{array}{c}\text { BOHB } \\
(\mathrm{mmol} / \mathrm{l})\end{array}$ & 0.54 & 98 & 0.70 & 107 & 0.051 & 0.002 & 0.66 & 120 & 0.58 & 85 & 0.052 & 0.13 \\
\hline $\begin{array}{c}\text { NEFA } \\
(\mathrm{mmol} / \mathrm{l})\end{array}$ & 0.36 & 98 & 0.49 & 107 & 0.065 & 0.044 & 0.48 & 123 & 0.37 & 82 & 0.066 & 0.08 \\
\hline $\begin{array}{c}\text { Urea } \\
(\mathrm{mmol} / \mathrm{l})\end{array}$ & 2.65 & 96 & 2.41 & 109 & 0.119 & 0.047 & 2.63 & 121 & 2.44 & 84 & 0.163 & 0.23 \\
\hline
\end{tabular}

${ }^{\dagger}$ Means for high-starch (HS) and high-fat (HF) diet groups, and FAT $(>3.25)$ and THIN $(\leq 3.25)$ BCS at calving groups.

$\diamond \boldsymbol{n}_{1}$ and $\boldsymbol{n}_{2}$ are the repeated observations of animals fed with HS and HF diets, respectively.

$n_{3}$ and $n_{4}$ are the repeated observations of animals assessed FAT and THIN at calving, respectively.

${ }^{*} \mathrm{SED}=$ standard error of the difference between treatment means. 
Table 4.9: Effect of diet and BCS at calving (CONDITION) on production traits

\begin{tabular}{|c|c|c|c|c|c|c|c|c|c|c|c|c|}
\hline \multirow{2}{*}{$\begin{array}{c}\text { Factors: } \\
\text { Parameters: }\end{array}$} & \multicolumn{5}{|c|}{ DIET } & \multirow[b]{2}{*}{$P$} & \multicolumn{5}{|c|}{ CONDITION } & \multirow[b]{2}{*}{$P$} \\
\hline & $\mathbf{H} \mathbf{S}^{\dagger}$ & $n_{1}^{\diamond}$ & $\mathbf{H F}^{\dagger}$ & $\boldsymbol{n}_{2} \diamond$ & SED & & $\mathbf{F A T}^{\dagger}$ & $n_{3}{ }^{\partial}$ & THIN $^{\dagger}$ & $n_{4}^{\partial}$ & SED & \\
\hline $\begin{array}{l}\text { DMI } \\
(\mathrm{kg} / \mathrm{d})\end{array}$ & 21.0 & 176 & 21.4 & 176 & 0.35 & 0.23 & 22.1 & 237 & 20.3 & 115 & 0.72 & 0.02 \\
\hline $\begin{array}{c}\text { LWT } \\
(\mathrm{kg})\end{array}$ & 657.7 & 176 & 657.6 & 176 & 4.21 & 0.99 & 656.5 & 237 & 658.2 & 115 & 5.53 & 0.68 \\
\hline $\begin{array}{l}\text { Milk yield } \\
\quad(\mathrm{kg} / \mathrm{d})\end{array}$ & 42.8 & 176 & 42.5 & 176 & 0.80 & 0.75 & 45.4 & 237 & 39.9 & 115 & 1.99 & 0.01 \\
\hline $\begin{array}{c}\text { BCS } \\
\text { (units 1-5) }\end{array}$ & 2.61 & 180 & 2.60 & 178 & 0.046 & 0.92 & 2.75 & 240 & 2.47 & 118 & 0.075 & 0.01 \\
\hline $\begin{array}{c}\Delta \mathbf{B C S} \\
\text { (units 1-5) }\end{array}$ & 0.60 & 30 & 0.58 & 29 & 0.083 & 0.81 & 0.77 & 40 & 0.41 & 19 & 0.122 & 0.01 \\
\hline $\begin{array}{c}\text { MEBAL } \\
(M J / d)\end{array}$ & -4.7 & 176 & -6.2 & 176 & 2.05 & 0.46 & -5.6 & 237 & -5.3 & 115 & 1.59 & 0.87 \\
\hline
\end{tabular}

${ }^{\dagger}$ Means for high-starch (HS) and high-fat (HF) diet groups, and FAT $(>3.25)$ and THIN $(\leq 3.25)$ BCS at calving groups.

$\diamond n_{1}$ and $n_{2}$ are the repeated observations of animals fed with HS and HF diets, respectively.

$n_{3}$ and $n_{4}$ are the repeated observations of animals assessed FAT and THIN at calving, respectively.

${ }^{\ddagger}$ SED $=$ standard error of the difference between treatment means. 


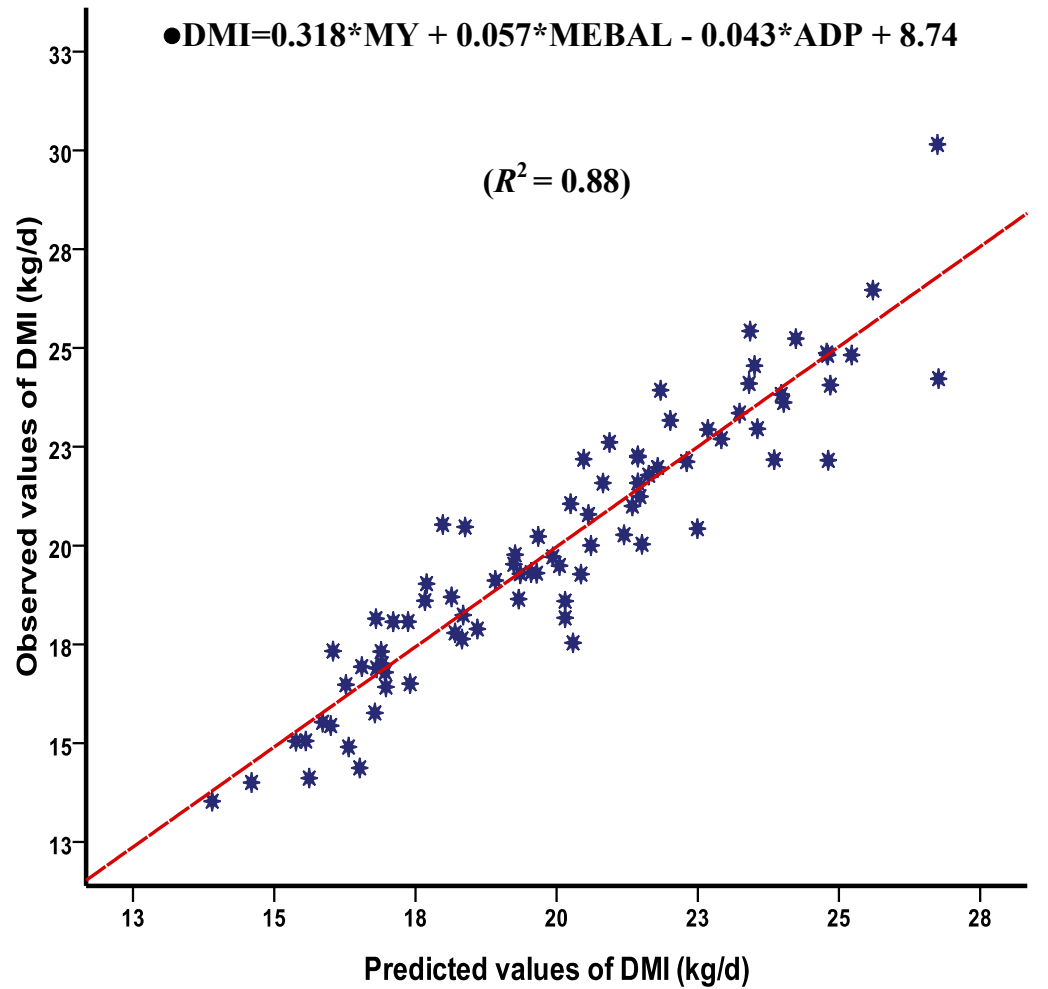

(A) THIN cows at calving

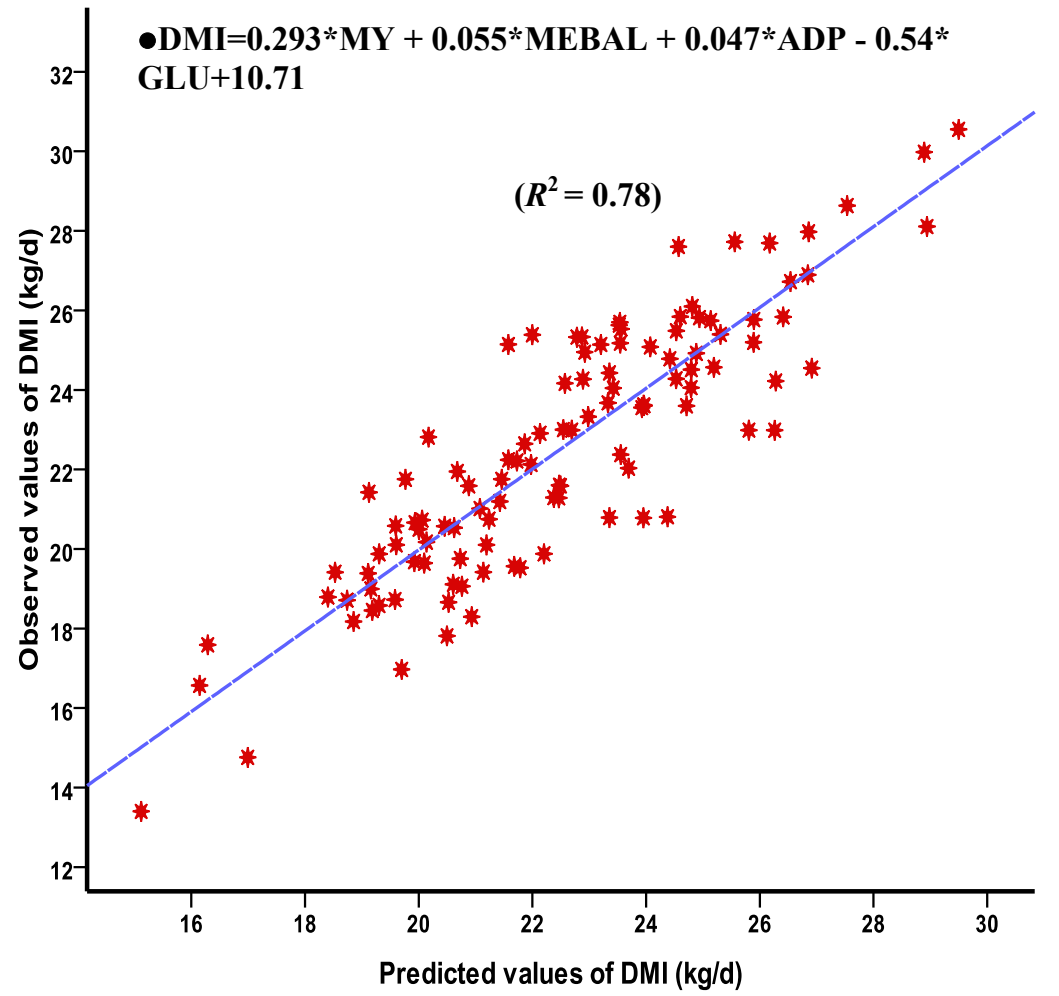

(B) FAT cows at calving

Figure 4.3: Relationships among DMI and circulating adiponectin, glucose, leptin, MEBAL, and milk yield in FAT and THIN cows at calving. GEE regression was performed for FAT and THIN cows (Table 4.3). In this model, DMI was the dependent variable and adiponectin, glucose, leptin, MEBAL, and milk yield were independent. Parameter estimates for regression models are presented in Table 4.10 for THIN cows at calving and Table 4.11 for FAT cows at calving. Model fitness, regression equations to predict DMI, and R-squared coefficients were presented to (A) for THIN cows at calving and (B) for FAT cows at calving. 
Table 4.10: Regression parameter estimates for prediction of DMI in THIN cows at calving

\begin{tabular}{cccc}
\hline \hline Parameters in the model & $\begin{array}{c}\text { Beta }(\boldsymbol{b}) \\
\text { Coefficient }\end{array}$ & $\mathbf{S E}^{\dagger}$ & $\boldsymbol{P}$ \\
\hline Constant & 8.74 & 1.24 & 0.001 \\
Adiponectin (ADP) & -.043 & 0.010 & 0.001 \\
Milk yield (MY) & .318 & 0.022 & 0.001 \\
MEBAL & .057 & 0.011 & 0.001 \\
Leptin & .031 & 0.086 & 0.72 \\
Glucose (GLU) & -.156 & 0.271 & 0.56 \\
\hline \hline T.
\end{tabular}

Standard error of beta coefficient
Table 4.11: Regression parameter estimates for prediction of DMI in FAT cows at calving

\begin{tabular}{cccc}
\hline \hline Parameters in the model & $\begin{array}{c}\text { Beta }(\boldsymbol{b}) \\
\text { Coefficient }\end{array}$ & SE $^{\dagger}$ & $\boldsymbol{P}$ \\
\hline Constant & 10.71 & 1.32 & 0.001 \\
Adiponectin (ADP) & .047 & 0.028 & 0.097 \\
Milk yield (MY) & .291 & 0.020 & 0.001 \\
MEBAL & .055 & 0.011 & 0.001 \\
Leptin & .014 & 0.145 & 0.92 \\
Glucose (GLU) & -.539 & 0.299 & 0.07 \\
\hline \hline
\end{tabular}

Standard error of beta coefficient 


\subsection{DISCUSSION}

Transition from late pregnancy to early lactation is accompanied by substantial homeostatic and homeorhetic adaptations in energy and nutrient partitioning (Bauman \& Currie, 1980; Beever, 2006). In early lactating cows, increased circulating GH, PRL, NEFA, BOHB, and glucocorticoids (GC) and decreased circulating insulin and leptin, induce metabolic adaptations in liver and insulin dependent tissues that prioritise glucose partitioning to the mammary gland (Jorritsma et al., 2003; Leroy et al., 2008; Leroy et al., 2010). Adiponectin, because of its insulin sensitising actions (Berg et al., 2002; Matsuzawa et al., 2004; Kadowaki \& Yamauchi, 2005), could be another putative regulator of metabolism during the transition from pregnancy to lactation (Mazaki-Tovi et al., 2005; Mitchell et al., 2005). The purposes of this study were to measure circulating and milk adiponectin and to assess the impact of diet and body condition at calving on circulating adiponectin values in dairy cows.

\subsubsection{Measurement of plasma and milk adiponectin}

Circulating and milk adiponectin were measured in this study by using a commercially available human adiponectin kit. There is no validated RIA kit for measuring bovine adiponectin, but the Linco kit has been used for cows (Raddatz et al., 2008), horses (Gordon \& McKeever, 2005; Gordon et al., 2006; Kearns et. al, 2006; Pratt et al., 2005), and dogs (Gayet et al., 2007; Brunson et al., 2007). Bovine adiponectin protein sequence shares about $91 \%$ homology with mouse, and murine adiponectin protein sequence is similar up to $85 \%$ with human, rat, and monkey adiponectin protein sequences (Berg et al., 2002). This high conservation and homology of adiponectin molecule among species and the fact that Linco kit measured both bovine circulating adiponectin and milk adiponectin in this study, plasma adiponectin in another experiment (Raddatz et al., 2008), and circulating adiponectin in other species (Gordon \& McKeever, 2005; Gordon et al., 2006; 
Kearns et. al, 2006; Pratt et al., 2005; Gayet et al., 2007; Brunson et al., 2007), suggest that Linco kit can be used to assess bovine adiponectin.

In this experiment, mean plasma adiponectin varied from $6.5 \pm 0.75 \mathrm{ng} / \mathrm{ml}$ to $11.1 \pm 1.06$ $n g / m l$, and some cows had constantly elevated plasma adiponectin throughout the experiment. Raddatz et al., (2008) reported that plasma adiponectin values varied from $8.3 \pm 1.4 \mathrm{ng} / \mathrm{ml}$ to $16.0 \pm 2.7 \mathrm{ng} / \mathrm{ml}$ in early lactating cows and individual cows had consistently high or low adiponectin levels throughout the sampling period, in agreement with the present study. Circulating adiponectin values were not normally distributed, but were left skewed and highly peaked; this agrees with distributions reported for plasma adiponectin by Cassidy et al. (2009) and Pischon et al. (2005) in humans and Barnea et al. (2006) in mice. Adiponectin concentration in plasma has been reported to be two to three times higher than other hormones (Ahima, 2006); in the present study, mean circulating adiponectin was significantly greater than the mean of insulin, leptin, GH and glucagon. The high autocorrelation of circulating adiponectin values might indicate that plasma adiponectin concentration was tightly genetically controlled and other factors had only minimal impact, but this needs to be further elucidated.

Mean plasma adiponectin was $8.6 \pm 0.33 \mathrm{ng} / \mathrm{ml}$ whereas milk adiponectin was $11.5 \pm 2.60$ $\mathrm{ng} / \mathrm{ml}$. Adiponectin concentrations in human breast milk ranged from 3.9 to $30.4 \mathrm{ng} / \mathrm{ml}$ (Bronsky et al., 2006), and from 4.2 to $87.9 \mathrm{ng} / \mathrm{ml}$ (Martin et al., 2006). Ahima (2006) reported that normal concentrations in human serum ranged from 5 to $30 \mu \mathrm{g} / \mathrm{ml}$. No study directly compared circulating and milk adiponectin. In the present study, milk and plasma adiponectin concentrations were similar, which may imply that milk adiponectin is excreted at concentrations similar to blood, but this hypothesis needs to be investigated further.

In mice and human milk, adiponectin decreases over the course of lactation. Human milk adiponectin concentrations decrease approximately $5 \%-6 \%$ with each month of lactation (Newburg et al., 2010; Savino \& Liguori, 2008). In the present study, milk adiponectin concentrations did not show the same trend, although samples were collected only for the 
first two months of lactation; nevertheless, adiponectin varied with day in milk and the pattern needs to be investigated further. Another finding of this study was that milk adiponectin was not different for cows with high or low milk yield, which also needs to be investigated further.

\subsubsection{The impact of lactational stage on circulating adiponectin}

This study showed that, as in humans (Asai-Sato et al., 2006; Ritterath et al., 2008), the transition from pregnancy to lactation in dairy cows is associated with a reduction in the plasma concentration of adiponectin. The same pattern of decreased AdipoR1 and AdipoR2 mRNA expression in subcutaneous fat tissue during transition from pregnancy (1 week precalving) to lactation (3 weeks postcalving) was reported by Lemor et al. (2009) in high yielding dairy cows. The result of this study is generally in agreement with Komatsu et al. (2007) who reported that adiponectin mRNA expression in adipose tissue was higher in non-lactating cows (dried-off for 3-10 weeks) than cows at peak lactation (8-11 weeks after parturition). The present study generally agrees with Ohtani et al. (2011) who reported that mammary gland adiponectin mRNA expression was lower in early and late lactation compared with non-pregnant or dry cows. Moreover, the present study found that after the $4^{\text {th }}$ week postpartum circulating adiponectin is significantly increased and remains at the same level until the $12^{\text {th }}$ week postpartum. Another study measured circulating adiponectin in cows by using the same RIA kit (Raddatz et al., 2008) reported that circulating adiponectin was significantly increased from $(8.3 \pm 1.4 \mathrm{ng} / \mathrm{ml})$ in the first week to $(16.0 \pm 2.7 \mathrm{ng} / \mathrm{ml})$ at week 4 postpartum and then declined to remain at $12-13 \mathrm{ng} / \mathrm{ml}$ until the $11^{\text {th }}$ week postpartum. This pattern of circulating adiponectin is also in agreement with the pattern found in the present study. Furthermore, this study suggests that after $12^{\text {th }}$ week postpartum there is a significant reduction in circulating adiponectin, whereas plasma adiponectin values do not change during the period from 4 to 12 week postpartum. In the study of Komatsu et al. (2007), adiponectin mRNA expression in adipose tissue from four cows in early lactation (8-11 weeks after parturition) did not differ from that of four cows in late lactation (40-50 weeks after parturition). This result does not directly contradict the 
finding of the present study because Komatsu et al. (2007) measured expression of mRNA in adipose tissue at a much later stage of lactation.

In the present study, stage of lactation affected the majority of circulating metabolic hormones, metabolites, and production traits, but circulating adiponectin showed correlations only with circulating $\mathrm{GH}$ and $\triangle \mathrm{BCS}$, although there was a weak negative association between plasma adiponectin and glucose. The weekly changing pattern of circulating adiponectin can be partially explained by the negative relationship between GH and adiponectin; GH was elevated 2 weeks postpartum and adiponectin reached a nadir at the same point of time. Subsequently, GH decreased steadily until week 12 and then increased slightly, while adiponectin increased until week 4 and then remained stable until week 12 and then decreased. $\triangle \mathrm{BCS}$ was correlated negatively with plasma adiponectin and positively with circulating GH, and cows gained BCS after week 8 postpartum in this experiment, which might be another reason for the decrease in adiponectin between weeks 12 and 15 . This is the first study to demonstrate a negative relationship between GH and adiponectin in dairy cows. However, this relationship needs to be investigated further.

In the present study, milk yield was correlated positively with GH and negatively with adiponectin. This may indicate that $\mathrm{GH}$ is the major regulator of circulating adiponectin postpartum in dairy cows. It is known that genetic selection for milk yield led to higher circulating GH in dairy cows (Veerkamp et al., 2003), which makes the dairy cow the most suitable animal model to study relations between GH and adiponectin. Lam et al. (2004) reported that circulating adiponectin was low in patients who suffered from acromegaly, and that circulating adiponectin increased after GH-lowering therapies. Nilsson et al. (2005) demonstrated that gene expressions of AdipoR1 and AdipoR2 in human adipose tissue are differentially regulated by PRL and GH. Also, PRL and GH reduced adiponectin secretion by human adipose tissue in vitro and in vivo in mice. Rodriguez-Pacheco et al. (2007) investigated if adiponectin plays a central role, similar to leptin, in regulating somatotroph and gonadotroph function. They showed that short-term Adiponectin exposure abolishes both basal and stimulated (by Ghrelin and GnRH) secretion of GH and LH by rat pituitary cells in vitro. According to López-Siguero et al. (2010) GH deficient 
children had higher circulating adiponectin than healthy controls. These studies are in line with the result of the present study as they imply negative correlation between circulating GH and adiponectin.

Other hormonal signals may be associated with changes in circulating adiponectin by week. According to Asai-Sato et al. (2006) circulating adiponectin declined slightly as pregnancy advanced and reached its lowest level during lactation, possibly because PRL affects regulation of maternal metabolism through suppression of adiponectin. Shi et al. (2010) demonstrated that GC decreased adiponectin in rat. TNF- $\alpha$ is another adipokine which is potent negative regulator of systemic insulin sensitivity, which has also been demonstrated to stimulate leptin (Zumbach et al., 1997) but inhibits adiponectin production (Fasshauer et al., 2002; Ruan et al., 2002). It is possibly that elevated GH, PRL, and TNF$\alpha$, and GC could account for diminished circulating adiponectin 2 weeks postpartum, although TNF- $\alpha$, PRL and Glucocorticoids were not measured in the present study.

The role of adiponectin is closely connected to glucose metabolism through enhancing insulin sensitivity and increasing glucose uptake via the GLUT4 transporter (Dridis \& Taouis, 2009). Adiponectin increases insulin sensitivity in isolated primary hepatocytes, resulting in decreased glucose production (Berg et al., 2001). NEFA and glucose are negatively correlated with circulating adiponectin in rodents and humans (Fruebis et al., 2001; Yang et al., 2004; Pischon et al., 2005; Qi et al., 2005; Cassidy et al., 2008) but, in the present study, although circulating adiponectin tended to be negatively correlated with circulating glucose, adiponectin was not correlated with NEFA.

\subsubsection{The impact of diet and BCS at calving on circulating adiponectin}

There was no effect of diet and BCS at calving on circulating adiponectin, even though cows fed on the HS diet had higher insulin but lower BOHB and NEFA than cows fed on the HF diet. Also, diet had no effect on production traits. Circulating insulin, BOHB, and NEFA were not correlated with adiponectin. Yang et al. (2004) observed that rats fed on a 
HF diet showed significant increases in postprandial serum triglycerides, FFA, and insulin but no change in serum glucose and adiponectin. Xu et al. (2007) explored the effect of dietary supplementation of cysteamine on expression of adiponectin and adiponectin receptors in various tissues of rat. They observed no significant change in adiponectin mRNA expression in adipose tissue whereas circulating GH remained unchanged and insulin was higher in the treatment group than in the control. Muhlhausler et al. (2007) reported that maternal plasma glucose and insulin and foetal plasma glucose and insulin were significantly increased in well-fed ewes and their foetuses when compared with control ewes and their foetuses, and this rise was followed by significantly increased circulating adiponectin in both maternal and foetal plasma of well-fed animals. These three studies may suggest that plasma adiponectin concentrations change only when circulating GH or/and glucose are changed, in agreement with the present study. Circulating GH and glucose were not altered by dietary treatments in this experiment, which was possibly the reason diet had no effect on circulating adiponectin.

Body condition score at calving had no effect on metabolic hormones and metabolites, which could be the reason it did not influence circulating adiponectin in this experiment. Plasma GH and glucose concentrations were not different in THIN and FAT cows at calving. Thus, it is possible that circulating adiponectin was unchanged by BCS at calving because this treatment did not influence circulating $\mathrm{GH}$ and glucose which affect circulating adiponectin. However, DMI, milk yield, and $\triangle \mathrm{BCS}$ were higher in FAT cows at calving than THIN cows at calving. $\triangle \mathrm{BCS}$ was correlated positively with $\mathrm{GH}$ and negatively with plasma adiponectin concentrations in this experiment. This means that FAT cows at calving should have had lower circulating adiponectin and higher circulating GH than THIN cows at calving. Unfortunately, this was not the case in the present study. GH was substantially reduced in human obesity (Williams et al., 1984), but in dairy cows genetic improvement has led to animals with higher BCS and circulating GH (Veerkamp et al., 2003). In the present study, BCS at calving was generally high in both condition groups, so the groups consisted of fat to over-fat cows, which might explain the lack of effect on circulating GH. 
A metabolic explanation why in the present study BCS at calving and BCS did not influence adiponectin could be the following. The present study was carried out in pregnant and lactating animals. It is known that pregnancy and lactation require considerable energy uptake and mobilization by the mother (Bauman, 2000). Furthermore, the first half of pregnancy is associated with acute fat mass deposition to ensure proper embryonic development, and the second half of pregnancy and early lactation involve the development of an insulin-resistant state in the mother in order to increase hepatic gluconeogenesis and decrease glucose uptake in maternal muscle and adipose tissue (Bauman \& Currie, 1980; Leroy et al., 2010). This latter adaptation results in maximum supply of maternal glucose to the foetus (in late pregnancy) or mammary gland (in early lactation) and adiponectin, due to its insulin sensitizing effects, will preferably channel nutrients to maternal tissues rather than to foetus or mammary gland. So, it is possible that mammals have developed an alternative mechanism that results in uncoupling of maternal body condition with circulating adiponectin levels. In humans, there is a negative relationship between circulating adiponectin and pre-gestational BMI (Lopez-Bermejo et al., 2004; Retnakaran et al., 2004; Williams et al., 2004), but adiponectin levels throughout pregnancy are not correlated with maternal BMI (Mazaki-Tovi et al., 2005; Jansson et al., 2008). According to Mazaki-Tovi et al. (2005) the disruption of the negative correlation between adiponectin and maternal weight can result from several factors, but dramatic hormonal changes during pregnancy, such as increased levels of oestrogen, prolactin, cortisol, and testosterone that characterize normal human pregnancy, must be the main reason. Moreover, circulating adiponectin showed a strong negative relationship with visceral body fat and visceral body fat is strongly related with BMI in humans (Matsubara et al., 2002; Cnop et al., 2003; Lara-Castro et al., 2006). It is doubtful if BCS is related to visceral body fat of the cow, except for a possible negative correlation in thin cows (Garnsworthy, 2006), and that is possibly another reason why circulating adiponectin was not influenced by BCS at calving. Lemor et al. (2010) showed that mRNAs of both adiponectin receptors were more highly expressed in ovine visceral than in ovine subcutaneous fat tissue, whereas mRNA of adiponectin did not differ. The other adipokine, leptin, measured in the present study was also not affected by BCS at calving, which supports the above suggestion. Also, according to Mousavinasab et al. (2005) only in 
severely obese cases $\left(\mathrm{BMI} \geq 30 \mathrm{~kg} / \mathrm{m}^{2}\right.$ ) with more than $10 \%$ decrease in BMI circulating adiponectin tended to increase in humans, although not statistically significantly.

In the present study, circulating insulin, glucagon, leptin, NEFA, BOHB, and urea were not correlated with circulating adiponectin. Some studies suggest that the relationship between adiponectin and leptin is negative (Matsubara et al., 2002; Huypens, 2007). However, other studies reported positive correlation between leptin and adiponectin (Pardo et al., 2004; Rossi et al., 2005) or no correlation (Park et al., 2004) which was the case in this experiment. In line with this study, Giahi et al. (2008) in overweight diabetic and nondiabetic men and Raddatz et al., (2008) in dairy cows showed that circulating insulin was not correlated with circulating adiponectin. Yamauchi et al. (2001) showed that adiponectin is a putative stimulator of protein synthesis and inhibitor of protein degradation through activation of the insulin signalling pathway, but circulating adiponectin was uncorrelated with plasma urea in this experiment. Also, BCS, milk yield, MEBAL, and LWT were not correlated with circulating adiponectin in this experiment. Raddatz et al. (2008) found no correlation of BCS, insulin, milk yield, DMI, and energy corrected milk yield with adiponectin, in agreement with the present study.

It has been demonstrated that AMPK activation and deactivation in hypothalamic neural cells play a key role in monitoring energy status and regulating food intake (Andersson et al., 2004; Minokoshi et al., 2004; Claret et al., 2007; Lim et al., 2010). Leptin and adiponectin control AMPK with leptin to deactivate (Minokoshi et al., 2008) and adiponectin to activate it (Kubota et al., 2007) in hypothalamic neurons. Receptors of leptin and adiponectin have been found in various hypothalamic regions and presence of leptin and adiponectin have been ascertained in cerebrospinal fluid (Steinberg \& Kemp, 2007). Also, other stimuli, such as insulin and glucose, deactivate AMPK (Claret et al., 2007). Kubota et al., (2007) showed that adiponectin enhances AMPK activity in the murine arcuate hypothalamus through its receptor AdipoR1 to stimulate food intake. In the present study, regression analysis demonstrated that adiponectin is likely to be related with DMI in dairy cows, but this relation is minimal and dependent on BCS at calving. The equations predict a negative relationship between circulating adiponectin and DMI for 
THIN cows at calving, but a positive relationship for FAT cows at calving. In both equations, adiponectin beta coefficients are small compared with beta coefficients of other terms in the models, which suggests a minimal influence of circulating adiponectin on feed intake. Removal of adiponectin from the model reduced $R^{2}$ by $1 \%$ for THIN cows at calving and $0.2 \%$ for FAT cows. Thus, adiponectin has only a marginal positive or negative effect on DMI depending on BCS at calving. However, this finding needs to be investigated further.

AMY (aggregated milk yield at the end of the experiment) and PMY (previous milk yield) positively correlated with milk yield (AMY; $r h o=0.753$, PMY; $r h o=0.473$ ), DMI (AMY; $r h o=0.595$, PMY; rho=0.365), and $\Delta \mathrm{BCS}(\mathrm{AMY} ; r h o=0.434$, PMY; rho=0.281) Also, AMY positively correlated with PMY ( $r h o=0.513)$. Thus, milk yield, DMI, and $\triangle \mathrm{BCS}$ were all correlated positively in the present study. This means that high yielding cows gave more milk, eat more feed, and lost more condition than low yielding cows. Moreover, $\triangle \mathrm{BCS}$ negatively correlated with circulating adiponectin $(r h o=-0.173)$ and leptin $(r h o=-$ 0.261). Therefore, high yielding cows had lower circulating adiponectin and leptin than low yielding cows. In support of this, AMY ( $r h o=-0.117)$ and PMY (rho= -0.256) negatively correlated with circulating adiponectin, whereas PMY negatively correlated with circulating leptin $(r h o=-0.122)$. $\triangle \mathrm{BCS}$ positively correlated with circulating $\mathrm{GH}$ $(r h o=0.253)$ in this study. This means that high yielding cows lost more condition postpartum, and they had higher circulating GH but lower circulating adiponectin and leptin than low yielding cows. In congruence with this, circulating adiponectin ( $r h o=$ $0.144)$ and leptin (rho $=-0.319)$ were negatively correlated with circulating GH. Furthermore, $\triangle \mathrm{BCS}$ positively correlated with milk yield $(r h o=0.388)$, DMI $(r h o=0.382)$, and negatively correlated with circulating insulin $(r h o=-0.158)$. Additionaly, insulin positively correlated with leptin $(r h o=0.356)$ and negatively correlated with circulating $\mathrm{GH}(r h o=-0.287)$ in the present study. To sum up, high yielding cows in this experiment lost more condition postpartum, ate more feed and had higher circulating GH, milk yield and DMI but lower circulating adiponectin, leptin, and insulin than low yielding cows. It is known that selection for a higher yield increases DMI, GH, and BOHB (Veerkamp et al., 2003; Leroy et al., 2010) and this is in agreement with the findings of this study. 
Moreover, high yielding cows lose more condition postpartum than low yielding cows (Roche et al., 2009) and that is in line with the results. Leifers et al. (2005) suggested that leptin expression in adipose tissue is possibly regulated in early lactating cows by the double impact of insulin (positive) and GH (negative), which might be the case in the present study. This is the first study to suggest that due to increased circulating GH and its antagonistic relationship with adiponectin (and leptin), high yielding cows may have lower circulating leptin and adiponectin than low yielding cows. However, this result needs to be elucidated further.

\subsubsection{Conclusions}

To conclude, circulating adiponectin and milk adiponectin were measured in dairy cows in the present study. Milk adiponectin varied by day in milk and it was unaffected by average daily milk yield. Circulating adiponectin was influenced by lactational stage, but was not influenced by diet and BCS at calving. The negative correlation between circulating GH and adiponectin may explain the effect of lactational stage on plasma adiponectin concentrations. Diet and BCS at calving did not influence circulating adiponectin values, mainly because they did not change circulating GH. $\triangle \mathrm{BCS}$ was correlated negatively with leptin and adiponectin and may be a useful tool to study circulating adipokines in periparturient and early lactating cows. Regression analysis showed that adiponectin is likely to be related with DMI in dairy cows, but this relationship is minimal and dependent on BCS at calving. High yielding cows may have lower circulating leptin and adiponectin, and higher circulating GH than low yielding cows. However, these results need to be investigated further. 


\section{Interrelationship of adiponectin with glucose homeostasis, and the effect of circulating adiponectin levels on reproductive performance in dairy cows}

\subsection{INTRODUCTION}

Adiponectin is an adipokine secreted by adipose tissue, which improves the ratio of glucose to FFA and consequently has insulin sensitizing activity (Kadowaki \& Yamauchi, 2005; Ahima, 2006). In the liver, adiponectin decreases glucose output, increases FFA oxidation, and increases influx of NEFA. In muscle tissue, adiponectin increases FFA utilization and stimulates glucose usage (Kadowaki \& Yamauchi, 2005; Garaulet et al., 2007; Shetty et al., 2009).

Lam et al. (2004) reported that circulating adiponectin was low in patients suffered from acromegaly and circulating adiponectin increased after GH-lowering therapies. Nilsson et al. (2005) demonstrated that GH declined adiponectin secretion by human adipose tissue in vitro and in vivo in mice. Rodriguez-Pacheco et al. (2007) presented that short-term adiponectin exposure abolished both basal and stimulated (by Ghrelin and GnRH) secretion of GH by rat pituitary cells in vitro. According to López-Siguero et al. (2010) GH deficient children had higher circulating adiponectin than healthy controls. It is known that genetic selection for milk yield led to higher circulating GH in dairy cows (Veerkamp et $a l ., 2003)$ and that makes dairy cow a suitable animal model to study relations between GH and adiponectin. However, the relationship between GH and adiponectin in dairy cows has not yet been investigated.

Adiponectin and its receptors are present in theca and granulosa cells, oocytes and the corpus luteum (Lord et al., 2005; Ledoux et al., 2006; Ramachandran et al., 2007; Chabrolle et al., 2007a, 2007b). In sows, adiponectin is also present in follicular fluid at a concentration that is estimated to be $80-90 \%$ of the concentration in serum (Ledoux et al., 
2006). Adiponectin may influence steroidogenesis but contradictory results were reported (Ledoux et al., 2006; Lagaly et al., 2008; Chabrolle et al., 2009; Gutman et al., 2009; Pierre et al., 2009; Maillard et al., 2010). It has been hypothesized that adiponectin effects on the ovary are mediated through its insulin-sensitizing traits (Mitchell et al., 2005) and through its effect on the action of IGF-I (Dupont et al., 2008; Michalakis \& Segars, 2010).

The present study utilized the data generated by the previous experiment (data generated by Garnsworthy et al. (2009), in which circulating adiponectin values were measured and added in the working dataset as detailed in Chapter 4). In this dataset, some cows had persistently elevated plasma adiponectin concentrations throughout the experimental period. In line with this, Raddatz et al., (2008) measured circulating adiponectin in twentysix (26) lactating Holstein cows for the first 11 weeks of lactation, and they reported that individual cows had consistently high or low adiponectin levels throughout the sampling period. In the present study, cows were categorized according to high or low circulating adiponectin level. The main hypothesis was that elevated plasma adiponectin concentrations (up to three times) may result in changes in hormonal and metabolic profile, and/or reproductive performance. This hypothesis has not previously been tested in dairy cows. Also, using a multivariate approach, the association of circulating adiponectin with other metabolic hormones and metabolites, and glucose homeostasis were explored in high yielding and low yielding cows. The interrelationships of adiponectin with the metabolic and hormonal components of glucose homeostasis have not been examined previously in dairy cows. 


\subsection{MATERIALS AND METHODS}

\subsubsection{Data}

This study utilized the dataset generated by the study of Garnsworthy et al. (2009). In the current study, circulating adiponectin values were measured and added (Chapter 4). Key points of materials and methods, further statistical analysis, and handling of the data are presented hereinafter.

\subsubsection{Experimental design}

Data generated by the previous experiment (Chapter 4) were used in the present study. In this dataset, diet and BCS at calving had no effect on circulating adiponectin, whereas lactational stage affected plasma adiponectin concentrations. Moreover, some cows had persistently elevated plasma adiponectin concentrations (up to 3 times the average) throughout the experimental period. Scatter plot graphical representation of circulating adiponectin values (Figure 5.1) illustrated that cows with elevated plasma adiponectin concentrations had a tendency to express characteristic peaks of circulating adiponectin for more than two weeks throughout the experimental period. Based on this observation, cows were grouped into a HIGH adiponectin subgroup (6 cows; 57, 94, 146, 219, 330, and 331, average circulating adiponectin; $21.2 \pm 1.32 \mathrm{ng} / \mathrm{ml}$ ) and a NORMAL subgroup (54 cows, average circulating adiponectin; $7.1 \pm 0.23 \mathrm{ng} / \mathrm{ml}$ ). Thus, the main factor studied in this experiment was ADIPONECTIN LEVEL (HIGH versus NORMAL).

The experimental design was a retrospective case-control epidemiological study. This design is highly susceptible to bias error, sources of which are not always obvious. However, there are occasions in which this design is the best choice in practice. This is where the examined trait is rare or takes a long time to develop (Woodward, 1999). 
Multivariate analysis was performed to examine the associations of adiponectin with other metabolic hormones and metabolites, and central glucose homeostasis. This analysis and data arrangement are explained in $\S 5.3 .2$. 


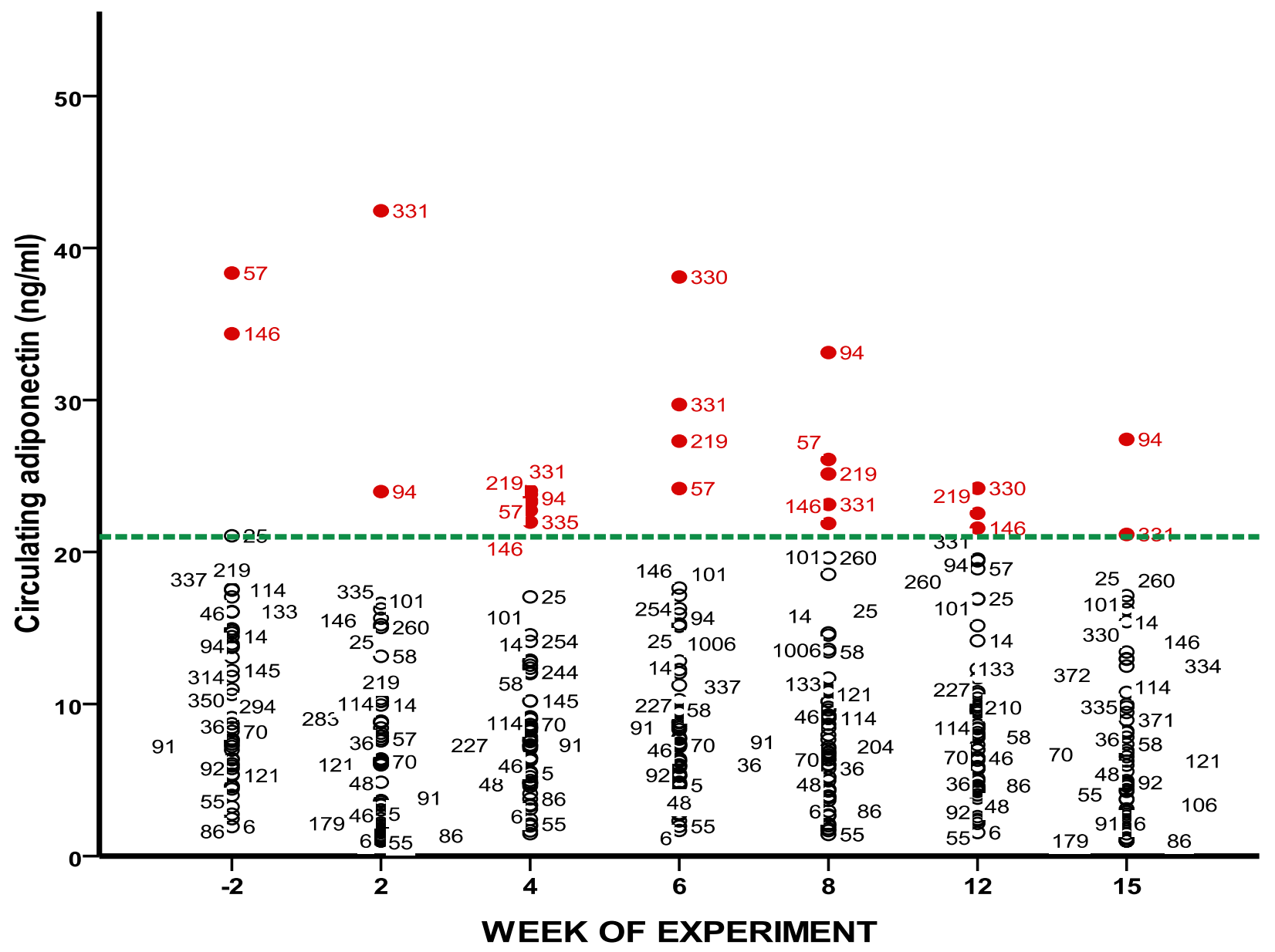

Figure 5.1: Scatter plot graphical representation of circulating adiponectin values $(n=387)$ by week of experiment. Outlier adiponectin values came consistently from 6 cows (id; 57, 94, 146, 219, 330, and 331) which showed the same trend (more than two weeks throughout the experimental period) for higher adiponectin values. 


\subsubsection{Feeding and milking}

These are described in $\S 3.2 .3$.

\subsubsection{Reproductive management}

Cows were artificially inseminated at the first or second oestrus. Insemination was repeated at any subsequent oestrus until the end of the experiment at 120 days post partum. Oestrus was detected using a combination of behavioural observations, pedometer activity monitoring and milk progesterone profiles. Milk progesterone was monitored daily from 4 days before expected oestrus until signs of oestrus were detected; monitoring then returned to twice in a week until 4 days before the next expected oestrus (21 days later). Progesterone profiles were used subsequently to classify oestrous cycles as normal or abnormal (DOV1, DOV2, PCL1 or PCL2), following the definitions of Lamming \& Darwash (1998) (Table A.2 in appendix).

Cows that had not been inseminated, or were not cycling normally, by 90 days post partum were scanned by trans-rectal ultrasound (Aloka SSD-500 scanner equipped with a 5-MHz linear array transducer, Aloka Co., Ltd., Tokyo, Japan) to investigate the existence of cystic body.

\subsubsection{Recording, sampling and analysis}

Milk yield and feed intake were recorded daily throughout the experiment whereas previous milk yield obtained from dairy records. Live weight and BCS (units; 0-5) were recorded weekly whereas $\triangle \mathrm{BCS}$ calculated as BCS at calving minus BCS at 15 week postpartum. Collection and preparation of the samples, and all methods and approaches were used to measure animal productive traits, metabolites, and metabolic hormones are analytically described in the appendices and $\S$ 4.2.2.4. 


\subsection{STATISTICAL ANALYSIS}

\subsubsection{Effect of Adiponectin levels on metabolic and hormonal profiles, and reproductive performance}

Data were analyzed by using PASW ${ }^{\odot} 18$ Edition (SPSS Inc., Chicago, USA). Generalized Estimating Equations (GEE) and Generalized Linear Models (GLM) were used to test specific hypotheses (Lindsey, 1997; McCullagh \& Nelder, 1989; Dobson, 2002; Horton \& Lipsitz, 1999).

Family distribution and link function were selected as detailed in $\S 3.3$. Selection of GLM and GEE models was performed based on AIC and QIC criterion, as detailed in $\S 3.3$.

Parameter estimates of GEE models are presented as marginal means plus/minus standard error of the difference (SED) in tables. Estimated marginal means, SEs and SEDs were obtained by using Least Significant Difference $(L S D)$ adjustment for multiple comparisons in $\mathrm{PASW}^{\odot} 18$ statistical program. $P$ values were calculated by Newton-Raphson Maximum Likelihood $(M L)$ method and effects were considered statistically significant when $P$ value was less than 0.05 . $P$ values equal or less than 0.1 were considered to show a trend.

The GEE and GLM models selected to test the effects of ADIPONECTIN LEVEL on productive traits, reproductive performance, and hormonal and metabolic profile are illustrated by Tables 5.1, 5.2, \& 5.3. The effects of ADIPONECTIN LEVEL and leptin on days to first oestrus (days to oestrus were count data and in such data Poisson distribution function with $\log$ link function fits better) were assessed by fitting GLMs (Tables $5.1 \&$ 5.2). The effect of ADIPONECTIN LEVEL on the probability for cows to be pregnant was examined by fitting a generalized logistic regression model (Table 5.2). Cows non pregnant at the end of the experiment were censored at 120 days post partum and KaplanMeier estimates of the survivor function were compared for cows with HIGH and 
NORMAL adiponectin levels by using Log-rank test (Landau \& Everitt, 2004; Jenkins, 2005; Guo, 2010). The models selected for survival analysis are illustrated in Table 5.4.

Data from milk progesterone profiles were pooled into two groups; cows with NORMAL milk progesterone profile (37 cows) and cows with ABNORMAL milk progesterone profile (23 cows; 5cows with DOV1, 8 cows with DOV2, 1 cow with PCL1, and 9 cows PCL2). In this way, milk progesterone profile was transformed to a binary variable, and causal effects of other continuous and binary variables on it were examined by common logistic regression (Table 5.3). Presence of a follicular cyst at day 90 (CYSTIC BODY) was binary output ( 8 cows were found with follicular cysts at 90 days postpartum; the remaining 52 cows had no cyst) and causal effects were examined by multiple logistic regression models (Table 5.3). The outputs of generalized logistic regression and multiple logistic regression models are presented as probabilities, unstandardized beta $(b)$ coefficients, and odds ratio (exp (b)) with $95 \%$ confidence intervals. Fitness of multiple logistic regression models was assessed as detailed in $\S 3.3$.

Receiver Operating Characteristic (ROC) analysis was conducted to evaluate the performance and the accuracy of logistic regression models, as detailed in $\S 3.3$.

A Shapiro-Wilk test was used to assess whether the distribution of variables was normal. Non-random association between two categorical variables was tested by cross-tabulation and Fischer's exact test. $P$ values equal or less than 0.05 were considered statistically significant whereas $P$ values equal or less than 0.1 were considered to show a trend. 
Table 5.1: Selected models for analysis of the effect of circulating adiponectin levels on metabolites, metabolic hormones, and production traits

\begin{tabular}{|c|c|c|c|c|c|c|c|c|c|c|}
\hline $\begin{array}{c}\text { RESPONSE } \\
\text { VARIABLE (Y) }\end{array}$ & $\begin{array}{l}\text { TYPE OF } \\
\text { MODEL }\end{array}$ & $\begin{array}{l}\text { PREDICTORS } \\
(X)\end{array}$ & $\begin{array}{l}\text { SUBJECT (ID) } \\
\text { VARIABLE }\end{array}$ & $\begin{array}{c}\text { WITHIN- } \\
\text { SUBJECT } \\
\text { VARIABLE }\end{array}$ & $\begin{array}{l}\text { CONTINUOUS } \\
\text { COVARIATES }\end{array}$ & $\begin{array}{c}\text { ERROR } \\
\text { DISTRIBUTION } \\
\text { FUNCTION } \\
\end{array}$ & $\begin{array}{c}\text { LINK } \\
\text { FUNCTION }\end{array}$ & $\begin{array}{l}\text { COVARIANCE } \\
\text { MATRIX }\end{array}$ & $\begin{array}{l}\text { WORKING } \\
\text { CORRELATION } \\
\text { MATRIX }\end{array}$ & STATISTICS \\
\hline $\begin{array}{l}\text {-Insulin } \\
\text { - Glucagon } \\
\text {-Urea } \\
\text {-DMI } \\
\text {-Milk yield } \\
\text {-LW } \\
\text {-MEBAL }\end{array}$ & GEE & $\begin{array}{l}\bullet A D P \\
\text { LEVELS }\end{array}$ & $\begin{array}{l}\text { COW } \\
(60)\end{array}$ & WEEK & PARITY & $\begin{array}{l}\text { Gaussian } \\
\text { (Normal) }\end{array}$ & $\begin{array}{l}\text { Identity } \\
\text { (ID) }\end{array}$ & Robust & Exchangeable & $\begin{array}{l}n=377 ; Q I C=23 \\
n=216 ; Q \mid C=126,067 \\
n=249 ; Q \mid C=167 \\
n=352 ; Q \mid C=4,041 \\
n=352 ; Q \mid C=27,836 \\
n=352 ; Q \mid C=255,866 \\
n=352 ; Q \mid C=85,754\end{array}$ \\
\hline -Adiponectin & GEE & $\begin{array}{l}\text { •ADP } \\
\text { LEVELS }\end{array}$ & $\begin{array}{l}\text { Cow } \\
(60)\end{array}$ & WEEK & PARITY & Gamma & $\log$ & Robust & Exchangeable & $\mathrm{n}=387 ; \mathrm{QIC}=166$ \\
\hline -Leptin & GEE & $\begin{array}{l}\bullet A D P \\
\text { LEVELS }\end{array}$ & $\begin{array}{l}\text { COW } \\
(60)\end{array}$ & WEEK & $\begin{array}{c}\text { PARITY } \\
\text { GH }\end{array}$ & $\begin{array}{l}\text { Gaussian } \\
\text { (Normal) }\end{array}$ & $\begin{array}{l}\text { Identity } \\
\text { (ID) }\end{array}$ & Robust & Exchangeable & $\mathrm{n}=204 ; \mathrm{QIC}=232$ \\
\hline $\begin{array}{l}\bullet \triangle B C S \\
-B C S \text { at } \\
\text { calving }\end{array}$ & GLM & $\begin{array}{l}\text {-ADP } \\
\text { LEVELS }\end{array}$ & - & - & PARITY & $\begin{array}{l}\text { Gaussian } \\
\text { (Normal) }\end{array}$ & $\begin{array}{l}\text { Identity } \\
\text { (ID) }\end{array}$ & Robust & - & $\begin{array}{l}\mathrm{n}=59 ; \mathrm{AIC}=69 ; \quad L R( \\
\left.\chi^{2}=5.9, d f=1, P=0.01\right) \\
\mathrm{n}=60 ; \mathrm{AIC}=35 ; L R( \\
\left.\chi^{2}=4.1, d f=1, P=0.04\right)\end{array}$ \\
\hline •IGF-I & GEE & $\begin{array}{l}\bullet A D P \\
\text { LEVELS }\end{array}$ & $\begin{array}{l}\text { COW } \\
(60)\end{array}$ & WEEK & PARITY & $\begin{array}{l}\text { Gaussian } \\
\text { (Normal) }\end{array}$ & $\begin{array}{l}\text { Identity } \\
\text { (ID) }\end{array}$ & Robust & Exchangeable & $n=197 ; ;$ QIC $=516,092$ \\
\hline -Glucose & GEE & $\begin{array}{l}\bullet A D P \\
\text { LEVELS }\end{array}$ & $\begin{array}{l}\text { COW } \\
(60)\end{array}$ & WEEK & $\begin{array}{l}\text { PARITY } \\
\text { GH } \\
\text { Leptin }\end{array}$ & $\begin{array}{l}\text { Gaussian } \\
\text { (Normal) }\end{array}$ & $\begin{array}{l}\text { Identity } \\
\text { (ID) }\end{array}$ & Robust & Exchangeable & $\mathrm{n}=188 ; \mathrm{QIC}=64$ \\
\hline $\begin{array}{l}\text { - GH } \\
\text {-BOHB } \\
\text {-NEFA }\end{array}$ & GEE & $\begin{array}{l}\bullet A D P \\
\text { LEVELS }\end{array}$ & $\begin{array}{l}\text { COW } \\
(60)\end{array}$ & WEEK & PARITY & $\begin{array}{l}\text { Inverse } \\
\text { Gaussian }\end{array}$ & $\log$ & Robust & Exchangeable & $\begin{array}{l}n=206 ; Q I C=35 \\
n=248 ; Q I C=113 \\
n=249 ; Q I C=333\end{array}$ \\
\hline
\end{tabular}


Table 5.2: Selected models for analysis of reproductive traits

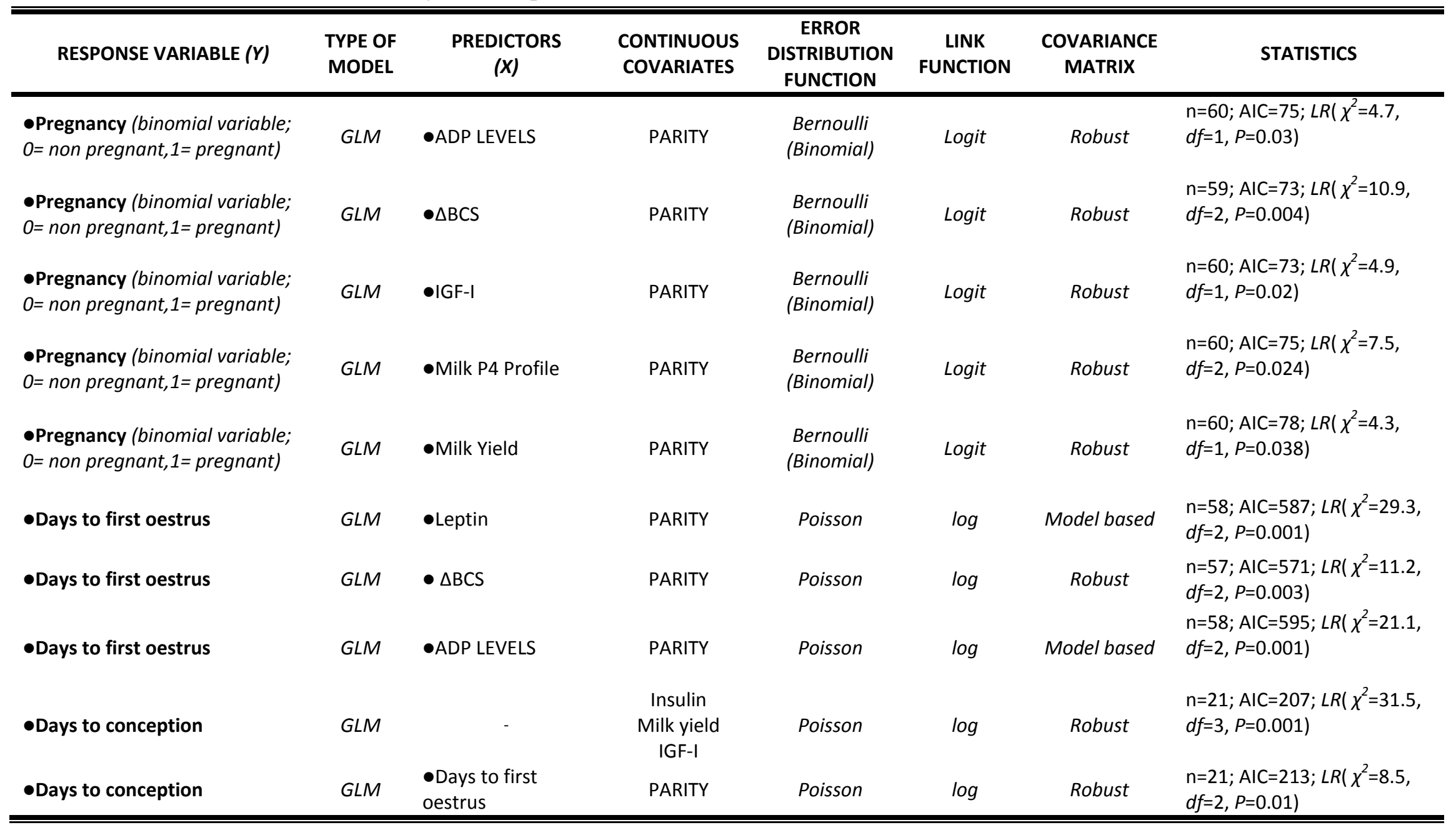


Table 5.3: Selected models for analysis of milk progesterone profile and cystic body

\begin{tabular}{|c|c|c|c|c|c|c|c|}
\hline RESPONSE VARIABLE ( $Y)$ & $\begin{array}{l}\text { TYPE OF } \\
\text { MODEL }\end{array}$ & $\begin{array}{l}\text { PREDICTORS } \\
(X)\end{array}$ & $\begin{array}{l}\text { CONTINUOUS } \\
\text { COVARIATES }\end{array}$ & $\begin{array}{c}\text { ERROR } \\
\text { DISTRIBUTION } \\
\text { FUNCTION } \\
\end{array}$ & $\begin{array}{c}\text { LINK } \\
\text { FUNCTION }\end{array}$ & $\begin{array}{l}\text { COVARIANCE } \\
\text { MATRIX }\end{array}$ & STATISTICS \\
\hline $\begin{array}{l}\text { - MILK P4 PROFILE (binomial } \\
\text { variable; } 0=\text { NORMAL, } 1= \\
\text { ABNORMAL) }\end{array}$ & $G L M$ & - Days to oestrus & PARITY & $\begin{array}{l}\text { Bernoulli } \\
\text { (Binomial) }\end{array}$ & Logit & Robust & $\begin{array}{l}\mathrm{n}=58 ; \mathrm{AIC}=74 ; L R\left(\chi^{2}=9.4,\right. \\
d f=2, P=0.01)\end{array}$ \\
\hline $\begin{array}{l}\text { - MILK P4 PROFILE (binomial } \\
\text { variable; } 0=\text { NORMAL, } 1= \\
\text { ABNORMAL) }\end{array}$ & GLM & - Adiponectin & PARITY & $\begin{array}{l}\text { Bernoulli } \\
\text { (Binomial) }\end{array}$ & Logit & Robust & $\begin{array}{l}\mathrm{n}=60 ; \mathrm{AIC}=74 ; L R\left(\chi^{2}=6,\right. \\
d f=2, P=0.05)\end{array}$ \\
\hline $\begin{array}{l}\text { - MILK P4 PROFILE (binomial } \\
\text { variable; } 0=\text { NORMAL, } 1= \\
\text { ABNORMAL) }\end{array}$ & $\begin{array}{l}\text { Multiple } \\
\text { logistic } \\
\text { regression }\end{array}$ & $\begin{array}{l}\text { - Milk yield } \\
\text {-DMI } \\
\text {-GH }\end{array}$ & PARITY & $\begin{array}{l}\text { Bernoulli } \\
\text { (Binomial) }\end{array}$ & Logit & Robust & $\begin{array}{l}\mathrm{n}=59 ; \mathrm{AIC}=78 ; \operatorname{LR}\left(\chi^{2}=10.6\right. \\
d f=4, P=0.031)\end{array}$ \\
\hline $\begin{array}{l}\text {-CYSTIC BODY (binomial } \\
\text { variable; } 0=\text { no cyst, } \\
1=\text { follicular cyst ) }\end{array}$ & GLM & - Days to oestrus & PARITY & $\begin{array}{l}\text { Bernoulli } \\
\text { (Binomial) }\end{array}$ & Logit & Robust & $\begin{array}{l}\mathrm{n}=58 ; \operatorname{AIC}=45 ; \operatorname{LR}\left(\chi^{2}=7.1\right. \\
d f=2, P=0.03)\end{array}$ \\
\hline $\begin{array}{l}\text {-CYSTIC BODY (binomial } \\
\text { variable; } 0=\text { no cyst, } \\
1=\text { follicular cyst ) }\end{array}$ & $\begin{array}{l}\text { Multiple } \\
\text { logistic } \\
\text { regression }\end{array}$ & $\begin{array}{l}\text {-Insulin } \\
\text { - GH } \\
\text { - Glucose } \\
\text {-NEFA }\end{array}$ & PARITY & $\begin{array}{l}\text { Bernoulli } \\
\text { (Binomial) }\end{array}$ & Logit & Robust & $\begin{array}{l}\mathrm{n}=57 ; \mathrm{AIC}=32 ; \mathrm{LR}\left(\chi^{2}=25.3\right. \\
d f=5, P=0.001)\end{array}$ \\
\hline
\end{tabular}

Table 5.4: Selected models for survival analysis (Kaplan- Meier models) of interval from calving to conception

\begin{tabular}{|c|c|c|c|c|c|c|c|}
\hline $\begin{array}{l}\text { TIME } \\
\text { VARIABLE } \\
\text { (Survival time) } \\
\end{array}$ & $\begin{array}{l}\text { TYPE OF } \\
\text { MODEL }\end{array}$ & $\begin{array}{c}\text { STATUS } \\
\text { VARIABLE (Event } \\
\text { variable) }\end{array}$ & $\begin{array}{c}\text { FACTORS } \\
\text { (Group variables) }\end{array}$ & $\begin{array}{c}\text { SURVIVAL } \\
\text { FUNCTIONS } \\
\text { COMPARISON TEST }\end{array}$ & OBSERVATIONS & $\begin{array}{c}\text { RIGHT } \\
\text { CENCORED } \\
\text { OBSERVATIONS }\end{array}$ & $\begin{array}{l}\text { UNCENCORED } \\
\text { OBSERVATIONS }\end{array}$ \\
\hline $\begin{array}{l}\text { Days post } \\
\text { partum (0- } 120 \\
\text { days post } \\
\text { partum) }\end{array}$ & $\begin{array}{l}\text { Kaplan- } \\
\text { Meier }\end{array}$ & $\begin{array}{c}\text { Pregnancy } \\
\text { (binomial variable; } \\
0=\text { non pregnant } \\
\text { 1=event= pregnant) }\end{array}$ & $\bullet A D P$ LEVELS & $\begin{array}{c}\text { Log-Rank } \\
\text { (Mantel-Cox) }\end{array}$ & 60 & 39 & 21 \\
\hline
\end{tabular}




\subsubsection{Exploration of adiponectin interrelationships with glucose, BCS, metabolic hormones, and metabolites: a multivariate approach}

Data were analyzed by using AMOS $^{\complement}$ (AMOS Development Corporation, Spring House, PA, USA). $\operatorname{AMOS}^{\odot}$ incorporates structural equation modelling (SEM) and path diagrams analysis (PDA). AMOS $^{\odot}$ is a relatively new graphical SEM analysis tool that can fit multiple models in a single analysis by constraining parameters within the models (Cunningham \& Wang, 2005; Arbuckle, 2007). SEM and PDA enable researchers to answer a set of interrelated research questions in a single, systematic, and comprehensive analysis by modelling the relationships among multiple independent and dependent variables simultaneously (Gefen et al., 2000; Ahn, 2002; Kline, 2005). The SEM approach is asymptotically the same as classical regression when its assumptions are satisfied. Regression works by minimizing variance but SEM is based on methods of maximization of likelihood (Gefen et al., 2000). The main assumption of SEM is achievement of multivariate normality, but deviations from multivariate normality may or may not affect the result of analysis (Kline, 2005; Cunningham \& Wang, 2005; Arbuckle, 2007).

The data used for this analysis were averaged by cow for each measured trait, metabolite, and metabolic hormone (i.e. one value per cow for adiponectin, leptin, etc) across the whole experimental period. Using this "main data set", a basic model was constructed for circulating glucose and its interrelationships with adiponectin, insulin, leptin, glucagon, $\mathrm{GH}, \mathrm{BOHB}, \mathrm{NEFA}$, and BCS. The fitness of this basic model was ascertained by using fit indices and chi-square statistics and the data were imputed by using Bayesian imputation (MCMC algorithm, for 10,000 observations assuming 0.1 autocorrelation) (Jackman, 2004; Dunson et al., 2005; Carter, 2006; Arbuckle, 2007). In this way, ten complete data sets plus the original were generated. The data sets were appended to the main data set and averaged by cow. Finally, a complete data set was created, containing one value for each measured trait, metabolite, and metabolic hormone for each individual cow. In this complete data set, the validity of the basic model was tested again using fit indices and chisquare statistics and a 1,000 samples Bollen-Stine bootstrap for nonparametric data. For 
non-normal data, Bollen-Stine bootstrap can provide the correct $\mathrm{P}$ values for the chi-square statistic to assess overall model fit. Bollen-Stine bootstrap uses the same chi-square hypothesis structure, at the conventional significance level of 0.05 , to test the fitness of the model. If the $P$ value is larger than 0.05 the model is accepted, and thus conclude that the model fits the data well, whereas the model is rejected if $P$ value is smaller than 0.05 (Arbuckle, 2007). The basic model showed excellent fitness to the data, so it was used as the source model for constructing further models.

Cows were classified according to milk yield in the previous lactation (PMY) with a cutoff point between high and low yielding cows of 10,000 kg/cow/lactation. PMY was modelled as a factor with two levels; LOW yielders (30 cows, 8,044 $\pm 395 \mathrm{~kg} / \mathrm{lactation}$ ) and HIGH yielders (30 cows, 12,726 $\pm 366 \mathrm{~kg} /$ lactation). DMI, milk yield, $\triangle \mathrm{BCS}$, and BCS at calving were assessed by multivariate analysis of variance (MANOVA) for statistically significant differences between PMY groups in the present lactation. Wilks' lambda $(\lambda)$ test was used to examine the overall significance of the model. When the overall model was significant $(P<0.05)$, then simultaneous effects of the independent variables on the dependent variable existed. Box's test was used to test the equality of covariance between the groups with insignificant $P$ values $(P \geq 0.05)$ to denote that covariance matrices of the dependent variable were equal across groups. Levene's test examined whether or not the variance between independent variable groups was equal. Non-significant $P$ values $(P \geq 0.05)$ of Levene's test denoted equal variance between groups. Power was the probability of correctly accepting or rejecting hypotheses based on the results of the test (Field, 2005; PASW ${ }^{\odot} 18$ Users guide, 2009). Differences in parity between PMY groups were assessed by Student's t-test (two-sided). This analysis was performed to confirm LOW PMY cows and HIGH PMY cows were low yielding and high yielding cows, respectively, in the present lactation. Because the animals retrospectively allocated to PMY groups, a Fisher's exact test performed to assess if PMY blocking of animals was biased by the original dietary treatments.

Glucose interrelationships with adiponectin, metabolites, and metabolic hormones in cows with HIGH and LOW previous milk yield were examined by applying and modifying the 
basic model in the "main data set", so that the maximum fitness to be achieved, and under the condition that previous milk yield was HIGH or LOW. The validity and the fit of the models derived from the basic model were tested by model fit indices (Table 5.5) and comparison of the original correlation matrices with the model-based correlation matrices (Schreiber et al., 2006; Arbuckle, 2007). Also, models were run by Bayesian Estimation method and the Deviance Information Criterion (DIC) was used to determine the fit of the models to the data.

Model parameter estimates (mean $\pm \mathrm{SE}$ ) are illustrated in Table B.1 in appendix.

Table 5.5: Model fit criteria and acceptable fit interpretation

\begin{tabular}{|c|c|c|}
\hline Model Fit Criterion & Acceptable level & Interpretation \\
\hline $\begin{array}{c}- \text { Chi-square statistics } \\
\left(\chi^{2}\right)\end{array}$ & $P>0.05$ indicates good fit & $\begin{array}{l}\text { Compares obtained chi-square } \\
\text { value with table value } \\
\text { for given degrees of freedom }\end{array}$ \\
\hline $\begin{array}{l}\bullet \text { Bollen-Stine } \\
\text { nonparametric bootstrap }\end{array}$ & $P>0.05$ indicates good fit & $\begin{array}{l}\text { Provide the correct } P \text { value for } \\
\text { chi-square statistic when data } \\
\text { are non-normal. }\end{array}$ \\
\hline $\begin{array}{l}\bullet \text { Comparative fitness } \\
\text { index }(\boldsymbol{C F I})\end{array}$ & 0 (no fit) to 1 (perfect fit) & $\begin{array}{l}\text { Value close to } 0.95 \text { reflects a } \\
\text { good model fit }\end{array}$ \\
\hline Normed fit index (NFI) & 0 (no fit) to 1 ( perfect fit) & $\begin{array}{l}\text { Value close to } 0.95 \text { reflects a } \\
\text { good model fit }\end{array}$ \\
\hline $\begin{array}{l}- \text { Root-mean-square } \\
\text { error of approximation } \\
\text { (RMSEA) }\end{array}$ & $\begin{array}{l}<0.05(\text { good fit model }) \\
>1.0(\text { not acceptable })\end{array}$ & $\begin{array}{l}\text { Value less than } 0.05 \text { or } 0.08 \\
\text { indicates a good model fit } \\
\text { Value more than } 1.0 \text { indicates } \\
\text { the model is not } \\
\text { acceptable }\end{array}$ \\
\hline $\begin{array}{l}\text { - Akaike's information } \\
\text { criterion(AIC) }\end{array}$ & $\begin{array}{c}\text { The smaller value is the } \\
\text { better }\end{array}$ & $\begin{array}{l}\text { Compares values in alternative } \\
\text { models }\end{array}$ \\
\hline $\begin{array}{l}\text { - Deviance Information } \\
\text { Criterion (DIC) }\end{array}$ & $\begin{array}{l}\text { Posterior predictive } P \\
\text { value should be near } 0.5 \\
\text { for a correct model, with } \\
\text { values toward the extremes } \\
\text { of } 0 \text { or } 1 \text { indicating that a } \\
\text { model is not plausible }\end{array}$ & $\begin{array}{l}\text { DIC is for comparing the fit of } \\
\text { alternative models, with smaller } \\
\text { values being better. DIC cannot } \\
\text { be used to evaluate a single } \\
\text { model in absolute terms. }\end{array}$ \\
\hline
\end{tabular}

Source: Arbuckle, 2007; Kline, 2005; Bollen \& Curran, 2006 


\subsection{RESULTS}

\subsubsection{The effect of circulating adiponectin levels on metabolic and hormonal profile, production traits, and reproduction}

\subsubsection{Reproductive performance}

Interval from calving to conception between cows with HIGH adiponectin levels and cows with NORMAL adiponectin levels were not statistically significant different (Figure 5.2). Cows with NORMAL milk progesterone profiles were more likely to become pregnant ( $48 \%$ versus $\left.10 \%, \mathrm{AUC}_{R O C}=0.73 \pm 0.06\right)$ during the experimental period than cows with ABNORMAL milk progesterone profiles $(P<0.05)$ (Table 5.6). Cows with HIGH adiponectin levels had a higher probability to be pregnant (83\% versus $30 \%$, $\left.\mathrm{AUC}_{R O C}=0.67 \pm 0.07\right)$ during the experimental period than cows with NORMAL adiponectin levels $(P<0.05)$ (Table 5.7). Cows with HIGH adiponectin levels had lower days to first oestrus (43.1 versus 56) than cows with NORMAL adiponectin levels $(P<0.05)$ (Table 5.7). There was a tendency for non-random association between cows with HIGH adiponectin levels and cows with NORMAL milk progesterone profile $(P<0.1)$ because no cow with HIGH adiponectin had ABNORMAL profiles (Table 5.8). There was no association between adiponectin level and presence of follicular cysts at day 90 of lactation (Table 5.9).

Logistic regression analysis showed a significant association between change in BCS $(\triangle \mathrm{BCS})$ and the probability for a cow to be pregnant. Cows losing 0.25 units of BCS or less were more likely to become pregnant ( $56 \%$ versus $30 \%, \mathrm{AUC}_{R O C}=0.72 \pm 0.06$ ) than cows losing 0.70 units of BCS or more (Figure 5.3). The association between circulating IGF-I and probability to be pregnant was significant. Cows with average circulating IGF-I equal or greater than $155 \mathrm{ng} / \mathrm{ml}$ were more likely to be pregnant (46\% versus $33 \%$, $\left.\mathrm{AUC}_{R O C}=0.70 \pm 0.07\right)$ than cows with average circulating IGF-I equal or less than $120 \mathrm{ng} / \mathrm{ml}$ 
throughout the experimental period (Figure 5.4). Days to first oestrus were significantly associated with the probability of cows to express ABNORMAL milk progesterone profiles. Cows with average 43.1 days to first oestrus were less likely ( $25 \%$ versus $36 \%$, $\left.\mathrm{AUC}_{R O C}=0.72 \pm 0.07\right)$ to express $\mathrm{ABNORMAL}$ milk progesterone profiles than cows with average 56 days to first oestrus (Figure 5.5), whereas days to first oestrus were significantly associated with the probability for cows to express cystic body at day 90 postpartum (Figure 5.6). Moreover, $\triangle \mathrm{BCS}$ significantly associated with days to first oestrus (Figure 5.7) and days to first oestrus positively associated with days to conception (Figure 5.8). Circulating adiponectin was significantly associated with the probability for cows to express ABNORMAL milk progesterone profiles (Figure 5.9), whereas circulating leptin was significantly associated with the days to first oestrus (Figure 5.10). Milk yield was significantly associated with the probability of cows to be pregnant (Figure 5.11).

Multiple logistic regression analysis examined the effects of milk yield, DMI, GH, and parity on milk progesterone profile. Each exponentiated coefficient in this model is the change in odds for a unit increase in the corresponding predictor variable holding other variables at certain value. Odds ratio $(\exp (b))$ for milk yield was $1.21(P<0.05)$ (Table 5.10). So holding GH, DMI and parity at a fixed value, an increase of milk yield by $1 \mathrm{~kg} / \mathrm{d}$ will increase the odds for cows to express ABNORMAL milk progesterone profile by $21 \%$. GH and DMI tended to influence milk progesterone profile $(P<0.1)$. GH odds ratio was $1.31(P=0.07)$; DMI odds ratio was $0.68(P=0.07)$ (Table 5.10). The odds ratio for $\mathrm{GH}$ and DMI say that, holding the other predictors of the model at a fixed value, a one-unit increase in GH was expected to result in a $31 \%$ increase, and a one-unit increase in DMI a $32 \%$ decrease, in the odds of cows to express ABNORMAL milk progesterone profiles. Parity also tended to influence milk progesterone profile $(P<0.1)$. The odds ratio for parity says that, holding the other predictors of the model at a fixed value, an increase of parity by one year resulting in an $86 \%$ rise in the odds of cows to express ABNORMAL milk progesterone profiles.

Multiple logistic regression analysis examined the effects of insulin, glucose, GH, NEFA, and parity on presence of follicular cysts at 90 days postpartum (Table 5.11). Odds ratios 
for insulin and glucose were close to zero and that means strong negative effects on follicular cyst formation $(P<0.05)$. Odds ratios for GH and NEFA were 2.1 and 201.6 respectively, indicating high positive effects of GH and NEFA on follicular cyst formation $(P<0.05)$. Parity had no effect on follicular cyst formation.

Multiple poisson regression analysis examined the effects of insulin, milk yield, and IGF-I on days to conception (Table 5.12). Insulin, milk yield, and IGF-I beta coefficients were $0.98,0.014$, and -0.002 , respectively $(P<0.05)$. Beta coefficient of insulin is bigger than the beta coefficients of milk yield and IGF-I and that means the insulin effect on days to conception is bigger than the effect of milk yield and IGF-I. Insulin concentration greater than $0.7 \mathrm{ng} / \mathrm{ml}$ resulted in calving to conception interval greater than 100 days, whereas animals with insulin concentration within the range 0.2-0.3 $\mathrm{ng} / \mathrm{ml}$ had a calving to conception interval of less than 70 days (Table 5.13).

\subsubsection{Metabolic profile, hormonal profile, and productive traits}

Cows with HIGH adiponectin levels had higher circulating adiponectin and IGF-I, but lower circulating GH and glucose than cows with NORMAL adiponectin levels $(P<0.05)$ (Table 5.14). Cows with HIGH adiponectin levels showed a tendency for higher plasma concentrations of leptin, but lower circulating BOHB than cows with NORMAL adiponectin levels $(P<0.1)$. Adiponectin levels had no effect on plasma concentrations of insulin, glucagon, NEFA, and blood urea (Table 5.14).

Cows with HIGH adiponectin levels had lower DMI, milk yield, BCS at calving, and $\triangle B C S$ than cows with NORMAL adiponectin levels $(P<0.05)($ Table 5.15). Adiponectin levels had no effect on LWT and MEBAL (Table 5.15). 


\subsubsection{Adiponectin interrelationships with glucose, BCS, metabolic hormones, and metabolites}

There was no association between PMY and diet, and thus the retrospective classification of cows as PMY LOW and PMY HIGH was not biased by the original dietary treatments (Fisher's exact test, $\mathrm{n}=60, \mathrm{P}=0.325$, two-sided).

Multivariate analysis revealed that glucose balance in dairy cows was regulated by the negative influences of circulating adiponectin $(-0.26, P=0.014)$, BOHB $(-0.36, P=0.001)$, and glucagon $(-0.23, P=0.036)$ on circulating glucose, and the positive influence of circulating insulin $(0.24, P=0.036)$ on circulating glucose (Figure 5.12). GH, NEFA, leptin, and BCS had no direct effect on circulating glucose, but GH was negatively correlated with adiponectin $(-0.24, P=0.042)$ and leptin $(-0.35, P=0.001)$ whereas leptin was positively correlated with insulin $(0.34, P=0.012)$ (Figure 5.12).

In low yielding cows, glucose balance was regulated by the negative influences of circulating adiponectin $(-0.31, P=0.041)$ and glucagon $(-0.31, P=0.043)$ on circulating glucose, and the positive influence of circulating insulin $(0.44, P=0.008)$ on circulating glucose (Figure 5.13). GH, NEFA, leptin, and BCS had no direct effect on circulating glucose, but leptin was positively correlated with insulin $(0.42, P=0.022)$.

In high yielding cows, glucose balance was regulated by the negative influences of BCS ($0.51, P=0.01)$ and $\mathrm{BOHB}(-0.48, P=0.001)$, and the positive influence of circulating insulin $(0.45, P=0.035)$ on circulating glucose (Figure 5.14). GH, NEFA, leptin, and glucagon had no direct effect on circulating glucose. However, a multitude of interrelations between metabolites and hormones was revealed. Glucagon was positively correlated with leptin (0.48, $P=0.004)$, but negatively correlated with $\operatorname{BCS}(-0.28, P=0.049)$. Adiponectin was positively correlated with $\mathrm{BCS}(0.34, P=0.031)$, but negatively correlated with GH $(-0.34$, $P=0.031)$. GH was negatively correlated with $\mathrm{BOHB}(-0.31, P=0.05)$, BCS $(-0.40$, $P=0.027)$, and leptin $(-0.44, P=0.01)$. Leptin was positively correlated with NEFA $(0.29$, 
$P=0.003)$. Insulin was positively correlated with NEFA $(0.45, P=0.02)$, BCS $(0.58$, $P=0.027)$, and leptin $(0.39, P=0.02)$.

One-way MANOVA revealed a significant multivariate main effect for PMY (Wilks' $\lambda=$ $0.787, \mathrm{~F}=3.66, P=0.01, d f=54$, Box's test $P=0.112$, Power $=0.850)$. Given the significance of the overall MANOVA model, the univariate main effects were examined. High PMY cows had higher milk yield $(47.1 \pm 1.27 \mathrm{~kg} / \mathrm{d}$ versus $40.3 \pm 1.25 \mathrm{~kg} / \mathrm{d}, \mathrm{F}=14.4, \mathrm{df}=57$, $P=0.001$, Levene's test $P=0.937$, Power=0.962), DMI (22.5 $\pm 0.47 \mathrm{~kg} / \mathrm{d}$ versus $20.5 \pm 0.46$ $\mathrm{kg} / \mathrm{d}, \mathrm{F}=8.7, d f=57, P=0.006$, Levene's test $P=0.466$, Power=0.827), and $\mathrm{BCS}$ at calving (3.52 \pm 0.050 units versus $3.35 \pm 0.060$ units, $\mathrm{F}=4.6, d f=57, P=0.035$, Levene's test $P=0.005$, Power $=0.563)$, and a trend for higher $\triangle \mathrm{BCS}(0.76 \pm 0.082$ units versus $0.56 \pm 0.082$ units, $\mathrm{F}=2.54, d f=57, P=0.1$, Levene's test $P=0.662$, Power=0.347) than low PMY cows in the present lactation.

Parity was not statistical different for high PMY cows compared to low PMY cows (2.77 \pm 0.190 lactations versus $2.40 \pm 0.190$ lactations, $t=1.39, d f=58, P=0.17$ ). 


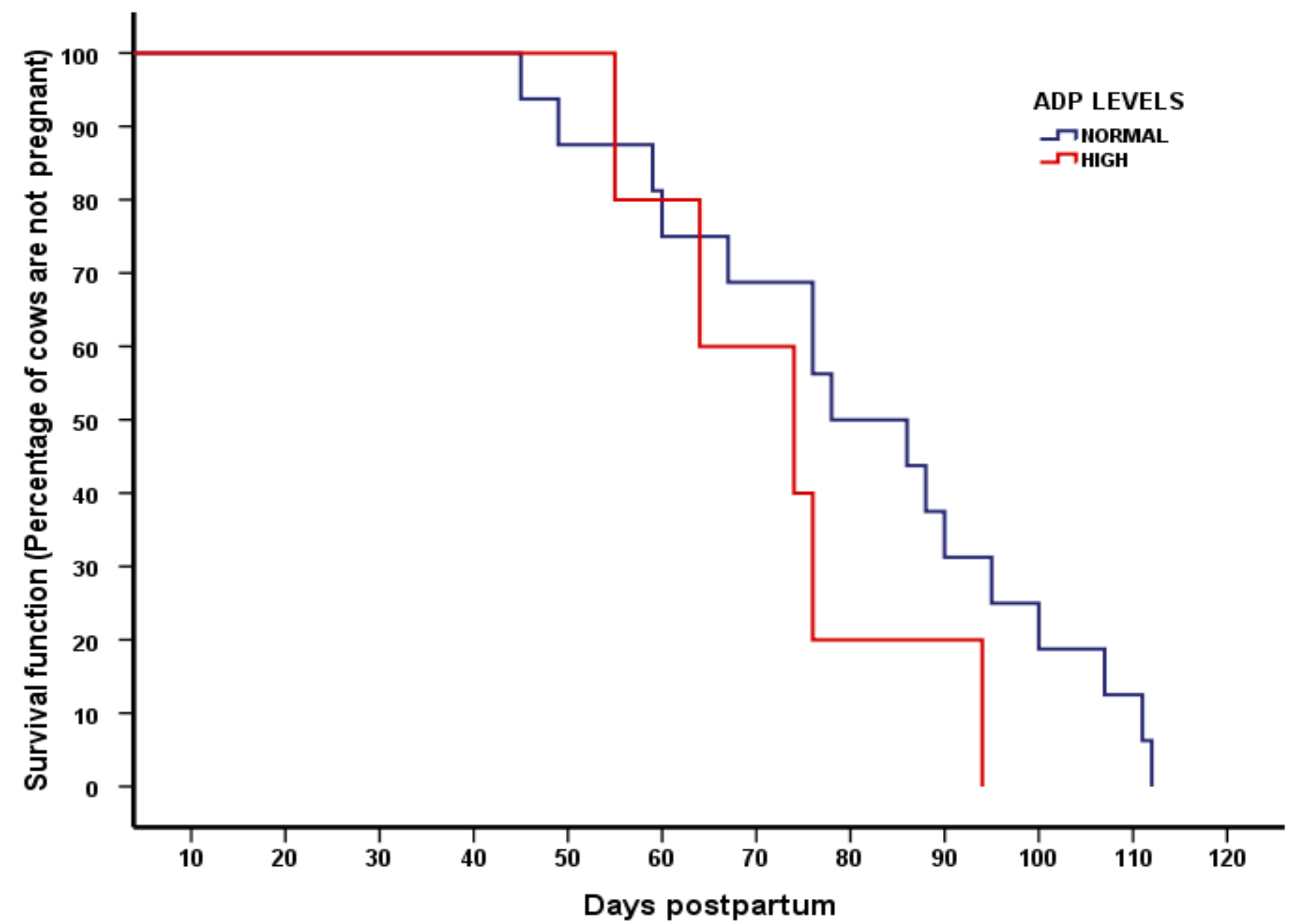

Figure 5.2: Calving-to-conception survival analysis curves for cows with HIGH and NORMAL adiponectin (ADP) levels. There was no difference in the survival curves for cows with HIGH and NORMAL adiponectin levels $(n=60$, Log-rank, $\mathrm{P}=0.18)$. Vertical drop in the survival curves indicates an event (a cow became pregnant). Interval from calving to conception between cows with HIGH (74 \pm 10.9 days) and NORMAL ( $78 \pm 10.0$ days) adiponectin levels were not significantly different. 
Table 5.6: Effect of milk progesterone profile on probability of cows to be pregnant

\begin{tabular}{|c|c|c|c|c|}
\hline \multirow{2}{*}{$\begin{array}{c}\text { Treatment: } \\
\text { Parameters: }\end{array}$} & \multicolumn{2}{|c|}{ MILK PROGESTERONE PROFILE } & \multirow[b]{2}{*}{$n$} & \multirow[b]{2}{*}{$\boldsymbol{P}$} \\
\hline & NORMAL $^{\dagger}$ & ABNORMAL $^{\dagger}$ & & \\
\hline Probability for cows to be pregnant & $0.48(0.32,0.65)$ & $0.01(0.00,0.27)$ & 60 & 0.001 \\
\hline
\end{tabular}

Table 5.7: Effect of circulating adiponectin levels (ADP LEVELS) on probability of cows to be pregnant

\begin{tabular}{|c|c|c|c|c|}
\hline \multirow{2}{*}{$\begin{array}{c}\text { Treatment: } \\
\text { Parameters: }\end{array}$} & \multicolumn{2}{|c|}{ ADP LEVELS } & \multirow[b]{2}{*}{$n$} & \multirow[b]{2}{*}{$\boldsymbol{P}$} \\
\hline & NORMAL $^{\dagger}$ & HIGH $^{\dagger}$ & & \\
\hline $\begin{array}{l}\text { Days to first oestrus } \\
(\text { days postpartum })\end{array}$ & $56.0(51.6,60.4)$ & $43.1(36.8,49.3)$ & 56 & 0.001 \\
\hline Probability for cows to be pregnant & $0.30(0.17,0.42)$ & $0.83(0.53,0.99)$ & 60 & 0.031 \\
\hline
\end{tabular}

† Columns are means with $95 \%$ Confidence Interval (CI). 
Table 5.8: Crosstabulation of circulating adiponectin levels (ADP LEVELS) with milk progesterone profile. Association between ADP LEVELS and milk progesterone profile was tested by performing Fisher's exact test ( $\mathrm{n}=60, P=0.073$, two-sided). There was a tendency for non-random association between cows expressing HIGH circulating adiponectin levels and NORMAL milk progesterone profile, and between cows expressing NORMAL adiponectin levels and ABNORMAL milk progesterone profile.

\section{ADP LEVELS}

\begin{tabular}{cccc}
\hline Milk progesterone profile & NORMAL & HIGH & Total \\
NORMAL & 31 & 6 & 37 \\
ABNORMAL & 23 & 0 & 23 \\
Total & 54 & 6 & 60 \\
\hline \hline
\end{tabular}

Table 5.9: Crosstabulation of circulating adiponectin levels (ADP LEVELS) with follicular cysts found at day 90 postpartum (CYSTIC BODY). Association between ADP LEVELS and CYSTIC BODY was tested by performing Fisher's exact test $(\mathrm{n}=60, P=0.585$, two-sided $)$. There was no association between adiponectin level and cystic body.

ADP LEVELS

\begin{tabular}{cccc}
\hline CYSTIC BODY & NORMAL & HIGH & Total \\
NO & 46 & 6 & 52 \\
YES & 8 & 0 & 8 \\
Total & 54 & 0 & 60 \\
\hline \hline
\end{tabular}




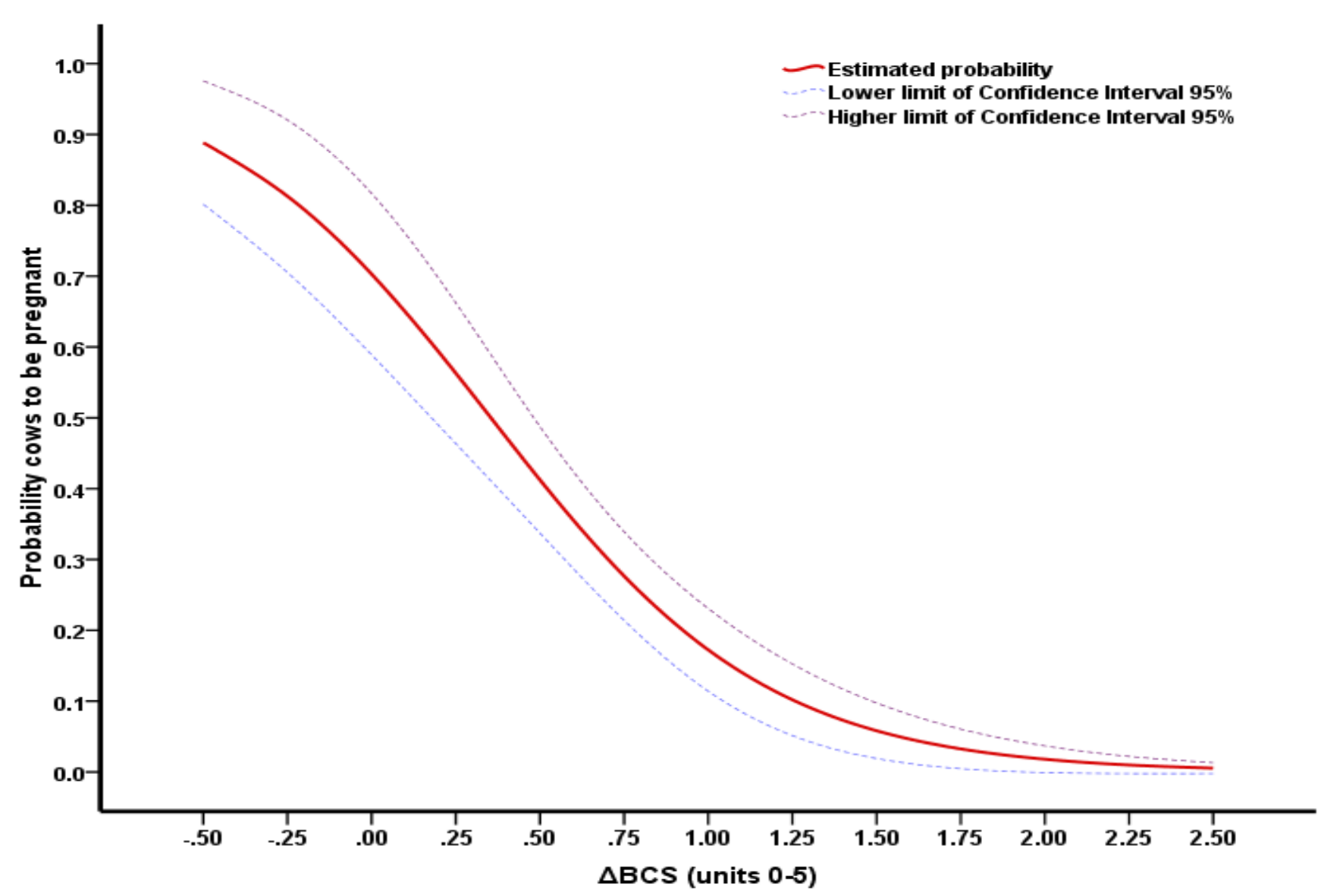

Figure 5.3: Effect of postpartum change in body condition score $(\Delta \mathbf{B C S})$ on probability of cows to be pregnant $(\mathrm{n}=59, P=0.001$, exp $(b)$ $=0.09$, CI for $\exp (b) ;[0.02,0.37]$, beta coefficient $(b)=-2.43, \mathrm{AUC}_{R O C}=0.72 \pm 0.06$, GLM with Bernoulli error distribution, logit link function, and robust standard error estimation). In this model, dependent variable was pregnancy $(0=$ non pregnant, $1=$ pregnant $)$ whereas $\triangle \mathrm{BCS}$ and parity were added as continuous covariates. Red solid line represents the estimated probability whereas blue and purple dashed lines show the upper and lower $95 \%$ Confidence Interval (CI). $\triangle \mathrm{BCS}$ values with negative sign indicate gain of BCS whereas positive $\triangle \mathrm{BCS}$ values denote loss of BCS. 


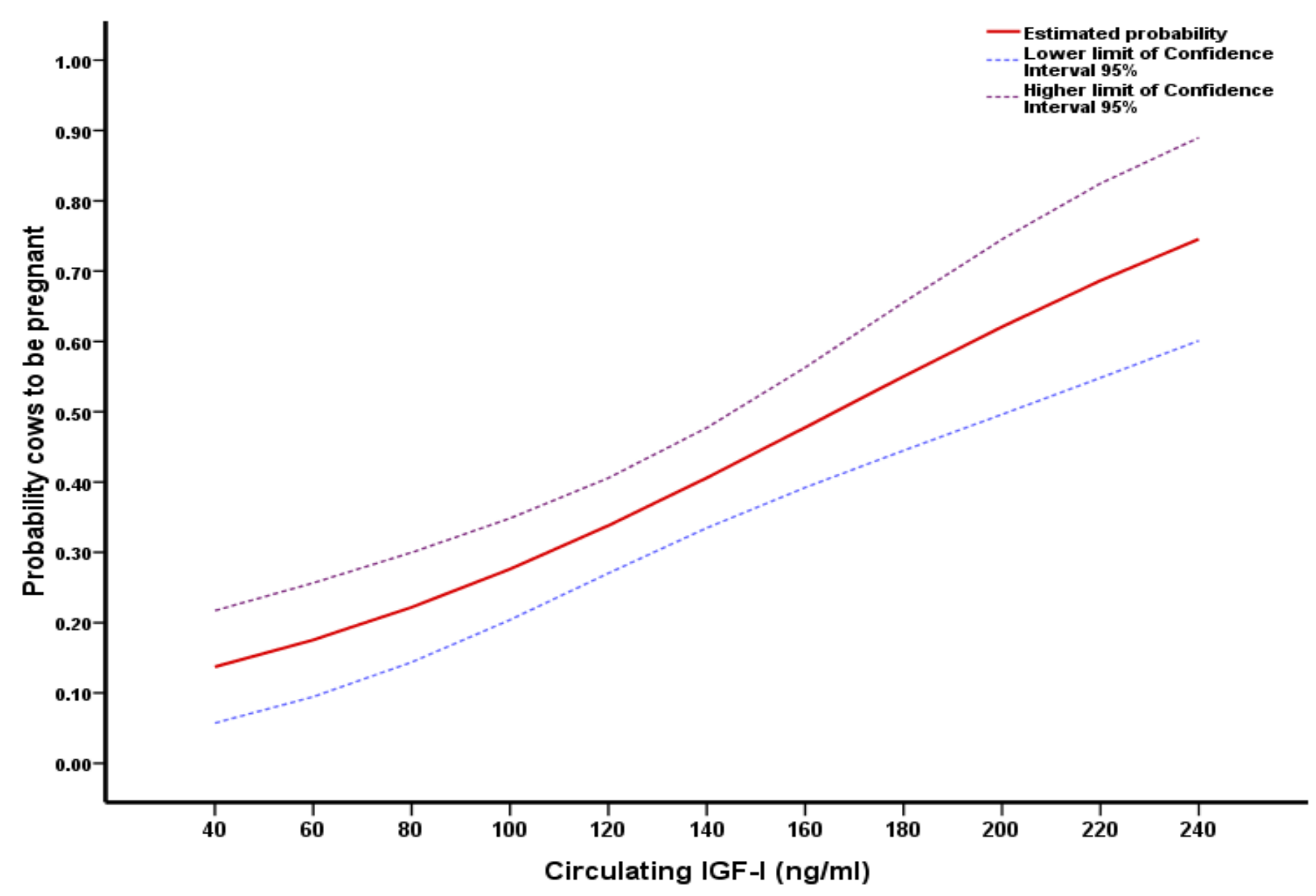

Figure 5.4: Effect of circulating IGF-I on probability of cows to be pregnant $(\mathrm{n}=60, P=0.027, \exp (b)=1.01, \mathrm{CI}$ for $\exp (b)$; [1.002, 1.028$]$, beta coefficient $(b)=0.015, \mathrm{AUC}_{R O C}=0.70 \pm 0.07$, GLM with Bernoulli error distribution, logit link function, and robust standard error estimation). In this model, dependent variable was pregnancy $(0=$ non pregnant, $1=$ pregnant $)$ whereas circulating IGF-I and parity were added as continuous covariates. Red solid line represents the estimated probability whereas blue and purple dashed lines show the upper and lower 95 $\%$ Confidence Interval (CI). 


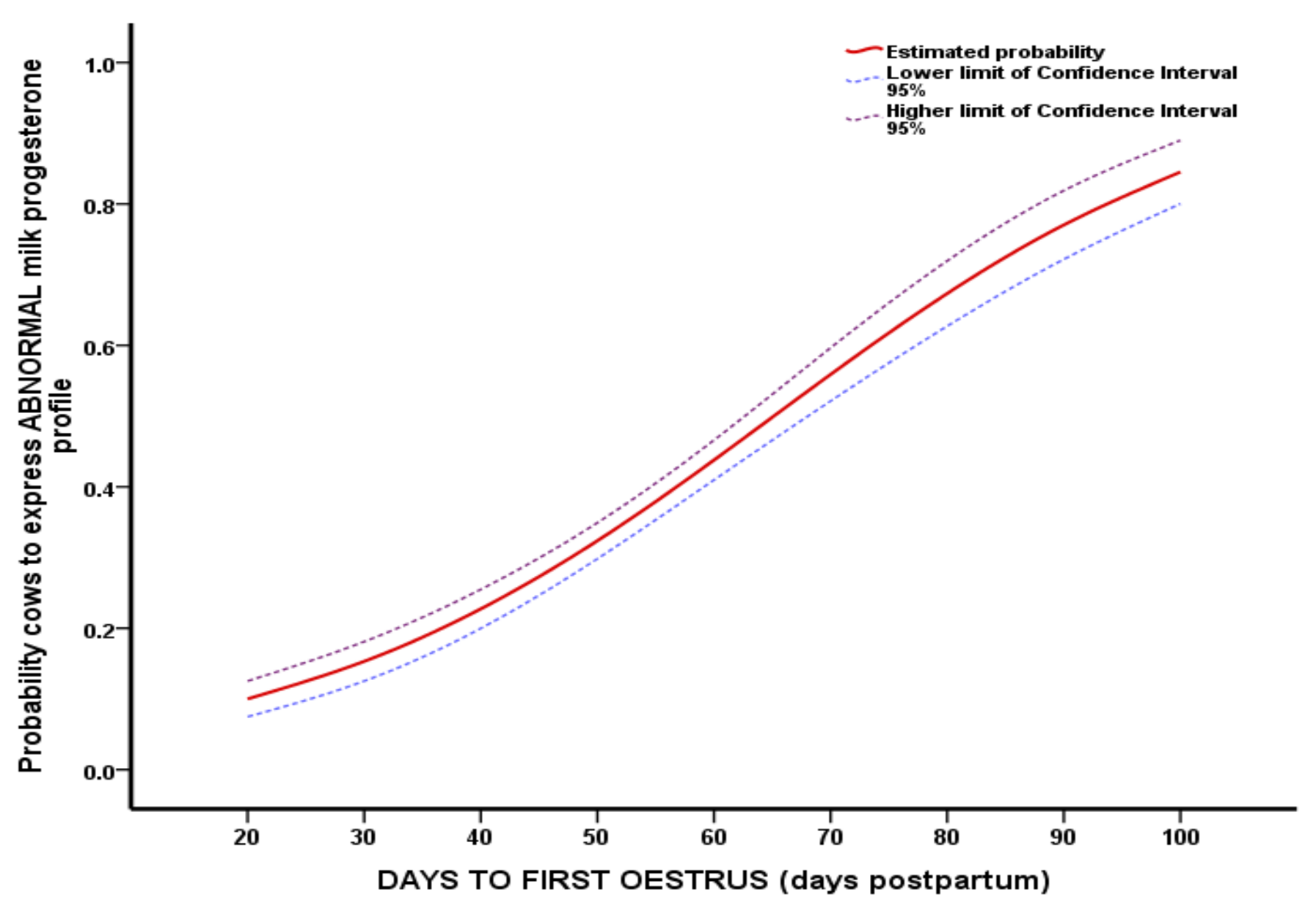

Figure 5.5: Effect of days to first oestrus on probability for cows to express abnormal milk progesterone profiles $(\mathrm{n}=56, P=0.016$, $\exp (b)=1.011$, CI for $\exp (b)$; [1.01, 1.09], beta coefficient $(b)=0.045, \mathrm{AUC}_{R O C}=0.72 \pm 0.07$, GLM with Bernoulli error distribution, logit link function, and robust standard error estimation). In this model, dependent variable was progesterone profile $(0=$ normal, $1=$ abnormal $)$ whereas days to oestrus and parity were added as continuous covariates. Red solid line represents the estimated probability whereas blue and purple dashed lines show the upper and lower $95 \%$ Confidence Interval (CI). 


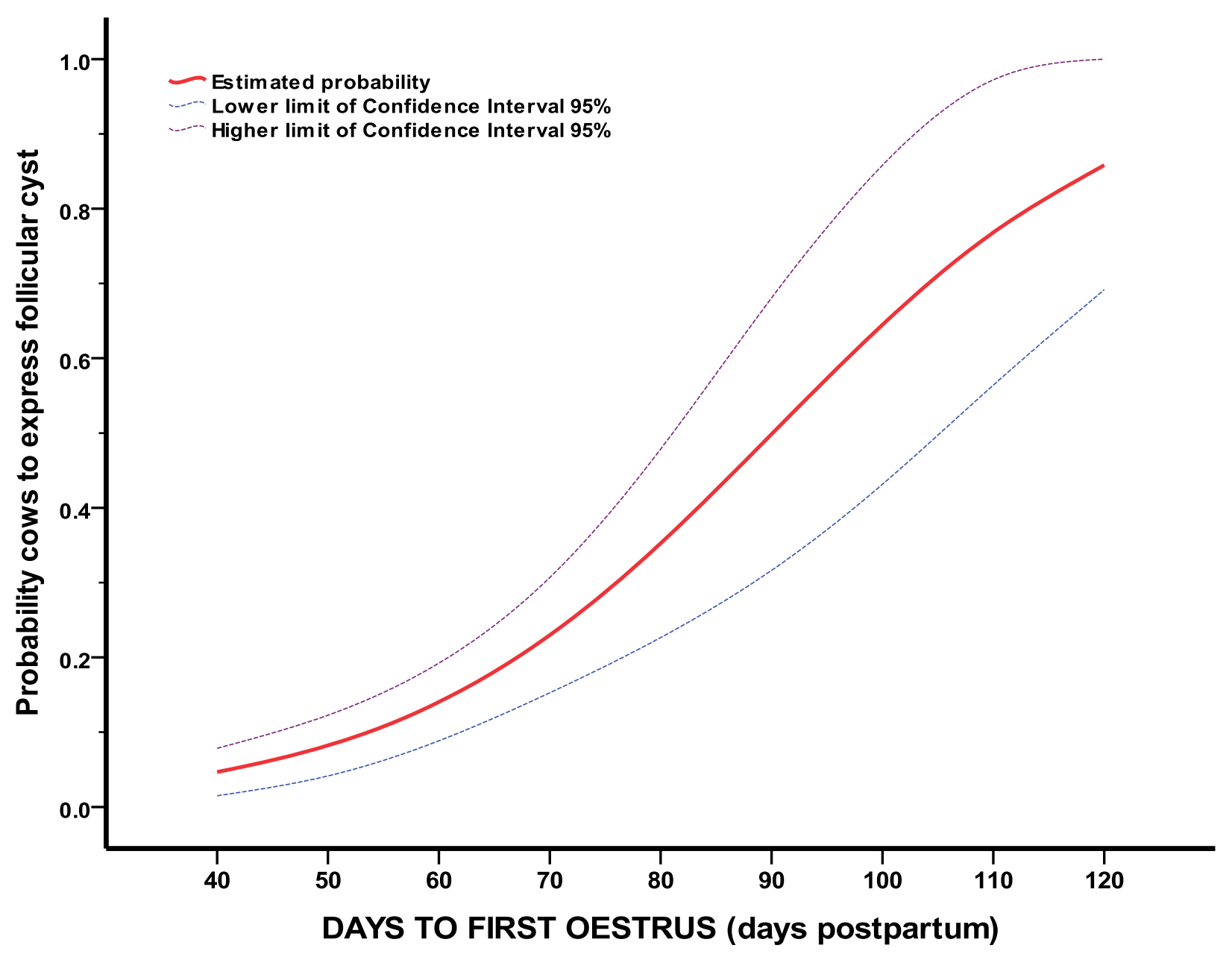

Figure 5.6: Effect of days to first oestrus on probability for cows to express cystic body $(\mathrm{n}=58, P=0.01$, exp $(b)=1.06$, CI for exp $(b)$; $[1.01,1.11]$, beta coefficient $(b)=0.06, \mathrm{AUC}_{R O C}=0.76 \pm 0.09$, GLM with Bernoulli error distribution, logit link function, and robust standard error estimation). In this model, dependent variable was cystic body $(0=$ cystic body was not expressed, $1=$ cystic body was expressed) whereas days to oestrus and parity were added as continuous covariates. Red solid line represents the estimated probability whereas blue and purple dashed lines show the upper and lower $95 \%$ Confidence Interval (CI). 


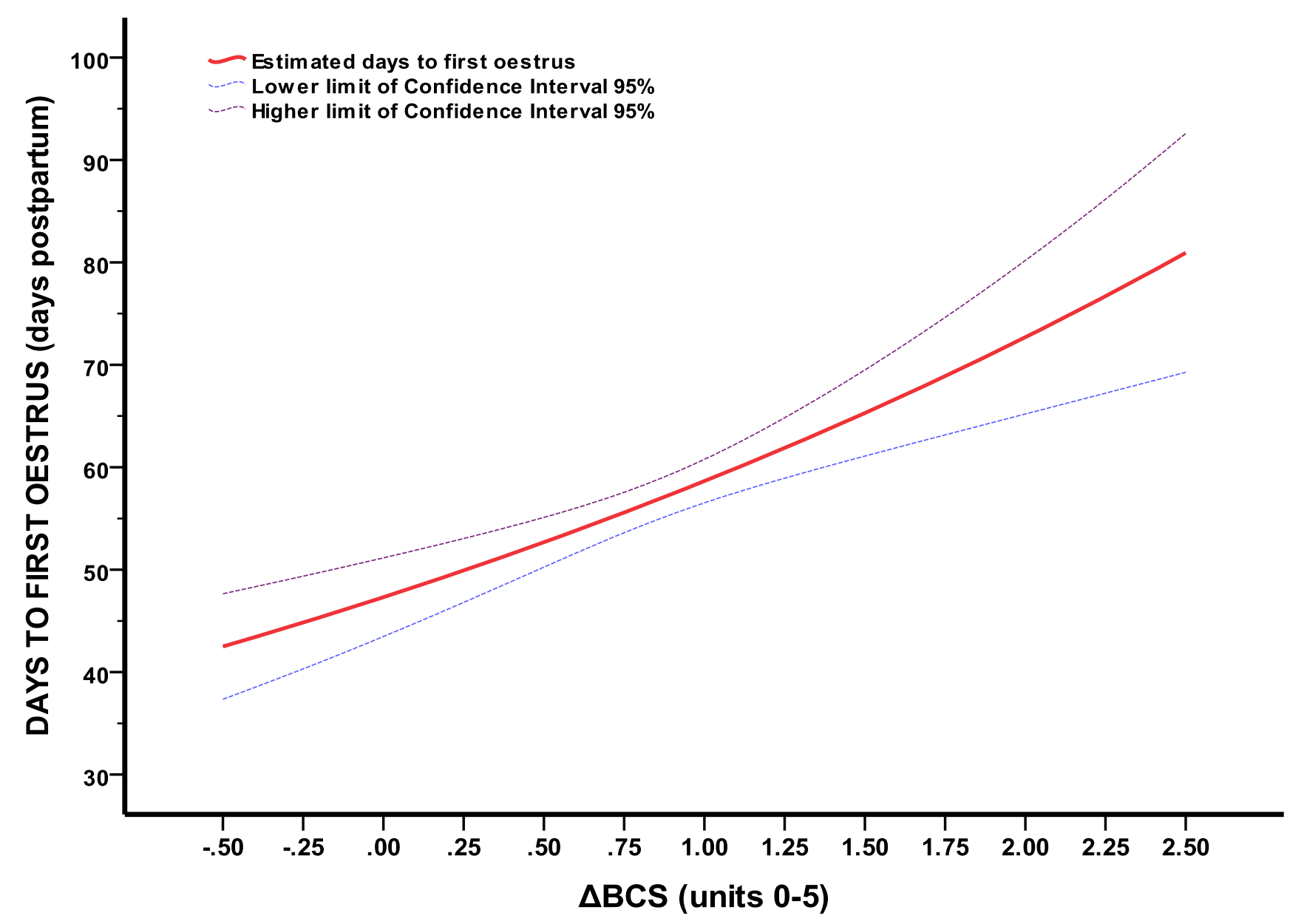

Figure 5.7: Effect of postpartum change in body condition score ( $\mathbf{A C C S})$ on days to first oestrus ( $\mathrm{n}=57, P=0.012)$. GLM with Poisson error distribution, $\log$ link function, and robust standard error estimation. In this model, dependent variable was days to first oestrus whereas $\triangle \mathrm{BCS}$ and parity were added as continuous covariates. Red solid line represents the estimated probability whereas blue and purple dashed lines show the upper and lower $95 \%$ Confidence Interval (CI). $\triangle \mathrm{BCS}$ values with negative sign indicate gain of BCS whereas positive $\triangle \mathrm{BCS}$ values denote loss of BCS. 


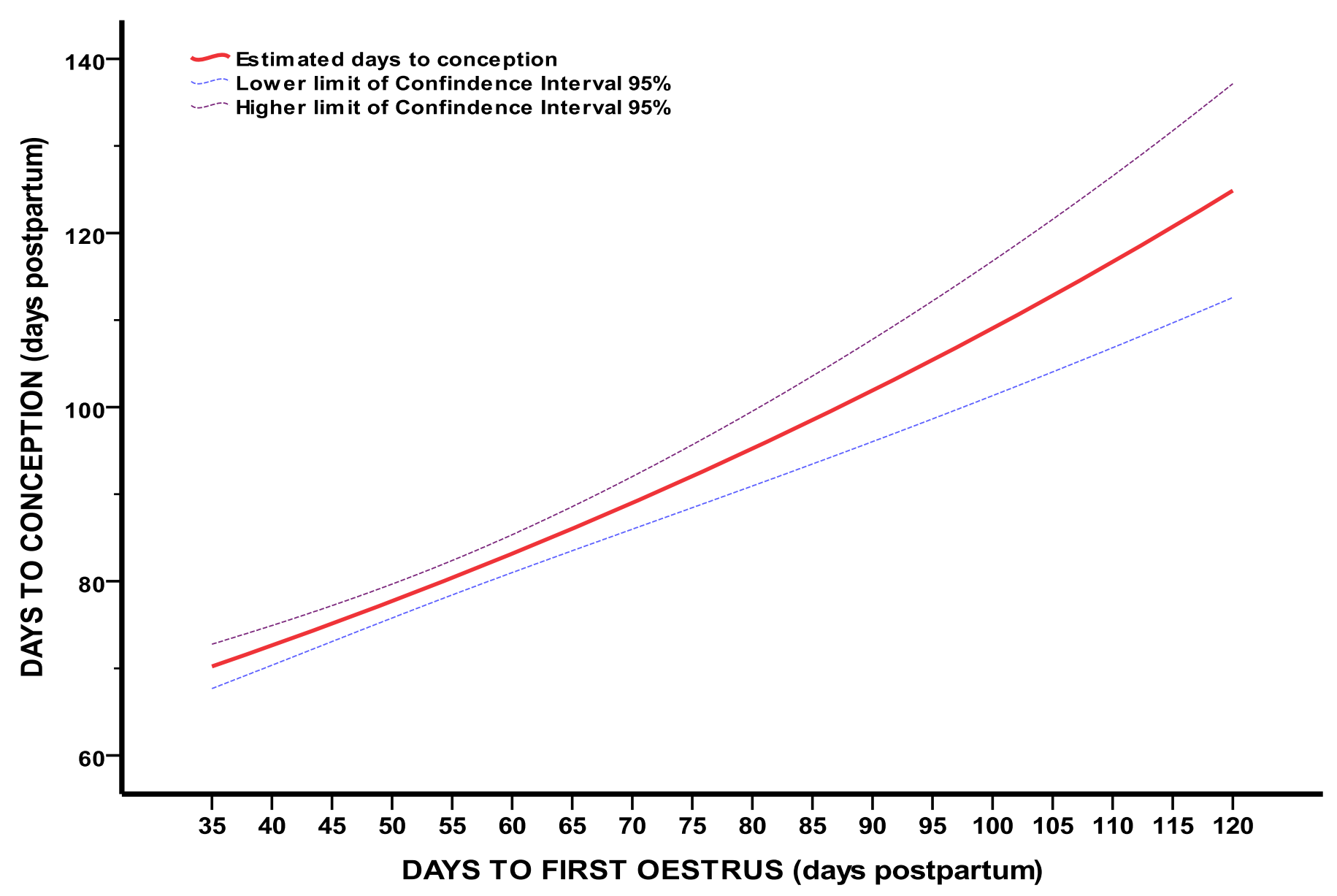

Figure 5.8: Effect of days to first oestrus on days to conception $(\mathrm{n}=21, P=0.001)$. GLM with Poisson error distribution, $\log$ link function, and robust standard error estimation. In this model, dependent variable was days to conception whereas days to first oestrus and parity were added as continuous covariates. Red solid line represents the estimated probability whereas blue and purple dashed lines show the upper and lower $95 \%$ Confidence Interval (CI). 


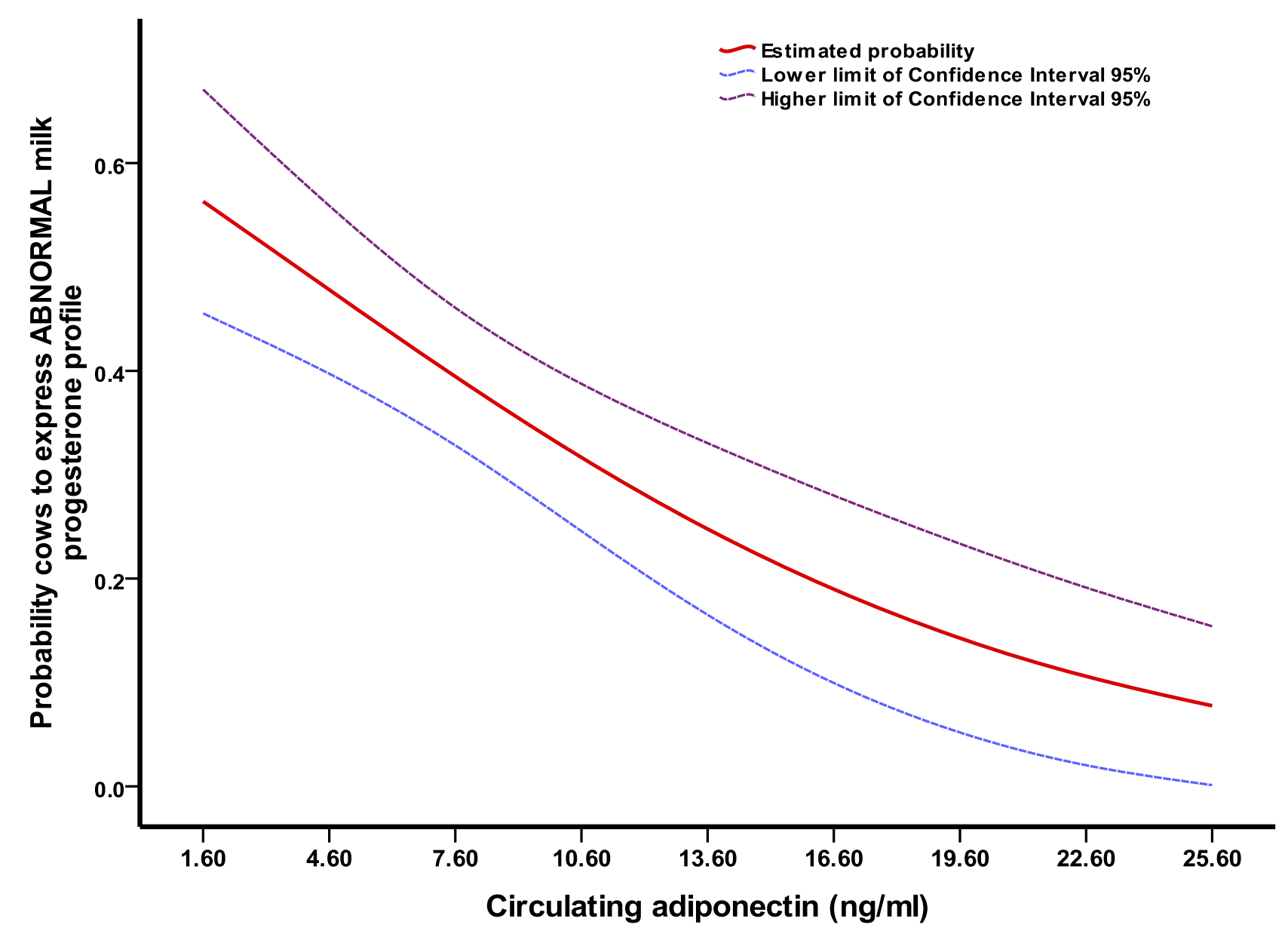

Figure 5.9: Effect of circulating adiponectin on probability for cows to express abnormal milk progesterone profiles $(\mathrm{n}=60, P=$ $0.037, \exp (b)=0.88$, CI for $\exp (b)$; $[0.79,0.99]$, beta coefficient $(b)=-0.12, \mathrm{AUC}_{R O C}=0.71 \pm 0.07$, GLM with Bernoulli error distribution, logit link function, and robust standard error estimation). In this model, dependent variable was progesterone profile $(0=$ normal, $1=$ abnormal) whereas circulating adiponectin and parity were added as continuous covariates. Red solid line represents the estimated probability whereas blue and purple dashed lines show the upper and lower $95 \%$ Confidence Interval (CI). 


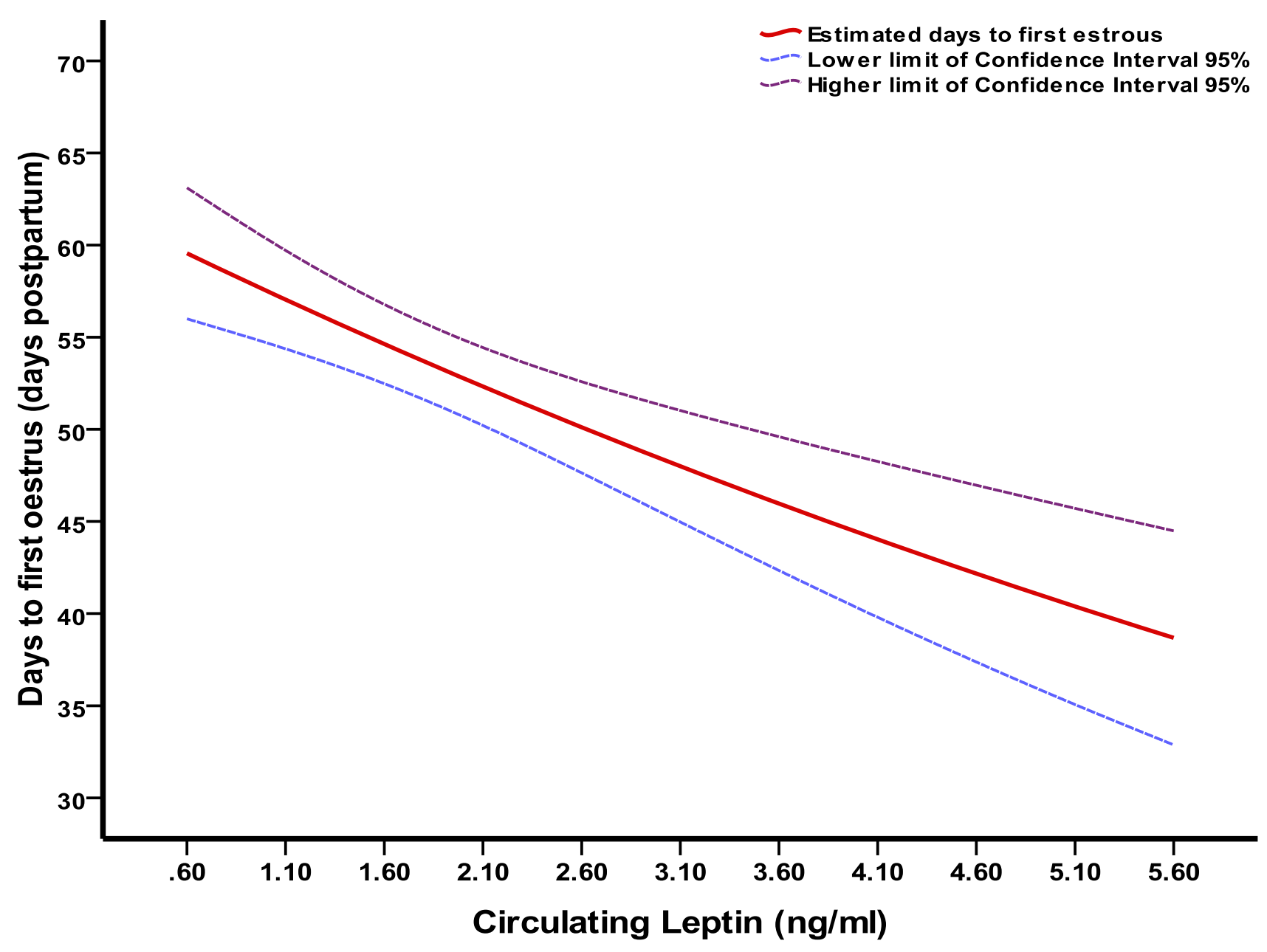

Figure 5.10: Effect of circulating leptin on days to first oestrus ( $\mathrm{n}=58, P=0.023)$, GLM with Poisson error distribution, $\log$ link function, and robust standard error estimation). In this model, dependent variable was days to first oestrus whereas circulating leptin and parity were added as continuous covariates. Red solid line represents the estimated probability whereas blue and purple dashed lines show the upper and lower $95 \%$ Confidence Interval (CI). 


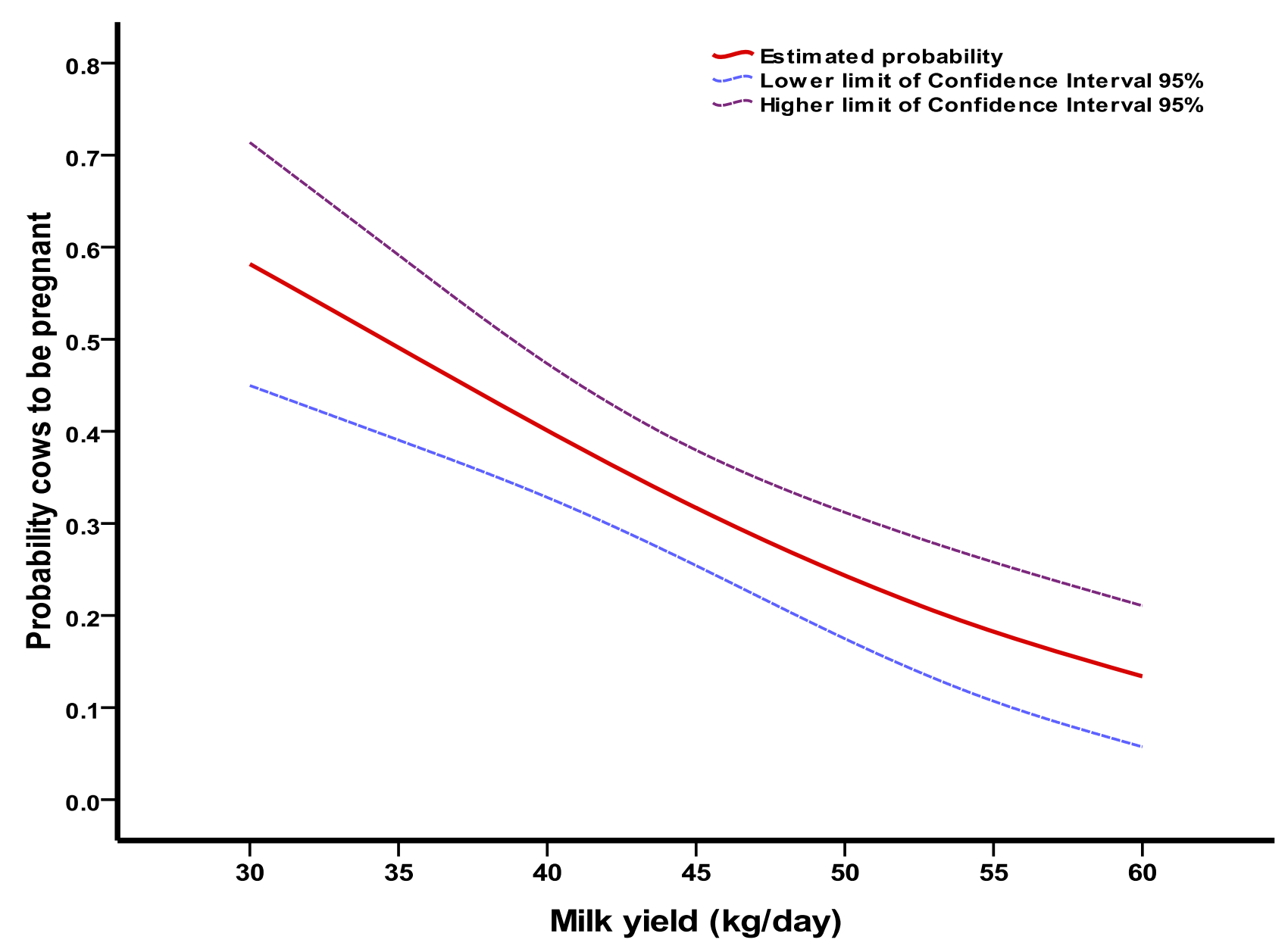

Figure 5.11: Effect of milk yield on probability of cows to be pregnant ( $\mathrm{n}=60, P=0.024$, exp $(b)=0.92$, CI for exp $(b) ;[0.86,0.98]$, beta coefficient $(b)=-0.08, \mathrm{AUC}_{R O C}=0.68 \pm 0.07$, GLM with Bernoulli error distribution, logit link function, and robust standard error estimation). In this model, dependent variable was pregnancy $(0=$ normal, $1=$ abnormal $)$ whereas milk yield and parity were added as continuous covariates. Red solid line represents the estimated probability whereas blue and purple dashed lines show the upper and lower $95 \%$ Confidence Interval (CI). 
Table 5.10: Effects of milk yield, DMI, GH, and parity on milk progesterone profile

\begin{tabular}{ccccccc}
\hline \hline & & \multicolumn{2}{c}{$\mathbf{9 5 \%}$ CI for exp $(\boldsymbol{b})$} & & & \\
\cline { 3 - 5 } Parameters in the model: & $\exp (\boldsymbol{b})^{\dagger}$ & Lower & Upper & & $\begin{array}{c}\text { Beta }(\boldsymbol{b}) \\
\text { coefficient }\end{array}$ & $\boldsymbol{P}$ \\
\hline Constant & - & - & - & -3.18 & 0.251 \\
Milk yield & 1.21 & 1.03 & 1.41 & 0.19 & 0.02 \\
DMI & 0.68 & 0.44 & 1.04 & -0.39 & 0.07 \\
GH & 1.31 & 0.97 & 1.76 & 0.27 & 0.07 \\
Parity & 1.86 & 0.91 & 3.75 & 0.62 & 0.09 \\
\hline \hline
\end{tabular}

Likelihood ratio test $\left(\chi^{2}=10.6, d f=4, P=0.031\right) ;$ Hosmer \& Lemeshow test for fitness $\left(\chi^{2}=12.6, d f=8, P=0.128\right) ; \quad \mathrm{AUC} C_{R O C}=0.73 \pm 0.07$

${ }^{\dagger} \exp (b)$ is the odds ratio

Table 5.11: Effects of insulin, glucose, GH, NEFA, and parity on follicular cyst formation

\begin{tabular}{|c|c|c|c|c|c|}
\hline \multirow[b]{2}{*}{ Parameters in the model: } & \multirow[b]{2}{*}{$\exp (b)^{\dagger}$} & \multicolumn{2}{|c|}{ 95\% CI for exp (b) } & \multirow[b]{2}{*}{$\begin{array}{c}\text { Beta }(b) \\
\text { coefficient }\end{array}$} & \multirow[b]{2}{*}{$\boldsymbol{P}$} \\
\hline & & Lower & Upper & & \\
\hline Constant & - & - & - & 16.84 & 0.012 \\
\hline Insulin & $\sim 0\left(2.410^{-11}\right)$ & 0.00 & 0.004 & -24.43 & 0.004 \\
\hline Glucose & 0.006 & 0.00 & 0.74 & -5.06 & 0.035 \\
\hline GH & 2.10 & 1.06 & 4.21 & 0.75 & 0.044 \\
\hline NEFA & 201.60 & 1.16 & 34,861 & 5.31 & 0.029 \\
\hline Parity & 0.96 & 0.31 & 2.90 & -0.04 & 0.944 \\
\hline
\end{tabular}

Likelihood ratio test $\left(\chi^{2}=25.3, d f=5, P=0.001\right) ;$ Hosmer \& Lemeshow test for fitness $\left(\chi^{2}=3.5, d f=8, P=0.90\right) ; \quad A U C_{R o c}=0.95 \pm 0.02$

${ }^{\dagger} \exp (b)$ is the odds ratio 
Table 5.12: Effects of insulin, milk yield, and IGF-I on days to conception

\begin{tabular}{ccccc}
\hline \hline & & \multicolumn{2}{c}{$\mathbf{9 5 \%}$ CI for $\boldsymbol{b}$} & \multirow{2}{*}{$\boldsymbol{P}$} \\
\cline { 3 - 5 } Parameters in the model: & $\begin{array}{c}\text { Beta }(\boldsymbol{b}) \\
\text { coefficient }\end{array}$ & Lower & Upper & 0.001 \\
\hline Constant & 3.65 & 3.210 & 4.096 & 0.001 \\
Insulin & 0.98 & .423 & 1.544 & 0.001 \\
Milk yield & 0.01 & .006 & .022 & 0.002 \\
IGF-I & -0.002 & -0.0034 & -0.0007 & \\
\hline \hline
\end{tabular}

Likelihood ratio test $\left(\chi^{2}=31.5, d f=4, P=0.001\right)$

Table 5.13: Model fitted values for different insulin concentrations

\begin{tabular}{|c|c|c|c|c|c|c|c|c|c|c|}
\hline $\begin{array}{l}\text { Insulin } \\
(n g / m l)\end{array}$ & 0.1 & 0.2 & 0.3 & 0.4 & 0.5 & 0.6 & 0.7 & 0.8 & 0.9 & 1.0 \\
\hline $\begin{array}{c}\text { Days to } \\
\text { conception } \\
\text { (days } \\
\text { postpartum) }\end{array}$ & $\begin{array}{c}57.4 \\
{[46.43,68.34]}\end{array}$ & $\begin{array}{c}63.3 \\
{[54.62,72.03]}\end{array}$ & $\begin{array}{c}69.9 \\
{[63.78,75.94]}\end{array}$ & $\begin{array}{c}77.1 \\
{[73.16,81.01]}\end{array}$ & $\begin{array}{c}85.1 \\
{[79.75,90.34]}\end{array}$ & $\begin{array}{c}93.8 \\
{[83.79,103.88]}\end{array}$ & $\begin{array}{c}103.5 \\
{[87.06,120.02]}\end{array}$ & $\begin{array}{c}114.2 \\
{[89.87,138.60]}\end{array}$ & $\begin{array}{c}126.1 \\
{[92.25,159.85]}\end{array}$ & $\begin{array}{c}139.1 \\
{[94.09,184.06]}\end{array}$ \\
\hline
\end{tabular}

${ }^{\dagger}$ Row is mean with $95 \% \mathrm{CI}$ 
Table 5.14: Effect of circulating adiponectin levels (ADP LEVELS) on metabolic hormones and metabolites

\begin{tabular}{|c|c|c|c|c|c|c|}
\hline \multirow{2}{*}{$\begin{array}{c}\text { Treatment: } \\
\text { Parameters: }\end{array}$} & \multicolumn{4}{|c|}{ ADP LEVELS } & \multirow[b]{2}{*}{ SED } & \multirow[b]{2}{*}{$P$} \\
\hline & NORMAL $^{\dagger}$ & $\boldsymbol{n}_{1}^{\curlyvee}$ & HIGH $^{\dagger}$ & $n_{2} \diamond$ & & \\
\hline Adiponectin $(n g / m l)$ & 7.12 & 346 & 21.32 & 41 & 1.328 & 0.001 \\
\hline Insulin $(n g / m l)$ & 0.40 & 337 & 0.42 & 40 & 0.043 & 0.730 \\
\hline Glucagon $(\mathrm{pg} / \mathrm{ml})$ & 95.2 & 178 & 96.6 & 38 & 9.38 & 0.885 \\
\hline $\mathbf{G H}(n g / m l)$ & 4.7 & 173 & 3.1 & 33 & 0.52 & 0.002 \\
\hline Leptin $(n g / m l)$ & 1.66 & 338 & 2.30 & 40 & 0.367 & 0.081 \\
\hline IGF-I $(n g / m l)$ & 118.7 & 167 & 154.9 & 30 & 14.78 & 0.015 \\
\hline Glucose $(\mathrm{mmol} / \mathrm{l})$ & 3.51 & 220 & 3.08 & 26 & 0.210 & 0.041 \\
\hline Urea $(\mathrm{mmol} / \mathrm{l})$ & 2.33 & 223 & 2.55 & 26 & 0.303 & 0.475 \\
\hline ВОНВ $(\mathrm{mmol} / \mathrm{l})$ & 0.66 & 223 & 0.53 & 26 & 0.070 & 0.093 \\
\hline NEFA $(\mathrm{mmol} / \mathrm{l})$ & 0.46 & 226 & 0.36 & 23 & 0.084 & 0.219 \\
\hline
\end{tabular}

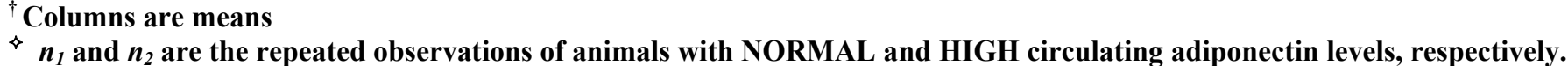

${ }^{\ddagger} \mathrm{SED}=$ standard error of the difference between treatment means

Table 5.15: Effect of circulating adiponectin levels (ADP LEVELS) on production traits

\begin{tabular}{|c|c|c|c|c|c|c|}
\hline \multirow{2}{*}{$\begin{array}{c}\text { Treatment: } \\
\text { Parameters: }\end{array}$} & \multicolumn{4}{|c|}{ ADP LEVELS } & \multirow[b]{2}{*}{ SED ${ }^{*}$} & \multirow[b]{2}{*}{$\boldsymbol{P}$} \\
\hline & NORMAL $^{\dagger}$ & $n_{1}^{\diamond}$ & HIGH $^{\dagger}$ & $n_{2} \diamond$ & & \\
\hline DMI $(\mathrm{kg} / \mathrm{d})$ & 21.7 & 316 & 19.5 & 36 & 0.99 & 0.028 \\
\hline Milk Yield $(\mathrm{kg} / \mathrm{d})$ & 44.1 & 316 & 38.2 & 36 & 2.17 & 0.01 \\
\hline LWT $(k g)$ & 657.1 & 316 & 659.3 & 36 & 7.74 & 0.776 \\
\hline BCS at calving & 3.45 & 54 & 3.13 & 6 & 0.134 & 0.017 \\
\hline MEBAL $(M J / d)$ & -5.4 & 316 & -6.2 & 36 & 2.18 & 0.697 \\
\hline$\Delta \mathbf{B C S}$ (units 1-5) & 0.70 & 53 & 0.25 & 6 & 0.185 & 0.015 \\
\hline
\end{tabular}

\footnotetext{
${ }^{\dagger}$ Columns are means

$\diamond n_{1}$ and $n_{2}$ are the repeated observations of animals with NORMAL and HIGH circulating adiponectin levels, respectively.

${ }^{*} \mathrm{SED}=$ standard error of the difference between treatment means
} 


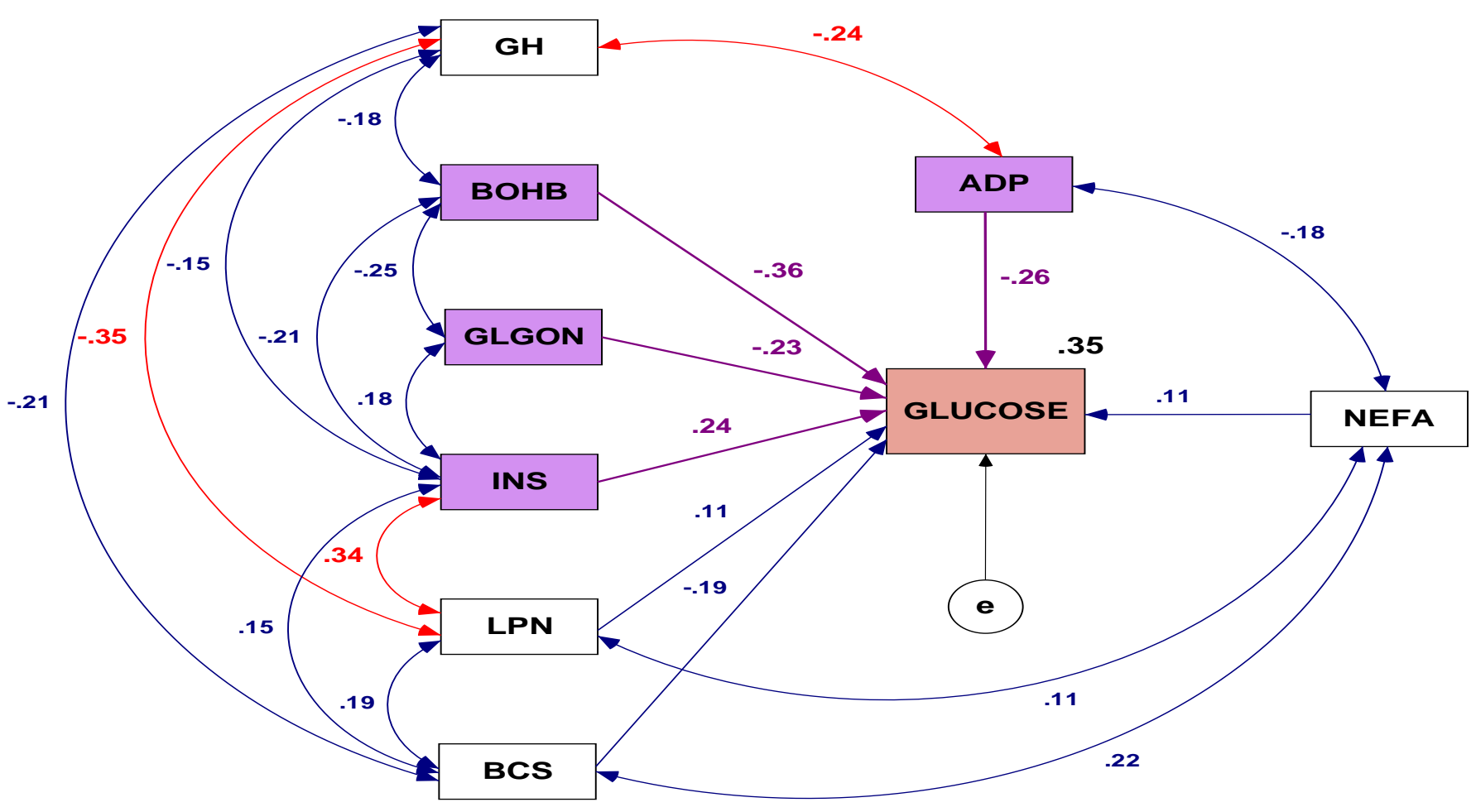

Figure 5.12: The basic model: Glucose interrelationships with adiponectin, BCS, metabolites, and metabolic hormones $\left(n=60 ; \chi^{2}=5.9, d f\right.$ $=15, P=0.981$; Bollen-Stine nonparametric bootstrap $P=0.954, \mathrm{~N}=1,000$ samples; $\mathrm{CFI}=1.00$; NFI=0.918; RMSEA=0.0001; AIC=83.51; DIC=86.02, $P=0.62$, Effective number of parameters $=33.32$, convergence was achieved after $(68,500) * 2$ burn-outs, 500 samples per each burn-in, convergence cut-off limit 1.002). In this basic model, single headed arrows $(\rightarrow)$ represent effects of BCS, metabolites, and metabolic hormones on glucose, and they are quantified as standardized regression coefficients placed in the middle of the arrows. Double headed arrows represent partial correlations and their coefficients are approximately located in the middle of arrow curvature. Purple single headed arrows $(\rightarrow)$ and red double headed arrows ( $\leftarrow$ ) mark significant effects or correlations $(P<0.05)$, whereas dark blue single headed arrows $(\rightarrow)$ and dark blue double headed arrows $(\leftrightarrow)$ mark nonsignificant effects or correlations. Thus, the correlations INS $\leftrightarrow$ LPN $(0.34, P=0.012)$, LPN $\leftrightarrow$ GH $(-0.35, P=0.01)$, and GH↔ADP $(-0.24, P=0.042)$ and the effects $(\rightarrow)$ of INS $(0.24, P=0.036)$, GLGON $(-0.23, P=0.036)$, BOHB $(-0.36, P=0.001)$, and ADP $(-0.26, P=0.014)$ on glucose are significant. Partial correlation coefficients and standardized regression coefficients are interpreted the same, as classical correlation coefficients. Model squared multiple correlations coefficient (which is equal to adjusted $R^{2}$ of linear regression models) is 0.35 . Variable $e$ is the residual error of the model. Model parameter estimates (mean $\pm \mathrm{SE})$ are illustrated in Table B.1 in appendix. 


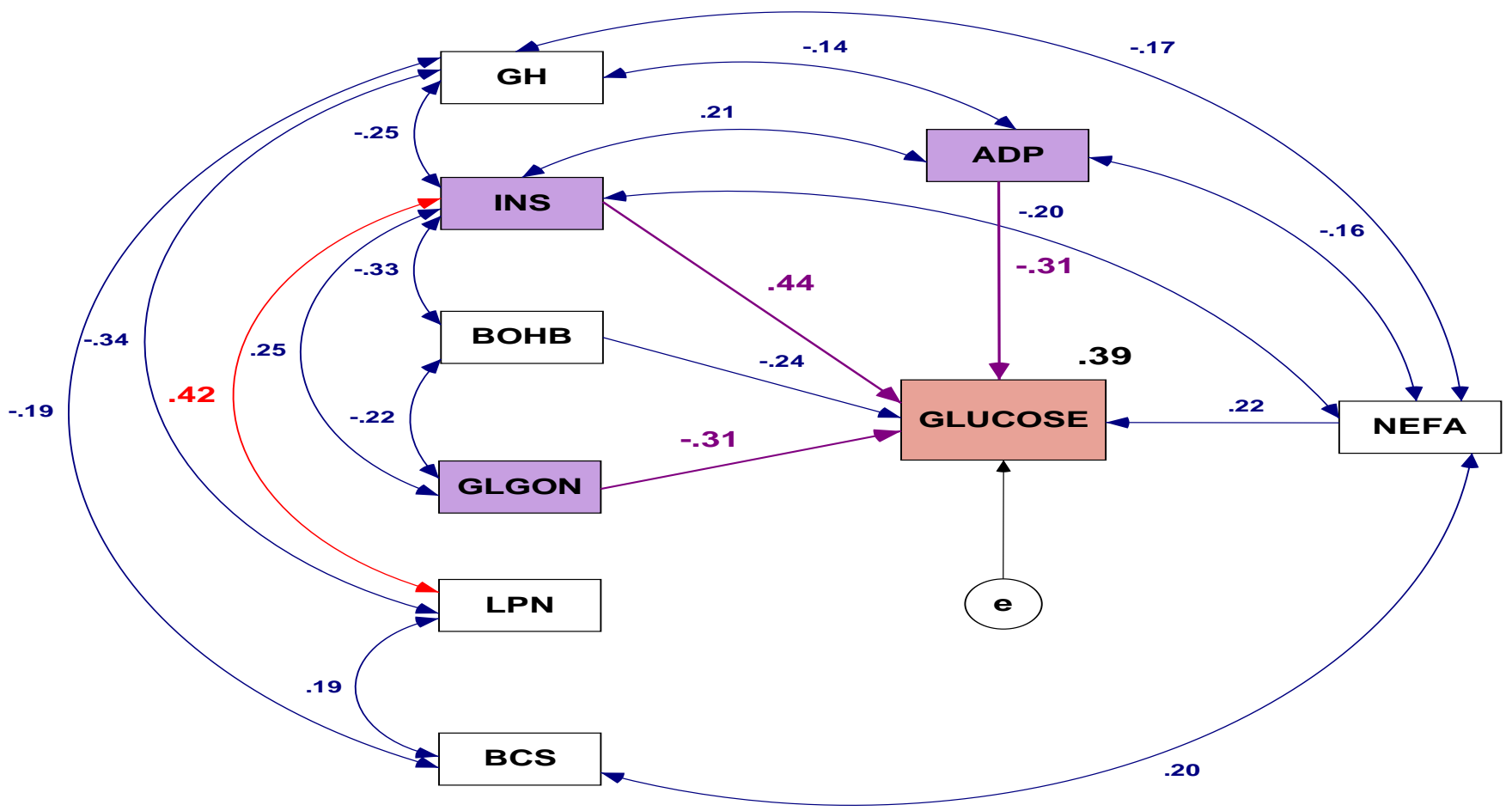

Figure 5.13: Glucose interrelationships with adiponectin, BCS, metabolites, and metabolic hormones in cows with LOW previous milk production $\left(n=30 ; \chi^{2}=5.9, d f=17, P=0.996 ; \mathrm{CFI}=1.00 ; \mathrm{NFI}=0.865 ; \mathrm{RMSEA}=0.0001 ; \mathrm{AIC}=79.86\right.$; DIC=83.17, $P=0.56$, Effective number of parameters $=24.18$, convergence was achieved after $(77,500) * 4$ burn-outs, 500 samples per each burn-in, convergence cut-off limit 1.002$)$. In this model, single headed arrows represent $(\rightarrow$ ) effects of BCS, metabolites, and metabolic hormones on glucose and they are quantified as standardized regression coefficients placed in the middle of the arrows. Double headed arrows represent partial correlations and their coefficients are approximately located in the middle of arrow curvature. Purple single headed arrows $(\rightarrow)$ and red double headed arrows $(\leftrightarrow)$ mark significant effects or correlations $(P<0.05)$, whereas dark blue single headed arrows $(\rightarrow)$ and dark blue double headed arrows $(\leftrightarrow)$ mark non-significant effects or correlations. Thus, the correlations INS $\leftrightarrow$ LPN $(0.42, P=0.022)$ and the effects $(\rightarrow)$ of INS $(0.44, P=0.008)$, GLGON $(-0.31, P=0.043)$, and ADP $(-$ $0.31, P=0.041)$ on glucose are significant. Partial correlation coefficients and standardized regression coefficients are interpreted the same, as classical correlation coefficients. Model squared multiple correlations coefficient (which is equal to adjusted $R^{2}$ of linear regression models) is 0.39 . Variable $e$ is the residual error of the model. Model parameter estimates (mean $\pm \mathrm{SE}$ ) are illustrated in Table B.1 in appendix. 


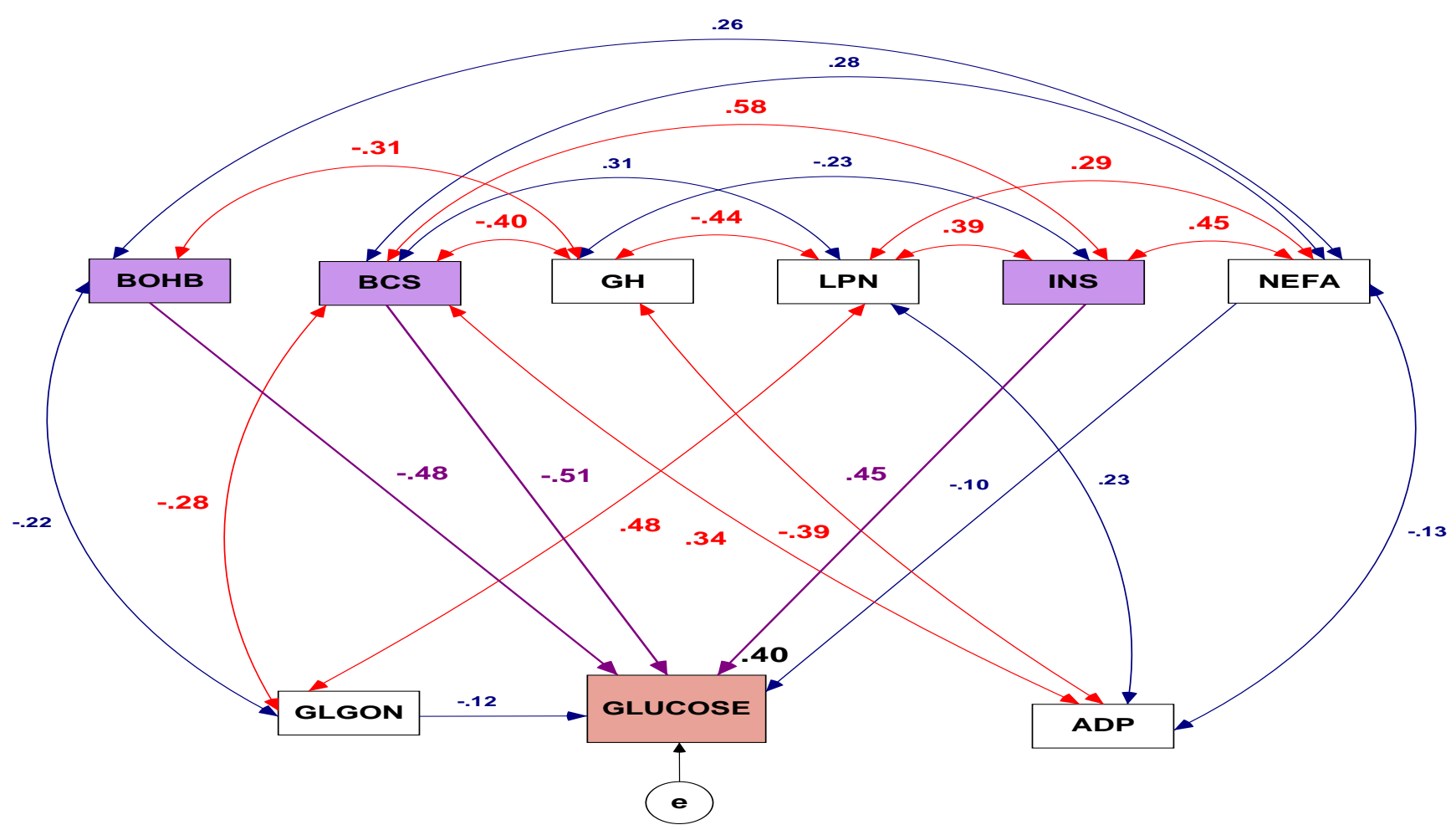

Figure 5.14: Glucose interrelationships with adiponectin, BCS, metabolites, and metabolic hormones in cows with HIGH previous milk production $\left(n=30 ; \chi^{2}=3, d f=13, P=0.998 ; \mathrm{CFI}=0.998\right.$; NFI=0.966; RMSEA $=0.001 ; \mathrm{AIC}=85.01$; DIC $=88.29$, $P=0.43$, Effective number of parameters $=23.34$, convergence was achieved after $(89,500) * 4$ burn-outs, 500 samples per each burn-in, convergence cut-off limit was 1.002$)$. In this model, single headed arrows $(\rightarrow$ ) represent effects of BCS, metabolites, and metabolic hormones on glucose and they are quantified as standardized regression coefficients placed in the middle of the arrows. Double headed arrows represent partial correlations and their coefficients are approximately located in the middle of arrow curvature. Purple single headed arrows $(\rightarrow)$ and red double headed arrows $(\leftrightarrow)$ mark significant effects or correlations $(P<0.05)$, whereas dark blue single headed arrows $(\rightarrow)$ and dark blue double headed arrows $(\leftrightarrow)$ mark non-significant effects or correlations. Thus, the correlations of INS $\leftrightarrow$ LPN $(0.39, P=0.02)$, INS $\leftrightarrow$ BCS $(0.58, P=0.003)$, INS $\leftrightarrow$ NEFA $(0.45, P=0.02)$, LPN $\leftrightarrow$ NEFA( 0.29 , $P=0.05), \mathrm{LPN} \leftrightarrow \mathrm{GLGON}(0.48, P=0.004), \mathrm{LPN} \leftrightarrow \mathrm{GH}(-0.44, P=0.01), \quad \mathrm{BCS} \leftrightarrow \mathrm{GH}(-0.40, P=0.027), \mathrm{GH} \leftrightarrow \mathrm{BOHB}(-0.31, P=0.05), \mathrm{GH} \leftrightarrow \mathrm{ADP}(-0.39$, $P=0.036)$, GLUGON $\leftrightarrow \mathrm{BCS}(-0.28, P=0.049)$, and $\mathrm{ADP} \leftrightarrow \mathrm{BCS}(0.34, P=0.031)$, and the effects $(\rightarrow)$ of INS $(0.45, P=0.035), \mathrm{BCS}(-0.51, P=0.01)$, and BOHB $(-0.48, P=0.001)$ on glucose are significant. Partial correlation coefficients and standardized regression coefficients are interpreted the same, as classical correlation coefficients. Model squared multiple correlations coefficient (which is equal to adjusted $R^{2}$ of linear regression models) is 0.40 . Variable $e$ is the residual error of the model. Model parameter estimates (mean $\pm \mathrm{SE}$ ) are illustrated in Table B.1 in appendix. 


\subsection{DISCUSSION}

\subsubsection{Effect of circulating adiponectin levels on metabolic hormones, metabolites, productive traits, and reproductive performance}

Raddatz et al., (2008) reported that individual cows had consistently high or low adiponectin levels throughout the sampling period and this result is in agreement with the present study. Moreover, the present study found that the group of the cows showed persistently HIGH adiponectin levels had 3 times higher circulating adiponectin than cows with consistently NORMAL adiponectin levels. The present study for first time, to the best of our knowledge, analyzes the effects of differences observed in circulating adiponectin levels in dairy cows. These differences were associated with differences in metabolic, hormonal, productive and reproductive traits. Unfortunately, only $10 \%$ of cows in this experiment expressed HIGH adiponectin levels. This point was a drawback in the statistical analysis because the data were unbalanced and this limits the conclusions. Nevertheless, large differences were observed in metabolic, hormonal, productive and reproductive traits, and different patterns in metabolic and reproductive regulation were demonstrated between cows expressing NORMAL and HIGH adiponectin levels.

In the present study, cows with HIGH adiponectin levels had lower GH and glucose than cows with NORMAL adiponectin levels. Moreover, cows with HIGH adiponectin levels had trends for higher circulating leptin and BOHB than cows with NORMAL adiponectin levels. Cows with HIGH adiponectin levels ate less feed and produced less milk than cows with NORMAL adiponectin profiles. Finally, cows with HIGH adiponectin levels lost less BCS postpartum, and had lower BCS at calving than cows with NORMAL adiponectin levels. Studies in humans and rodents indicate that the relation between adiponectin and GH is negative (Lam et al., 2004; Nilsson et al., 2005; Rodriguez-Pacheco et al., 2007). In accordance with this, cows with HIGH adiponectin levels had lower GH than cows with NORMAL adiponectin levels. It is known that GH has rapid direct catabolic actions (enhances lipolysis, decreases lipogenesis and restricts glucose transport) whereas 
adiponectin has insulin-sensitizing actions (decreases glucose output, increases FFA oxidation, and increases influx of NEFA) (Davidson, 1987; Lucy, 2000). Thus, low GH and high adiponectin in cows with HIGH adiponectin levels may explain why these animals had lower glucose and a trend for lower BOHB.

In humans, acromegalic and anorexia nervosa patients expressed decreased circulating leptin and increased circulating GH (Popovic et al., 2001; Scacchi et al., 1999) and this may indicate the relationship between $\mathrm{GH}$ and leptin is negative at least in extreme conditions with elevated circulating GH. Also, Leifers et al. (2005) suggested that leptin expression in adipose tissue is possibly regulated in early lactating cows by the negative impact of GH. Block et al. (2001) also demonstrated that the correlation between GH and leptin is negative. Moreover, restriction of energy intake suppresses GH secretion in rodents, but stimulates it in humans and ruminants (Block et al., 2001; Nagatani et al., 2000). In line with these results, cows with HIGH adiponectin levels in this experiment had low GH and DMI, and a trend for high leptin. It is known that the main central effect of leptin is suppression of food intake (Chilliard et al., 2005), and this may explain why cows with HIGH adiponectin levels in this experiment had lower DMI than cows with NORMAL adiponectin levels. Furthermore, leptin stimulates free fatty acids (FFA) oxidation and increases glucose uptake in the skeletal muscles, and decreases glucose output and increases FFA oxidation in the liver (Yildiz \& Haznedaroglu, 2006; Kokta et al., 2004; Macajova et al., 2004). This may provide further explanation why glucose was low, and BOHB had a trend to be low in cows with HIGH adiponectin levels. In the present study, cows with HIGH adiponectin levels had higher adiponectin and a tendency for higher leptin than cows with NORMAL adiponectin levels. Some studies suggest that the relationship between adiponectin and leptin is negative (Matsubara et al., 2002; Huypens, 2007). However, other studies reported positive correlations between leptin and adiponectin (Pardo et al., 2004; Rossi et al., 2005) or no correlation (Park et al., 2004). However, in acromegalic patients GH down-regulated both circulating adiponectin and leptin (Popovic et al., 2001; Lam et al., 2004). Thus, at least in extreme conditions, it is possible for adiponectin and leptin to be positively correlated, especially when circulating GH is very high or very low. In the present study, cows with HIGH adiponectin levels had 
approximately $34 \%$ lower circulating GH compared with cows with NORMAL adiponectin levels, and maybe that is the reason why cows with HIGH adiponectin levels had higher circulating adiponectin and a trend for higher leptin than cows with NORMAL adiponectin levels. TNF- $\alpha$ is another interesting adipokine and it has been found to exert regulatory roles on adiponectin and leptin. Classically, TNF- $\alpha$ is increased in periparturient cows and decreased in early lactating cows (Ametaj, 2005). Also, TNF- $\alpha$ is a potent inhibitor of adiponectin expression and secretion by adipose tissue, and negatively correlated with insulin sensitivity (Fasshauer et al., 2002; Ruan et al., 2002; Ronti et al., 2006). Moreover, TNF- $\alpha$ has been demonstrated to stimulate leptin (Zumbach et al., 1997) in humans. Unfortunately, the present study did not measure TNF- $\alpha$, but it is possible that this adipokine is the link that explains the high circulating leptin and adiponectin observed in cows with HIGH adiponectin levels.

In early lactating cows, low circulating insulin is responsible for uncoupling of the GH IGF-I axis in the liver due to down-regulation of GH receptors (Lucy; 2000). Consequently, IGF-I production in the liver is suppressed and the negative feedback of IGF-I at the level of the hypothalamus/ pituitary is removed, resulting in increased circulating GH. This situation can be restored by improving insulin sensitivity (Wathes et al., 2007; Garnsworthy et al., 2008a; Leroy et al., 2010). Cows with HIGH adiponectin levels had higher circulating IGF-I, but lower circulating GH than cows with NORMAL adiponectin levels in this experiment. However, circulating insulin did not differ between the two groups. In line with this experiment, animals with low IGF-I have greater circulating GH (Lucy, 2000). Elevated circulating GH can be the main reason for lower circulating IGF-I in cows with NORMAL adiponectin levels due to uncoupling of the GHIGF-I axis in the liver of these animals. It is known that IGF-I stimulates the synthesis of adiponectin (Williams et al., 2004) and that may explain why cows with HIGH adiponectin levels had elevated adiponectin and IGF-I. Moreover, the combination of high circulating adiponectin and leptin along with decreased glucose and the same levels of circulating insulin in cows with HIGH adiponectin levels may imply better insulin sensitivity of these animals. High insulin sensitivity is associated with weight gain, and accretion of condition is positively associated with circulating IGF-I (Wathes et al., 2007). IGF-I concentrations 
were positively correlated with insulin sensitivity in humans (Sesti et al., 2005) and that might be another reason why circulating IGF-I was higher in cows with HIGH adiponectin levels.

According to Meikle et al. (2004) leptin and IGF-I are positively correlated in periparturient dairy cows and this is in agreement with this study. Also, the lower circulating glucose for the same levels of circulating insulin in cows with HIGH adiponectin levels might suggest better response of insulin to glucose and thus better insulin sensitivity than cows with NORMAL adiponectin levels. Low insulin sensitivity is common in high yielding cows (Chilliard et al., 1998; Gutierrez et al., 2006). High GH concentrations not only stimulate milk production, but also stimulate glucose production in the liver, and adipose tissue mobilization. The resulting high circulating NEFA, BOHB, and GH inhibit insulin action and create a further state of peripheral insulin resistance (Leroy et al., 2010). Furthermore, milk yield and IGF-I are negatively correlated (Wathes et al., 2007). In the present study, cows with NORMAL adiponectin levels had higher milk yield and greater BCS loss postpartum than cows with HIGH adiponectin levels and this also agrees with the lower circulating levels of IGF-I in cows with NORMAL adiponectin levels.

Differences in productive traits were generally matched by differences in metabolic and hormonal profile in this experiment. Cows with HIGH adiponectin levels had lower DMI and $\triangle \mathrm{BCS}$, and produced lower milk yield than cows with NORMAL adiponectin levels. The lower DMI in cows with HIGH adiponectin levels may be was the result of elevated circulating leptin in these animals (as increased leptin suppresses feed intake). In addition, decreased circulating GH and glucose in cows with HIGH adiponectin levels may be was the main reason for decreased milk yield and decreased $\triangle \mathrm{BCS}$. It is known that fatter cows at calving lose more body condition than thinner cows (Garnsworthy \& Topps, 1982), and this was also true in the present study because cows with HIGH adiponectin levels were thinner at calving and they lost less condition throughout the experiment compared to cows with NORMAL adiponectin levels. Moreover, BOHB had a tendency to be elevated in 
cows with NORMAL adiponectin levels and that indicates greater dependence on fat tissue mobilization for supporting increased milk yield.

Reproductive functions are closely related to nutritional status (Boland et al., 2001; Garnsworthy et al., 2008; Santos, 2007). Adipokines such as leptin and adiponectin are known to be implicated in reproductive functions at different levels, including central effects on the hypothalamus and pituitary, peripheral effects on the ovary and reproductive tract, and direct effects on the oocyte and embryo (Mitchell et al., 2005). In the present study, cows with HIGH adiponectin levels showed higher probability to become pregnant than cows with NORMAL adiponectin levels. Moreover, cows with NORMAL milk progesterone profile were more likely to be pregnant than cows with ABNORMAL milk progesterone profile, and there was a tendency for non-random association between cows expressing HIGH circulating adiponectin levels and cows with NORMAL milk progesterone profile. This may explain in part the higher pregnancy rate in cows with HIGH adiponectin levels, but it needs to be investigated further.

The present study showed that the association of $\triangle \mathrm{BCS}$ with the probability of a cow to be pregnant was non linear. Furthermore, cows with HIGH adiponectin levels lost less BCS (0.25 units versus 0.70 units) than cows with NORMAL adiponectin levels. Moreover, cows losing 0.25 units of BCS or less were more likely to become pregnant than cows losing 0.70 units or more (Figure 5.3). This is another explanation for the higher pregnancy rate in cows with HIGH adiponectin levels (Table 5.7). Also, the association of $\triangle \mathrm{BCS}$ with days to first oestrus was negative (Figure 5.7) in the present study and this may explain why cows with HIGH adiponectin levels had fewer days to first oestrus than cows with NORMAL adiponectin levels (Table 5.7).

IGF-I was associated with the probability for a cow to become pregnant in this experiment. Cows with HIGH adiponectin levels had higher circulating IGF-I than cows with NORMAL adiponectin levels. Cows with average circulating IGF-I equal to or greater than $155 \mathrm{ng} / \mathrm{ml}$ throughout the experimental period were more likely to become pregnant than cows with average circulating IGF-I equal to or less than $120 \mathrm{ng} / \mathrm{ml}$. Also, milk yield was 
negatively associated with the probability of cows to be pregnant in the present study. These may further explain the higher pregnancy rate in cows with HIGH adiponectin levels.

Cows with HIGH adiponectin levels had fewer days to first oestrus than cows with NORMAL adiponectin levels, and days to first oestrus were positively associated with the probability of cows to express ABNORMAL milk progesterone. Also, circulating adiponectin was negatively associated with the probability of cows to express ABNORMAL milk progesterone profile. Taken together these findings may explain why in this experiment there was a tendency for non-random association between cows expressed HIGH circulating adiponectin levels and cows with NORMAL milk progesterone profile. According to Dupont et al. (2008) adiponectin activates AMPK and that leads to decreased steroidogenesis in the granulosa cells. Indeed, in vitro studies showed a possible role of adiponectin in modulating ovarian steroidogenesis (Ledoux et al., 2006; Lagaly et al., 2008; Chabrolle et al., 2009; Gutman et al., 2009; Pierre et al., 2009; Maillard et al., 2010), but results are controversial, which may imply speciesspecific differences in the regulation of steroidogenesis by adiponectin (Dupont et al., 2008; Brochu-Gaudreau et al., 2010).. Moreover, women treated with chorionic gonadotrophin expressed increased circulating adiponectin, which correlated with circulating progesterone and oestradiol (Liu et al., 2006). Furthermore, short-term treatment of rat pituitary gonadotroph cells with recombinant murine adiponectin abolishes GnRH-induced LH secretion (Rodriguez-Pacheco et al., 2007). Adiponectin has been detected in porcine and human follicular fluid (Ledoux et al., 2006; Chabrolle et al., 2009) and AdipoR1 and AdipoR2 are present in theca and granulosa cells, oocytes, and the corpus luteum in dairy cows (Tabandeh et al., 2010). However, either AdipoR1 or AdipoR2 knock-out mice is fertile, and this may imply adiponectin is not necessary for normal ovarian function (Campos et al., 2008; Brochu-Gaudreau et al., 2010). The effect of adiponectin on milk progesterone profile needs to be investigated further.

Treatment with leptin reverses fasting-induced anoestrus in Syrian hamsters (Schneider et al., 1998). Moreover, female mice injected with leptin reproduced up to 9 days earlier than 
controls and showed earlier maturation of the reproductive tract (Chehab et al., 1997). Furthermore, higher leptin concentrations were associated with shorter intervals to first observed estrus in dairy cows (Liefers et al., 2003). Leptin was negatively associated with days to first oestrus in this experiment, which is in line with the afore-mentioned studies. Moreover, cows with HIGH adiponectin levels had lower days to first oestrus and a tendency for higher leptin than cows with NORMAL adiponectin levels. Thus, the tendency for higher leptin may explain the lower days to first oestrus in cows with HIGH adiponectin levels.

In lactating cows, delayed resumption of cyclicity is associated with decreased circulating leptin, whereas increased circulating leptin resulted in improved reproductive performance (Kadokawa et al., 2000; Block et al., 2001; Liefers et al., 2003; Meikle et al., 2004; Konigsson et al., 2008). According to Meikle et al. (2004) cows with better reproductive performance had higher circulating IGF-I and Butler et al. (2000) found a negative relationship between postpartum circulating IGF-I and the interval to the resumption of ovarian cyclicity. Also, Huszenicza et al. (2001) found higher IGF-I levels in cows with ovulation occurring within 35 days postpartum. In the present study, cows with HIGH adiponectin levels had higher IGF-I, leptin (tendency), and reproductive performance than cows with NORMAL adiponectin profiles and those findings are generally in agreement with the above mentioned studies.

It is known that cows with high genetic merit show increased incidence of delayed commencement of luteal activity (CLA) (Windig et al., 2008) and increased circulating GH and milk yield (Veerkamp et al., 2003). Moreover, cows with greater milk production have lower peak progesterone concentrations (Windig et al., 2008; Taylor et al., 2003; Remppis et al., 2011) and secretion of progesterone is reduced by energy deficit (VillaGodoy et al., 1988). According to Veerkamp et al. (2000) increasing genetic merit for feed intake improves CLA in dairy cows. Moreover, cows in higher parities are more at risk of suffering from prolonged luteal phases (Opsomer et al., 2000). In accordance with these studies, multiple logistic regression analysis demonstrated that the risk for abnormal milk 
progesterone profile increased with milk yield, GH (tendency), and parity (tendency), whereas it decreased with DMI (tendency) in this experiment.

It has been shown that ovarian follicular cyst formation frequently occurs in the early postpartum period when the cow changes from the acyclic state during pregnancy to the establishment of regular cyclicity (Webb et al., 1998; Vanholder et al., 2006). Low circulating IGF-I in early lactating cows could predispose to anovulation and development of cystic follicles (Zulu et al., 2000). Moreover, decreased circulating insulin and insulin resistance in early lactating cows may affect cyst formation (Vanholder et al., 2005) whereas leptin may play a role in cyst development as a minimum level of circulating leptin is required to induce the first postpartum LH surge (Vanholder et al., 2006). Furthermore, cows with cysts were associated with higher milk production (Garverick, 1997; Webb et al., 1998) and cows with high circulating NEFA after calving were twice as likely to develop cystic ovarian disease (Jackson et al., 2011). Additionally, Borromeo et al. (1998) demonstrated that GH concentrations in plasma and follicular fluid were higher in dairy cows with cystic follicles and Kawashima et al. (2007) showed that cows did not ovulate had higher circulating GH and lower circulating glucose during the peri-partum period than cows ovulated. In aggreement with the above mentioned studies, multiple logistic regression analysis demonstrated a negative effect on cystic body formation of circulating glucose and insulin, whereas circulating GH and NEFA had a positive effect on cystic body formation. Although all the animals expressed HIGH adiponectin levels were assessed without cystic bodies, there was no association between adiponectin level and follicular cysts. However, this needs to be investigated further as it is possibly biased by the small numbers of cows observed with follicular cysts (only 8 out of 60 ) and the small number of cows with HIGH adiponectin levels (6 out of 60) (Table 5.9). Moreover, follicular cyst formation delayed days to first oestrus in the present study (Figure 5.6) and may be another explaination why cows with HIGH adiponectin levels had fewer days to first oestrus than cows with NORMAL adiponectin levels (Table 5.7).

Interval from calving to conception was not affected by circulating adiponectin levels, whereas days to conception positively associated with days to first oestrus, and cows with 
HIGH adiponectin levels had fewer days to first oestrus (43 days versud 56 days) than cows with NORMAL adiponectin levels in the present study. According to Figure 5.8 animals with 43 days to first oestrus (HIGH adiponectin levels) did not have statistically different days to conception (74 days versus 80 days) compared with animals with 56 days to first oestrus (NORMAL adiponectin levels). Insulin was the main factor influenced days to conception, but circulating insulin was not different between cows with HIGH and NORMAL adiponectin levels. This latter may further explain why interval from calving to conception was unaffected by circulating adiponectin levels.

Why individual cows had consistently high or low adiponectin levels is difficult to explain in this experiment. In humans, circulating adiponectin has a strong genetic component with heritability estimated to be between $30 \%$ and 50\% (Comuzzie et al., 2001). Moreover, low circulating adiponectin is observed in several forms of diabetes, with insulin resistance, and in non-alcoholic fatty liver disease (Xu et al., 2007). In dairy cows, there has been no genetic association study to examine the role of adiponectin polymorphisms on indices of insulin sensitivity, circulating GH levels, fatty liver disease, and glucose tolerance. However, it is possible that insulin resistance, genetics, and fatty liver disease in dairy cows to regulate circulating adiponectin levels similarly to man. However, this hypothesis needs to be further investigated.

To conclude, cows with HIGH adiponectin levels had fewer days to first oestrus and a higher probability to be pregnant than cows with NORMAL adiponectin levels. All, cows with HIGH adiponectin levels exhibited normal milk progesterone profiles. However, there were no significant difference between cows with HIGH adiponectin levels and cows with NORMAL adiponectin levels for days to pregnancy or follicular cyst formation. Differences in metabolic and hormonal profile and production traits may at least in part explain the differences in reproductive performance between cows with HIGH adiponectin levels and cows with NORMAL adiponectin levels. However, further work is required to clarify the reasons for existence of such "special" animals (with increased adiponectin, decreased GH, and superior fertility), and the relations of high circulating adiponectin with metabolic and hormonal profile, productive traits, and reproduction. 


\section{5.2. Interrelationships of adiponectin with glucose, BCS, metabolic hormones, and metabolites in high and low yielding cows}

Path diagrams and structural equation modelling (SEM) analysis for all the 60 cows in this experiment proved that glucose balance in dairy cows was regulated by the negative influences of circulating adiponectin, BOHB, and glucagon, and the positive influence of circulating insulin on circulating glucose. It is known that high circulating adiponectin levels were associated with a lower risk of development of type 2 diabetes and adiponectin was found to negatively correlate with circulating glucose (Hotta et al., 2000; Weyer et al., 2001; Spranger et al., 2003) and this is in agreement with the current study. Also, the hypoglycaemic effect of insulin action is antagonized by glucagon, and insulin is positively correlated with glucose (Brockman, 1978, 1979). Moreover, BOHB is negatively correlated with insulin and glucose in dairy cows (Drackley, 1999; Bobe et al., 2004; Veerkamp et al., 2003; Leroy et al., 2010). These findings are also in line with the current study. In the present study, GH, NEFA, leptin, and BCS had no direct effect on circulating glucose, but GH was negatively correlated with adiponectin and leptin, whereas leptin was positively correlated with insulin. Human obese patients showed decreased circulating GH and increased circulating leptin (Casanueva \& Dieguez, 1998) whereas acromegalic and anorexia nervosa patients expressed decreased circulating leptin and increased circulating GH (Popovic et al., 2001; Scacchi et al., 1999). Also, studies in humans and rodents showed that the relation between adiponectin and $\mathrm{GH}$ is possibly negative (Lam et al., 2004; Nilsson et al., 2005; Rodriguez-Pacheco et al., 2007). Insulin was positively correlated with leptin in periparturient and lactating dairy cows (Block et al., 2001; Garnsworthy et al., 2008c). These findings are also in agreement with the present study.

Previous milk yield (PMY) was used in the present study as an indicator of genetic merit for milk yield. However, it is known that other factors, such as lactation persistency, BCS at calving, DMI, calving interval, parity, health status, and diet contribute to differences observed in milk yield (Rook \& Thomas, 1983). Cows were grouped according to milk yield in the previous lactation (PMY) with a cut-off point between high and low yielding cows of $10,000 \mathrm{~kg} / \mathrm{cow} /$ lactation. This cut-off point is higher than the current average yield 
per cow in the UK $(7,406 \mathrm{~kg} / \mathrm{cow} /$ lactation) (DEFRA, 2011) and it was chosen to be as high as possible. This was to ensure that the nature of the differences observed in the PMY groups was mainly due to genetic differences between cows. All instances of ill health of cows were recorded in this experiment, although subclinical diseases cannot be excluded from the factors affecting PMY. Parity was not statistically different for high PMY cows compared to low PMY cows (the majority of the cows (39 out of 60) were in their $2^{\text {nd }}$ lactation) and the length of lactation used to calculate PMY was 305 days. In addition, high PMY cows had higher milk yield, DMI, and BCS at calving and a trend for higher $\triangle \mathrm{BCS}$ than low PMY cows in the present lactation, which is in agreement with the profile of the high genetic merit cow for milk production (Veerkamp et al., 2003).

Structural equation modelling analysis for cows with LOW PMY revealed a simple network of interrelationships of adiponectin with glucose and other metabolic signals in these animals. In PMY LOW cows, glucose homeostasis was regulated by the positive effect of insulin and the negative effects of adiponectin and glucagon. GH was not related to leptin or adiponectin. It is known that low yielding cows have low circulating GH (Veerkamp et al., 2003), which is a possible reason for the lack of correlation between GH and adiponectin. In these PMY LOW cows, adiponectin was the main insulin sensitizing hormone due to its direct negative effect on circulating glucose, whereas leptin may have exerted insulin stimulatory effects because it was correlated with insulin.

In contrast, PMY HIGH cows showed completely different patterns in regulating glucose homeostasis. In these animals, the relationships between metabolic hormones and metabolites were very complicated. It is known that selection for milk yield resulted in decreased circulating glucose and increased circulating GH and BCS (Roche et al., 2009; Coffey et al., 2003; Veerkamp et al., 2003). Possibly, that is the reason GH showed strong negative correlations with adiponectin and leptin. It has been demonstrated that GH is upregulated in human diabetes type 2 (Holt, 2003); high yielding cows tend to be genetically and phenotypically thinner (Garnsworthy, 2006); and fatter cows at calving lose more body fat than thinner cows (Garnsworthy \& Topps, 1982). This latter might explain the strong negative correlations of BCS with glucose, glucagon and GH and the strong positive 
correlations of BCS with insulin and adiponectin. Furthermore, glucose homeostasis in PMY HIGH cows was regulated by the positive effect of insulin and the negative effects of BCS and BOHB. In these cows, adiponectin did not play any direct role in regulating glucose balance (because it was strongly down-regulated by GH) but leptin, as in PMY LOW cows, was the main insulin stimulatory hormone because it was positively correlated with insulin. However, adiponectin was positively correlated with BCS and BCS had a significant effect on glucose balance. That means that, the greater was the BCS of the high yielding cows postpartum (cows that did not lose a lot of condition), the lower was the circulating GH and glucagon, and consequently the lower was the milk yield. Moreover, the lower the circulating GH, the higher was the circulating adiponectin, leptin, and BOHB. The higher the circulating leptin, the higher was the circulating insulin, NEFA, and glucose. Finally, this aetiological chain concludes to a profile of high yielding cows with elevated circulating adiponectin, leptin, insulin, glucose, NEFA, and BOHB. According to Figure 5.14, adiponectin and leptin are not active in terms of lowering glucose, and consequently that aetiology leads to elevated circulating glucose, insulin, NEFA, and BOHB which is common in human metabolic syndrome, insulin resistance, and diabetes mellitus. Oligomerisation of adiponectin structure is considered very important for its biological function (Berg et al., 2002; Barb et al., 2007) and could explain at least theoretically why high circulating adiponectin was possibly inactive under such physiological conditions. Moreover, impaired oligomerization might be an important causative factor for type 2 diabetes (Xu et al., 2007). The positive correlations of NEFA with insulin and leptin also imply insulin or/and leptin resistance in PMY HIGH cows (Krentz, 1996; Fam \& Andrikopoulos, 2007; Myers et al., 2008). Indeed, many studies have reported low insulin sensitivity in early lactation cows (Sano et al., 1991, 1993; Chilliard et al., 1998; Gutierrez et al., 2006; Hayirli, 2006). Besides, if this aetiological chain runs for a high yielding cow which loses a lot of condition postpartum, then it would fit the profile of an animal with high GH, milk yield, and glucagon and low glucose, insulin, leptin, adiponectin, NEFA, and BOHB. Of course, this is an incorrect prediction of the model because at least NEFA and BOHB are reported to be elevated in such conditions (Jorritsma et al., 2003; Veerkamp et al., 2003). However, the majority of commercial cows are in-between the two main cases examined by the model, and other physiological 
regulatory factors are likely to contribute to the whole complicacy of glucose homeostasis in high yielding cows.

The main conclusion of this analysis is that the relationship between adiponectin and GH was generally antagonistic in dairy cows. Also, the relation between circulating glucose and circulating adiponectin was negative and that might indicate insulin sensitizing traits of adiponectin in dairy cows. Moreover, high yielding cows might regulate glucose homeostasis differently to low yielding cows, which was mainly achieved by higher secretion of GH and lower or inactive adiponectin. GH possibly down-regulated leptin and adiponectin, but this was mainly in high yielding cows. Leptin was positively correlated with insulin in both high yieldings and low yielding cows, and it was likely to exert insulin stimulatory effects. However, further work is needed to elucidate the perplexities of glucose homeostasis and its interrelationships with hormonal and metabolic profile in dairy cows.

\subsubsection{Conclusions}

In summary, the results from this study confirm that cows with circulating adiponectin levels up to three times normal had a better reproductive performance than cows with normal circulating adiponectin levels. In addition, differences in metabolic and hormonal profile and production traits may at least in part explain the superior fertility of cows with circulating adiponectin levels up to three times normal. More importantly, the relation between circulating glucose and circulating adiponectin was negative and that might indicate insulin sensitizing traits of adiponectin in dairy cows. Finally, high yielding cows might regulate glucose homeostasis differently to low yielding cows due to increased GH and decreased adiponectin concentrations. However, these results need to be investigated further 


\section{General discussion}

\subsection{INTRODUCTION}

The control of glucose homeostasis and homeorhesis is of outmost importance in understanding hormonal and metabolic profiles, relating to NEB in early lactation (Bell, 1995; Bauman, 2000). The postpartum cow has to deal with energy deficit and the great demand for nutrients, especially glucose, to meet the high needs of the mammary gland (Butler \& Smith, 1989; Bell, 1995; Nebel \& McGilliard, 1998). Decreased insulin allows cows to partition glucose preferentially to the mammary gland, and increased circulating GH and prolactin facilitate this adaptation (Bauman \& Currie, 1980). Elevated circulating NEFA, BOHB, and urea are mainly produced as a result of excessive mobilization of adipose and muscle tissue to support the demand for nutrients by the mammary gland (Bell, 1995; Grummer, 1995). Decreased circulating IGF-I may be a consequence of deteriorating functionality of the liver which in early lactation prioritizes intensification of processes such as gluconeogenesis, lipolysis, and ketogenesis (Butler et al., 2003; Wathes et al., 2007, 2008). Adiponectin, because of its insulin sensitising actions and its negative correlation with body condition (Berg et al., 2002; Matsuzawa et al., 2004; Kadowaki \& Yamauchi, 2005), could be another putative regulator of metabolism during the transition from pregnancy to lactation (Mazaki-Tovi et al., 2005; Mitchell et al., 2005). Body condition score is considered an indirect measure of nutritional status (Short et al., 1990; Garnsworthy, 2006; Bewley \& Schutz, 2008) and nutrition has been demonstrated to interact with reproduction (Boland et al., 2001; Garnsworthy et al., 2009).

The overall objective of the present study was to examine the impacts of different nutritional and body condition treatments on metabolic and hormonal profiles, circulating adipokines, and reproductive performance in dairy cows. A special focus was directed to circulating adiponectin and its association with metabolic and hormonal status and reproduction. Work in this thesis has shown some novel effects of circulating adiponectin on cow fertility. In addition, the effects of lactational stage, diet, and BCS at calving on 
circulating adiponectin were assessed. Also, adiponectin was shown to be present in bovine milk for the first time. Moreover, optimum insulin concentration in terms of reproduction, and its association with dietary and condition treatments was explored. Furthermore, a subpopulation of dairy cows with consistently high circulating adiponectin levels was identified for the first time, and the hormonal, metabolic, reproductive, and productive profiles of these animals were studied. Last but not least, the association of adiponectin with glucose homeostasis and other metabolic and hormonal signals was determined in cows with different milk yield potential. To our knowledge, no other study has explored associations between circulating adiponectin and hormonal and metabolic stimulus with regard to milk yield potential in dairy cows.

\subsection{SUMMARY OF MAIN POINTS}

\subsubsection{Effect of diet and body condition score at calving on reproductive performance in dairy cows}

Results from the present study demonstrated that feeding dairy cows with a HS diet or a HF diet for the first 4 months of lactation would not result in changing reproductive performance (probability of cows to be pregnant, days to first oestrus, and days to conception, were not affected by dietary treatment). That is mainly because important metabolic hormones (e.g. leptin, IGF-I, and GH), production traits (milk yield, BCS, $\triangle \mathrm{BCS}$, nadir week, and nadir BCS), and metabolites (e.g. NEFA and glucose) were not influenced by diet (Chapter 3). In addition, moderately elevated insulin concentration that exceeded optimum insulin concentration and induced by feeding dairy cows the HS diet may account for the higher probability to express ABNORMAL milk progesterone profile (Chapter 3). The results of the present study are not in agreement with the study of Gong et al (2002b) in which cows fed the HS diet had better reproductive performance than cows fed the HF diet. An explanation for the discrepancies between the present study and the study of Gong et al (2002b) could be that the animals were dietary manipulated for longer (up to 2 times) in the present study than the animals of the study of Gong et al. (2002b). Moreover, all animals exceeded minimum insulin concentration in terms of reproduction in the present study whereas some animals were below minimum insulin concentrations in the 
study of Gong et al. (2002b) and that might be another explanation for discrepancies between the two studies (Chapter 3).

The results of the present study clearly indicated that THIN cows at calving (BCS $\leq 3.25$ ), which lost less than 0.5 units of BCS postpartum had better reproductive performance than FAT cows at calving (BCS $>3.25$ ), which lost more than 0.8 units of BCS postpartum (Chapter 3). FAT cows at calving had higher circulating NEFA and lower circulating IGF-I than THIN cows at calving. Elevated circulating NEFA may detrimentally affect follicular growth and development (Leroy et al., 2008, 2010) and reduced circulating IGF-I negatively influence postpartum reproductive performance (Butler et al., 2000; Huszenicza et al., 2001; Meikle et al., 2004). Moreover, THIN cows at calving had higher probability of being pregnant and lower probability of expressing irregular oestrous cycles than FAT cows at calving, although the animals had the same insulin concentration, which ranged from 0.4 to $0.6 \mathrm{ng} / \mathrm{ml}$. This latter result could also explain why THIN cows showed superior reproductive performance compared with FAT cows in the present study (Chapter 3).

\subsubsection{Reproductive performance and optimum insulin concentration}

Long term moderately elevated insulin concentration $(>0.3 \mathrm{ng} / \mathrm{ml})$ had a negative impact on pregnancy rate and milk progesterone profile in the present study, whereas optimum insulin concentration that maximized pregnancy rate and minimized the probability for cows to express abnormal milk progesterone profile varied from 0.2 to $0.3 \mathrm{ng} / \mathrm{ml}$. Furthermore, insulin concentration greater than $0.6 \mathrm{ng} / \mathrm{ml}$ reduced the probability of cows being pregnant towards zero, whereas insulin greater than 0.4 to $0.6 \mathrm{ng} / \mathrm{ml}$ tended to maximize the probability for cows to express an abnormal milk progesterone profile (Chapter 3). Also, insulin concentration greater than $0.7 \mathrm{ng} / \mathrm{ml}$ resulted in calving to conception interval greater than 100 days, whereas animals with insulin concentration within the range $0.2-0.3 \mathrm{ng} / \mathrm{ml}$ had a calving to conception interval of less than 70 days (Chapter 5). Therefore, the results suggest that optimum insulin concentration in terms of reproduction is within the range 0.2-0.3 $\mathrm{ng} / \mathrm{ml}$, whereas insulin concentration greater than 
0.6 to $0.7 \mathrm{ng} / \mathrm{ml}$ is detrimental for cow fertility. Interestingly, the current study found that BCS at calving was a critical modulator of fertility in lactating dairy cows when circulating insulin was suboptimum $(\geq 0.4 \mathrm{ng} / \mathrm{ml})$. Moreover, THIN cows at calving had higher probability to be pregnant and lower probability to express abnormal milk progesterone profile than FAT cows at calving, when the animals had approximately the same insulin concentration and insulin concentration ranged from 0.4 to $0.6 \mathrm{ng} / \mathrm{ml}$. Thus, achieving a BCS of less than 3.25 units at calving is essential to ensure optimum cow fertility. Furthermore, cow fertility (pregnancy rate and milk progesterone profile) was not affected by dietary treatments when insulin concentrations were about the same and insulin ranged from 0.1 to $1 \mathrm{ng} / \mathrm{ml}$. However, the current work showed that HF diet must be the preferable feeding strategy to maximise reproductive performance of dairy cows when insulin concentration ranges from 0.1 to $0.3 \mathrm{ng} / \mathrm{ml}$ and BCS at calving is equal or lower than 3.25 units.

\subsubsection{Adiponectin}

\subsubsection{Measurement of circulating adiponectin in dairy cows}

The present study showed that plasma adiponectin in dairy cows varied from $6.47 \pm 0.75$ to $11.15 \pm 1.06 \mathrm{ng} / \mathrm{ml}$, and some cows had constantly elevated plasma adiponectin throughout the experiment. In addition, circulating adiponectin values were not normally distributed and mean circulating adiponectin was significantly greater than mean insulin, leptin, GH and glucagon. Furthermore, the high autocorrelation of circulating adiponectin values might indicate that plasma adiponectin concentration was tightly controlled genetically and other factors had only a minimal impact (Chapter 4).

\subsubsection{Measurement of adiponectin in bovine milk}

Milk adiponectin concentration was measured for the first time in bovine milk and the mean concentration was $11.5 \pm 2.6 \mathrm{ng} / \mathrm{ml}$ (Chapter 4). This result is in agreement with the studies of Martin et al. (2006), Weyermann et al. (2006), and Bronsky et al. (2006) which confirmed the presence of adiponectin in human milk. Milk and plasma adiponectin 
concentrations were similar in the current study, which may imply that milk adiponectin is excreted at concentrations similar to the peripheral circulation (Chapter 4). Adiponectin concentrations in human breast milk ranged from 3.9 to $87.9 \mathrm{ng} / \mathrm{ml}$ (Bronsky et al., 2006; Martin et al., 2006). Normal adiponectin concentrations in human serum ranged from 5 to $30 \mu \mathrm{g} / \mathrm{ml}$ (Ahima, 2006) and this may imply adiponectin is present in human milk in concentration lower than in blood, which is not in agreement with the present study. Ohtani et al. (2011) demonstrated the existence of an autocrine-paracrine system of adiponectin action in bovine mammary gland and the presence of adiponectin receptors was shown in human breast cancer cell line MCF-7 (Dieudonne et al., 2006). However, there is no study that directly compared circulating and milk adiponectin or examined the action, synthesis, and excretion of adiponectin in the mammary gland. Human milk adiponectin concentrations decrease approximately 5\% - 6\% with each month of lactation (Newburg et al., 2010; Savino \& Liguori, 2008). In the present study, milk adiponectin concentrations did not show the same trend, although samples were collected only for the first two months of lactation; nevertheless, adiponectin varied with days postpartum. Another finding of this study was that milk adiponectin was not different for cows with either high or low milk yield, but this needs to be confirmed (Chapter 4).

The milk adiponectin assay, because it is non-invasive, offers the potential for more widespread studies in dairy cows under commercial conditions. It was intended to apply the technique on a larger scale in the present study, but time and financial constraints precluded this. The effects of adiponectin and adiponectin receptors in mammary gland and milk synthesis need to be elucidated by explicit experiments.

\subsubsection{The impact of lactational stage, diet and BCS at calving on circulating adiponectin in dairy cows}

This study showed that, as in humans (Asai-Sato et al., 2006; Ritterath et al., 2008), the transition from pregnancy to lactation in dairy cows was associated with a reduction in plasma concentration of adiponectin. Moreover, the present study found that after the $4^{\text {th }}$ week postpartum circulating adiponectin was significantly increased and remained at the same level until the $12^{\text {th }}$ week postpartum. Furthermore, this study suggests that after the 
$12^{\text {th }}$ week postpartum there was a significant reduction in circulating adiponectin (Chapter 4).

In the present study, stage of lactation affected the majority of circulating metabolic hormones, metabolites and production traits, but circulating adiponectin showed correlations only with circulating $\mathrm{GH}$ and postpartum BCS loss $(\triangle \mathrm{BCS})$. However, there was a weak negative association between plasma adiponectin and glucose. The weekly changing pattern of circulating adiponectin can be partially explained by the negative relationship between GH and adiponectin. This is the first in vivo study to demonstrate a negative relationship between GH and adiponectin in dairy cows (Chapter 4).

There was no effect of either diet or BCS at calving on circulating adiponectin. Circulating GH and glucose were not altered by dietary and BCS treatments in this study and this was possibly the reason the diet and BCS at parturition, had no effect on circulating adiponectin concentrations (Chapter 4). However, dietary factors have modulated circulating adiponectin (Reis et al., 2010) in other species. High consumption of magnesium (Qi et al., 2005; Cassidy et al., 2009), caffeine (Williams et al., 2008), n-3 PUFA (Duda et al., 2007), and dietary salt (Lely et al., 2007) are associated in humans with higher circulating adiponectin. Prepartum thiazolidinediones (TZD) administration dramatically decreased $\triangle \mathrm{BCS}$ and NEFA and increased DMI and the proportion of dairy cows ovulating by 21 days postpartum (Schoenberg et al., 2008; Smith \& Overton, 2008). TZD is a known PPAR $\gamma$ agonist, which increases adiponectin production in both humans and rodents (Xu et al., 2007; Yoon et al., 2009). It is likely that the therapeutic effects of TZD are mediated by induction of adiponectin (Xu et al., 2007; Yoon et al., 2009). TZD administration of cows requires drug approval; however, application of dietary strategies enhancing circulating adiponectin may be an inexpensive alternative. Thus, properly designed experiments need to be conducted in order to evaluate putative roles of nutrients in regulating circulating adiponectin in dairy cows

A characteristic trait of circulating adiponectin is its negative correlation with adiposity in non-pregnant human and rodent models (Ahima, 2006). However, circulating adiponectin values throughout pregnancy are not correlated with maternal BMI in humans (Mazaki- 
Tovi et al., 2005; Jansson et al., 2008). According to Raddatz et al., (2008) and Puntenney (2006) adiponectin was not correlated with BCS in periparturient cows and this finding is in accordance with the current study. Thus, it is likely that BCS is not correlated with circulating adiponectin in periparturient and early lactating dairy cows. However, further research would determine if this hypothesis is correct.

Postpartum BCS loss $(\triangle \mathrm{BCS})$ was negatively correlated with leptin and adiponectin in the current study and may be a useful tool to study circulating adipokines in periparturient and early lactating cows (Chapter 4). The negative correlation of leptin with BCS loss is documented in heifers (Leon et al., 2004) and this is in agreement with the finding of the present study. Weight and BMI loss lead to increased circulating adiponectin in humans (Reinehr et al., 2004; Bobbert et al., 2005; Engl et al., 2007), but this is not in line with the present study. A possible explanation for this discrepancy between the present study and human studies could be differences between human and bovine animal models in regulation of circulating adiponectin. Also, it is known that human adiponectin is downregulated when hyperinsulinemia and insulin resistance coexist (Kadowaki \& Yamauchi, 2005). The animals of the present study had moderately elevated insulin concentrations and that might be another reason why $\triangle \mathrm{BCS}$ was negatively correlated with adiponectin. In humans, circulating adiponectin showed a strong negative relationship with visceral body fat, which is strongly related to BMI (Matsubara et al., 2002; Cnop et al., 2003; LaraCastro et al., 2006). It is uncertain if $\triangle \mathrm{BCS}$ is related to visceral body fat of the cow.

\subsubsection{Circulating adiponectin and milk yield}

Evidence accumulated from in vitro studies suggests that human mammary epithelial cells of various cancer cell lines express receptors of adiponectin (Dieudonne et al., 2006; Treeck et al., 2008; Jarde et al., 2009). Treatment of cancer mammary epithelial cells with recombinant adiponectin deters their growth, by activating cell apoptosis pathways and by inhibiting the cell cycle (Dieudonne et al., 2006). Moreover, obesity causes suppression of circulating adiponectin levels (Kadowaki \& Yamauchi, 2005) and genetic improvement of cows for higher milk yield results in increased postpartum BCS loss (Dechow et al., 2002) and circulating GH (Veerkamp et al., 2003). Furthermore, circulating GH was negatively 
correlated with circulating adiponectin in dairy cows (as shown in Chapter 4) and adiponectin receptors were present in bovine mammary epithelial cells (Ohtani et al., 2011). It is likely; therefore, to assume low circulating adiponectin (as a result of elevated GH concentrations) leads to increased milk production in high yielding cows. High yielding cows lost more condition postpartum, ate more feed and had higher circulating GH, milk yield and DMI but lower circulating adiponectin, leptin, and insulin than low yielding cows in the current study (Chapter 4). Moreover, cows with circulating adiponectin levels up to three times normal $(21.2 \pm 1.32 \mathrm{ng} / \mathrm{ml})$ had lower GH and milk yield and higher leptin (tendency) than cows with normal circulating adiponectin levels $(7.1 \pm 0.23 \mathrm{ng} / \mathrm{ml})$ as shown in Chapter 5. This is the first study to suggest that due to increased circulating GH and its antagonistic relationship with adiponectin and leptin, high yielding cows may have lower circulating leptin and adiponectin concentrations than low yielding cows.

\subsubsection{Adiponectin and feed intake in dairy cows}

This is the first study to show that adiponectin is likely to be related to DMI in dairy cows, but this relationship is minimal and dependent on BCS at calving (Chapter 4). Kubota et al., (2007) showed that adiponectin enhances AMPK activity in the murine arcuate hypothalamus through AdipoR1 receptor, and stimulates food intake. There is a need for further study of the effect of adiponectin on food intake.

\subsubsection{Effect of circulating adiponectin levels on reproductive performance}

For the first time, a subpopulation of dairy cows with consistently high circulating adiponectin levels was identified, and the hormonal, metabolic, reproductive, and productive profiles of these animals were studied. Cows with circulating adiponectin levels up to three times normal $(21.2 \pm 1.32 \mathrm{ng} / \mathrm{ml})$ had a higher probability to be pregnant and fewer days to first oestrus than cows with normal circulating adiponectin levels $(7.1 \pm 0.23$ $n g / m l)$. Also, cows with circulating adiponectin levels up to three times normal had a tendency to be associated with normal milk progesterone profile than cows with normal circulating adiponectin levels. However, there were no significant difference between cows 
with circulating adiponectin levels up to three times normal and cows with normal adiponectin levels in the interval from calving to conception and formation of follicular cysts. Differences in metabolic and hormonal profiles and reproductive and production traits may at least partly explain the differences in reproductive performance between cows with high circulating adiponectin and cows with normal circulating adiponectin levels (Chapter 5).

Unfortunately, only $10 \%$ of cows in this experiment expressed circulating adiponectin level up to three times normal. In humans, circulating adiponectin has a strong genetic component with heritability to be estimated between $30 \%$ and $50 \%$ (Comuzzie et al., 2001). Moreover, the high autocorrelation of circulating adiponectin values in the present study may indicate that plasma adiponectin concentration is tightly regulated genetically. Genetic association studies need to be performed in dairy cows to investigate further if there is any association between adiponectin gene polymorphisms and indices of insulin sensitivity, circulating GH levels, circulating adiponectin levels, and glucose resistance.

\subsubsection{Glucose homeostasis}

Glucose balance in dairy cows was regulated mainly by the negative influence of circulating adiponectin, BOHB, and glucagon on circulating glucose, and the positive influence of circulating insulin on circulating glucose (Chapter 5).

High yielding cows might regulate glucose homeostasis differently to low yielding cows, which was mainly achieved by higher secretion of GH and lower or inactive adiponectin. GH possibly down-regulated leptin and adiponectin, but this was mainly in high yielding cows. Leptin was positively correlated with insulin in both high yielding and low yielding cows, and it was likely to exert insulin stimulatory effects (Chapter 5). 


\subsubsection{Reproductive performance and adipokines}

Reproductive indices (such as pregnancy rate, days to first oestrus, interval from calving to conception, milk progesterone profile, and follicular cyst formation) were measured and the effects of circulating adiponectin and leptin were examined. Leptin was negatively associated with days to first oestrus in the present study (Chapter 5), and this is in agreement with the studies of Liefers et al. (2003), Kadokawa et al. (2000), Block et al. (2001), and Meikle et al. (2004) in dairy cows.

Circulating adiponectin was negatively associated with the probability of cows to express abnormal milk progesterone profiles and cows with circulating adiponectin levels up to three times normal had a tendency to be associated with normal milk progesterone profiles (Chapter 5). According to Campos et al. (2008) there is plenty of evidence to support a beneficial role of adiponectin in reproduction, but it is not yet clear that adiponectin is required for normal reproductive performance. This is the first study to suggest that hypoadiponectineamia might be associated with increased milk production and a predominance of atypical milk progesterone profiles. This could be another mechanism that leads to infertility in the modern dairy cow; however, explicit experimentation is needed to further investigate this hypothesis.

\subsection{INTEGRATION OF RESULTS AND IMPACT ON REPRODUCTIVE MANAGEMENT}

Cow fertility was the final output of complicated interactions of BCS at calving, diet, metabolic and hormonal stimulus, production traits, and reproductive indices in the current project. An overview of factors influenced reproductive indices and subsequent cow fertility is presented in Figure 6.1.

Results in this thesis have demonstrated the predominant role of insulin in cow fertility. Pregnancy rate, interval from calving to conception, and milk progesterone profile were associated with insulin and analysis showed that optimum insulin concentration that benefited these indices was within the range 0.2 to $0.3 \mathrm{ng} / \mathrm{ml}$, whereas insulin concentration greater than 0.6 to $0.7 \mathrm{ng} / \mathrm{ml}$ deteriorated them. However, analysis in Chapter 
5 showed that insulin concentration greater than 0.6 to $0.7 \mathrm{ng} / \mathrm{ml}$ could benefit cow fertility due to decreased incidence of follicular cyst formation. Thus, insulin effects on fertility can vary significantly according to insulin concentrations and reproductive indices assessed. Follicular cyst formation positively associated with days to first oestrus and HS diet increased circulating insulin in this study. This latter may suggest that HS diets can be used to benefit fertility in dairy herds with history of low insulin concentration, increased incident of follicular cyst formation, and delayed onset of postpartum ovarian activity. However, it needs to be further elucidated.

Proportion of atypical oestrous cycles was low and pregnancy rate was high when insulin concentration was within the optimum range in the current project. Also, the pregnancy rate was higher when the proportion of atypical oestrous cycles was lower. Therefore, cow fertility was maximized when insulin concentration was within the optimum range. Moreover, optimum calving interval is 12 months in dairy cows (Ball \& Peters, 2004) and maintenance of insulin concentration within the optimum range can assure on time reproduction of commercial dairy herds, which is important for their profitability. Consequently, results of this study support the concept that cow fertility will be enhanced if insulin concentration is within the range 0.2 to $0.3 \mathrm{ng} / \mathrm{ml}$ for a period of 4 months postpartum. This significant finding should be taken in account when a postpartum dietary strategy is designed to improve reproductive performance of dairy cows. Of the managerial tools studied in the current project, HS and HF diets showed a potential to regulate insulin concentrations and should be components of an integrated management strategy that ensures better cow fertility. However, long term use of HS diet should be avoided because it increases the proportion of irregular oestrous cycles.

Results of this study support the concept that $\triangle \mathrm{BCS}$ and BCS at calving are critical modulators of fertility in lactating dairy cows. Optimum range of $\triangle \mathrm{BCS}$ in terms of reproduction was $0.25-0.50$ units of $\mathrm{BCS}$, whereas $\triangle \mathrm{BCS}$ greater than $0.70-0.80$ units of BCS was detrimental for cow fertility. Moreover, THIN cows at calving $(\mathrm{BCS} \leq 3.25)$ had better reproductive performance than FAT cows at calving $(\mathrm{BCS}>3.25)$. Furthermore, the results demonstrated that optimum insulin concentration was dependent on BCS at calving in lactating dairy cows and THIN cows at calving $(\mathrm{BCS} \leq 3.25)$ had better reproductive 
performance than FAT cows at calving $(\mathrm{BCS}>3.25)$ when insulin concentration was within the range 0.4-0.6 $\mathrm{ng} / \mathrm{ml}$. Also, THIN cows at calving had lower $\triangle \mathrm{BCS}$ and circulating NEFA, and higher circulating IGF-I than FAT cows at calving. NEFA was positively associated with follicular cyst formation and IGF-I was positively associated with pregnancy rate, and negatively with interval from calving to conception in the current study. In addition, $\triangle \mathrm{BCS}$ was positively associated with days to first oestrus and negatively with pregnancy rate. Therefore, achieving a BCS of less than 3.25 units at calving is essential to ensure optimum cow fertility. The results also suggest that HF diet must be the preferable feeding strategy to maximize reproductive performance of lactating dairy cows when insulin concentration ranges from 0.1 to $0.3 \mathrm{ng} / \mathrm{ml}$ and BCS at calving status is THIN.

The main effects of adipokines on reproduction were: adiponectin negatively influenced the proportion of animals expressed irregular oestrous cycles, whereas leptin negatively influenced days to first oestrus. $\triangle \mathrm{BCS}$ was negatively correlated with leptin and adiponectin and may be a useful tool to study circulating adipokines in periparturient and early lactating dairy cows. Moreover, animals with HIGH adiponectin levels were thin at calving $(\triangle \mathrm{BCS}=0.25, \mathrm{BCS}$ at calving $=3.13)$ whereas animals with NORMAL adiponectin levels were fat at calving $(\triangle \mathrm{BCS}=0.70, \mathrm{BCS}$ at calving $=3.45)$. This latter may further explain why animals with HIGH adiponectin levels showed superior reproductive performance.

In summary, the results from this study suggest that an integrated management strategy that maximizes cow fertility should be based on the following principles:

(1) Optimum insulin range $(0.2-0.3 \mathrm{ng} / \mathrm{ml})$ must be maintained for a period of 4 months postpartum.

(2) BCS at calving of less or equal than 3.25 units must be achieved

(3) $\triangle \mathrm{BCS}$ optimum range (0.25-0.50 units of BCS) must not be exceeded for a period of 4 months postpartum.

(4) Selected prepartum and postpartum feeding strategy must meet the above-mentioned goals. 


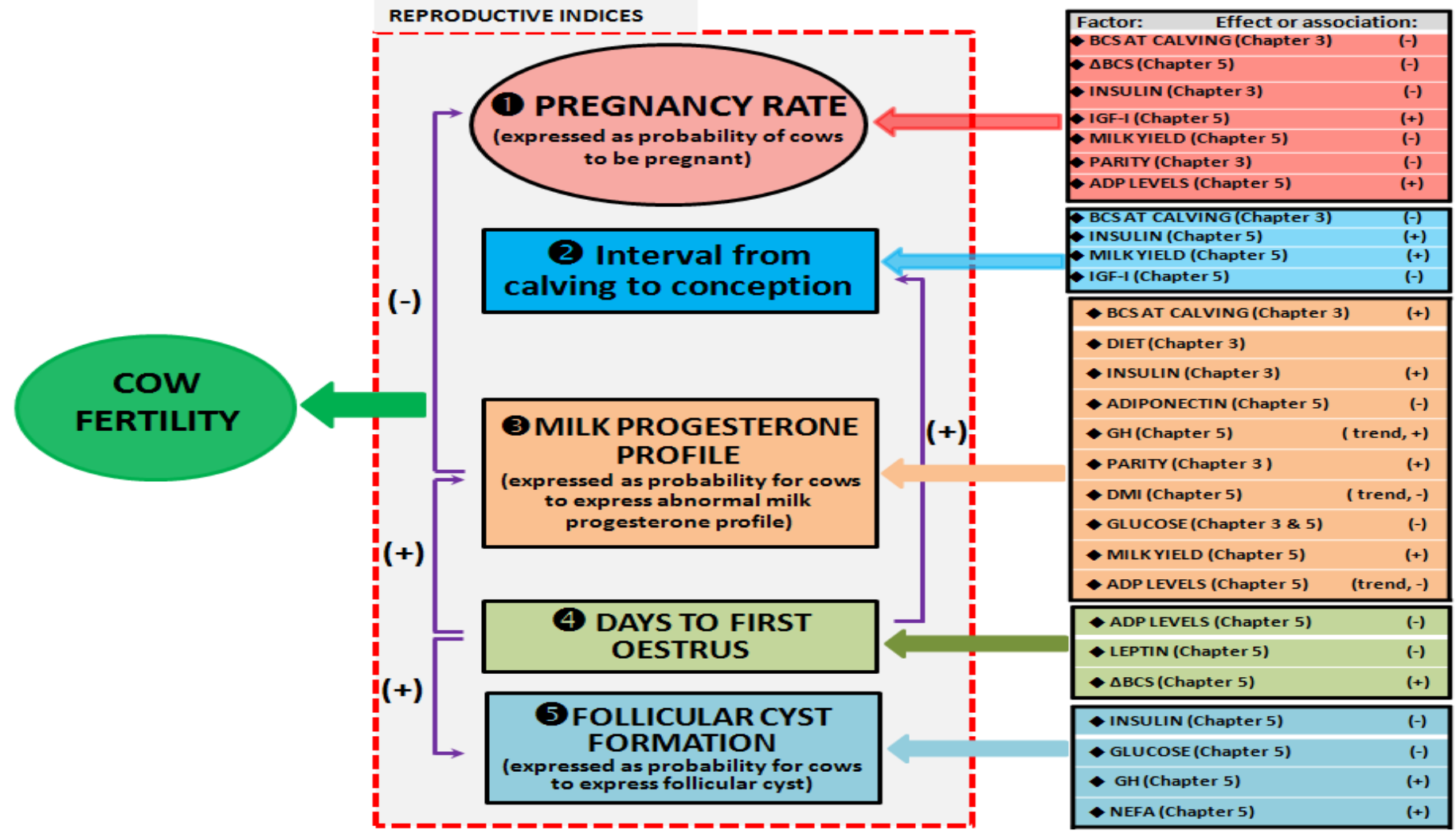

Figure 6.1: Overview of factors influenced reproductive indices and subsequent cow fertility in the present project. (D Pregnancy rate (expressed as probability for cows to be pregnant) was affected mainly by BCS at calving (Chapter 3), postpartum BCS loss $(\triangle \mathrm{BCS})(\mathrm{Chapter} 5)$, circulating insulin (Chapter 3), circulating IGF-I (Chapter 5), milk yield (Chapter 5), parity (Chapter 3), and milk progesterone profile (Chapter 3 \& 5). Also, circulating adiponectin levels had an effect on pregnancy rate (Chapter 5), whereas pregnancy rate was not influenced by dietary treatments (Chapter 3). 2Interval from calving to conception was influenced by BCS at calving (Chapter 3), circulating insulin (Chapter 5), circulating IGF-I (Chapter 5), and milk yield (Chapter 5); it was unaffected by the dietary treatments (Chapter 3) and circulating adiponectin levels (Chapter 5). Also, days to conception was influenced by days to first oestrus (Chapter 5). BMilk progesterone profile (expressed as probability for cows to express abnormal milk progesterone profile) was affected mainly by the dietary and BCS at calving treatments (Chapter 3), circulating insulin (Chapter 3), days to oestrus (Chapter 5), circulating adiponectin (Chapter 5), glucose (Chapter 3), parity (Chapter 3), and milk yield (Chapter 5). Also, cows with circulating adiponectin levels up to three times normal had a tendency to be nonrandomly associated with normal milk progesterone profile (Chapter 5). Moreover, milk progesterone profile showed a trend to be influenced by circulating GH (Chapter 5) and feed intake (Chapter 5). 4 Days to first oestrus was affected mainly by $\triangle \mathrm{BCS}$ (Chapter 5) and leptin (Chapter 5). Also, circulating adiponectin levels had an effect on days to first oestrus (Chapter 5), whereas days to first oestrus was not influenced by the dietary and BCS at calving treatments (Chapter 3). GFollicular cyst formation was affected mainly by circulating insulin, glucose, GH, and NEFA (Chapter 5). Also, follicular cyst formation positively associated with days to first oestrus. Positive sign $(+)$ indicates positive effect or association, whereas negative sign (-) indicates negative effect or association. 


\subsection{LIMITATIONS OF THE STUDY}

There are certain limitations in the present study that need to be acknowledged. The first limitation concerns the experimental design, which was repeated measurements (Davis, 2002). The data obtained from this study were panel or longitudinal data. Cows were the panels and treatments were nested within week of experiment. Repeated measurement design, was seen as the most appropriate design for the present study because it allowed definition of individual patterns of adiponectin values (Chapter 5). In contrast, crosssectional designs would require significantly more cows at different stages of lactation to achieve the same statistical power, without allowing assessment of individual patterns (Minkle, 1997; Davis, 2002; Liu \& Li, 2005).

Missing or incomplete data are inherent in studies where repeated measurements are obtained from animals (Little \& Rubin, 1987; Allison, 2001; Frees, 2004). However, missing data in panel studies can be handled by maximum likelihood $(M L)$, restricted maximum likelihood (REML), and multiple imputation estimation (MI) (Allison, 2001; Schafer, 1997; Frees 2004; Enders, 2010) methods. Generalized linear models (GLM), generalized estimating equations (GEE), and log-linear Poisson models utilize $M L$ to calculate parameter estimates and were used in the present study (McCullagh \& Nelder, 1989; Lindsey, 1997; Dobson, 2002). GEE and GLM do not assume normality, but allow choice of error distribution and link function, leading to more efficient parameter estimates (McCullagh \& Nelder, 1989; Lindsey, 1997).

The second limitation concerns measurement of plasma and milk adiponectin. There was no species specific validated RIA kit for measuring bovine adiponectin. Serial dilution of bovine plasma and fat-free milk led to samples contained adiponectin concentrations below the sensitivity of the assay. Thus, validation of the method was not feasible. However, the Linco kit has been used for cows (Raddatz et al., 2008), horses (Gordon \& McKeever, 2005; Gordon et al., 2006; Kearns et. al., 2006; Pratt et al., 2005), and dogs (Gayet et al., 2007; Brunson et al., 2007). Adiponectin was assessed in bovine milk by using the same kit, which is an extra reason to believe that kit can at least in part measure bovine adiponectin. Circulating adiponectin, as measured by the Linco kit, revealed some of its 
basic heterologous traits (Chapter 4), but development of a species specific bovine kit to measure circulating adiponectin might offer an opportunity to estimate plasma adiponectin more precisely in cattle. Analysis of circulating adiponectin levels in Chapter 5 was retrospective and few animals expressed HIGH adiponectin levels. Retrospective analysis is susceptible to bias error sources, but when the examined trait is rare or takes a long time to develop this design is the best choice in practice (Woodward, 1999).

Parameter estimation in structural equation models uses maximum likelihood $(M L)$, which is based on certain assumptions (i.e. large sample and multivariate normality). It is known that combining small sample sizes, non-normal data, and weak empirical relationships between variables can lead to estimation problems and unreliable results (Werner \& Schermelleh-Engel, 2009). It is questionable if the findings of the present study (Chapter 5) can be generalized beyond the cases studied. However, the research findings of the present study concerning glucose homeostasis should be tested in larger numbers of animals, to refine the models suggested.

As discussed above, another limitation of the analysis in the present study was that reproductive performance (Chapter 3 \& 5) was assessed in a small sample of cows. Moreover, reproductive hormones were not measured in the present study. Milk progesterone profile was transformed to a binominal variable with two levels (NORMAL and ABNORMAL). This was the best approach in practice because only a small minority of animals expressed DOV1, DOV2, PCL1, and PCL2, although the components of ABNORMAL milk progesterone patterns are not exactly the same in terms of reproduction (Lamming \& Darwash, 1998; Taylor et al., 2003).

Another consideration is that BCS at calving was generally high in both BCS groups (Chapter 3, 4, \& 5), so the groups consisted of fat versus over-fat cows. Because some hormonal and metabolic traits (e.g. adiponectin and leptin) are affected by adiposity, and the differences between the BCS groups were small, they possibly did not allow significant responses to be investigated fully. 


\subsection{RECOMMENDATIONS FOR FURTHER RESEARCH}

The research conducted in this project has led to some novel results and conclusions. The purpose of this section is therefore to identify and discuss the need for further research. The areas of further research include the following:

There is a critical need for additional comprehensive studies on the potential for reproductive performance in dairy cows to be modulated by BCS at calving and insulin. It is important to determine the best BCS at calving and optimum insulin concentration range in terms of reproduction, and what are the best management approaches for obtaining an optimum $\triangle \mathrm{BCS}$ and insulin range. Moreover, experiments need to be conducted to give a better understanding of the importance and role of insulin and $\triangle \mathrm{BCS}$ in fertility and productivity of dairy cows, and their association with hormonal and metabolic profiles.

It is known that genetic selection for milk yield led to higher circulating $\mathrm{GH}$ in dairy cows (Veerkamp et al., 2003) and insulin resistance is commonly observed in early lactating cows (Sano et al., 1991, 1993). This makes the early lactating dairy cow the most suitable animal model to study associations of adiponectin with GH and glucose. In the present study, the relationship between adiponectin and $\mathrm{GH}$ was antagonistic and the relation between circulating glucose and adiponectin was also negative. However, additional research is needed to confirm this negative association and the causes.

The current prevalence of obesity in humans in the UK is around $20 \%$, but rates are increasing rapidly (Norman, 2010). Obesity is related to diabetes mellitus and both are associated with metabolic complications such as hypoadiponectineamia, and subfertility (Ramsay et al., 2006). Increased risk of breast cancer is also associated with reduced circulating adiponectin (Mantzoros et al., 2004). Bovine adiponectin protein sequence shares about $85 \%$ homology with human adiponectin protein sequences (Berg et al., 2002). Interestingly, the presence of the insulin-sensitizing adipokine adiponectin in fat-free cow milk was ascertained by the present study. According to $\mathrm{Xu}$ et al. (2007) direct supplementation of recombinant adiponectin in human subjects is extremely expensive. Thus, consumption of fat-free cow milk (or dairy products) could be an alternative 
inexpensive source of adiponectin for humans, but this needs to be elucidated further by interdisciplinary research. Furthermore, $10 \%$ of the animals in the present study expressed circulating adiponectin levels up to three times normal, and milk and plasma adiponectin concentrations were similar, which may imply that milk adiponectin is excreted at concentrations similar to those found in blood. If that is the case, then selection of animals with circulating adiponectin levels up to three times normal would allow production of distinctive label milk with high adiponectin. However, explicit experimentation is needed to investigate if this hypothesis is true.

\subsection{OVERALL CONCLUSIONS}

This thesis has confirmed previous reports indicated that BCS at calving is a critical regulator of fertility in lactating dairy cows. THIN cows at calving (BCS $\leq 3.25)$, which lost less than 0.5 units of BCS ( $\triangle \mathrm{BCS}$ ) during the first 4 months of lactation, had superior reproductive performance, and that was because of increased circulating IGF-I and decreased circulating NEFA. Moreover, this study clearly showed that there was an optimum insulin concentration $(0.2$ to $0.3 \mathrm{ng} / \mathrm{ml})$ necessary for normal reproductive performance, while insulin concentration greater than $0.6 \mathrm{ng} / \mathrm{ml}$ impaired reproductive performance in lactating dairy cows. The results strongly suggested that optimal reproductive performance of dairy cow was dependent on insulin concentration and BCS at calving.

Adiponectin showed a potential to regulate glucose homeostasis and feed intake in the current study, and it was detectable in bovine milk. Also, genetic selection for milk production due to increased circulating $\mathrm{GH}$ and its antagonistic relationship with adiponectin may lead to hypoadiponectineamia in modern high yielding cows. More importantly, hypoadiponectinemia could be another mechanism that contributes to poor fertility in dairy cows. Based on the results of the present study, adiponectin is a plausible regulator of metabolism and reproduction in dairy cows. Nevertheless, the specific role of adiponectin in bovine physiology is yet to be clarified. 


\section{APPENDIX}

\section{A.1. Blood sampling and post-collection processing}

Blood samples for hormonal assays, and plasma metabolites were collected from the coccygeal veins of cows into evacuated $10 \mathrm{ml}$ vacutainer tubes (BD Vacutainer Systems ${ }^{\circledR}$, Belliver Industrial Estate, Plymouth, UK) containing heparin anticoagulant. The blood samples were collected on ice and centrifuged within 10 minutes at $3000 \mathrm{rpm}$ for 15 minutes at $4{ }^{\circ} \mathrm{C}$. Plasma samples was separated into duplicate aliquots and stored frozen in $5 \mathrm{ml}$ screw cap containers at $-20^{\circ} \mathrm{C}$ until subsequently assayed.

\section{A.2. Experimental diets}

The two diets were formulated to be iso-energetic (ME 12.5 MJ/kg DM) and isonitrogenous (CP 180 $\mathrm{g} / \mathrm{kg} \mathrm{DM})$. The only difference between the two diets was that the High Starch diet had higher starch content than the High Fat diet $(182 \mathrm{~g} / \mathrm{kg}$ DM versus 98 $\mathrm{g} / \mathrm{kg} \mathrm{DM})$ and the High Fat diet had higher fat content than the High Starch diet $(53 \mathrm{~g} / \mathrm{kg}$ DM versus $39 \mathrm{~g} / \mathrm{kg} \mathrm{DM})$. 
Table A.1: Formulation and composition of High Starch and High Fat diets (Garnsworthy et al., 2009)

\begin{tabular}{lcc}
\hline \hline & \multicolumn{2}{c}{ DIET } \\
\cline { 2 - 3 } & HIGH STARCH & HIGH FAT \\
[HF]
\end{tabular}

${ }^{\dagger}$ DM, Dry Matter; ME, Metabolisable Energy; CP, Crude Protein; NDF, Neutral Detergent Fibre ${ }^{a}$ Megalac, calcium salts of palm fatty acids; Volac International, Royston, UK.

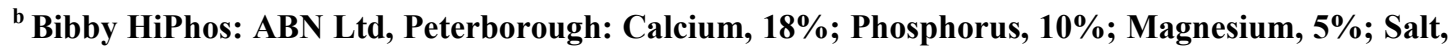
17\%; Copper, $2000 \mathrm{mg} / \mathrm{kg}$; Manganese, $5000 \mathrm{mg} / \mathrm{kg}$; Cobalt, $100 \mathrm{mg} / \mathrm{kg}$; Zinc, $6000 \mathrm{mg} / \mathrm{kg}$; Iodine, $500 \mathrm{mg} / \mathrm{kg}$; Selenium, $25 \mathrm{mg} / \mathrm{kg}$; Vitamin A, 400,000 IU/kg; Vitamin D3 80,000 IU/kg; Vitamin E, $1000 \mathrm{mg} / \mathrm{kg}$. 


\section{A.3. Measurement of adiponectin in blood plasma (Linco Research kit \#HADP-61HK)}

\section{A.3.1. 10x Assay Buffer}

Final concentration upon dilution is $10.0 \mathrm{mM}$ Phosphate Buffer, $\mathrm{pH} 7.6$ containing $0.08 \%$ Sodium Azide,

$0.1 \%$ RIA Grade BSA

Quantity: $50 \mathrm{ml} / \mathrm{vial}$

Preparation: The content of the vial was diluted with $450 \mathrm{ml}$ deionized water.

\section{A.3.2. Antiserum}

Rabbit anti-Adiponectin Antibody

Quantity: $13 \mathrm{ml} / \mathrm{vial}$

Preparation: Ready to use

\section{A.3.3. 125I-Adiponectin}

${ }^{125}$ I-Adiponectin Label (specific activity $67.7 \mu \mathrm{Ci} / \mu \mathrm{g}$ ) lyophilized for stability. Freshly iodinated label contains $<3 \mu \mathrm{Ci},(<111 \mathrm{kBq})$.

Quantity: $13.5 \mathrm{ml} / \mathrm{vial}$ upon hydration

Preparation: ${ }^{125}$ I-Adiponectin was hydrated with $13.5 \mathrm{ml}$ of $1 x$ Assay Buffer and allowed to sit at room temperature for 30 minutes, with occasional gentle mixing.

\section{A.3.4. Standards}

Purified Recombinant Adiponectin, $200 \mathrm{ng} / \mathrm{ml}$ lyophilized for stability Quantity: $1 \mathrm{ml}$ upon hydration Preparation: The Purified recombinant adiponectin was hydrated with $1 \mathrm{ml}$ deionized water.

\section{A.3.5. Quality controls $1 \& 2$}

Purified recombinant adiponectin lyophilized for stability

Quantity: $1 \mathrm{ml} / \mathrm{vial}$ upon hydration 
Preparation: The Purified Recombinant Adiponectin reconstituted with $1 \mathrm{ml}$ deionized water and mixed well.

\section{A.3.6. Rabbit Carrier}

$30 \%$ Normal Rabbit Serum

Quantity: $2 \mathrm{ml} / \mathrm{vial}$

Preparation: Ready to use

\section{A.3.7. Precipitating Reagent (chilled at $4^{\circ} \mathrm{C}$ before being used)}

Goat anti-Rabbit IgG Serum, 3\% PEG and 0.05\% Triton X-100 in 0.05 M Phosphosaline, 0.025 M EDTA,

$0.08 \%$ Sodium Azide

Quantity: $130 \mathrm{ml} /$ vial

Preparation: Ready to use

\section{A.3.8. Preparation of standards}

Standards were prepared in polypropylene tubes on the day of use, as specified in the tables below.

\begin{tabular}{ccc}
\hline \hline $\begin{array}{c}\text { Standards concentration } \\
(\boldsymbol{n g} / \boldsymbol{m} \boldsymbol{l})\end{array}$ & $\begin{array}{c}\text { Volume of } \\
\text { Deiodinized Water } \\
\text { to add }\end{array}$ & $\begin{array}{c}\text { Volume of Standards to } \\
\text { add }\end{array}$ \\
\hline 200 & $1 \mathrm{ml}$ & 0 \\
\hline \hline
\end{tabular}




\begin{tabular}{cccc}
\hline \hline Stds & $\begin{array}{c}\text { Std concentration } \\
(\mathbf{n g} / \mathbf{m l})\end{array}$ & $\begin{array}{c}\text { Volume of Assay } \\
\text { Buffer to add }\end{array}$ & $\begin{array}{c}\text { Volume of Std to add } \\
\text { (Serial Dilutions) }\end{array}$ \\
\hline 1 & 100 & $0.5 \mathrm{ml}$ & $0.5 \mathrm{ml}$ of $200 \mathrm{ng} / \mathrm{ml}$ \\
2 & 50 & $0.5 \mathrm{ml}$ & $0.5 \mathrm{ml}$ of $100 \mathrm{ng} \mathrm{ml}$ \\
3 & 25 & $0.5 \mathrm{ml}$ & $0.5 \mathrm{ml}$ of $50 \mathrm{ng} \mathrm{ml}$ \\
4 & 12.5 & $0.5 \mathrm{ml}$ & $0.5 \mathrm{ml}$ of $25 \mathrm{ng} / \mathrm{ml}$ \\
5 & 6.25 & $0.5 \mathrm{ml}$ & $0.5 \mathrm{ml}$ of $12.5 \mathrm{ng} / \mathrm{ml}$ \\
6 & 3.125 & $0.5 \mathrm{ml}$ & $0.5 \mathrm{ml}$ of $6.25 \mathrm{ng} / \mathrm{ml}$ \\
7 & 1.56 & $0.5 \mathrm{ml}$ & $0.5 \mathrm{ml}$ of $3.125 \mathrm{ng} / \mathrm{ml}$ \\
8 & 0.78 & $0.5 \mathrm{ml}$ & $0.5 \mathrm{ml}$ of $1.56 \mathrm{ng} / \mathrm{ml}$ \\
\hline \hline
\end{tabular}

\section{A.3.9. Assay Procedure}

The Adiponectin assay was run in 2 days as follows:

$\underline{1^{\text {st }} \text { Day: }}$

- Polypropylene tubes were labeled.

- $300 \mu l$ of Assay Buffer pipetted to the Non-Specific Binding (NSB) tubes (3-4).

- $200 \mu l$ of Assay Buffer added into the Reference $\left(B_{0}\right)$ tubes (5-6).

- $100 \mu l$ of Assay Buffer added to tubes seven through the end of the assay.

- $100 \mu l$ of Standards and Quality Controls added in duplicate into tubes.

- $100 \mu l$ of each sample (undiluted) in duplicate pipetted to tubes.

- $100 \mu l$ of ${ }^{125}$ I-Adiponectin pipetted to all tubes.

- $100 \mu l$ of Adiponectin Antibody pipetted to all tubes except Total Count tubes (1-2) and NSB tubes (3-4).

- All tubes were vortexed, covered and incubated overnight (20-24 hours) at room temperature.

$\underline{2^{\text {nd }} \text { Day: }}$

- $10 \mu l$ of Rabbit Carrier pipetted to all tubes except Total Count tubes (1-2).

- $1.0 \mathrm{ml}$ of cold $\left(4^{\circ} \mathrm{C}\right)$ Precipitating Reagent was added to all tubes except Total Count tubes (1-2).

- All tubes were vortexed and incubated 20 minutes at room temperature. 
- All tubes were centrifuged at 3,000 rpm for 30 minutes except Total Count tubes (1-2).

- The supernatant was decanted immediately from all tubes except TC tubes.

- Tubes left to drain for 60 seconds and the pellet counted for 1 minute on the gamma counter.

Results were calculated automatically by the instrument software (Multicalc, Wallac, Stockholm, Sweden).

The assays run five times and a composite standard curve (a plot of $\mathrm{B} / \mathrm{B}_{0}$ against adiponectin concentration) was created (Figure A.1). Sensitivity of the assay, expressed as effective dose $80 \%\left(\mathrm{ED}_{80}\right)$, was calculated from this composite standard curve, and it was $2.33 \pm 0.08 \mathrm{ng} / \mathrm{ml}$.

Serial dilution of bovine plasma $(1 / 2,1 / 3,1 / 5,1 / 7$, and 1/10) led to samples contained adiponectin concentrations below the sensitivity of the assay. Thus, validation of the method by showing parallelism was not feasible.

\section{A.3.10. Equipment}

11277 Gammamaster, LKB, Wallac, Stockholm, Sweden.

2. Jouan KR422 Centrifuge. 


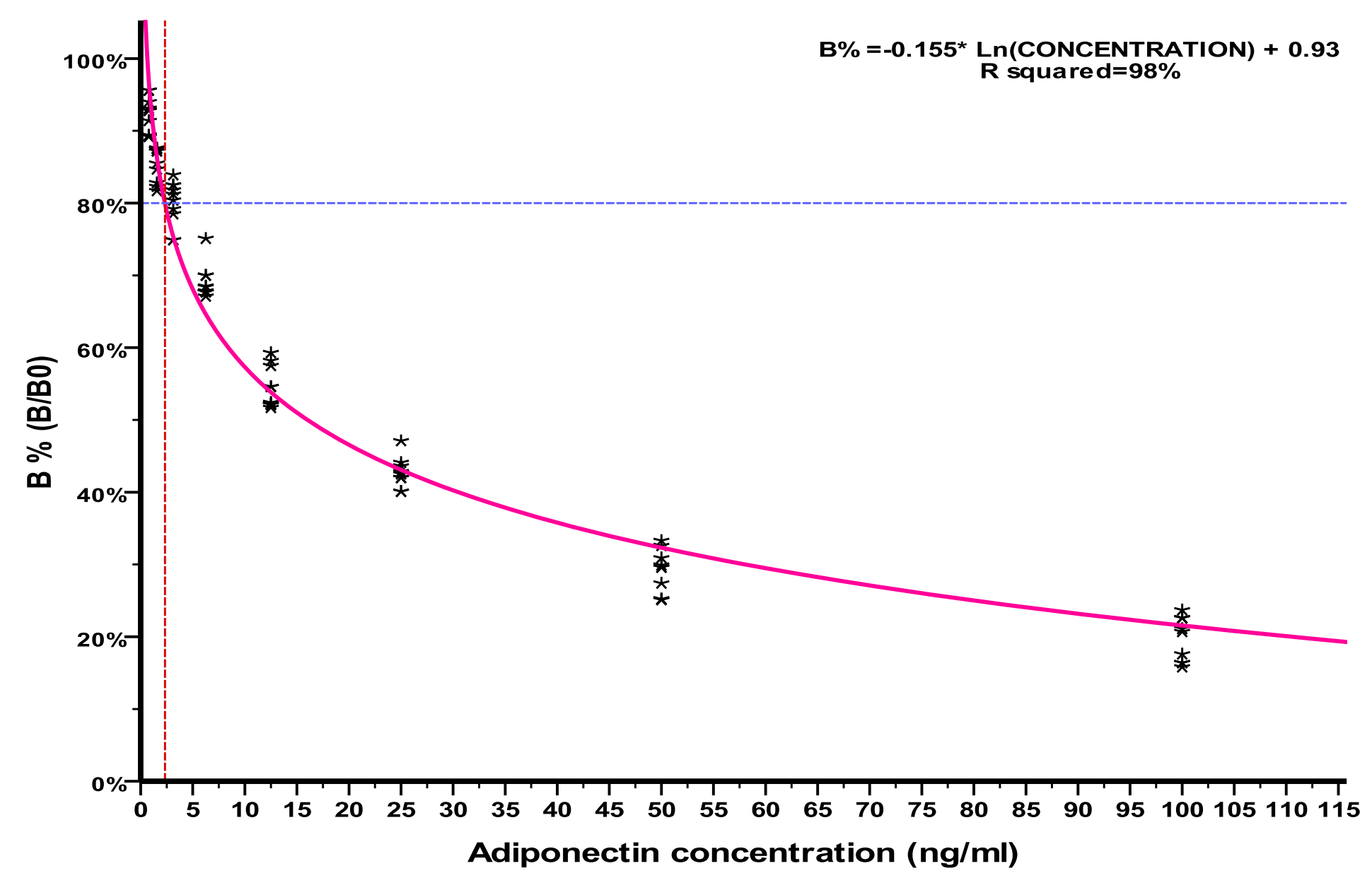

Figure A.1: Composite standard curve of plasma adiponectin assay. A plot of $\mathrm{B} / \mathrm{B}_{0}$ against adiponectin concentration was created for five consequtive runs of plasma adiponectin assay. Sensitivity of the assay, expressed as effective dose $80 \%$ (ED $\left.\mathrm{E}_{80}\right)$, was calculated from this standard curve, and it was $2.33 \mathrm{ng} / \mathrm{ml}$ 


\section{A.4. Measurement of adiponectin in bovine milk (Linco Research kit \#HADP-61HK)}

Milk samples were collected from 6 cows in different stage of lactation. All samples were collected between 15:00 and 17:00 in the afternoon and left in the fridge ( 0 to $\left.4{ }^{\circ} \mathrm{C}\right)$ over night. Because lipids interfere with radioimmunoassay (RIA), skim milk was used. $4 \mathrm{ml}$ of whole milk of each cow was added in 6 tubes. Milk samples were vortexed and skim milk (aqueous phase) was obtained by centrifugation $\left(1500 \mathrm{~g}, 20 \mathrm{~min}, 4^{\circ} \mathrm{C}\right)$, after which the fat layer was removed and weighted in electronic balance (Mettler Toledo, Leicester, UK). Immunoreactive adiponectin was assessed in duplicate by using a commercial RIA kit (Linco Research, St Charles, MO) with the use of $100 \mu l$ of skim milk. The adiponectin kit assay performed as described previously and adiponectin concentration in skim milk was measured.

The assays run one time and a standard curve (a plot of $\mathrm{B} / \mathrm{B}_{0}$ against adiponectin concentration) was created (Figure A.2). Sensitivity of the assay, expressed as effective dose $80 \%\left(\mathrm{ED}_{80}\right)$, was calculated from this standard curve, and it was $2.14 \mathrm{ng} / \mathrm{ml}$.

Serial dilution of bovine fat free milk $(1 / 2,1 / 3$, and 1/5) led to samples contained adiponectin concentrations below the sensitivity of the assay. Thus, validation of the method by showing parallelism was not possible. 


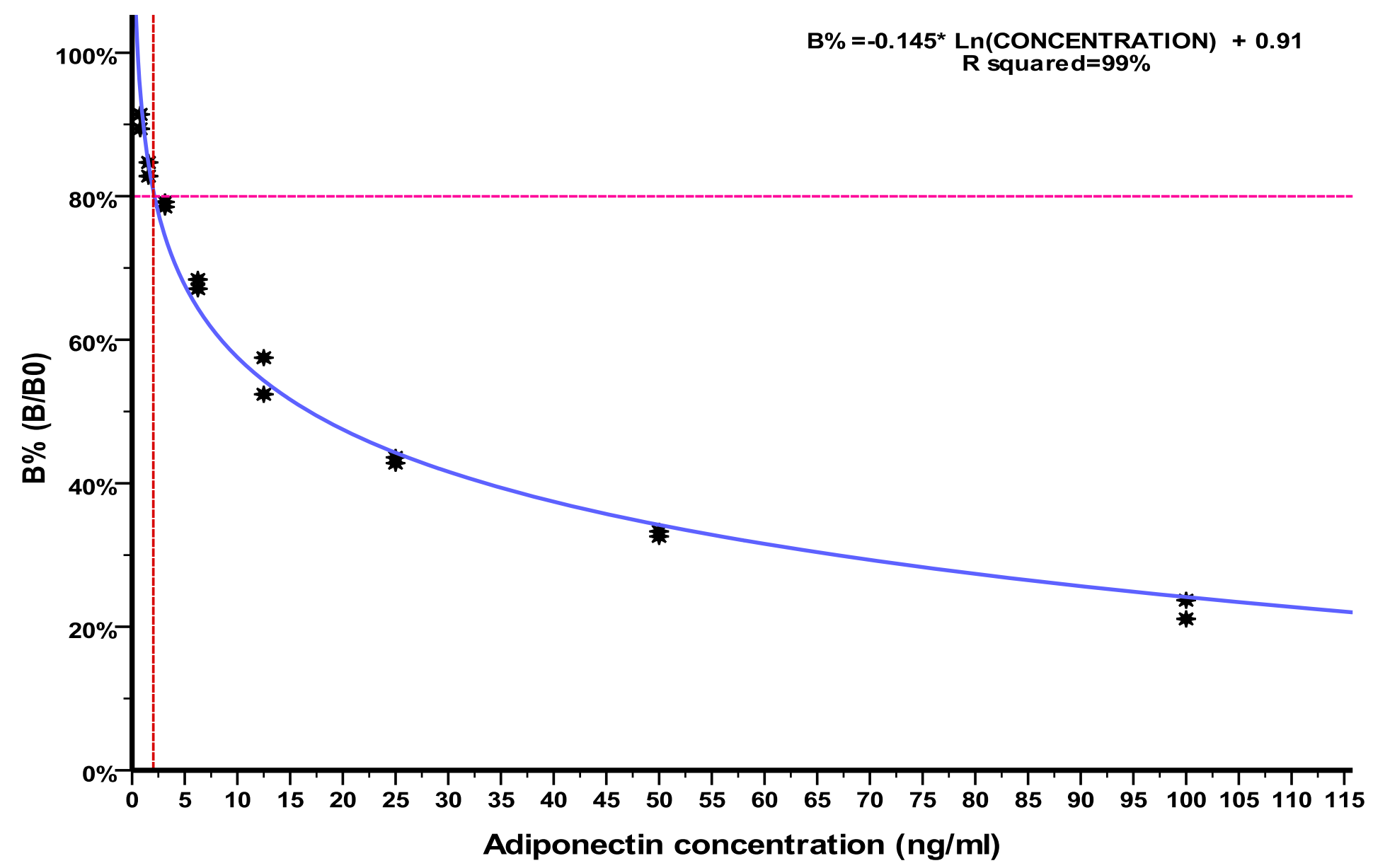

Figure A.2: Standard curve of milk adiponectin assay. A plot of $\mathrm{B} / \mathrm{B}_{0}$ against adiponectin concentration was created for one run of milk adiponectin assay. Sensitivity of the assay, expressed as effective dose $80 \%\left(\mathrm{ED}_{80}\right)$, was calculated from this standard curve, and it was 2.14 $n g / m l$. 


\section{A.5. Measurement of milk progesterone (ELISA kit, Ridgeway Scientific) and determination of milk progesterone profile}

Whole milk samples were analyzed for progesterone concentrations using a microtitre plate enzyme-linked immunosorbant assay (ELISA) kit (Ridgeway Scientific, Alvington, UK), validated by Sauer et al. (1986).

Samples were removed from the refrigerator (stored at $4^{\circ} \mathrm{C}$ after addition of Lactab MkIII tablets) at least 3 hours before analysis and warmed to $25^{\circ} \mathrm{C}$. The plates are also brought at room temperature, the foil stripped from the wells and the wells emptied and tapped dry. To each well $10 \mu \mathrm{l}$ of sample or standard (1 to $10 \mathrm{ng} / \mathrm{ml}$ progesterone in milk from an oestrus animal) was added, followed by $200 \mu l$ of progesterone-enzyme label (progesterone- $11-\alpha$ glucuronide-alkaline phosphatase). The plate was then vortexed and left for $1-1.5$ hours at room temperature.

After incubation the plate was washed three times with cold water and tapped dry each time. Finally $200 \mu l$ of substrate in substrate buffer was added, and the plates vortexed again and left for developing the color. The samples were read on a $570 \mathrm{~nm}$ plate reader. Quality control samples could not be stored for the entire period of the trial. New quality control samples were selected from previously analyzed samples.

The reliable reading range of the ELISA was from 1.5 to $10.5 \mathrm{ng} / \mathrm{ml}$. Samples reading < $1.5 \mathrm{ng} / \mathrm{ml}$ were taken as $1.5 \mathrm{ng} / \mathrm{ml}$; samples reading $>10.5 \mathrm{ng} / \mathrm{ml}$ were diluted to bring the reading within range. If the coefficient of variation $(\mathrm{CV})$ of duplicate sample readings was $>15 \%$, the analysis was repeated. The intra- and inter-assay coefficients of variation were $<15$ and $6.6 \%$, respectively.

Milk progesterone was measured twice in a week (either Monday and Thursday or Tuesday and Friday mornings) and a rise in progesterone was defined as above $3 \mathrm{ng} / \mathrm{ml}$ for two consecutive samples (Lamming \& Darwash, 1998; Garnsworthy et al., 2009). 
Progesterone profiles were used to classify estrous cycles as normal or abnormal (DOV1, DOV2, PCL1 or PCL2), following the definitions of Lamming \& Darwash (1998) (Table A.2).

Table A.2: Definition of atypical ovarian activity in dairy cattle using milk progesterone profile (Lamming \& Darwash, 1998)

\begin{tabular}{|c|c|}
\hline Atypical reproductive pattern & Definition \\
\hline $\begin{array}{l}\text { Delayed ovulation type I } \\
\text { (DOV1) }\end{array}$ & $\begin{array}{r}\text { Milk P4 concentration }<3 \mathrm{ng} / \mathrm{ml} \text { for } \geq \\
45 \text { days postpartum }\end{array}$ \\
\hline $\begin{array}{l}\text { Prolonged inter-luteal interval, delayed } \\
\text { ovulation type II } \\
\text { (DOV2) }\end{array}$ & $\begin{array}{l}\text { Milk P4 concentration }<3 \mathrm{ng} / \mathrm{ml} \text { for } \geq \\
12 \text { days between two luteal phases. }\end{array}$ \\
\hline $\begin{array}{l}\text { Delayed luteolysis during first cycle, } \\
\text { persistent CL type I } \\
\text { (PLC1) }\end{array}$ & $\begin{array}{r}\text { Milk P4 concentration }>3 \mathrm{ng} / \mathrm{ml} \text { for } \geq 19 \\
\text { days during the first postpartum } \\
\text { oestrous cycle. }\end{array}$ \\
\hline $\begin{array}{c}\text { Delayed luteolysis during subsequent } \\
\text { cycles before insemination, persistent } \\
\text { CL type II (PCL2) }\end{array}$ & $\begin{array}{r}\text { Milk P4 concentration }>3 \mathrm{ng} / \mathrm{ml} \text { for } \geq 19 \\
\text { days during subsequent postpartum } \\
\text { cycles. }\end{array}$ \\
\hline
\end{tabular}




\section{A.6. Measurement of metabolites in blood plasma}

Blood plasma samples were analyzed for the following metabolites on a Bayer opera autoanalyzer (Bayer UK Ltd).

\section{A.11.1. Urea}

Plasma Urea concentrations were determined in a single assay, using a kit supplied by Bayer (Bayer kit T01 182356). The method was based on Urea hydrolyzation in the presence of water and urease. The coefficient of variation was less than $5 \%$.

\section{A.11.2. Glucose}

Plasma glucose concentrations were determined in a single assay using a kit supplied by Bayer (Bayer kit T01 183356). The method was based on hexokinase catalytic phosphorylation of plasma glucose. The coefficient of variation for samples was less than $5 \%$.

\section{A.11.3. $\beta$-hydroxybutyrate (BOHB)}

Plasma $\beta$-hydroxybutyrate concentrations were determined using an enzymatic kit supplied by Randox (Ranbut RB 1008). The method was based on the oxidation of D-3hydroxybutyrate to acetoacetate by the enzyme 3-hydroxybutyrate dehydrogenase. The coefficient of variation for samples was less than $5 \%$.

\section{A.11.4. Non- esterified fatty acids (NEFA)}

Plasma NEFA concentrations were determined using an enzymatic kit supplied Waiko (Waiko kit NEFA-C). The method was based on the acylation of coenzyme A by the fatty acids in the presence of added acyl-CoA synthetase. The coefficient of variation for samples was less than $5 \%$. 
B.1. Parameter estimates of models were used to explore interrelationships of adiponectin with glucose, BCS, metabolic hormones, and metabolites (Chapter 5)

Table B.1: Model parameter estimates

TYPE OF MODEL

\begin{tabular}{|c|c|c|c|}
\hline Estimates: & BASIC MODEL ${ }^{\dagger}$ & LOW PMY ${ }^{\dagger}$ & HIGH PMY ${ }^{\dagger}$ \\
\hline $\begin{array}{l}\text { Glucose } \\
(\mathrm{mmol} / \mathrm{l})\end{array}$ & $3.40 \pm 0.478$ & $3.38 \pm 0.551$ & $3.42 \pm 0.626$ \\
\hline $\begin{array}{l}\text { Insulin (INS) } \\
\quad(n g / m l)\end{array}$ & $0.41 \pm 0.012$ & $0.42 \pm 0.019$ & $0.40 \pm 0.015$ \\
\hline $\begin{array}{c}\text { Glucagon (GLGON) } \\
(p g / m l)\end{array}$ & $95.1 \pm 2.47$ & $98.9 \pm 3.51$ & $91.2 \pm 3.3$ \\
\hline $\begin{array}{l}\text { Adiponectin (ADP) } \\
(n g / m l)\end{array}$ & $8.5 \pm 0.72$ & $9.9 \pm 1.15$ & $7.2 \pm 0.82$ \\
\hline $\begin{array}{c}\text { Leptin (LPN) } \\
(n g / m l)\end{array}$ & $1.73 \pm 0.115$ & $1.9 \pm 0.203$ & $1.57 \pm 0.106$ \\
\hline$\underset{(n g / m l)}{\mathbf{G H}}$ & $4.55 \pm 0.266$ & $4.16 \pm 0.37$ & $4.95 \pm 0.394$ \\
\hline $\begin{array}{c}\text { BOHB } \\
(\mathrm{mmol} / \mathrm{l})\end{array}$ & $0.65 \pm 0.029$ & $0.60 \pm 0.032$ & $0.69 \pm 0.049$ \\
\hline $\begin{array}{c}\text { NEFA } \\
(\mathrm{mmol} / \mathrm{l})\end{array}$ & $0.43 \pm 0.033$ & $0.45 \pm 0.057$ & $0.40 \pm 0.036$ \\
\hline $\begin{array}{c}\text { BCS } \\
\text { (units 1-5) }\end{array}$ & $2.76 \pm 0.037$ & $2.76 \pm 0.056$ & $2.77 \pm 0.052$ \\
\hline
\end{tabular}

$\dagger$ Columns are means \pm SE. 


\section{List of references}

Adamiak SJ, Mackie K, Watt RG, Webb R \& Sinclair KD, 2005. Impact of nutrition on oocyte quality: Cumulative effects of body composition and diet leading to hyperinsulinemia in cattle. Biology of Reproduction 73: 918-926.

Adamiak SJ, Powell K, Rooke JA, Webb R ,\& Sinclair KD, 2006. Body composition, dietary carbohydrates and fatty acids determine post-fertilisation development of bovine oocytes in vitro. Reproduction 131: 247-258.

Adamiak SJ, 2005. The nutritional regulation of oocyte quality and early embryo development in cattle. PhD Thesis. University of Nottingham, UK.

Adashi EY, Hsueh AJW, \& Yen SSC, 1981. Insulin enhancement of luteinising hormone and follicle stimulating hormone release by cultured pituitary cells. Endocrinology 108: 1441-1449.

Agenas S, Burstedt E, \& Holtenius K, 2003. Effects of feeding intensity during the dry period. 1. Feed intake, body weight, and milk production. J Dairy Sci. 86:870-882.

Agresti A, 1996. An introduction in categorical data analysis. In Wiley series in probability and statistics (eds: Barnett V, Bradley RA, Fisher NI, Hunter JS, Kadane JB, Kendall DG, Scott DW, Smith AFM, Teugels JL, \& Watson GS). John Wiley \& sons Inc, NY, USA.

Ahima RS, 2006. Metabolic actions of adipocyte hormones: Focus on adiponectin. Obesity 14 (Suppl February): 9S-15S.

Ahima RS, Dushay J, Flier SN, Prabakaran D, \& Flier JS, 1997. Leptin accelerates the onset of puberty in normal female mice. J. Clin. Invest 99 (3): 391-395.

Albert PS, 1999. Longitudinal data analysis (repeated measures) in clinical trials. Statist. Med. 18:1707-1732.

Allen MS, Bradford BJ, \& Oba M, 2009. The hepatic oxidation theory of the control of feed intake and its application to ruminants. J Anim. Sci. 87: 3317-3334.

Allen MS \& Bradford BJ, 2007. Regulation of feed intake in transition cows: Application of the hepatic oxidation hypothesis. $22^{\text {nd }}$ Annual Southwest Nutrition and Management Conference, February 22-23, Tempe, 138-146. 
Allen MS, Bradford BJ, \& Harvatine KJ, 2005. The cow as a model to study food intake regulation. Annu. Rev. Nutr. 25: 523-547.

Allison PD, 2001. Missing data. Sage, Thousand Oaks, UK.

Ametaj BN, 2005. A new understanding of the causes of fatty liver in dairy cows. Advances in Dairy Technology 17: 97-112.

Andersson U, Filipsson K, Abbott CR, Woods A, Smith K, Bloom SR, Carling D, \& Small CJ, 2004. AMP-activated protein kinase plays a role in the control of food intake. The Journal of Biological Chemistry 279 (13): 12005-12008.

Antuna-Puente B, Feve B, Fellahi S, \& Bastard JP, 2008. Adipokines: The missing link between insulin resistance and obesity, Diabetes \& Metabolism 34: 2-11.

AOAC, 1990. Official methods of analysis. 15 edition, Arlington, VA: Association of official analytical chemists.

Ardawi MSM, Path FRC,\& Rouzi AA, 2005. Plasma adiponectin and insulin resistance in women with polycystic ovary syndrome. Fertility \& Sterility 83(6):1708-1716.

Arias P, Rodriguez M, Szwarcfar B, Sinay IR, \& Moguilevsky JA, 1992. Effect of insulin on LHRH release by perifused hypothalamicfragments. Neuroendocrinology 56: 415-418.

Aronoff SL, Berkowitz K, Shreiner B, \& Want L, 2004. Glucose metabolism and regulation: beyond insulin and glucagon. Diabetes Spectrum 17: 183-190.

Asai-Sato M, Okamoto M, Endo M, Yoshida H, Murase M, Ikeda M, Sakakibara H, Takahashi T \& Hirahara F, 2006. Hypoadiponectinemia in lean lactating women: Prolactin inhibits adiponectin secretion from human adipocytes. Endocrine Journal 53 (4): $555-562$.

Baldi A, Cheli F, Pinotti L, \& Pecorini C, 2008. Nutrition in mammary gland health and lactation: Advances over eight biology of lactation in farm animals meetings. J Anim. Sci. 86 (Suppl. 1): 3-9.

Balen A, 2001. Hypersecretion of LH: effects and mechanisms. In polycystic ovary syndrome (ed: Homburg R), Martin Dunitz Ltd, London, UK. pp. 61-78.

Ball PJH \& Peters AR, 2004. Reproduction in Cattle. Blackwell Publishing Ltd, Oxford, UK. 
Barb D, Williams CJ, Neuwirth AK, \& Mantzoros CS, 2007. Adiponectin in relation to malignancies: a review of existing basic research and clinical evidence. Am. J Clin. Nutr. 86 (suppl.): $858 \mathrm{~S}-866 \mathrm{~S}$.

Barbour LA, McCurdy CE, Hernandez TL, Kirwan JP, Catalano PM, \& Friedman JE, 2007. Cellular mechanisms for insulin resistancein normal pregnancy and gestational diabetes. Diabetes Care 30 (Suppl. 2): 112-119.

Barnea M, Shamay A, Stark AH, \& Madar Z, 2006. A High-Fat diet has a tissuespecific effect on adiponectin and related enzyme expression. Obesity 14 (12): 2145-2153.

Barnes MA, Kazmer GW, Akers RM, \& Pearson RE, 1985. Influence of selection for milk yield on endogenous hormones and metabolites in holstein heifers and cows. J Anim. Sci. 60: 271-284.

Basu S, Laffineuse L, Presley L, Minium J, Catalano PM, \& Hauguel-de Mouzon S 2009. In utero gender dimorphism of adiponectin reflects insulin sensitivity and adiposity of the fetus. Obesity 17(6): 1144-1149.

Bauman DE \& Currie WB, 1980. Partitioning of nutrients during pregnancy and lactation: a review of mechanisms involving homeostasis and homeorhesis. J Dairy Sci. 63:1514-1529.

Bauman DE \& Griinari JM, 2000. Historical perspective and recent developments in identifying the cause of diet-induced milk fat depression. Proc. Cornell Nutr. Conf. pp. 191-202.

Bauman DE, 1999. Bovine somatotropin and lactation: from basic science to commercial application. Domestic Animal Endocrinology 17: 101-116.

Bauman DE, 2000. Regulation of nutrient partitioning during lactation: homeostasis and homeorhesis revisited. In Ruminant physiology: digestion, metabolism, growth and reproduction (ed.: Cronje PB). CABI Publishing, Wallingford, UK. pp. 311-328.

Bauman DE, Lock AL, Baumgard LH, \& Collier RC, 2004. Nutrient partitioning and milk yield: constraints and opportunities in the 21 st century. Proc. Cornell Nutr. Conf. pp. 107-121.

Beam SW \& Butler WR, 1997. Energy balance and ovarian follicle development prior to the first ovulation postpartum in dairy cows receiving three levels of dietary fat. Biology of Reproduction 56: 133-142.

Beam SW \& Butler WR, 1999. Effects of energy balance on follicular development and first ovulation in postpartum dairy cows, J Reprod. Fertil. Suppl. 54: 411-424. 
Beever DE, 2006. The impact of controlled nutrition during the dry period on dairy cow health, fertility and performance. Animal Reproduction Science 96: 212-226.

Bell AW \& Bauman DE, 1997. Adaptations of glucose metabolism during pregnancy and lactation. Journal of Mammary Gland Biology and Neoplasia 2(3): 265-278.

Bell AW, 1995. Regulation of organic nutrient metabolism during transition from late pregnancy to early lactation. J Anim. Sci. 73: 2804-2819.

Ben-Shlomo I, 2002. The polycystic ovary syndrome: what does insulin resistance have to do with it? Reproductive BioMedicine 6(1): 36-42.

Berg AH, Combs TP \& Scherer PE, 2002. ACRP30/adiponectin: an adipokine regulating glucose and lipid metabolism. TRENDS in Endocrinology \& Metabolism 13 (2): 84-89.

Berry DP, Buckley F, Dillon P, Evans RD, Rath M, \& Veerkamp RF, 2003. Genetic relationships among body condition score, body weight, milk yield, and fertility in dairy cows. J Dairy Sci. 86: 2193-2204.

Bewley JM \& Schutz MM, 2008. Review: An interdisciplinary review of body condition scoring for dairy cattle. The Professional Animal Scientist 24: 507-529.

Bjursell M, Ahnmark A, Bohlooly-Y M, William-Olsson L, Rhedin M, Peng XR, Ploj K, Gerdin AK, Arnerup G, Elmgren A, Berg AL, Oscarsson J, \& Linden D, 2007. Opposing effects of adiponectin receptors 1 and 2 on energy metabolism. Diabetes 56: 583-593.

Block SS, Butler WR, Ehrhardt RA, Bell AW, Van Amburgh ME \& Boisclair YR, 2001. Decreased concentration of plasma leptin in periparturient dairycows is caused by negative energy balance. Journal of Endocrinology 171: 339-348.

Block SS, Rhoads RP, Bauman DE, Ehrhardt RA, McGuire MA, Crooker BA, Griinari JM, Mackle TR, Weber WJ, Van Amburgh ME, \& Boisclair YR, 2003. Demonstration of a role for insulin in the regulation of leptin in lactating dairy cows, $J$ Dairy Sci. 86: 3508-3515.

Bobe G, Ametaj BN, Young JW, \& Beitz DC, 2003a. Effects of exogenous glucagon on lipids in lipoproteins and liver of lactating dairy cows. J Dairy Sci. 86: 2895-2903.

Bobe G, Ametaj BN, Young JW, \& Beitz DC, 2003b. Potential treatment of fatty liver with 14-day subcutaneous injections of glucagon. J Dairy Sci. 86: 3138-3147.

Bobe G, Ametaj BN, Young JW, Anderson LL, \& Beitz DC, 2007. Exogenous glucagon effects on health and reproductive performance of lactating dairy cows with mild fatty liver. Animal Reproduction Science 102: 194-207. 
Bobbert T, Rochlitz H, Wegewitz U, Akpulat S, Mai K, Weickert MO, Mohlig M, Pfeiffer AFH,\& Spranger J, 2005. Changes of adiponectin oligomer composition by moderate weight eduction. Diabetes 54: 2712-2719.

Bobe G, Young JW, \& Beitz DC, 2004. Invited Review: Pathology, etiology, prevention, and treatment of fatty liver in dairy cows. J Dairy Sci. 87: 3105-3124.

Boelhauve M, Sinowatz F, Wolf E, \& Paula-Lopes FF, 2005. Maturation of bovine oocytes in the presence of leptin improves development and reduces apoptosis of in vitroproduced blastocysts. Biology of Reproduction 73: 737-744.

Boland MP \& Lonergan P, 2003. Effects of nutrition on fertility in dairy cows. Advances in Dairy Technology 15: 19-27.

Boland MP, Lonergan P \& O'Callaghan D, 2001. Effect of nutrition on endocrine parameters, ovarian physiology, and oocyte and embryo development. Theriogenology 55: $1323-1340$.

Bonnet M, Delavaud C, Laud K, Gourdou I, Leroux C, Djiane J, \& Chilliard Y, 2002. Mammary leptin synthesis, milk leptin and their putative physiological roles. Reprod. Nutr. Dev. 42: 399-413.

Borromeo V, Berrini A, Bramani S, Sironi G, Finazzi M,\& Secchi C,1998. Plasma levels of GH and PRL and concentrations in the fluids of bovine ovarian cysts and follicles. Theriogenology 49:1377-1387.

Bossaert P, Leroy JLMR, De Campeneere S, De Vliegher S \& Opsomer G, 2009. Differences in the glucose-induced insulin response and the peripheral insulin responsiveness between neonatal calves of the Belgian Blue, Holstein-Friesian, and East Flemish breeds, J Dairy Sci. 92: 4404-4411.

Bossaert P, Leroy JLMR, De Vliegher S, \& Opsomer G, 2008. Interrelations between glucose-induced insulin response, metabolic indicators, and time of first ovulation in highyielding dairy cows. J Dairy Sci. 91: 3363-3371.

Bousquet D, Bouchard E, \& DuTremblay D, 2004. Decreasing Fertility in Dairy Cows: Myth or Reality? Proceedings of the WBC Congress, $23^{\text {rd }}$ World Buiatrics Congress, Quebec City, Canada.

Brochu-Gaudreau K, Rehfeldt C, Blouin R, Bordignon V, Murphy BD,\& Palin MF, 2010. Adiponectin action from head to toe. Endocrinology 37: 11-32.

Brockman RP \& Laarveld B, 1986. Hormonal regulation of metabolism in ruminants:a review. Livestock Production Science 14: 313-334. 
Brockman RP, 1978. Roles of glucagon and insulin in the regulation of metabolism in ruminants. Canadian Veterinary Journal 19: 55-62.

Brockman RP, 1979. Roles for insulin and glucagon in the development of ruminant ketosis. Canadian Veterinary Journal 20: 121-126.

Bronsky J, Karpisek M, Bronska E, Pechova M , Jancikova B, Kotolova H, Stejskal D, Prusa Richard, \& Nevoral J, 2006. Adiponectin, Adipocyte Fatty Acid Binding Protein, and Epidermal Fatty Acid Binding Protein: Proteins Newly Identified in Human Breast Milk. Clinical Chemistry 52: (9)1763-1770.

Broster WH \& Broster VJ, 1998. Body score of dairy cows. J. Dairy Res. 65: 155-173.

Bruning JC, Gautam D, Burks DJ, Gillette J, Schubert M, Orban PC, Klein R, Krone W, Muller-Wieland D, \& Kahn CR, 2000. Role of brain insulin receptor in control of body weight and reproduction. Science 289: 2122-2125.

Brunson BL, Zhong Q, \& Clarke KJ, 2007. Serum concentrations of adiponectin and characterization of adiponectin protein complexes in dogs. Am J Vet Res 68: 57-62.

Bucholtz DC, Chiesa A, Pappano WN, Nagatani S, Tsukamura H, Maeda KI, \& Foster DL, 2000. Regulation of pulsatile luteinizing hormone secretion by insulin in the diabetic male lamb. Biology of Reproduction 62: 1248-1255.

Buckley F, O'Sullivan K, Mee JF, Evans RD, \& Dillon P, 2003. Relationships among milk yield, body condition, cow weight, and reproduction in spring-calved holsteinfriesians. J Dairy Sci. 86: 2308-2319.

Busato A, Faissler D, Kupfer U, \& Blum JW, 2002. Body condition scores in dairy cows: associations with metabolic and endocrine changes in healthy dairy cows. $J$ Vet. Med. A 49: 455-460.

Butler WR \& Smith RD, 1997. Interrelationships between energy balance and postpartum reproductive function in dairy cattle. J Dairy Sci. 72: 767-783.

Butler WR, 2000. Nutritional interactions with reproductive performance in dairy cattle, Animal Reproduction Science 60-61: 449-457.

Butler WR, 2003. Energy balance relationships with follicular development, ovulation and fertility in postpartum dairy cows. Livestock Production Science 83: 211-218.

Campos DB, Palin MF, Bordignon V, \& Murphy BD, 2008. The beneficial adipokines in reproduction and fertility. International Journal of Obesity 32: 223-231. 
Carmen GY \&Victor SM, 2006. Signaling mechanisms regulating lipolysis. Cellular Signaling 18:401-408.

Casanueva FF \& Diéguez C, 1998. Interaction between body composition, leptin and growth hormone status. Bailliere's Clinical Endocrinology \& Metabolism 12(2): 297-314.

Cassidy A, Skidmore P, Rimm EB, Welch A, Fairweather-Tait S, Skinner J, Burling K, Richards JB, Spector TD, \& MacGregor AJ, 2009. Plasma adiponectin concentrations are associated with body composition and plant-based dietary factors in female twins. $J$ Noutr. 139: 353-358.

Catalano PM, Hoegh M, Minium J, Huston-Presley L, Bernard S, Kalhan S, \& Hauguel-De Mouzon S, 2006. Adiponectin in human pregnancy: implications for regulation of glucose and lipid metabolism. Diabetologia 49: 1677-1685.

Chabrolle C, Tosca L, \& Dupont J, 2007b. Regulation of adiponectin and its receptors in rat ovary by human chorionic gonadotrophin treatment and potential involvement of adiponectin in granulosa cell steroidogenesis. Reproduction 133: 719-731.

Chabrolle C, Tosca L, Crochet S, Tesseraud S, \& Dupont J, 2007a. Expression of adiponectin and its receptors (AdipoR1 and AdipoR2) in chicken ovary: potential role in ovarian steroidogenesis. Domest. Anim. Endocrinol. 33: 480-487.

Chabrolle C, Tosca L, Ram C, Lecomt P, Roy D, \& Dupont J, 2009. Adiponectin increases insulin-like growth factor I-induced progesterone and estradiol secretion in human granulosa cells. Fertility \& Sterility 92(6): 1988-1996.

Chagas LM, Bass JJ, Blache D, Burke CR, Kay JK, Lindsay DR, Lucy MC, Martin GB, Meier S, Rhodes FM, Roche JR, Thatcher WW, \& Webb R, 2007. New perspectives on the roles of nutrition and metabolic priorities in the subfertility of highproducing dairy cows. J Dairy Sci. 90: 4022-4032.

Chagas LM, Lucy MC, Back PJ, Blache D, Lee JM, Gore PJS, Sheahan AJ, \& Roche JR, 2009. Insulin resistance in divergent strains of Holstein-Friesian dairy cows offered fresh pasture and increasing amounts of concentrate in early lactation. J Dairy Sci. 92: 216222.

Chappaz E, Albornoz MS, Campos D, Chea L, Palin MF, Murphy BD, \& Bordignon V, 2008. Adiponectin enhances in vitro development of swine embryos. Domestic Animal Endocrinology 35: 198-207.

Cnaan A, Laird NM, \& Slasor P, 1997. Using the general linear mixed model to analyse unbalanced repeated measures and longitudinal data. Statistics in Medicine 16: 2349-2380. 
Chehab FF, Mounzih K, Lu R, \& Lim ME, 1997. Early onset of reproductive function in normal female mice treated with leptin. Science 273: 88-90.

Chilliard Y, 1992. Physiological constraints to milk production: Factors which determine nutrient partitioning, lactation persistency, and mobilization of body reserves. World Review of Animal Production 27, pp 20-26.

Chilliard Y, Delavaud C, \& Bonnet M, 2005. Leptin expression in ruminants: Nutritional and physiological regulations in relation with energy metabolism. Domestic Animal Endocrinology 29: 3-22.

Ciccioli NH, Wettemann RP, Spicer LJ, Lents CA, White FJ, \& DH Keisler, 2003. Influence of body condition at calving and postpartum nutrition on endocrine function and reproductive performance of primiparous beef cows. J Anim. Sci. 81:3107-3120.

Coffey MP, Simm G, Oldham JD, Hill WG, \& Brotherstone S, 2004. Genotype and diet effects on energy balance in the first three lactations of dairy cows. $J$ Dairy Sci. 87: 4318-4326.

Collier RJ, Bauman DE, \& Hays RL, 1977. Lactogenesis in explant cultures of mammary tissue from pregnant cows. Endocrinology 100: 1192-1200.

Collier RJ, Beede DK, Thatcher WW, Israel LA \& Wilcox CJ, 1982. Influences of environment and its modifications on dairy animal health and production. $J$ Dairy Sci. 65: 2213-2227.

Combs TP, Berg AH, Obici S, Scherer PE,\& Rossetti L, 2001. Endogenous glucose production is inhibited by the adipose-derived protein Acrp30. The Journal of Clinical Investigation 108 (12): 1885-1891.

Combs TP, Berg AH, Rajala MW, Klebanov S, Iyengar P, Jimenez- Chillaron JC, Patti ME, Klein SL, Weinstein RS, \& Scherer PE, 2003. Sexual differentiation, pregnancy, calorie restriction, and aging affect the adipocyte-specific secretory protein adiponectin. Diabetes 52: 268-276.

Combs TP, Pajvani UB, Berg AH, Lin Y, Jelicks LA, Laplante M, Nawrocki AR, Rajala MW, Parlow AF, Cheeseboro L, Ding YY, Russell RG, Lindemann D, Hartley A, Baker GR, Obici S, Deshaies Y, Ludgate M, Rossetti L, \& Scherer PE, 2004. A transgenic mouse with a deletion in the collagenous domain of Adiponectin displays elevated circulating adiponectin and improved insulin sensitivity. Endocrinology 145: 367383.

Comuzzie AG, Funahashi T, Sonnenberg G, Martin LJ, Jacob HJ, Black AE, Maas D, Takahashi M, Kihara S, Tanaka S, Matsuzawa Y, Blangero J, Cohen D, \& Kissebah A, 2001. The genetic basis of plasma variation in adiponectin, a global 
endophenotype for obesity and the metabolic syndrome. J. Clin. Endocrinol. Metab. 86: 4321-4325.

Cone RD \& EImquist JK, 2008. Neuroendocrine control of energy stores. In Williams Textbook of Endocrinology (eds.: Kronenberg HM, Melmed S, Polonsky K, \& Larsen PR), Edition $11^{\text {th }}$ Saunders Elsevier. pp. 1537-1561.

Crooker BA, Carriquiry M, \& Weber WJ, 2007. Energy balance of the periparturient dairy cow. Four-state dairy nutrition and management conference. June 13 \& 14, 2007. Dubuque, Iowa, USA. pp 1-6.

Cryer PE, 2008. Glucose homeostasis and hypoglycemia. In Williams Textbook of Endocrinology (eds.: Kronenberg HM, Melmed S, Polonsky K, \& Larsen PR), Edition $11^{\text {th }}$, Saunders Elsevier, pp 1503-1533.

Davidson MB, 1987. Effect of growth hormone on carbohydrate and lipid metabolism. Endocr Rev. 8(2): 115-131.

Davis CS, 2002. Statistical methods for the analysis of repeated measurements. $1^{\text {st }}$ Edition (eds: Casella G, Fienberg S \& Olkin I), Springer texts in statistics, Springer-Verlag New York, Inc., USA.

De Boer G, Trenkle A, \& Young JW, 1985. Glucagon, insulin, growth hormone, and some blood metabolites during energy restriction ketonemia of lactating cows. J Dairy Sci. 68(2):326-337.

De Boer G, Trenkle A, \& Young JW, 1986. Secretion and clearance rates of glucagon in dairy cows. J Dairy Sci. 69:721-733.

De Vries MJ \& Veerkamp RF, 2000. Energy balance of dairy cattle in relation to milk production variables and fertility, $J$ Dairy Sci. 83: 62-69.

Debras E, Grizard J, Aina E, Tesseraud S, Champredon C \& Arnal M, 1989. Insulin sensitivity and responsiveness during lactation and dry period in goats. American Journal of Physiology 256: E295-E302.

Dechow CD, Rogers GW, \& Clay JS, 2002. Heritability and correlations among body condition score loss, body condition score, production and reproductive performance. $J$ Dairy Sci. 85: 3062-3070. 
DEFRA, 2007. The environmental, social and economic impacts associated with liquid milk consumption in the UK and its production. http: /larchive.defra.gov.uk/foodfarm/food/industry/sectors/milk/pdf/milk-envsoceconimpacts.pdf. Accessed July 10, 2011.

Dieguez C, Carro E, Seoane LM, Garcia M, Camina JP, Senaris R, Popovic V, \& FF Casanueva, 2000. Regulation of somatotroph cell function by the adipose tissue. International Journal of Obesity 24(Suppl. 2): S100-S103.

Dieudonne MN, Bussiere M, Dos Santos E, Leneveu MC, Giudicelli Y, \& Pecquery R. 2006. Adiponectin mediates antiproliferative and apoptotic responses in human MCF7 breast cancer cells. Biochemical Biophysical Research Communication 345: 271-279.

Dobson H, Smith RF, Royal MD, Knight CH, \& Sheldon IM, 2007. The high producing dairy cow and its reproductive performance, Reprod. Domest. Anim. 42(Suppl 2): 17-23.

Domecq JJ, Skidmore AL, Lloyd JW, \& Kaneene JB, 1995. Validation of body condition scores with ultrasound measurements of subcutaneous fat of dairy cows. J Dairy Sci. 78: 2308-2313.

Domecq JJ, Skidmore AL, Lloyd JW, \& Kaneene JB, 1997. Relationship between body condition scores and milk yield in a large dairy herd of high yielding Holstein cows. $J$ Dairy Sci. 80: 101-112.

Downie JG \& Gelman AL, 1976. The relationship between changes in bodyweight plasma glucose and fertility in beef cows. Veterinary Record 99: 210-212.

Downing JA \& Scaramuzzi RJ, 1997. The effect of the infusion of insulin during the luteal phase of the estrous cycle on the ovulation rate and on plasma concentrations of $\mathrm{lh}$, FSH and glucose in ewes. Theriogenology 47: 747-759.

Downing JA, Joss J \& Scaramuzzi RJ, 1999. The effect of a direct arterial infusion of insulin and glucose on the ovarian secretion rates of androstenedione and oestradiol in ewes with an autotransplanted ovary. Journal of Endocrinology 163: 531-541.

Drackley JK, 1999. Biology of dairy cows during the transition period: the final frontier? J Dairy Sci. 82: 2259-2273.

Dridi S \& Taouis M, 2009. Adiponectin and energy homeostasis: consensus and controversy. Journal of Nutritional Biochemistry 20: 831-839.

Duda MK, O'Shea KM, Lei B, Barrows BR, Azimzadeh AM, McElfresh TE, Hoit BD, Kop WJ, \& Stanley WC, 2007. Dietary supplementation with $\omega-3$ PUFA increases adiponectin and attenuates ventricular remodeling and dysfunction with pressure overload. Cardiovascular Research 76: 303-310. 
Dupont J, Chabrolle C, Rame C, Tosca L, \& Coyral-Castel S, 2008. Role of the peroxisome proliferator-activated receptors, adenosinemonophosphate-activated kinase, and adiponectin in the ovary. PPAR Research, pp 1-9.

Easdon MP, Chesworth JM, Aboulela MBE \& Henderson GD, 1985. The effect of undernutrition of beef cows on blood hormone and metabolite concentrations post partum. Reprod. Nutr. Dev. 25: 113-126.

Enders CK. (2010). Applied missing data analysis. In methodology in the Social Sciences (ed.: Kenny DA). $1^{\text {st }}$ edition. Guildford Press. NY. USA.

Engl J, Bobbert T, Ciardi C, Laimer M, Tatarczyk T, Kaser S, Weiss H, Molnar C, Tilg H, Patsch JR, Spranger J, \& Ebenbichler CF, 2007. Effects of pronounced weight loss on adiponectin oligomer composition and metabolic parameters. Obesity 15: 11721175 .

Engström BE, Burman P, Holdstock C \& Karlsson FA, 2003. Effects of Growth Hormone (GH) on Ghrelin, Leptin, and Adiponectin in GH-Deficient Patients. J. Clin. Endocrinol. Metab. 88: 5193-5198.

Etherton TD, 1982. The role of insulin-receptor in interactions in regulation of nutrient utilization by skeletal muscle and adipose tissue: a review. J Anim. Sci. 54: 58-67.

Evans RD, Wallace M, Shalloo L, Garrick DJ, \& Dillon P, 2006. Financial implications of recent declines in reproduction and survival of Holstein- Friesian cows in spring-calving Irish dairy herds, Agricultural Systems 89: 165-183.

Fam BC \& Andrikopoulos S, 2007. The New Zealand obese mouse: polygenic model of obesity, glucose intolerance, and the metabolic syndrome. In animal models of diabetes: frontiers in research (ed. Shafrir E). $2^{\text {nd }}$ Edition, CRC Press, London, UK. pp 139-148.

Fasshauer M, Klein J, Kralisch S, Klier M, Lossner U, Bluher M, \& Paschke R, 2004. Growth hormone is a positive regulator of adiponectin receptor 2 in 3T3-L1 adipocytes. FEBS Letters 28035: 27-32.

Fasshauer M, Klein J, Neumann S, Eszlinger M, \& Paschke R, 2002. Hormonal regulation of adiponectin gene expression in 3T3- L1 adipocytes. Biochem. Biophys. Res. Commun. 290: 1084-1089.

Faulkner DB, Parrett DF, McKeith FK, \& Berger LL, 1990. Prediction of fat cover and carcass composition from live and carcass measurements. Journal of Animal Science 68: $604-610$. 
Field A, 2005. Discovering statistics using SPSS. In ISM introducing statistical methods (ed.: Wright DB). $2^{\text {nd }}$ Edition, SAGE Publications Ltd, London, UK. pp 571-618.

Fouladi-Nashta AA, Gutierrez CG, Garnsworthy PC \& Webb R, 2005. Effects of dietary carbohydrate source on oocyte/embryo quality and development in high-yielding, lactating dairy cattle. Biology of Reproduction (Special issue) 135-136.

Fouladi-Nashta AA, Gutierrez CG, Gong JG, Garnsworthy PC \& Webb R, 2007. Impact of dietary fatty acids on oocyte quality and development in lactating dairy cows, Biology of Reproduction 77: 9-17.

Frees EW 2004. Longitudinal and panel data. Cambridge University Press, UK.

Friggens NC, 2003. Body lipid reserves and the reproductive cycle: towards a better understanding. Livestock Production Science 83: 219-236.

Friggens NC, Andersen JB, Larsen T, Aaes O,\& Dewhurst RJ, 2004. Priming the dairy cow for lactation: a review of dry cow feeding strategies. Anim. Res. 53: 453-473.

Fruhbeck G, Gomez-Ambrosi J, Muruzabal FJ, \& Burrel MA, 2001. The adipocyte: a model for integration of endocrine and metabolic signaling in energy metabolism regulation. Am. J Physiol. Endocrinol. Metab. 280: 827-847.

Gao XL, Yang HX \& Zhao Y 2008. Variations of tumor necrosis factor- $\alpha$, leptin and adiponectin in mid-trimester of gestational diabetes mellitus. Chinese Medical Journal 121(8): 701-705.

Garaulet M, Hernandez-Morante J, Heradia FP, \& Tebar FJ, 2007. Adiponectin, the controversial hormone. Public Health Nutrition: 10(A): 1145-1150.

Garcia MR, Amstalden M, Williams SW, Stanko RL, Morrison CD, Keisler DH, Nizielski SE, \& Williams GL, 2002. Serum leptin and its adipose gene expression during pubertal development, the estrous cycle, and different seasons in cattle. J Anim. Sci. 80: 2158-2167.

Garnsworthy PC, 2006. Body condition score in dairy cows: Targets for production and fertility. In Recent Advances in Animal Nutrition 2006 (eds. PC Garnsworthy \& J Wiseman). University of Nottingham Press. Nottingham. UK. pp 61-86.

Garnsworthy PC \& Jones GP, 1987. The influence of body condition at calving and dietary protein supply on voluntary food intake and performance in dairy cows. Anim. Prod. 44: 347-353. 
Garnsworthy PC \& Topps JH, 1982. The effect of body condition of dairy cows at calving on their food intake and performance when given complete diets. Anim. Prod. 35: 113-119.

Garnsworthy PC \& Webb R, 1999. The Influence of Nutrition on Fertility in Dairy Cows. In Recent advances in animal nutrition - 1999 (ed. Garnsworthy PC \& Wiseman J), Nottingham University Press, Nottingham, UK, pp. 39-57.

Garnsworthy PC, 1988. The effect of energy reserves at calving on performance of dairy cows. In Nutrition and lactation in the dairy cow-1988 (ed. Garnsworthy PC) Proceedings of the 46th University of Nottingham Easter School in Agricultural Science. pp. 157-170.

Garnsworthy PC, Fouladi-Nashta AA, Mann GE, Sinclair KD, \& Webb R, 2009. Effect of dietary-induced changes in plasma insulin concentrations during the early post partum period on pregnancy rate in dairy cows. Reproduction 137: 759-768.

Garnsworthy PC, Lock A, Mann GE, Sinclair KD \& Webb R, 2008b. Nutrition, metabolism and fertility in dairy cows: 1 . Dietary energy source and ovarian function. $J$ Dairy Sci. 91: 3814-3823.

Garnsworthy PC, Lock A, Mann GE, Sinclair KD \& Webb R, 2008c. Nutrition, metabolism, and fertility in dairy cows: 2. Dietary fatty acids and ovarian function. $J$ Dairy Sci. 91: 3824-3833.

Garnsworthy PC, Sinclair KD \& Webb R, 2008a. Integration of physiological mechanisms that influence fertility in dairy cows. Animal 2:(8) 1144-1152.

Gayet C, Leray V, Saito M,\& Siliart B, Nguyen P, 2007. The effects of obesityassociated insulin resistance on mRNA expression of peroxisome proliferatoractivated receptor-g target genes, in dogs. Br. J Nutr. 98: 497-503.

Gearhart MA, Curtis CR, Erb HN, Smith RD, Sniffen CJ, Chase LE, \& Cooper MD, 1990. Relationship of changes in condition score to cow health in Holsteins. J Dairy Sci. 73: $3132-3140$.

Ghanassia E, Brun JF, Mercier J, \& Raynaud E, 2007. Oxidative mechanisms at rest and during exercise. Clinica Chimica Acta 383: 1-20.

Giahi L, Djazayery A, Rahimy A, Rahmany M, \& Larijani B, 2008. Serum level of adiponectin and its association with insulin sensitivity in overweight diabetic and nondiabetic iranian men. Iranian J Publ. Health 37(2): 88-92.

Giannessi D, Maltinti M, Del RS. 2007. Adiponectin circulating levels: A new emerging biomarker of cardiovascular risk. Pharmacological Research 56: 459-467. 
Gillund P, Reksen O, Grohn YT, \& Karlberg K, 2001. Body Condition Related to Ketosis and Reproductive Performance in Norwegian Dairy Cows. J Dairy Sci. 84: 13901396.

Gong JG, Armstrong DG, Baxter G, Hogg CO, Garnsworthy PC and Webb R, 2002a. The effect of increased dietary intake on superovulatory response to FSH in heifers. Theriogenology 57: 1591-1602.

Gong JG, Baxter G, Bramley TA, \& Webb R, 1997. Enhancement of ovarian follicular development in heifers by treatment with recombinant bovine somatotropin: a doseresponse study. Journal of Reproduction \& Fertility 110: 91-97.

Gong JG, Lee WJ, Garnsworthy PC \& Webb R, 2002b. Effect of dietary-induced increases in circulating insulin concentrations during the early postpartum period on reproductive function in dairy cows. Reproduction 123: 419-427.

González CR, Caminos JE, Gallego R, Tovar S, Vázquez MJ, Garcés MF, Lopez M, García-Caballero T, Tena-Sempere $M$, Nogueiras $R, \&$ Diéguez $C, 2010$. Adiponectin receptor 2 is regulated by nutritional status, leptin and pregnancy in a tissue-specific manner. Physiology \& Behavior 99: 91-99.

Goodyear LJ \& Kahn BB, 1998. Exercise, glucose transport, and insulin sensitivity. Annu. Rev. Med. 49: 235-261.

Gordon I, 1996. Controlled Reproduction in Cattle and Buffaloes, CABI Publishing, Wallingford, Oxon, UK.

Gordon ME \& McKeever KH, 2005. Diurnal variation of ghrelin, leptin, and adiponectin in Standardbred mares. J Anim. Sci. 83: 2365-2371.

Gordon ME, McKeever KH, Betros CL, \& Filho HCM, 2007. Exercise-induced alterations in plasma concentrations of ghrelin, adiponectin, leptin, glucose, insulin, and cortisol in horses. The Veterinary Journal 173: 532-540.

Grainger C, Wilhelms GD, \& McGowan AA, 1982. Effect of body condition at calving and level of feeding in early lactation on milk production of dairy cows. Aust. J. Exp. Agric. Anim. Husb. 22: 9-17.

Griinari JM, McGuire MA, Dwyer DA, Bauman DE, Barbano DM, \& House WA, 1996. The role of insulin in the regulation of milk protein synthesis in dairy cows. J Dairy Sci. 80: 2361-2371.

Griinari JM, McGuire MA, Dwyer DA, Bauman DE, Barbano DM, \& House WA, 1997. The role of insulin in the regulation of milk protein synthesis in dairy cows. $J$ Dairy Sci. 80: 2361-2371. 
Grummer RR, 1993. Etiology of lipid-related metabolic disorders in perlparturient dairy cows. J Dairy Sci. 76: 3882-3896.

Grummer RR, 1995. Impact of changes in organic nutrient metabolism on feeding the transition dairy cow. J Anim. Sci. 73: 2820-2833.

Gueorguieva R \& Krystal JH, 2004. Progress in analyzing repeated-measures data and its reflection in papers published in the archives of general psychiatry. Arch Gen Psychiatry 61:310-317.

Guillod-Maximin E, Roy AF, Vacher CM, Aubourg A, Bailleux V, Lorsignol A, Pénicaud L, Parquet M, \& Taouis M, 2009. Adiponectin receptors are expressed in hypothalamus and colocalized with proopiomelanocortin and neuropeptide $\mathrm{Y}$ in rodent arcuate neurons. J Endocrinol. 200: 93-105.

Guo S, 2010. Survival analysis. In pocket guide to social work research methods (ed: Tripodi T), Oxford University Press, Oxford, UK.

Gutierrez CG, Gong JG, Bramley TA, \& Webb R, 1999. Effects of genetic selection for milk yield on metabolic hormones and follicular development in postpartum dairy cattle. $J$ Reprod. Fertil. 24: 16 (Abstract Series 24, Abstract 32).

Gutierrez CG, Gong JG, Bramley TA, \& Webb R, 2006. Selection on predicted breeding value for milk production delays ovulation independently of changes in follicular development, milk production and body weight. Anim. Reprod. Sci. 95: 193-205.

Gutierrez-Aguilar CG, 1997. The effect of nutrition and metabolic hormones on follicular development in cattle. PhD Thesis, Edinburgh University, UK.

Gutman G, Barak V, Maslovitz S, Amit A, Lessing JB, \& Geva E, 2009. Recombinant luteinizing hormone induces increased production of ovarian follicular adiponectin in vivo: implications for enhanced insulin sensitivity. Fertility \& Sterility 91(5): 1837-1842.

Guyton AC \& Hall JE, 2006. Medical Physiology (ed.: Gruliow R), $11^{\text {th }}$ Edition, Philadelphia. Pennsylvania. Saunders Elsevier. pp 829-900.

Hand DJ, 2010. Evaluating diagnostic tests: The area under the ROC curve and the balance of errors. Statist. Med. 29: 1502-1510.

Harden JJ, 2011. A bootstrap method for conducting statistical inference with clustered data. State Politics \& Policy Quarterly 11(2): 223-246.

Harrison RO, Ford SP, Young JW, Conley AJ, \& Freeman AE, 1990. Increased milk production versus reproductive and energy status of high producing dairy cows. $J$ Dairy Sci. 73: 2749-2758. 
Havrankova J, Roth J, \& Brownstein MJ, 1979. Concentrations of insulin and of insulin receptors in the brain are independent of peripheral insulin levels. $J$ Clin. Invest. 64: 636642.

Hayirli A, 2006. The Role of exogenous insulin in the complex of hepatic lipidosis and ketosis associated with insulin resistance phenomenon in postpartum dairy cattle. Veterinary Research Communications 30: 749-774.

Hayirli A, Bertics SJ, \& Grummer RR, 2002. Effects of slow-release insulin on production, liver triglyceride, and metabolic profiles of holsteins in early lactation. $J$ Dairy Sci. 85: 2180-2191.

Herbein JH, Aiello RJ, Eckler LI, Pearson RE, \& Akers RM, 1986. Glucagon, Insulin, growth hormone, and glucose concentrations in blood plasma of lactating dairy cows. $J$ Dairy Sci. 68: 320-325.

Herman MA \& Kahn, BB, 2006. Glucose transport and sensing in the maintenance of glucose homeostasis and metabolic harmony. J Clin. Invest. 116: 1767-1775.

Heuer C, Schukken YH, \& Dobbelaar P, 1999. Postpartum body condition score and results from the first test day milk as predictors of disease, fertility, yield, and culling in commercial dairy herds. J Dairy Sci. 82: 295-304.

Hill RA, 2004. The role of the leptin axis in modulating energy partitioning and nutrient utilisation in livestock species. In Recent advances in animal nutrition (ed. Garnsworthy PC \& Wiseman J). Nottingham University Press, Nottingham, UK. pp 149-184.

Holtenius P \& Holtenius K, 2007. A model to estimate insulin sensitivity in dairy cows. Acta Veterinaria Scandinavica 49:29-32.

Hosmer DW \& Lemeshow S, 2000. Applied logistic regression. In Wiley series in probability and statistics (eds: Cressie NC, Fisher N, Johnstone LM, Kadane JB, Scott DW, Silverman BW, Smith AFM, TeugeIs JL, Barnett V, Bradley RA, Hunter JS, Kendall DG) $2^{\text {nd }}$ Edition, John Wiley and sons Inc, NY, USA.

Hotta K, Funahashi T, Arita Y, Takahashi M, Matsuda M, Okamoto Y, Iwahashi H, Kuriyama H, Ouchi N, Maeda K, Nishida M, Kihara S, Sakai N, Nakajima T, Hasegawa K, Muraguchi M, Ohmoto Y, Nakamura T, Yamashita S, Hanafusa T, \& Matsuzawa Y, 2000. Plasma concentrations of a novel, adipose-specific protein, adiponectin, in type 2 diabetic patients. Arterioscler. Thromb. Vasc. Biol. 20: 1595-1599.

Houghton PL, RP Lemenager, LA Horstman, KS Hendrix, \& G. E. Moss, 1990. Effects of body composition, pre- and postpartum energy level and early weaning on reproductive performance of beef cows and preweaning calf gain. J Anim. Sci. 68: 14381446. 
Houseknecht KL, Baile CA, Matteri RL, \& Spurlock ME, 1998. The biology of leptin: a review, J Anim. Sci. 76: 1405-1420.

Houseknecht KL, Portocarrero CP, Ji S, Lemenager R, \& Spurlock ME, 2000. Growth hormone regulates leptin gene expression in bovine adipose tissue: correlation with adipose IGF-1 expression. Journal of Endocrinology 164: 51-57.

Hull KL \& Harvey S, 2000. Growth hormone: a reproductive endocrine-paracrine regulator? Rev. Reprod. 5: 175-182.

Hull KL \& Harvey S, 2001. Growth hormone: roles in female reproduction. J Endocrinol. 168: $1-23$.

Hull KL \& Harvey S, 2002. GH as a co-gonadotropin: the relevance of correlative changes in GH secretion and reproductive state. J Endocrinol. 172: 1-19.

Huszenicza Gy, Kulcsar M, Nikolic JA, Schmidt J, Korodi P, Katai L, Dieleman S, Ribiczei-Szabo P, \& Rudas P, 2001. Plasma leptin concentration and its interrelation with some blood metabolites, metabolic hormones and the resumption of cyclic ovarian function in postpartum dairy cows supplemented with Monensin or inert fat in feed. In Fertility in the High-Producing Dairy Cow (ed.: Diskin MG), British Society of Animal Science, Edinburgh, pp 405-409.

Ingvartsen KL \& Andersen JB, 2000. Integration of metabolism and intake regulation: a review focusing on periparturient animals. J Dairy Sci. 83: 1573-1597.

Ishioka K, Omachi A, Sagawa M, Shibata H, Honjoh T, Kimura K, \& Saito M, 2006. Canine adiponectin: cDNA structure, mRNA expression in adipose tissues and reduced plasma levels in obesity. Research in Veterinary Science 80: 127-132.

Jackson RA, Wills JR, Kendall NR, Green MJ, Murray RD, \& Dobson H, 2011. Energy metabolites in pre- and postpartum dairy cattle as predictors of reproductive disorders. Veterinary Record 168: 562-571.

Jarde T, Caldefie-Chezet F, Goncalves-Mendes N, Mishellany F, Buechler C, PenaultLlorca F, \& Vasson MP, 2009. Involvement of adiponectin and leptin in breast cancer: clinical and in vitro studies. Endocrine-Related Cancer 16: 1197-1210.

Jensen JR \& Alvero R, 2007. Polycystic ovary syndrome. In reproductive endocrinology and infertility (eds: Alvero R \& Schlaff WD), Mosby Elsevier Inc, Philadelphia, USA. pp 65-76.

Jiang G \& Zhang BB, 2003. Glucagon and regulation of glucose metabolism. Endocrinol. Metab. 284: 671-678. 
Jones HN, Woollett LA, Barbour N, Prasad PD, Powell TL, \& Jansson T, 2009. Highfat diet before and during pregnancy causes marked up-regulation of placental nutrient transport and fetal overgrowth in C57/BL6 mice. The FASEB Journal 23: 271-278.

Jorritsma R, Wensing T, Kruip TAM, Vos PLAM, Noordhuizen JPTM 2003. Metabolic changes in early lactation and impaired reproductive performance in dairy cows. Vet. Res. 34: 11-26.

Kadokawa H, Blache D, Yamada Y, \& Martin GB, 2000 .Relationships between changes in plasma concentrations of leptin before and after parturition and the timing of first post-partum ovulation in high-producing Holstein dairy cows. Reproduction, Fertility, and Development 12: 405-411.

Kadowaki T \& Yamauchi T, 2005. Adiponectin and adiponectin receptors. Endocrine Reviews 26(3): 439-451.

Kadowaki T, Yamauchi T, \& Kubota N, 2008. The physiological and pathophysiological role of adiponectin and adiponectin receptors in the peripheral tissues and CNS. FEBS Letters 582: 74-80.

Kadowaki T, Yamauchi T, Kubota N, Hara K, Ueki K, \& Tobe K, 2006. Adiponectin and adiponectin receptors in insulin resistance, diabetes, and the metabolic syndrome. $J$. Clin. Invest. 116: 1784-1792.

Kadzere CT, Murphy MR, Silanikove N \& Maltz E, 2002. Heat stress in lactating dairy cows: a review. Livestock Prod. Sci. 77: 59-91.

Kalro BN, 2003. Impaired fertility caused by endocrine dysfunction in women. Endocrinol. Metab. Clin. N Am. 32: 573-592.

Kamada Y, Takehara T, \& Hayashi N, 2008. Adipocytokines and liver disease. $J$ Gastroenterol 43: 811-822.

Kaneko JJ, 2008a. Carbohydrate metabolism and its diseases. In Clinical Biochemistry of Domestic Animals, $6^{\text {th }}$ Edition (eds: Kaneko JJ, Harvey HW \& Bruss ML). Academic Press, Elsevier Inc, San Diego, CL, USA, pp 45-80.

Kaneko JJ, 2008b. Appendix VIII blood analyte reference values in large animals. In Clinical Biochemistry of Domestic Animals, $6^{\text {th }}$ Edition (eds: Kaneko JJ, Harvey HW \& Bruss ML). Academic Press, Elsevier Inc, San Diego, CL, USA, pp 882-888.

Kawashima C, Fukihara S, Maeda M, Kaneko E, Montoya CA, Matsui M, Shimizu T, Matsunaga N, Kida K, Miyake YI, Schams D, \& Miyamoto A, 2007. Relationship between metabolic hormones and ovulation of dominant follicle during the first follicular wave post-partum in high-producing dairy cows. Reproduction 133: 155-163. 
Kearns CF, McKeever KH, Roegner V, Brady SM, \& Malinowski K, 2006. Adiponectin and leptin are related to fat mass in horses. Vet $J$ 172: 460-465.

Kern PA, Di Gregorio GB, Lu T, Rassouli N, \& Ranganathan G, 2003. Adiponectin expression from human adipose tissue: relation to obesity, insulin resistance, and tumor necrosis factor-alpha expression. Diabetes 52: 1779-1785.

Kershaw E \& Flier J, 2004. Adipose tissue as an endocrine organ. $J$ Clin. Endocrinol. Metab. 89: 2548-2556.

Kharroubi I, Rasschaert J, Eizirik DL, \& Cnop M, 2003. Expression of adiponectin receptors in pancreatic $\beta$ cells. Biochem. Biophys. Res. Commun. 312: 1118-1122.

Kido Y, Nakae J, \& Accili D, 2001. The insulin receptor and its cellular targets. The Journal of Clinical Endocrinology \& Metabolism 86: 972-979.

Klein JP \& Moeschberger ML, 2003. Survival analysis techniques for censored and truncated data. In Statistics for Biology \& Health (eds: Dietz K, Gail M, Krickeberg K, Samet J, Tsiatis A). Springer-Verlag, New York, Inc.

Klip A, Tsakiridis T, Marette A, \& Ortiz PA, 1994. Regulation of expression of glucose transporters by glucose: a review of studies in vivo and in cell cultures. The FASEB Journal 8: 43-53.

Kokta TA, Dodson MV, Gertler A, \& Hill RA, 2004. Intracellular signaling between adipose tissue and muscle tissue. Domestic Animal Endocrinology 27: 303-331.

Komatsu T, Itoh F, Sakumoto R, Hodate K, Obara Y \& Kushibiki S, 2007. Changes in the gene expression of adiponectin and glucose transporter 12 (GLUT12) in lactating and non-lactating cows. Animal Science Journal 78: 98-102.

Krasnow SM \& Steiner RA, 2006. Physiological mechanisms integrating metabolism and reproductionin. In Knobil and Neill's Physiology of Reproduction. (ed: Neill JD) 3rd edition, New York, Elsevier, pp 2553-2625.

Krentz AJ, 1996. Insulin Resistance. BMJ 331:1385-1389.

Kronfeld DS, Mayer GP, Robertson JMD, \& Raggi F , 1963. Depression of milk secretion during insulin administration. J Dairy Sci. 46: 559-563.

Kubota N, Yano W, Kubota T, Yamauchi T, Itoh S, Kumagai H, Kozono H, Takamoto I, Okamoto S, Shiuchi T, 2007. Adiponectin stimulates AMP- activated protein kinase in the hypothalamus and increases food intake. Cell Metab. 6: 55-68. 
Laarveld B, Chaplin RK, \& Brockman RP 1985. Effects of insulin on the metabolism of acetate, $\beta$-hydroxybutyrate, and triglycerides by the bovine mammary gland. Comparative Biochemistry \& Physiology 82: 265-267.

Lagaly DV, Aad PY, Grado-Ahuir JA, Hulsey LB, \& Spicer LJ, 2008. Role of adiponectin in regulating ovarian theca and granulosa cell function. Molecular and Cellular Endocrinology 284: 38-45.

Lake SL, Scholljegerdes EJ, Atkinson RL, Nayigihugu V, Paisley SI, Rule DC, Moss GE, Robinson TJ, \& Hess BW, 2005. Body condition score at parturition and postpartum supplemental fat effects on cow and calf performance. J Anim. Sci. 83: 2908-2917.

Lake SL, Scholljegerdes EJ, Hallford DM, Moss GE, Rule DC, \& Hess BW, 2006. Effects of body condition score at parturition and postpartum supplemental fat on metabolite and hormone concentrations of beef cows and their suckling calves. $J$ Anim. Sci. 84: 1038-1047.

Lam KSL, Xu A, Tan KCB, Wong LC, Tiu SC, \& Tam S, 2004. Serum adiponectin is reduced in acromegaly and normalized after correction of growth hormone excess. $J$ Clin. Endocrinol. Metab. 89: 5448-5453.

Landau S \& Everitt BS, 2004. A handbook of statistical analyses using SPSS. Chapman \& Hall/CRC Press LLC, London, UK.

Langhans W \& Geary N, 2010. Overview of the physiological control of eating. In Frontiers in eating and weight regulation (eds: Langhans $\mathrm{W} \&$ Geary N). Forum Nutr. Basel, Karger, vol 63, pp 9-53.

Ledoux S, Campos DB, Lopes FL, Dobias-Goff M, Palin MF, \& Murphy BD, 2006. Adiponectin induces periovulatory changes in ovarian follicular cells. Endocrinology 147: 5178-5186.

Ledoux D, Touze JL, Richard C, Ponter AA, Bosc MJ, \& Grimard B, 2011. Abnormal patterns of resumption of cyclicity after calving in Holstein cows: risk factors, relationships with the ultrasound appearance of the ovaries and with gestation failure after AI. Revue Méd. Vét. 162(2): 98-106.

Lely AT, Krikken JA, Bakker SJL, Boomsma F, Dullaart RPF, Wolffenbuttel BHR, \& Navis G, 2007. Low dietary sodium and exogenous angiotensin II infusion decrease plasma adiponectin concentrations in healthy men. J. Clin. Endocrinol. Metab. 92: 18211826.

Lemor A, Mielenz M, Altmann M, von Borell E, \& Sauerwein H, 2010. mRNA abundance of adiponectin and its receptors, leptin and visfatin and of G-protein coupled 
receptor 41 in five different fat depots from sheep. Journal of Animal Physiology \& Animal Nutrition 94: E96-E101.

Lents CA, White FJ, Ciccioli NH, Wettemann RP, Spicer LJ \& Lalman DL, 2008. Effects of body condition score at parturition and postpartum protein supplementation on estrous behavior and size of the dominant follicle in beef cows. J Anim. Sci. 86: 25492556.

León HV, Hernández-Cerón J, Keisler DH, \& Gutierrez CG, 2004. Plasma concentrations of leptin, insulin-like growth factor-I, and insulin in relation to changes in body condition score in heifers. J. Anim. Sci. 82:445-451.

Leroy JLMR, Langbeen A, Van Hoeck V, Bols PEJ, 2010. Metabolism and reproduction, the battle for nutrients. Advances in Dairy Technology 22: 25-34.

Leroy JLMR, Vanholder T, Van Knegsel ATM, Garcia-Ispierto I \& Bols PEJ, 2008. Nutrient Prioritization in dairy cows early postpartum: mismatch between metabolism and fertility? Reprod. Dom. Anim. 43 (Suppl. 2): 96-103.

Leury BJ, Baumgard LH, Block SS, Segoale N, Ehrhardt RA, Rhoads RP, Bauman DE, Bell AW, \& Boisclair YR, 2003. Effect of insulin and growth hormone on plasma leptin in periparturient dairy cows. Am. J Physiol. Regul. Integr. Comp. Physiol. 285: 1107-1115.

Lewis D \& Hill KJ 1983. The provision of nutrients. In Nutritional Physiology of Farm Animals (ed.: Rook JAF \& Thomas PC), Longman, UK, pp 3-40.

Liefers SC, Veerkamp RF, Te Pas MFW, Chilliard Y, \& Van der Lende T, 2005. Genetics and physiology of leptin in periparturient dairy cows. Domestic Animal Endocrinology 29: 227-238.

Little RJA \& Rubin DB, 1987. Statistical analysis with missing data. John Wiley \& Sons, NY, USA.

Littell RC, Henry PR \& Ammerman CB, 1998. Statistical analysis of repeated measures data using SAS procedures. J Anim Sci 76:1216-1231.

Littell RC, 2002. Analysis of unbalanced mixed model data: A case study comparison of anova versus REML/GLS. Journal of Agricultural, Biological, and Environmental Statistics 7(4): 472-490.

Liu GW, Zhang ZG, Zhu XL, Wang Z, Xia C \& Xu C, 2009. A note on the distribution of insulin receptor mRNA in tissues of neonatal Holstein calves. Journal of Animal \& Feed Sciences 18: 613-619. 
Liu YH, Tsai EM, Chen YL, Chen HS, Chen YC, Wu LC, Lee CH, Jong SB, \& Chan TF, 2006. Serum adiponectin levels increase after human chorionic gonadotropin treatment during in vitro fertilization. Gynecol. Obstet. Invest. 62: 61-65.

Liu H \& Li G, 2005. Testing statistical significance of the area under a receiving operating characteristics curve for repeated measures design with bootstrapping. Journal of Data Science 3: 257-278.

Lopez-Gatius, Yaniz FJ, \& Madriles- Helm D, 2003. Effects of body condition score and score change on the reproductive performance of dairy cows: A meta-analysis. Theriogenology 59: 801-812.

Lord E, Ledoux S, Murphy BD, Beaudry D, \& Palin MF, 2005. Expression of adiponectin and its receptors in swine. J Anim. Sci. 83: 565-578.

Lucy MC, 2000. Regulation of ovarian follicular growth by somatotropin and insulin-like growth factors in cattle. J Dairy Sci. 83: 1635-1647.

Lucy MC, 2001. Reproductive loss in high-producing dairy cattle: Where Will It End? $J$ Dairy Sci. 84: 1277-1293.

Lucy MC, 2002. The future of dairy reproductive management, Advances in Dairy Technology 14: 161-173.

Lucy MC, 2008. Functional differences in the growth hormone and insulin-like growth factor axis in cattle and pigs: implications for post-partum nutrition and reproduction. Reprod. Dom. Anim. 43 (Suppl. 2): 31-39.

Lukes AJ, Barnes MA, \& Pearson RE, 1989. Response to selection for milk yield and metabolic challenges in primiparous dairy cows. Domest. Anim. Endocrinol. 6: 287-298.

Macajova M, Lamosova D, \& Zeman M, 2004. Role of leptin in farm animals: a review. J Vet. Med. A51: 157-166.

Mackle TR, Dwyer DA, \& Bauman DE, 1999. Effects of branched-chain amino acids and sodium caseinate on milk protein concentration and yield from dairy cows. $J$ Dairy Sci. 82: 161-171.

Mackle TR, Dwyer DA, \& Bauman DE, 2000. Intramammary infusion of insulin or long r3 insulin-like growth factor-i did not increase milk protein yield in dairy cows. $J$ Dairy Sci. 83: $1740-1749$.

Macmillan KL, Lean IJ, \& Westwood CT, 1996. The effects of lactation on the fertility of dairy cows. Aust. Vet. J 73: 141-147. 
Maillard V, Uzbekova S, Guignot F, Perreau C, Ramé C, Coyral-Castel S, Dupont J, 2010. Effect of adiponectin on bovine granulosa cell steroidogenesis, oocyte maturation and embryo development. Reproductive Biology \& Endocrinology 8:23.

Marieb EN \& Hoehn K, 2007. Human anatomy and physiology (ed.: Cummings B), $7^{\text {th }}$ Edition, Pearson Education Inc.

Martin LJ, Woo JG, Geraghty SR, Altaye M, Davidson BS, Banach W, Dolan LM, Ruiz-Palacios GM, \& Morrow AL, 2006. Adiponectin is present in human milk and is associated with maternal factors. Am. J Clin. Nutr. 83:1106-1111.

Mashek DG, Ingvartsen KL, Andersen JB, Vestergaard M, \& Larsen T, 2001. Effects of a four-day hyperinsulinemic-euglycemic clamp in early and mid-lactation dairy cows on plasma concentrations of metabolites, hormones, and binding proteins. Domestic Animal Endocrinology 21:169-185.

Masters BA, Shemer J, Judkins JH, Clarke DW, Le Roith D, \& Raizada MK, 1987. Insulin receptors and insulin action in dissociated brain cells. Brain Research 417: 247 256.

Matsuzawa Y, Funahashi T, Kihara S,\& Shimomura I, 2004. Adiponectin and metabolic syndrome. Arterioscler. Thromb. Vasc. Biol. 24:29-33.

Mauras N \& Haymond MW, 2005. Are the metabolic effects of GH and IGF-I separable? Growth Hormone \& IGF Research 15:19-27.

Myers MG, Cowley MA, \& Munzberg H, 2008. Mechanisms of leptin action and leptin resistance. Annu. Rev. Physiol. 70:537-56.

Mazaki-Tovi S, Kanety H, \& Sivan E, 2005. Adiponectin and human pregnancy. Current Diabetes Reports 5:278-281.

McClure TJ, 1967. Infertility in mice caused by fasting at about the time of mating. $J$. Reprod. Fert. 13:393-403.

McGuire MA, Bauman DE, Dwyer DA, \& Cohick WS, 1995. Nutritional modulation of the somatotropin/insulin-like growth factor system: response to feed deprivation in lactating cows. $J$ Nutr. 125: 493-502.

McGuire MA, Dwyer DA, Bauman DE, \& Smith DF, 1998. Insulin-like growth factors in plasma and afferent mammary lymph of lactating cows deprived of feed or treated with bovine somatotropin. J Dairy Sci. 81: 950-987.

McGuire MA, Griinari JM, Dwyer DA, \& Bauman DE, 1995. Role of insulin in the regulation of mammary synthesis of fat and protein. J Dairy Sci. 78:816-824. 
McGuire MA, Vicini JL, Bauman DE \& Veenhuizen JJ, 1992. Insulin-like growth factors and binding proteins in ruminants and their nutritional regulation. J Anim. Sci. 70: 2901-2910.

Michalakis KG \& Segars JH, 2010. The role of adiponectin in reproduction: from polycystic ovary syndrome to assisted reproduction. Fertility \& Sterility 94(6):1949-1957.

Miller DW, Keller BT, \& Borchardt RT, 1994. Identification and distribution of insulin receptors on cultured bovine brain microvessel endothelial cells: possible function in insulin processing in the blood-brain barrier. Journal of Cellular Physiology 161: 333-341.

Minke A, 1997. Conducting repeated measures analyses: Experimental design considerations. Download from http://ericae.net/ft/tamu/Rm.htm. Accessed August 11, 2011.

Minokoshi Y, Shiuchi T, Lee S, Suzuki A, \& Okamoto S, 2008. Role of hypothalamic AMP-kinase in food intake regulation. Nutrition 24: 786-790.

Mitchell M, Armstrong DT, Robker RL, \& Norman RJ, 2005. Adipokines: implications for female fertility and obesity. Reproduction 130: 583-597.

Mitev J, Miteva T, Stoyanchev T, \& Binev R, 2005. Welfare and health of dairy cattle effect of the level of nutrition and body condition at the end of pregnancy and early lactation. Trakia Journal of Sciences 3(2): 78-86.

Miyoshi S, Pate JL, Palmquist DL, 2001. Effects of propylene glycol drenching on energy balance, plasma glucose, plasma insulin, ovarian function and conception in dairy cows. Animal Reproduction Science 68: 29-43.

Moran C, Quirke JF, \& Roche JF, 1989. Puberty in heifers: a review. Animal Reproduction Science 18: 167-182.

Moran TH, 2010. Hypothalamic nutrient sensing. In frontiers in eating and weight regulation (eds: Langhans W \& Geary N). Forum Nutr. Basel, Karger, vol 63, pp 94-101.

Moreira F, Risco C, Pires MFA, Ambrose JD, Drost M, DeLorenzo M, \& Thatcher WW, 2000. Effect of body condition on reproductive efficiency of lactating dairy cows receiving a timed insemination. Theriogenology 53: 1305-1319.

Mullen KL, Smith AC, Junkin KA, \& Dyck DJ, 2007. Globular adiponectin resistance develops independently of impaired insulin-stimulated glucose transport in soleus muscle from high-fat-fed rats. Am. J Physiol. Endocrinol. Metab. 293: E83-E90. 
Mulligan F, O'Grady L, Rice D \& Doherty M, 2006a. A herd health approach to dairy cow nutrition and production diseases of the transition cow. Animal Reproduction Science 96: $331-353$.

Mulligan F, O'Grady L, Rice D \& Doherty M, 2006b. Production diseases of the transition cow: Body condition score and energy balance . Irish Veterinary Journal 59(9): 505-510.

Nafikov RA \& Beitz DC, 2007. Carbohydrate and lipid metabolism in farm animals. $J$ Nutr. 137: 702-705.

Nafikov RA, Ametaj BN, Bobe G, Koehler KJ, Young JW, \& Beitz DC, 2006. Prevention of fatty liver in transition dairy cows by subcutaneous injections of glucagon. $J$ Dairy Sci. 89: 1533-1545.

Nagatani S, Zeng Y, Keisler DH, Foster DL \& Jaffe CA, 2000. Leptin regulates pulsatile luteinizing hormone and growth hormone secretion in sheep. Endocrinology 141: 3965-3975.

Nebel RL \& McGllliard ML, 1998. Interactions of high milk yield and reproductive performance in dairy cows. J Dairy Sci. 76: 3257-3268.

Neville MC, 2006. Lactation and its hormonal control. In Knobil and Neill's Physiology of Reproduction. (ed.: Neill JD) $3^{\text {rd }}$ edition, New York, Elsevier, pp 2993-3054.

Newburg DS, Woo JG, \& Morrow AL, 2010. Characteristics and potential functions of human milk adiponectin. J Pediatr. 156(2 Suppl): S41-S46.

Nielsen MO, Madsen TG, \& Hedeboe AM, 2001. Regulation of mammary glucose uptake in goats: role of mammary gland supply, insulin, IGF-1 and synthetic capacity. Journal of Dairy Research 68: 337-349.

Nien JK, Mazaki-Tovi S, Romero R, Erez O, Kusanovic JP \& Gotsch F, 2007. Plasma adiponectin concentrations in non pregnant, normal pregnancy and overweight pregnant women. J Perinat. Med 35(6): 522-531.

Nieuwenhuizen AG, Schuiling GA, Bonen A, Paans AM, Waalburg W, \& Koiter, TR, 1998. Glucose consumption by various tissues in pregnant rats: effects of a 6-day euglycaemic hyperinsulinaemic clamp. Acta Physiologica Scandinavica, 164: 325-334.

Nieuwenhuizen AG, Schuiling GA, Moes H, \& Koiter TR, 1997. Role of increased insulin demand in the adaptation of the endocrine pancreas to pregnancy. Acta Physiologica Scandinavica 159: 302-312. 
Nilsson L, Binart N, Bohlooly-Y M, Bramnert M, Egecioglu E, Kindblom J, Kelly PA, Kopchick JJ, Ormandy CJ, Ling C, \& Billig H, 2005. Prolactin and growth hormone regulate adiponectin secretion and receptor expression in adipose tissue. Biochemical \& Biophysical Research Communications 331: 1120-1126.

Nishimoto H, Matsutani R, Yamamoto S, Takahashi T, Hayashi KG, Miyamoto A, Hamano S \& Tetsuka M, 2006. Gene expression of glucose transporter (GLUT) 1, 3 and 4 in bovine follicle and corpus luteum. Journal of Endocrinology 188: 111-119.

Norman JE, 2010. The adverse effects of obesity on reproduction. Reproduction 140: 343345.

Ohtani Y, Yonezawa T, Song SH, Takahashi T, Ardiyanti A, Sato K, Hagino A, Roh SG, \& Katoh K 2011. Gene expression and hormonal regulation of adiponectin and its receptors in bovine mammary gland and mammary epithelial cells. Animal Science Journal 82: 99-106.

Oltenacu PA \& Algers B, 2005. Selection for increased production and the welfare of dairy cows: are new breeding goals needed? Royal Swedish Academy of Sciences, Ambio Vol. 34: 311-315.

Opsomer G, Grohn YT, Hertl J, Coryn M, Deluyker H, \& de Kruif A, 2000. Risk factors for post partum ovarian dysfunction in high producing dairy cows in belgium: a field study. Theriogenology 53: 841-857.

Opsomer G, Leroy J, Vanholder T, Bossaert P, \& de Kruif A, 2006. Optimizing dairy cow reproductive performances: Besides the use of hormones. World Buiatrics Congress 2006 - Nice, France.

Osman MA, Allen PS, Bobe G, Coetzee JF, Abuzaid A, Koehler K, \& Beitz DC, 2010. Chronic metabolic responses of postpartal dairy cows to subcutaneous glucagon injections, oral glycerol, or both. J Dairy Sci. 93: 3505-3512.

Osman MA, Allen PS, Mehyar NA, Bobe G, Coetzee JF, Koehler KJ, \& Beitz DC, 2008. Acute metabolic responses of postpartal dairy cows to subcutaneous glucagon injections, oral glycerol, or both. J Dairy Sci. 91: 3311-3322.

Page ST, Herbst KL, Amory JK, Coviello AD, Anawalt BD, Matsumoto AM, \& Bremner WJ, 2005. Testosterone administration suppresses adiponectin levels in men. $J$ Androl. 26: 85-92.

Pasquali R \& Gambineri A, 2006. Metabolic effects of obesity on reproduction. Reproductive BioMedicine 12(5): 542-551.

PASW $^{\circledR} 18$ User's Guide: Advanced Statistics, 2009, SPSS Inc, USA. 
Paula-Lopes FF, Boelhauve M, Habermann FA, Sinowatz F, \& Wolf E, 2007. Leptin promotes meiotic progression and developmental capacity of bovine oocytes via cumulus cell-independent and -dependent mechanisms. Biology of Reproduction 76: 532-541.

Pedron O, Cheli F, Senatore E, Baroli D,\& Rizzi R, 1993. Effect of body condition score at calving on performance, some blood parameters, and milk fatty acid composition in dairy cows. $J$ Dairy Sci. 76: 2528-2535.

Pehrson B, Forshell KP, \& Carlsson J, 1992. The effect of additional feeding on the fertility of high-yielding dairy cows. Journal of Veterinary Medicine Series 39(A):187192.

Peng CYJ, Lee KL, \& Ingersoll GM, 2002. An introduction to logistic regression analysis and reporting. $J$ Educ. Research 96(1): 3-14.

Petitclerc D, Lacasse P, Girard CL, Boettcher PJ, \& Block E, 2000. Genetic, nutritional and endocrine support of milk synthesis in dairy cows. J Anim. Sci. 78 (Suppl. 3): 59-77.

Pierre P, Froment P, Negre D, Rame' C, Barateau V, Chabrolle C, Lecomte P, \& Dupont J, 2009. Role of adiponectin receptors, AdipoR1and AdipoR2, in the steroidogenesis of the human granulosa tumor cell line, KGN. Human Reproduction $\mathbf{0}(0)$ : $1-12$.

Pischon T, Girman CJ, Rifai N, Hotamisligil GS, \& Rimm EB, 2005. Association between dietary factors and plasma adiponectin concentrations in men. Am. J Clin. Nutr. 81: 780-786.

Poretsky L, Cataldo NA, \& Rosenwaks Z, 1999. The insulin-related ovarian regulatory system in health and disease. Endocrine Reviews 20: 535-582.

Popovic V, Damjanovic S, Dieguez C, \& Casanueva FF, 2001. Leptin and the pituitary. Pituitary 4: 7-14.

Pratt SE, Geor RJ, \& McCutcheon LJ, 2005. Relationship of insulin sensitivity to aerobic capacity in standardbred mares and geldings. Eq. Comp. Exerc. Physiol. 2: 185193.

Prior RL \& Christenson RK, 1978. Insulin and glucose effects on glucose metabolism in pregnant and nonpregnant ewes. J Anim. Sci. 46: 201-210.

Pryce JE, \& Harris BL, 2006. Genetics of body condition score in New Zealand dairy cows. J Dairy Sci. 89: 4424-4432.

Pryce JE, Coffey MP, \& Simm G, 2001. The relationship between body condition score and reproductive performance. J Dairy Sci. 84: 1508-1515. 
Pryce JE, Nielsen BL, Veerkamp RF, \& Simm G, 1999. Genotype and feeding system effects and interactions for health and fertility traits in dairy cattle. Livest. Prod. Sci. 57: 193-201.

Puntenney SB, 2006. The effect of prepartum anionic diets on cortisol, adiponectin, and tumour necrosis factor- $\alpha$ expression at varying levels of body mass index in preparturient dairy cows: implications for insulin resistance. PhD Thesis. Oregon State University. USA.

Qi L, Rimm E, Liu S, Rifai N, \& Hu FB, 2005. Dietary glycemic index, glycemic load, cereal fiber, and plasma adiponectin concentration in diabetic men. Diabetes Care 28(5): 1022-1028.

Qi Y, Takahashi N, Hileman SM, Patel HR, \& Berg AH, 2004. Adiponectin acts in the brain to decrease body weight. Nat. Med. 10: 524-529.

Rabiee AR, Lean IJ, Gooden JM, \& Miller BG, 1999. Relationships among metabolites influencing ovarian function in the dairy cow. J Dairy Sci. 82: 39-44.

Raddatz JR, Elias AN, \& Whisnant CS, 2008. Measurements of Adiponectin in lactating dairy cows. J Anim. Sci.: 86 (E-Suppl.2):60.

Rajaram S, Baylink DJ, \& Mohan S, 1997. Insulin-like growth factor-binding proteins in serum and other biological fluids: regulation and functions. Endocr. Rev. 18: 801-831.

Ramachandran R, Ocon-Grove OM, \& Metzger SL, 2007. Molecular cloning and tissue expression of chicken AdipoR1 and AdipoR2 complementary deoxyribonucleic acids. Domestic Animal Endocrinology 33: 19-31.

Ramsay JE, Greer I, \& Sattar N, 2006. Obesity and reproduction. BMJ 333: 1159-1162.

Rechler MM \& Nissley SP, 1985. The nature and regulation of the receptors for insulinlike growth factors. Ann. Rev. Physiol. 47: 425-442.

Reis CEG, Bressan J, \& Alfenas RCG, 2010. Effect of the diet components on adiponectin levels. Nutr. Hosp. 25 (6): 881-888.

Reinehr T, Roth C, Menke T, \& Andler W, 2004. Adiponectin before and after weight loss in obese children. J Clin. Endocr. \& Metab. 89(8): 3790-3794.

Remppis S, Steingass H, Gruber L, \& Schenkel H, 2011. Effects of energy intake on performance, mobilization and retention of body tissue, and metabolic parameters in dairy cows with special regard to effects of pre-partum nutrition on lactation. Asian-Aust. $J$ Anim. Sci. 24(4): 540- 572. 
Reynolds CK, 2005. Glucose balance in cattle. Florida Ruminant Nutrition Symposium. pp 143-154.

Rhoads RP, Kim JW, Leury BJ, Baumgard LH, Segoale N, Frank SJ, Bauman DE, \& Boisclair YR 2004. Insulin increases the abundance of the growth hormone receptor in liver and adipose tissue of periparturient dairy cows. J. Nutr. 134(5): 1020-1027.

Ritterath C, Rad NT, Siegmund T, Heinze T, Siebert G, Buhling KJ, 2010. Adiponectin during pregnancy: correlation with fat metabolism, but not with carbohydrate metabolism. Arch. Gynecol. Obstet. 281: 91-96.

Roberts AJ, Nugent RA, Klint J, \& Jenkins TG, 1997. Circulating insulin-like growth factor binding proteins, growth hormone, and resumption of estrus in postpartum cows subjected to dietary energy restriction. J Anim. Sci. 75: 1909-1917.

Robinson JJ, Ashworth CJ, Rooke JA, Mitchell LM, \& McEvoy TG, 2006. Nutrition and fertility in ruminant livestock. Animal Feed Science and Technology 126: 259-276.

Roche JR, Lee JM, Macdonald KA, \& Berry DP, 2007a. Relationships among body condition score, body weight, and milk production variables in pasture-based dairy cows. $J$ Dairy Sci. 90: 3802-3815.

Roche JR, Friggens NC, Kay JK, Fisher MW, Stafford KJ, \& Berry DP, 2009. Invited review: Body condition score and its association with dairy cow productivity, health, and welfare. J Dairy Sci. 92: 5769-5801.

Roche JR, Macdonald KA, Burke CR, Lee JM, \& Berry DP, 2007b. Associations among body condition score, body weight, and reproductive performance in seasonalcalving dairy cattle. J Dairy Sci. 90: 376-391.

Roden M \& Bernroider E, 2003. Hepatic glucose metabolism in humans- its role in health and disease. Best Practice \& Research Clinical Endocrinology \& Metabolism 17(3): 365-383.

Rodriguez-Pacheco F, Martinez-Fuentes AJ, Tovar S, Pinilla L, Tena-Sempere M, Dieguez C, Castaño JP, \& Malagon MM, 2007. Regulation of pituitary cell function by adiponectin. Endocrinology 148: 401-410.

Ronge H, Blum JW, Clemment C, Jans F, Leuenbeger H, \& Binder H, 1988. Somatomedin $\mathrm{C}$ in dairy cows related to energy and protein supply and to milk production. Anim. Prod. 47: 165-183.

Ronti T, Lupattelli G, \& Mannarino E, 2006. The endocrine function of adipose tissue: an update. Clin. Endocrinol. 64: 355-65. 
Rook JAF \& Thomas PC, 1983. Milk Production. In Nutritional Physiology of Farm Animals (ed: Rook JAF \& Thomas PC), Longman. UK. pp 558-607.

Rose MT, Obara Y, Itoh F, Hashimoto H \& Takahashi Y, 1997. Growth hormone does not affect non-insulin-mediated glucose uptake in sheep. Experimental Physiology 82: 749760 .

Rosen CJ \& Pollak M, 1999. Circulating IGF-I. New perspectives for a new century. Trends Endocrinol. Metab. 10: 136-141.

Royal MD, Darwash AO, Flint APF, Webb R, Woolliams JA, \& Lamming GE, 2000. Declining fertility in dairy cattle: changes in traditional and endocrine parameters of fertility. Animal Science 70: 487-501.

Ruan H, Miles PD, Ladd CM, Ross K, Golub TR, \& Olefsky JM, 2002. Profiling gene transcription in vivo reveals adipose tissue as an immediate target of tumor necrosis factoralpha: implications for insulin resistance. Diabetes 51: 3176-3188.

Rudolph MC, Neville MC, \& Anderson SM, 2007. Lipid synthesis in lactation: diet and the fatty acid switch. J Mammary Gland Biol Neoplasia 12: 269-281.

Ruegg PL, Goodger WJ, Holmberg CA, Weaver LD, \& Huffman EM, 1992. Relation among body condition score, serum urea nitrogen and cholesterol concentrations, and reproductive performance in high-producing Holstein dairy cows in early lactation. Am. $J$ Vet. Res. 53: 10-14.

Ruegg PL \& Milton RL, 1995. Body condition scores of Holstein cows on Prince Edward Island, Canada: Relationships with yield, reproductive performance, and disease. J Dairy Sci. 78: 552-564.

Ryan DP, Spoon RA, Griffith MK, \& Williams GL, 1994. Ovarian follicular recruitment, granulosa cell steroidogenic potential, and growth hormone/insulin-like growth factor-1 relationships in beef cows consuming high lipid diets: Effects of graded differences in body condition maintained during the puerperium. Dom. Anim. Endocrinol. 11: 161-174.

Sakai T, Hayakawa T, Hamakawa M, Ogura K \& Kubo S, 1993. Therapeutic effects of simultaneous use of glucose and insulin in ketotic dairy cows. J Dairy Sci. 76: 109-114.

Sano H, Nakai M, Kondo T, \& Terashima Y, 1991. Insulin responsiveness to glucose and tissue responsiveness to insulin in lactating, pregnant, and nonpregnant, nonlactating beef cows. J Anim. Sci. 69: 1122-1127. 
Sano H, Narahara S, Kondo T, Takahashi A \& Terashima Y, 1993. Insulin responsiveness to glucose and tissue responsiveness to insulin during lactation in dairy cows. Dom. Anim. Endocrinol. 10: 191-197.

Santos JEP, 2007. Impact of nutrition on dairy cattle reproduction. Mid-South Ruminant Nutrition. Arlington, Texas. pp 35-48.

Santos JEP, 2009. Nutrition and reproduction in dairy cattle. Dairy Caltle Reproduction Conference. Minneapolis, MN, Boise. pp 93-102.

Santos JEP, Bisinotto RS, Ribeiro ES, Lima FS, Greco LF, Staples CR, \& Thatcher WW, 2010. Applying nutrition and physiology to improve reproduction in dairy cattle. In Reproduction in Domestic Ruminants VII (eds: Lucy MC, Pate JL, Smith MF \& Spencer TE). Society of reproduction and fertility volume 67, Proceedings of the eighth international symposium on reproduction in domestic ruminants. Anchorage, Alaska. Nottingham University Press, Nottingham, UK, pp 387-404.

Sasaki S, 2002. Mechanism of insulin action on glucose metabolism in ruminants. Animal Science Journal 73: 423-433.

Sauer MJ, Foulkes JA, Worsfold A, \& Morris BA, 1986. Use of progesterone 11 glucuronide-alkaline phosphatase conjugate in a sensitive microtitre-plate enzymeimmunoassay of progesterone in milk and its application to pregnancy testing in dairy cattle. Journal of Reproduction \& Fertility 76: 375-391.

Sauerwein H, Heintges U, Hennie M, Selhorst T, \& Daxenberger A, 2004. Growth hormone induced alterations of leptin serum concentrationsin dairy cows as measured by a novel enzyme immunoassay. Livestock Production Science 87: 189-195.

Savino F \& Liguori SA, 2008. Update on breast milk hormones: Leptin, ghrelin and adiponectin. Clinical Nutrition 27: 42-47.

Scacchi M, Pincelli AI, \& Cavagnini F, 1999. Growth hormone in obesity. International Journal of Obesity 23: 260-271.

Scaramuzzi RJ, Brown HM, \& Dupont J, 2010. Nutritional and metabolic mechanisms in the ovary and their role in mediating the effects of diet on folliculogenesis: a perspective. Reprod. Dom. Anim. 45 (Suppl. 3): 32-41.

Schafer JL, 1997. Analysis of incomplete multivariate data. Chapman \& Hall, London, UK.

Schneider JE, 2004. Energy balance and reproduction. Physiology \& Behaviour 81: 289317. 
Schneider JE, Goldman MD, Tang S, Bean B, Ji H, \& Friedman MI, 1998. Leptin indirectly affects estrous cycles by increasing metabolic fuel oxidation. Horm Behav. 33(3): 217-228.

Schoenberg KM \& Overton TR, 2010. The changing roles of insulin during the transition period. Proceedings Cornell Nutrition Conference for Feed Manufacturers. $72^{\text {nd }}$ Meeting. pp 175-185.

Schoenberg KM, Smith KL, Ehrhardt RM, Boisclair YR, \& Overton TR, 2008. Effects of prepartum 2,4-thiazolidinedione on plasma leptin and insulin sensitivity in transition dairy cows. J Dairy Sci. 91(E-Suppl. 1): 62.

Selk GE, Wettemann RP, Lusby KS, Oltjen JW, Mobley SL, Rasby RJ, \& Garmendia JC, 1988. Relationships among weight change, body condition and reproductive performance of range beef cows. J Anim. Sci. 66: 3153-3159.

Selvaraju S, Agarwal SK, Karche SD, Srivastava SK, Majumdar AC, \& Shanker U, 2002. Fertility responses and hormonal profiles in repeat breeding cows treated with insulin. Anim. Reprod. Sci. 73: 141-149.

Selvin S, 2008. Survival analysis for epidemiologic and medical research. Cambridge University Press, UK.

Sesti G, Federici M, Hribal ML, Lauro D, Sbraccia P, \& Lauro R, 2001. Defects of the insulin receptor substrate (IRS) system in human metabolic disorders. The FASEB Journal 15: 2099-2111.

Sesti G, Sciacqua A, Cardellini M, Marini MA, Maio R, Vatrano M, Succurro E, Lauro R, Federici M, \& Perticone F, 2005. Plasma concentration of IGF-I is independently associated with insulin sensitivity in subjects with different degrees of glucose tolerance. Diabetes Care 28: 132-137.

Shetty S, Kusminski CM, \& Scherer PE, 2009. Adiponectin in health and disease: evaluation of adiponectin-targeted drug development strategies, Trends in Pharmacological Sciences 30(5): 234-239.

Shimabukuro M, Chinen I, Higa N, Takasu N, Yamakawa K, \& Ueda S, 2007. Effects of dietary composition on postprandial endothelial function and adiponectin concentrations in healthy humans: a crossover controlled study. Am. J Clin. Nutr. 86: 923-928.

Short RE, Bellows RA, Staigmiller RB, Berardinelli JG \& Custer EE, 1990. Physiological mechanisms controlling anestrus and infertility in postpartum beef cattle. $J$ Anim. Sci. 68: 799-816. 
Silha JV, Krsek M, Skrha JV, Sucharda P, Nyomba BLG, \& Murphy LJ, 2003. Plasma resistin, adiponectin and leptin levels in lean and obese subjects: correlations with insulin resistance. European Journal of Endocrinology 149: 331-335.

Sinclair KD, Kuran M, Gebbie FE, Webb R, \& McEvoy TG, 2000. Nitrogen metabolism and fertility in cattle II. Development of oocytes recovered from heifers offered diets differing in their rate of nitrogen release in the rumen. J Anim. Sci. 78: 26702680 .

Sinclair KD \& Garnsworthy PC, 2009. Fatty acids and fertility in dairy cows. In Recent advances in animal nutrition ( $e d \mathrm{~s}$ : Garnsworthy PC \& Wiseman J). Nottingham University Press, Nottingham, UK. pp 1-19.

Sinclair KD, 2010. Declining fertility, insulin resistance and fatty acid metabolism in dairy cows: Developmental consequences for the oocyte and pre-implantation embryo. Acta Scientiae Veterinariae 38(Supl 2): s545-s557

Sir-Petermann T, Echiburu B, Maliqueo MM, Crisosto N, Sanchez F, Hitschfeld C, Carcamo M, Amigo P, \& Perez-Bravo F, 2007. Serum adiponectin and lipid concentrations in pregnant women with polycystic ovary syndrome. Human Reproduction 22(7): 1830-1836.

Smith GH, 1971. Glucose metabolism in the ruminant. Proceedings of the Nutrition Society 30: 265-272.

Smith KL \& Overton TR, 2008. Research update: insulin resistance in transition cows. www.ansci.cornell.edu/pdfs/pd2008febp26.pdf. Accessed July 10, 2011.

Snijders SEM, Dillon PG, O'Farrell KJ, Diskin M, Wylie ARG, O'Callaghan D, Rath M, \& Boland MP, 2001. Genetic merit for milk production and reproductive success in dairy cows. Anim. Reprod. Sci. 65: 17-31.

Spicer LJ \& Echternkamp SE, 1995. The ovarian insulin-like growth factor system with an emphasis on domestic animals. Domest. Anim. Endocrinol. 12: 223-245.

Spicer LJ, Alpizar E, \& Echternkamp SE, 1993. Effects of insulin, Insulin-Like Growth Factor I, and gonadotropins on bovine granulosa cell proliferation, progesterone production, estradiol production, and(or) insulin-like growth factor i production in vitro. $J$ Anim. Sci. 71: 1232-1241.

Spicer LJ, Tucker WB, \& Adams GD, 1990. Insulin-like growth factor-I in dairy cows: Relationships among energy balance, body condition, ovarian activity, and estrous behaviour. J Dairy Sci. 73: 929-937. 
Spitzer JC, Morrison DG, Wettemann RP, \& Faulkner LC, 1995. Reproductive responses and calf birth and weaning weights as affected by body condition at parturition and postpartum weight gain in primiparous beef cows. J Anim. Sci. 73:1251-1257.

Spranger J, Kroke A, Mohlig M, Bergmann MM, Ristow M, Boeing H, \& Pfeiffer AF, 2003. Adiponectin and protection against type 2 diabetes mellitus. Lancet 361: 226-228.

Steen A, Grantor H \& Tureen PA, 1997. Glucose and insulin responses to glucagon injection in dairy cows with ketosis and fatty liver. Journal of Veterinary Medicine 44: 521-530.

Steinberg GR \& Kemp BE, 2007. Adiponectin: Starving for Attention. Cell Metab. 6: 23.

Stevenson JS, 2001. Reproductive management of dairy cows in high milk-producing herds. J Dairy Sci. 84 (E. Suppl.): E128-E143.

Steyn FJ, Boehme F, Vargas E, Wang K, Parkington HC, Rao JR, \& Chen C, 2009. Adiponectin regulate growth hormone secretion via adiponectin receptor mediated $\mathrm{Ca}_{2}^{+}$ signalling in rat somatotrophs in vitro. Journal of Neuroendocrinology 21: 698-704.

Stott AW, Veerkamp RF, \& Wassell TR, 1999. The economics of fertility in the dairy herd. Animal Science 68: 49-58.

Sutter F \& Beever DE, 2000. Energy and nitrogen metabolism in Holstein-Friesian cows during early lactation. Journal of Animal Science 70: 503-514.

Tabandeh MR, Hosseini A, Saeb M, Kafi M,\& Saeb S, 2010. Changes in the gene expression of adiponectin and adiponectin receptors (AdipoR1 and AdipoR2) in ovarian follicular cells of dairy cow at different stages of development. Theriogenology 73: 659669.

Taketani T, Yamagata Y, Takasaki A, Matsuoka A, Tamura H \& Sugino N, 2008. Effects of growth hormone and insulin-like growth factor 1 on progesterone production in human luteinized granulosa cells. Fertility \& Sterility 90(3): 744-748.

Tanaka T, Nagatani S, Bucholtz DC, Ohkura S, Tsukamura H, Maeda KI, \& Foster DL. Central action of insulin regulates pulsatile luteinizing hormone secretion in the diabetic sheep model. Biology of Reproduction 62: 1256-1261.

Taylor VJ, Beever DE, Bryant MJ, \& Wathes DC, 2003. Metabolic profiles and progesterone cycles in first lactation dairy cows. Theriogenology 59: 1661-1677.

Thomas C, Offer NW, Agnew RE, Cottrill BR, Givens DI, Keady TWJ, Mayne CS, Rymer C, Yan T, France J,\& Beever DE, 2004. Feed into milk - an applied feeding 
model coupled with a new system of feed characterization. In Recent advances in animal nutrition (eds.: Garnsworthy PC \& Wiseman J). Nottingham University Press, Nottingham, UK. pp 167-195.

Thorens B, 1996. Glucose transporters in the regulation of intestinal, renal, and liver glucose fluxes. Am. J Physiol. 270: G541-G553.

Thorn SR, Ehrhardt RA, Butler WR, Quirk SM, \& Boisclair YR, 2008. Insulin regulates hepatic leptin receptor expression in early lactating dairy cows. Am. J Physiol. Regul. Integr. Comp. Physiol. 295: 1455-1462.

Trenkle A, 1978. Relation of hormonal variations to nutritional studies and metabolism of ruminants. J Dairy Sci. 61: 281-293.

Treeck O, Lattrich C, Juhasz-Boess I, Buchholz S, Pfeiler G, \& Ortmann O, 2008. Adiponectin differentially affects gene expression in human mammary epithelial and breast cancer cells. British Journal of Cancer 99: 1246-1250.

Tschritter O, Fritsche A, Thamer C, Haap M, Shirkavand F, Rahe S, Staiger H, Maerker, E, Haring H, \& Stumvoll M, 2003. Plasma adiponectin concentrations predict insulin sensitivity of both glucose and lipid metabolism. Diabetes 52: 239-243.

USDA, 2011. Milk: Production per cow by year in the USA. http://www. nass.usda.gov/Charts_and_Maps/Milk_Production_and_Milk_Cows/cowrates.asp.

Accessed July 10, 2011.

Van Houtert MFJ, 1993. The production and metabolism of volatile fatty acids by ruminants fed roughages: A review. Animal Feed Science \& Technology 43: 189-225.

Vazquez-Vela MEF, Torres $\mathbf{N}, \&$ Tovar AR, 2008. White adipose tissue as endocrine organ and its role in obesity. Archives of Medical Research 39: 715-728.

Veerkamp RF, Beerda B, \& Van der Lende T, 2003a. Effects of genetic selection for milk yield on energy balance, levels of hormones, and metabolites in lactating cattle, and possible links to reduced fertility. Livestock Production Science 83: 257-275.

Veerkamp RF, Koenen EPC, \& De Jong G, 2001. Genetic correlations among body condition score, yield, and fertility in first-parity cows estimated by random regression models. J Dairy Sci. 84: 2327-2335.

Veerkamp RF, Oldenbroek JK, Van Der Gaast HJ, \& Van Der Werf JHJ, 2000. Genetic correlation between days until start of luteal activity and milk yield, energy balance, and live weights. J Dairy Sci. 83: 577-583. 
Velazquez MA, Spicer LJ, \& Wathes DC, 2008. The role of endocrine insulin-like growth factor-I (IGF-I) in female bovine reproduction. Domestic Animal Endocrinology 35: $325-342$.

Vernon RG \& Pond CM, 1997. Adaptations of maternal adipose tissue to lactation. Journal of Mammary Gland Biology \& Neoplasia, 2(3): 131-141.

Vernon RG, 2005. Lipid metabolism during lactation: a review of adipose tissue-liver interactions and the development of fatty liver. Journal of Dairy Research 72: 460-469.

Vicini JL, Buonomo FC, Veenhuizen JL, Miller MA, Clemmons DR, \& Collier RJ, 1991. Nutrient balance and stage of lactation affect responses of insulin, insulin-like growth factors I and II, and insulin-like growth factor-binding protein 2 to somatotropin administration in dairy cows. $J$ Nutr. 121: 1656-1664.

Villa-Godoy A, Hughes TL, Emery RS, Chapin LT \& Fogwell RL, 1988. Association between energy balance and luteal function in lactating dairy cows. J Dairy Sci. 71: 10631072.

Villa-Godoy A, Hughes TL, Emery RS, Stanisiewski EP, \& Fogwell RL, 1990. Influence of energy balance and body condition on estrus and estrous cycles in Holstein heifers. $J$ Dairy Sci. 73: 2759-2765.

Wade GN \& Schneider JE, 1992. Metabolic fuels and reproduction in female mammals. Neuroscience \& Biobehavioral Reviews 16: 235-272.

Wagner JD, Zhang L, Shadoan MK, Kavanagh K, Chen H, Tresnasarid K, Kaplan JR, \& Adams MR, 2008. Effects of soy protein and isoflavones on insulin resistance and adiponectin in male monkeys. Metabolism Clinical \& Experimental 57(Suppl 1): S24-S31.

Wang $\mathbf{Z} \&$ Goonewardene LA, 2004. The use of MIXED models in the analysis of animal experiments with repeated measures data. Can. J. Anim. Sci 84: 1-11.

Waltner SS, McNamara JP, \& Hillers JK, 1993. Relationships of body condition score to production variables in high producing Holstein dairy cattle. J Dairy Sci. 76: 34103419.

Wathes DC, 2008. Metabolic effects on the reproductive tract environment and conception rates in the dairy cow. Havemeyer Found Monogr Ser. 21: 15-19.

Wathes DC, Cheng Z, Bourne N, Taylor VJ, Coffey MP, \& Brotherstone S, 2007. Differences between primiparous and multiparous dairy cows in the inter-relationships between metabolic traits, milk yield and body condition score in the periparturient period. Domestic Animal Endocrinology 33: 203-225. 
Wayman JC, 2003. Multiple imputation for missing data: What is it and how can I use it? Paper presented at the 2003 Annual Meeting of the American Educational Research Association, Chicago, IL, USA. Download from http://www.csos.jhu.edu/ contact/staff/jwayman_pub/wayman_multimp_aera2003.pdf. Accessed August 11, 2011.

Webb R, Garnsworthy PC, Campbell BK \& Hunter MG 2007. Intra-ovarian regulation of follicular development and oocyte competence in farm animals. Theriogenology 68S: S22-S29.

Webb R, Garnsworthy PC, Gong JG and Armstrong DG, 2004. Control of follicular growth: local interactions and nutritional influences. J Anim. Sci. 82: 63-74.

Werner C \& Schermelleh-Engel K, 2009. Structural equation modeling: advantages, challenges, and problems. http://user.uni-frankfurt.de/ cswerner/sem/. Accessed July 10, 2011.

Westwood, CT, Lean IJ, Garvin JK, \& Wynn P, 2000. Effects of genetic merit and varying dietary protein degradability on lactating dairy cows. J Dairy Sci. 83: 2926-2940.

Weyer C, Funahashi T, Tanaka S, Hotta K, Matsuzawa Y, Pratley RE, \& Tataranni PA, 2001. Hypoadiponectinemia in obesity and type 2 diabetes: Close association with insulin resistance and hyperinsulinemia. J. Clin. Endocrinol. Metab. 86: 1930-1935.

Weyermann M, Beermann C, Brenner H, \& Rothenbacher D, 2006. Adiponectin and leptin in maternal serum, cord blood, and breast milk. Clinical Chemistry 52: 11-19.

Williams CJ, Fargnoli JL, Hwang JJ, Van Dam RM, Blackburn GL, Hu FB, \& CS Mantzoros, 2008. Coffee consumption is associated with higher plasma adiponectin concentrations in women with or without type 2 diabetes: a prospective cohort study. Diabetes Care 31(3): 504-507.

Williams GL, Amstalden M, Garcia MR, Stanko RL, Nizielski SE, Morrison CD, \& Keisler DH, 2002. Leptin and its role in the central regulation of reproduction in cattle. Domestic Animal Endocrinology 23: 339-349.

Williams T, Berelowitz M, Joffe SN, Thorner MO, Rivier J, Vale W, \& Frohman LA, 1984. Impaired growth hormone responses to growth hormone-releasing factor in obesity a pituitary defect reversed with weight reduction. N. Engl. J. Med. 311: 1403-1407.

Windig JJ, Beerda B, \& Veerkamp RF, 2008. Relationship between milk progesterone profiles and genetic merit for milk production, milking frequency, and feeding regimen in dairy cattle. J Dairy Sci. 91: 2874-2884.

Woodward M, 1999. Epidemiology, study design, and data analysis. In Texts in statistical science series (eds: Chatfield C \& Zidek JV). Chapman \& Hall/CRC, London, UK. 
Wright IA, Rhind SM, Russel AJF, Whyte TK, McBean AJ, \& McMillen SR, 1987. Effects of body condition, food intake and temporary calf separation on the duration of the post-partum anoestrous period and associated LH, FSH, and prolactin concentrations in beef cows. Anim. Prod. 45: 395-402.

Xu A, Wang Y, \& Lam KSL, 2007. Adiponectin. In adipose tissue and adipokines in health and disease (eds: Fantuzzi G \& Mazzone T). Humana Press Inc., Totowa, New Jersey, USA. pp 47-59.

Yamada K, Nakao T, \& Isobe N, 2003. Effects of body condition score in cows peripartum on the onset of postpartum ovarian cyclicity and conception rates after ovulation synchronization/fixed-time artificial insemination. J Reprod. Dev. 49: 381-388.

Yamauchi T, Kamon J, Ito Y, Tsuchida A, Yokomizok T, Kita S, Sugiyama T, Miyagishi M, Hara K, Tsunoda M, Murakami K, Ohteki T, Uchida S, Takekawa S, Waki H, Tsuno NH, Shibata Y, Terauchi Y, Froguel P, Tobe K, Koyasu S, Taira K, Kitamura T, Shimizuk T, Nagai R, \& Kadowaki T, 2003. Cloning of adiponectin receptors that mediate antidiabetic metabolic effects. Nature 423: 762-769.

Yamauchi T, Kamon J, Minokoshi Y, Ito Y, Waki H, Uchida S, Yamashita S, Noda M, Kita S, Ueki K, Eto K, Akanuma Y, Froguel P, Foufelle F, Ferre P, Carling D, Kimura S, Nagai R, Kahn BB \& Kadowaki T, 2002. Adiponectin stimulates glucose utilization and fatty-acid oxidation by activating AMP-activated protein kinase. Nature Medicine 8 (11): 1288-1295.

Yamauchi T, Kamon J, Waki H, Terauchi Y, Kubota N, Hara K, Mori Y,Ide T, Murakami K, Tsuboyama-Kasaoka N, Ezaki O, Akanuma Y, Gavrilova O, Vinson C, Reitman MI, Kagechika H, Shudo K, Yoda M, Nakano Y, Tobe K, Nagai R, Kimura S, Tomita M, Froguel P \& Kadowaki T, 2001. The fat-derived hormone adiponectin reverses insulin resistance associated with both lipoatrophy and obesity. Nature Medicine 7 (8): 941-946.

Yildiz BO \& Haznedaroglu IC, 2006. Rethinking leptin and insulin action: Therapeutic opportunities for diabetes, The International Journal of Biochemistry and Cell Biology 38: 820-830.

Yoon YS, Ryu D, Lee MW, Hong S, \& Koo SH, 2009. Adiponectin and thiazolidinedione targets CRTC2 to regulate hepatic gluconeogenesis. Experimental \& Molecular Medicine 41(8): 577-583.

Youngren JF, 2007. Regulation of insulin receptor function. Cell. Mol. Life Sci. 64: 873891. 
Zavalza-Gomez AB, Anaya-Prado R, Rincon-Sanchez AR, \& Mora-Martınez JM 2008. Adipokines and insulin resistance during pregnancy. Diabetes Research \& Clinical Practice 8 (0): 8-15.

Zhao FQ \& Keating AF, 2007. Expression and regulation of glucose transporters in the bovine mammary gland. $J$ Dairy Sci. 90(E. Suppl.): E76-E86.

Zieba DA, Amstalden M, \& Williams GL, 2005. Regulatory roles of leptin in reproduction and metabolism: A comparative review. Domestic Animal Endocrinology 29: 166-185.

Zieba DA, Amstalden M, Morton S, Gallino JL, Edwards JF, Harms PG, \& Williams GL, 2003. Effects of leptin on basal and GHRH-stimulated GH secretion from the bovine adenohypophysis are dependent upon nutritional status. Journal of Endocrinology 178: 8389.

Zorn JW, 2000. Comparing GEE and robust standard errors, with an application to judicial voting. Paper prepared for presentation at the Annual Meeting of the Southern Political Science Association, November 9-11, 200, Atlanta, GA. Download from http://polmeth.wustl.edu/media/Paper/zorn00.pdf. Accessed August 11, 2011.

Zumbach MS, Boehme MWJ, Wahl P, Stremmel W, Ziegler R, \& Nawroth PP, 1997. Tumor necrosis factor increases serum leptin levels in humans. J Clin. Endocrinol. Metab. 82: 4080-4082.

Zou KH, OMalley AJ \& Mauri L, 2011. Receiver-operating characteristic analysis for evaluating diagnostic tests and predictive models. Circulation 115: 654-657. 